\title{
The Syndrome of \\ Transient Epileptic Amnesia
}

\author{
Christopher R. Butler
}

MA (Cantab) MB ChB (Edin) MRCP (UK)

Thesis submitted in fulfilment of the requirements for the degree of $\mathrm{PhD}$ The University of Edinburgh.

Edinburgh

2008 



\section{Declaration}

I declare that this thesis was composed by me and that the work contained therein is my own, except where explicitly stated otherwise in the text.

The work within this thesis has not been submitted for any other degree or professional qualification.

Christopher Butler

2008 


\section{Acknowledgements}

I am deeply grateful to the patients who participated in this study and to their families for their interest, enthusiasm, hospitality and patience during countless hours of interviewing and neuropsychological testing.

Many people assisted with the development and execution of the TIME Project. Colleagues in neurology and psychiatry from around the United Kingdom referred patients. Staff at the Wellcome Trust Clinical Research Facility in Edinburgh and at the Medical Research Council Cognition and Brain Sciences Unit in Cambridge helped arrange interviews with participants. Rosemary Anderson provided cheery and dependable secretarial support. Lorna Torrens trained me in the techniques of neuropsychological assessment. Roger Cull helped analyse electroencephalographic data. Ian Deary granted access to vascular risk factor data from the Lothian Birth Cohort 1936 Study. Staff at the Brain Imaging Research Centre in Edinburgh and at the MRIS unit, Addenbrooke's Hospital, Cambridge performed the neuroimaging. Tom MacGillivray and Calum Gray lent their technical expertise to the processing and analysis of imaging data. Marilu Gorno-Tempini, Peter Nestor and Julio AcostaCabronero gave advice on and assistance with voxel-based morphometry.

I had great fun supervising two excellent fourth-year medical students during their Special Study Modules: Justin Penge neatly summarised a mountain of data on cerebrovascular disease risk factors and Amit Bhaduri demonstrated remarkable stamina and proficiency in measuring hippocampal and amygdalar volumes.

Particular thanks go to those who collaborated most closely with the TIME Project. Joanna Wardlaw oversaw the neuroimaging and donated a lot of her time to reviewing the MRI scans. I had many enlightening discussions with Narinder Kapur and Kim Graham, whose neuropsychological experience and scientific acumen were invaluable. John Hodges, my assistant supervisor, provided much wise counsel. 
My greatest debt of gratitude is to Adam Zeman, who has supported, inspired and guided me throughout this project. He has given generously of his time and friendship and has shown me that being able to combine lofty neuroscience with down-to-earth patient care is among the greatest of skills and privileges.

Finally, I thank my wife Sofie for her energy, patience and love and my children, Amaya, Aidan, Lukas and Charlotte, all of whom are younger than this study and will be glad to see the back of it! This thesis is dedicated to them.

The work in this thesis was generously funded by a research fellowship from the Patrick Berthoud Charitable Foundation. 


\section{Contents}

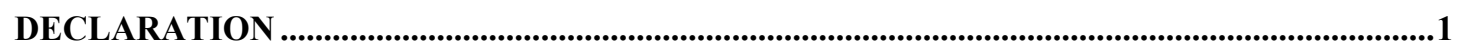

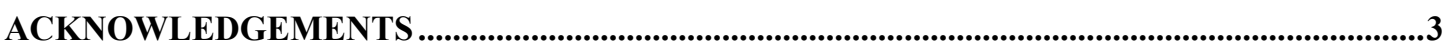

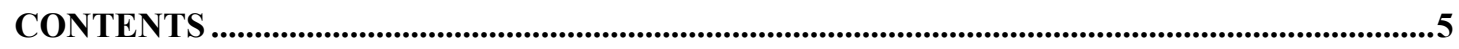

ABSTRACT ........................................................................................................................................13

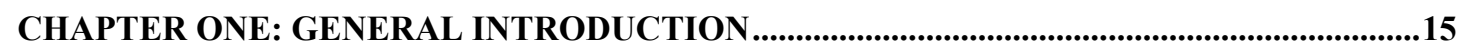

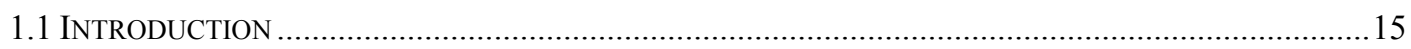

1.2 TRANSIENT EPILEPTIC AMNESIA: HISTORICAL DEVELOPMENT OF THE CONCEPT ...............................16

1.2.1 The case of $\mathrm{Dr} Z$

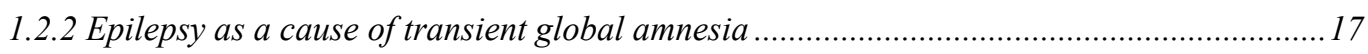

1.2.3 Early characterisation of TEA as a distinct neurological entity .......................................19

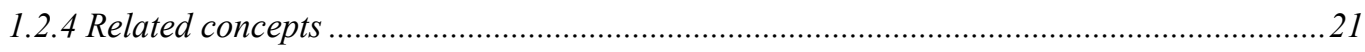

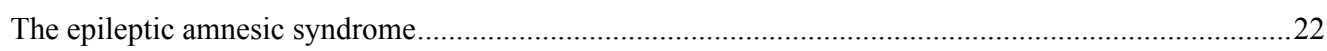

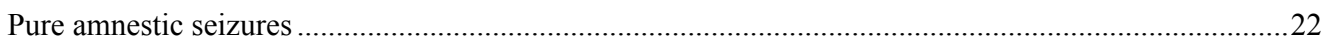

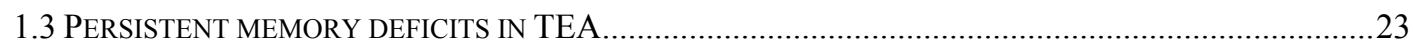

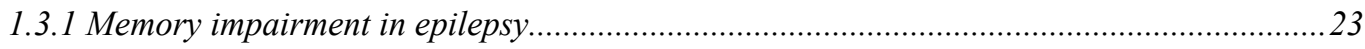

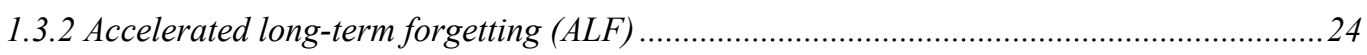

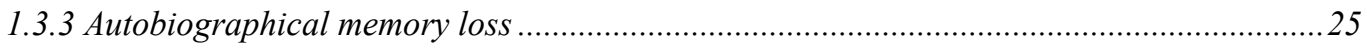

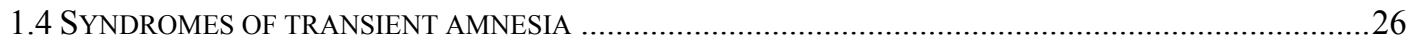

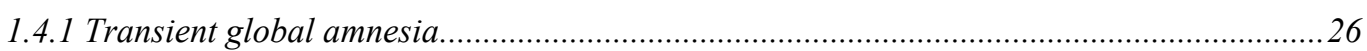

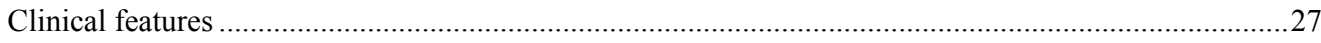

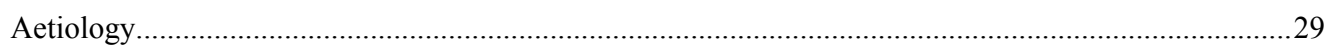

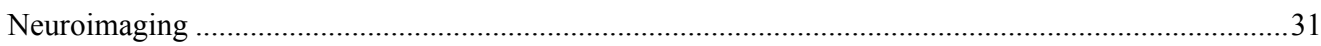

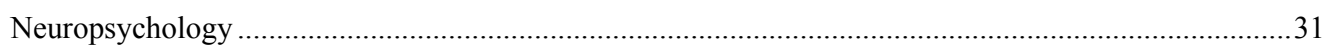

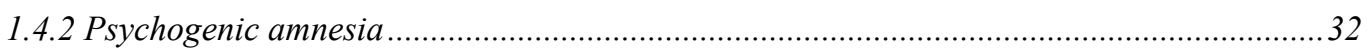

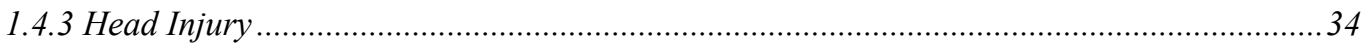

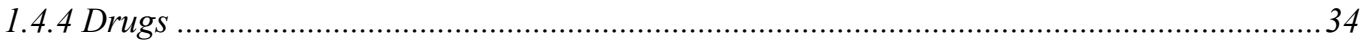

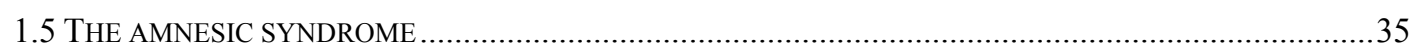

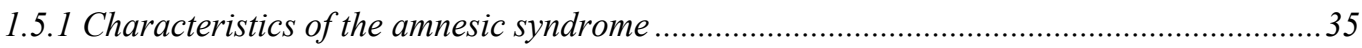

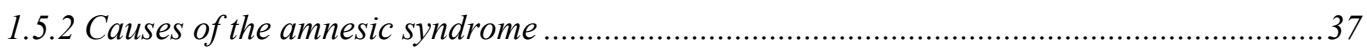

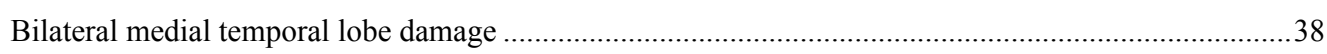

Korsakoff's syndrome

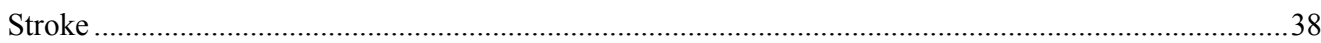

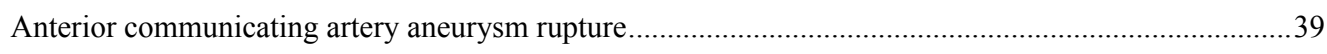

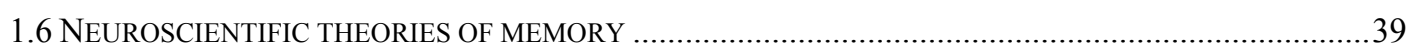

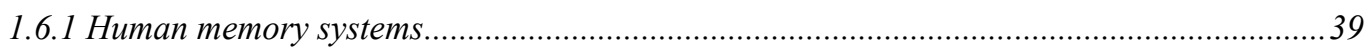

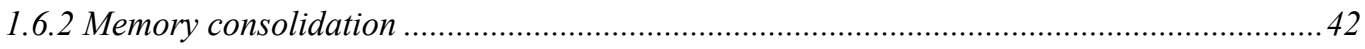

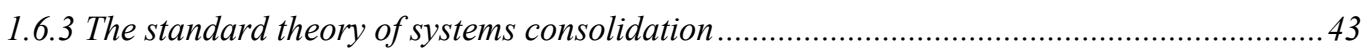




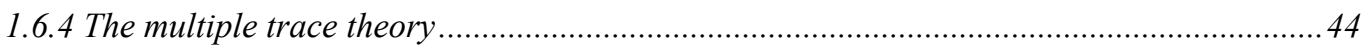

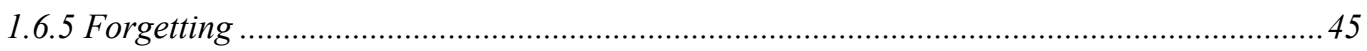

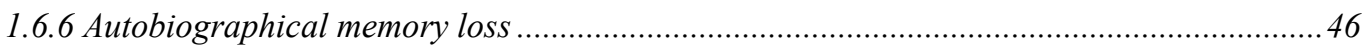

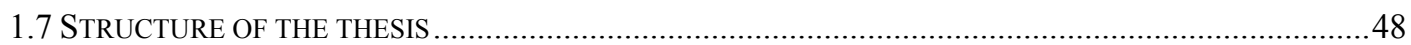

\section{CHAPTER TWO: THE CLINICAL CHARACTERISTICS OF TRANSIENT EPILEPTIC}

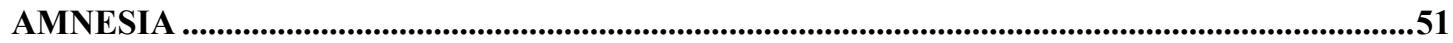

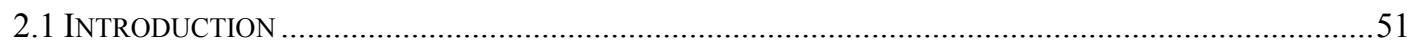

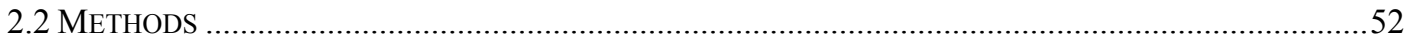

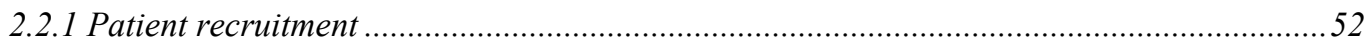

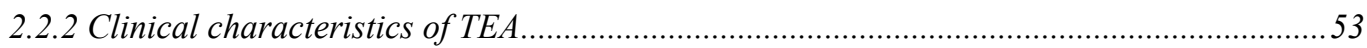

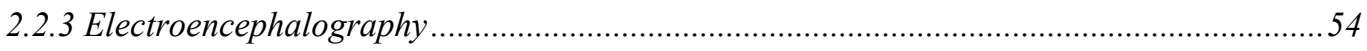

2.2.4 Cerebrovascular disease risk factors and TEA ............................................................... 54

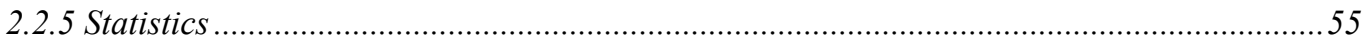

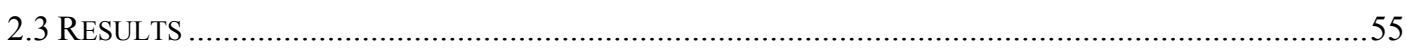

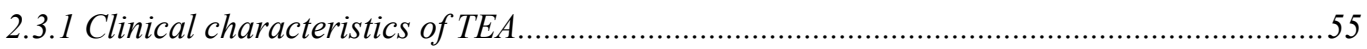

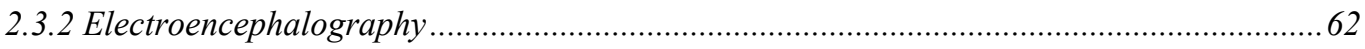

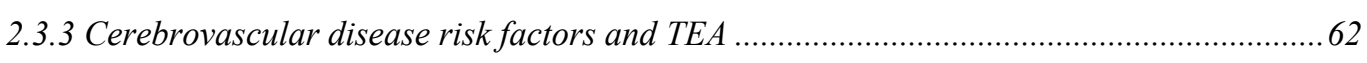

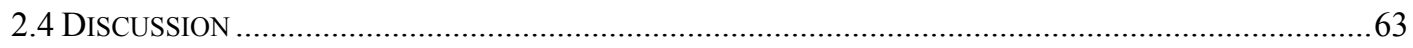

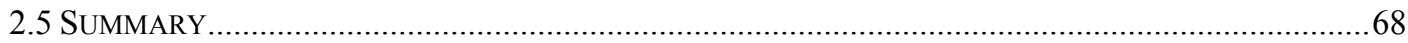

\section{CHAPTER THREE: A COMPREHENSIVE REVIEW OF TRANSIENT EPILEPTIC}

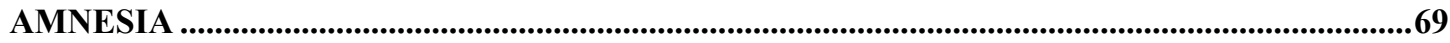

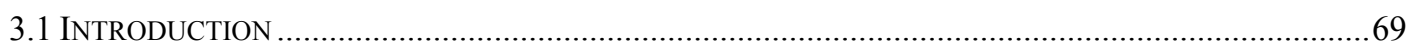

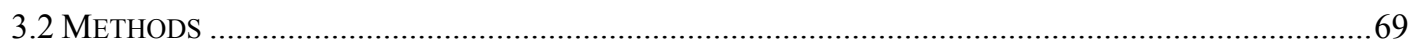

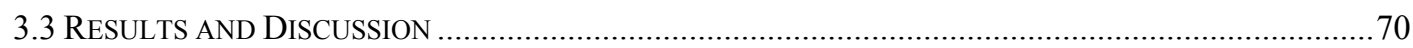

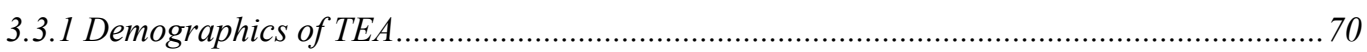

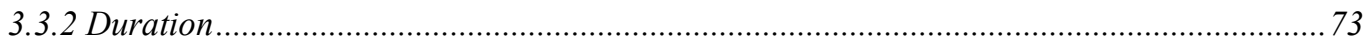

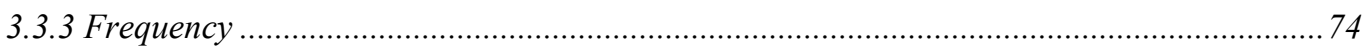

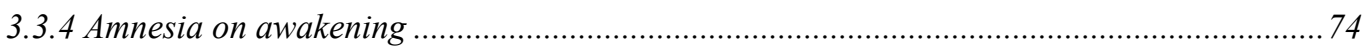

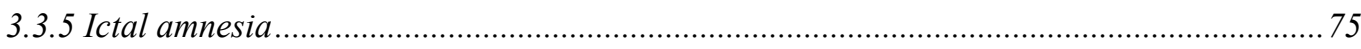

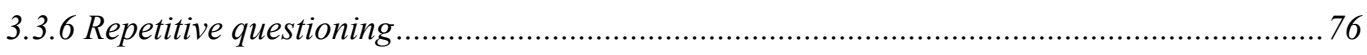

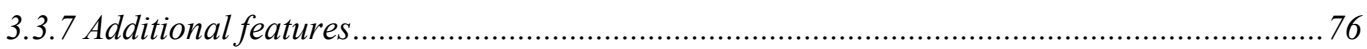

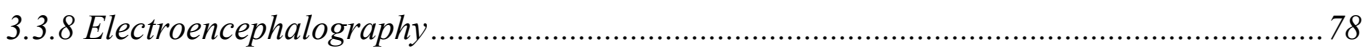

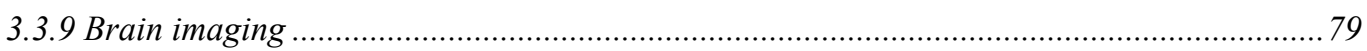

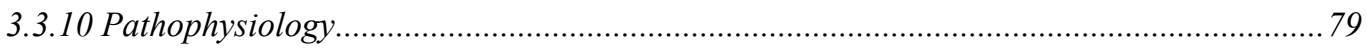

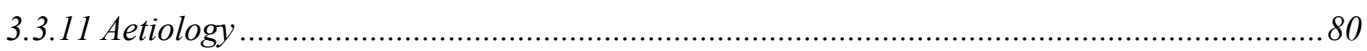

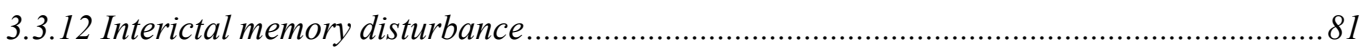

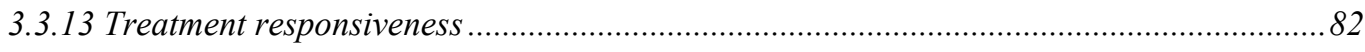

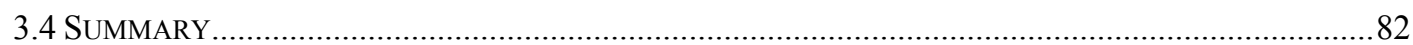




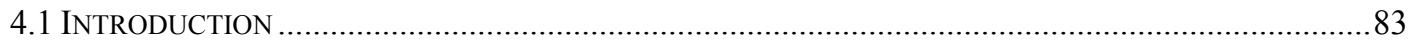

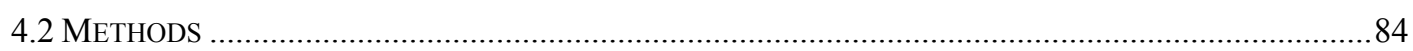

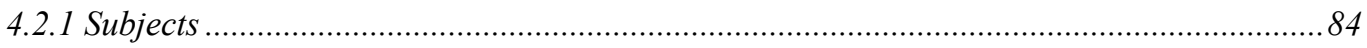

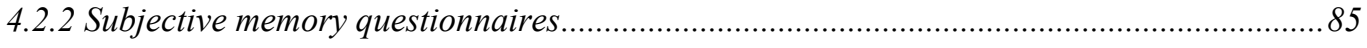

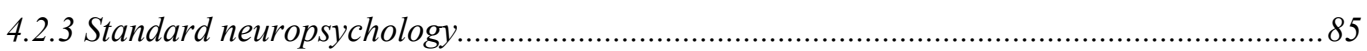

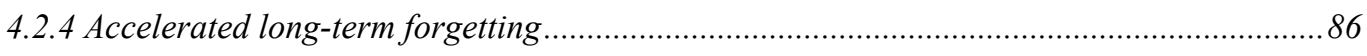

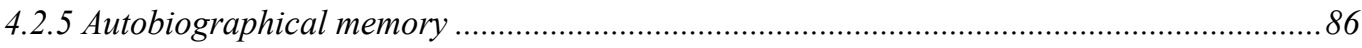

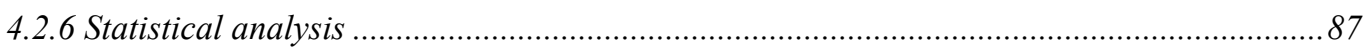

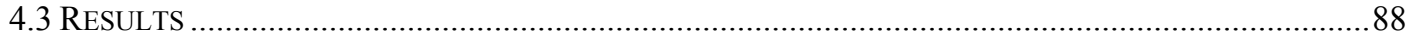

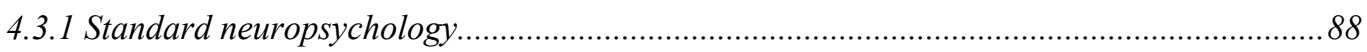

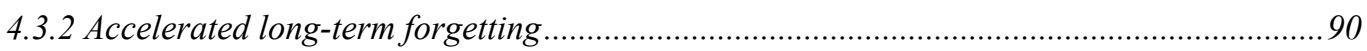

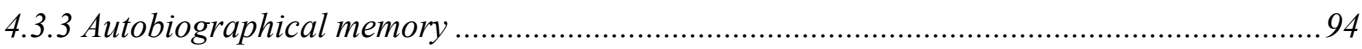

4.3.4 Relationship between ALF and autobiographical memory ...............................................95

4.3.5 Subjective memory scores and their relation to objective measures ..................................95

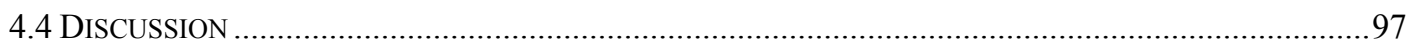

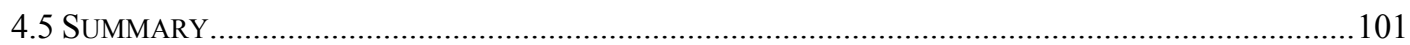

\section{CHAPTER FIVE: A COMPREHENSIVE REVIEW OF ACCELERATED LONG-TERM} FORGETTING AND REMOTE MEMORY IMPAIRMENT IN EPILEPSY .............................103

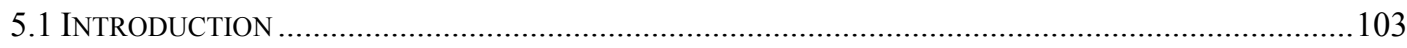

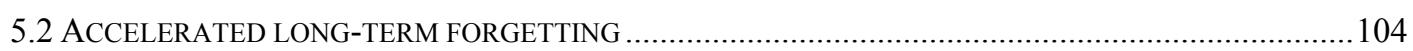

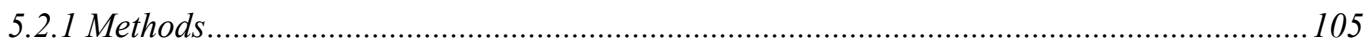

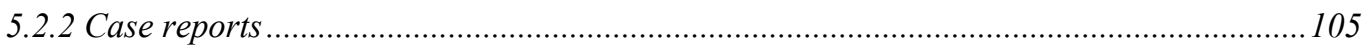

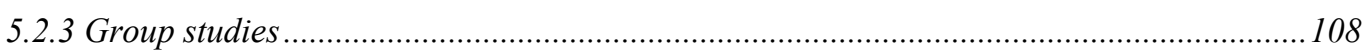

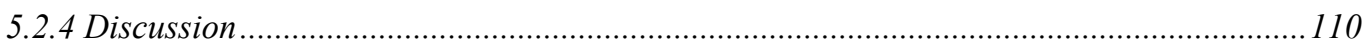

5.2.4.1 Does ALF occur in epilepsy? If so, for which types of material and is there a laterality effect? 110

5.2.4.2 Methodological issues in the assessment of ALF ..................................................................... 111

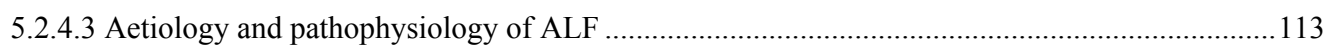

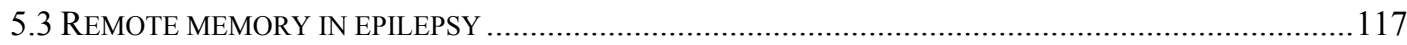

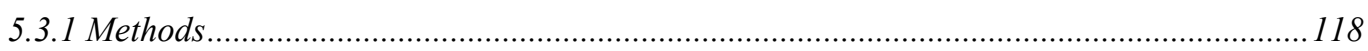

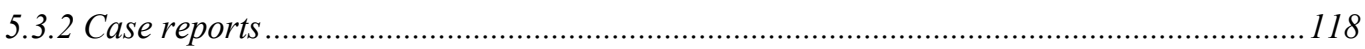

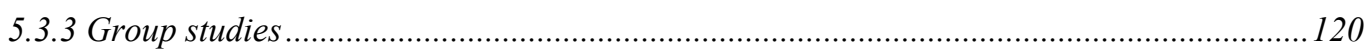

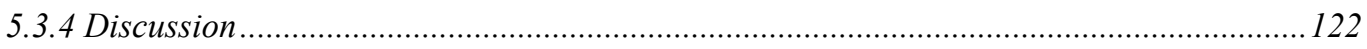

5.3.4.1 Is remote memory impaired in temporal lobe epilepsy? If so, for which types of material and is

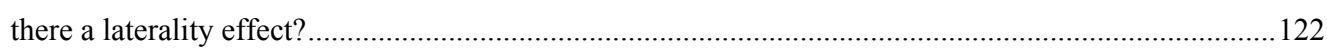

5.3.4.2 Methodological issues in the assessment of remote memory .................................................... 123

5.3.4.3 The nature of the deficit: the relationship between anterograde and remote memory loss and the temporal gradient of remote memory .

5.3.4.4 Aetiology and pathophysiology of RMI in epilepsy. 


\section{CHAPTER SIX: THE RADIOLOGICAL CHARACTERISTICS OF TRANSIENT EPILEPTIC}

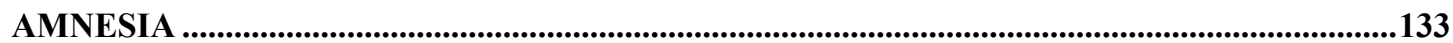

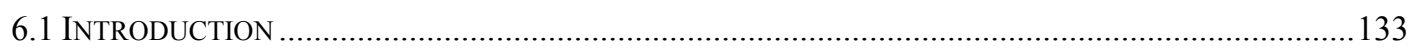

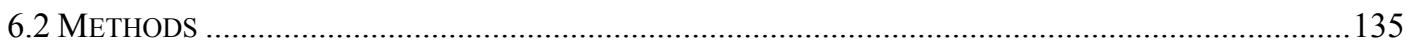

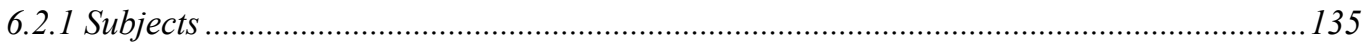

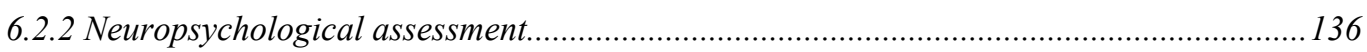

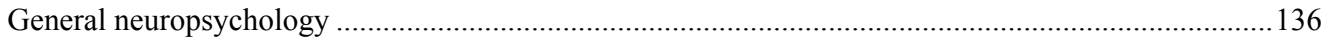

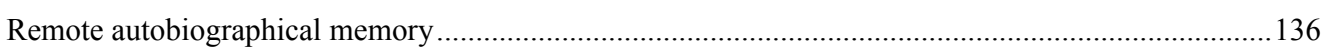

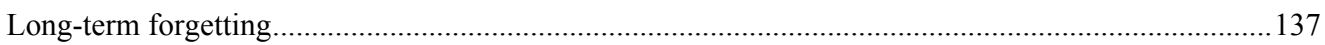

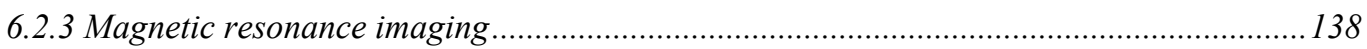

6.2.4 Neuroimaging markers of cerebrovascular disease .................................................. 139

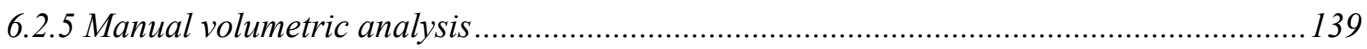

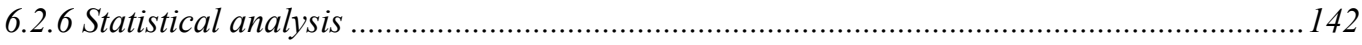

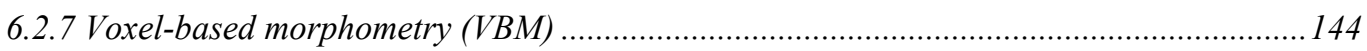

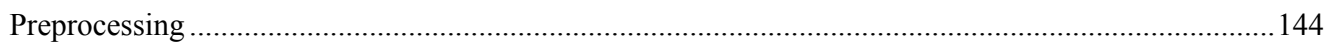

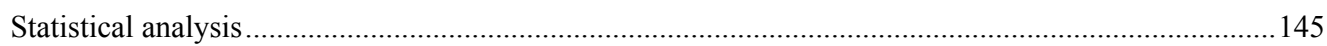

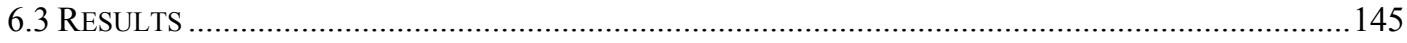

6.3.1 Clinical neuroradiological assessment .................................................................... 145

6.3.2 Neuroimaging markers of cerebrovascular disease ................................................... 146

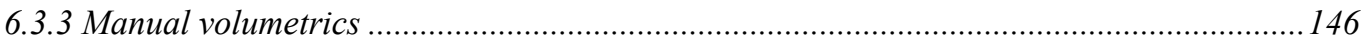

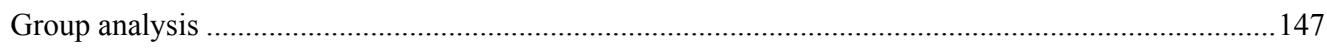

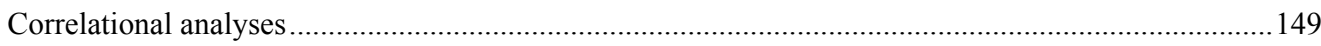

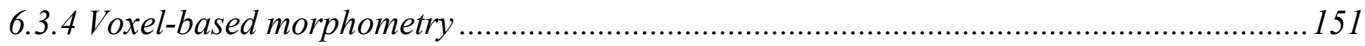

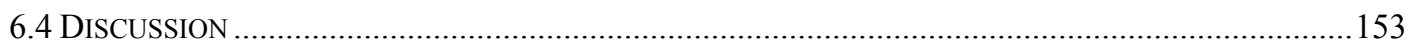

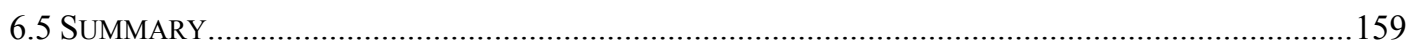

\section{CHAPTER SEVEN: TRANSIENT EPILEPTIC AMNESIA: A DETAILED CASE REPORT}

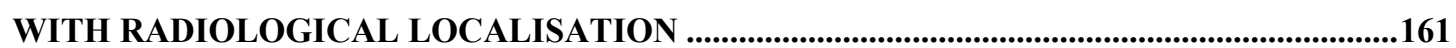

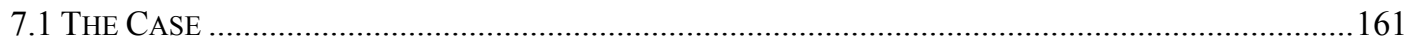

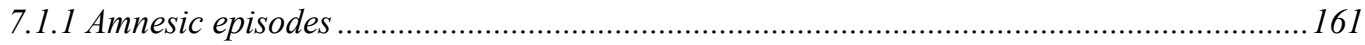

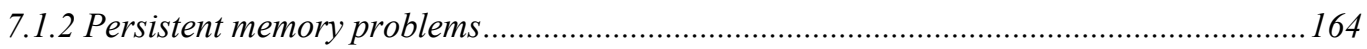

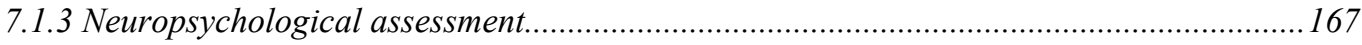

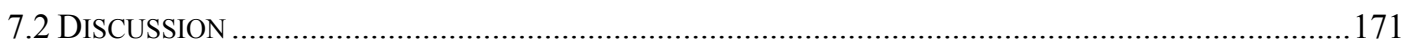

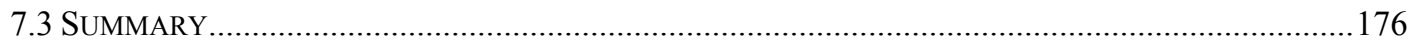

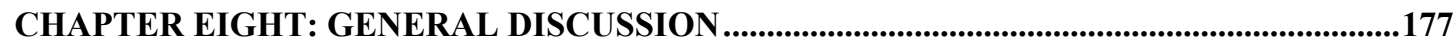

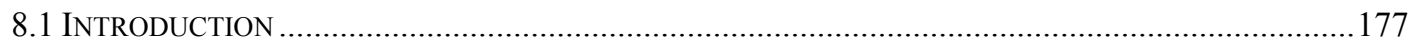

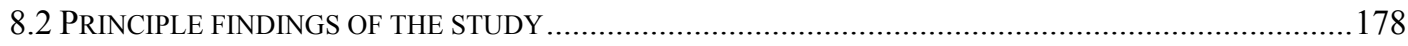

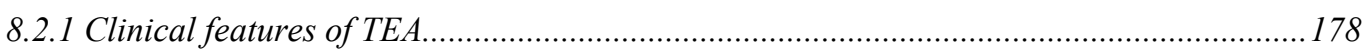

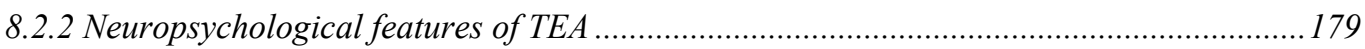




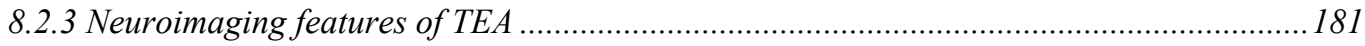

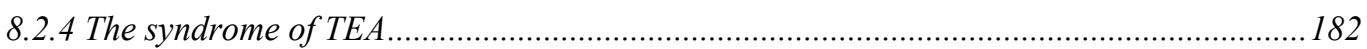

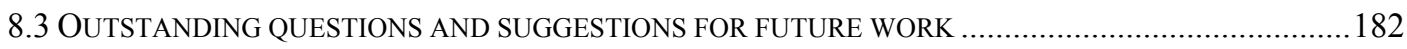

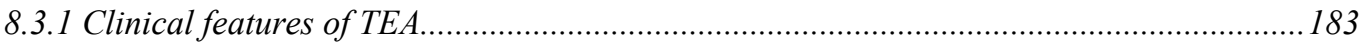

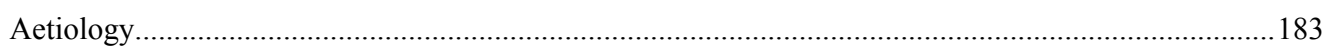

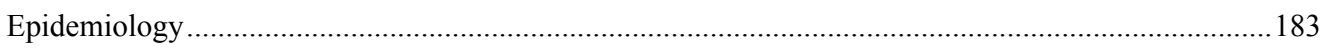

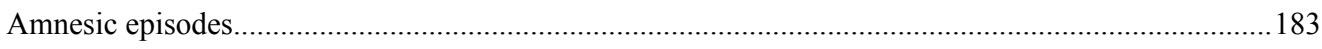

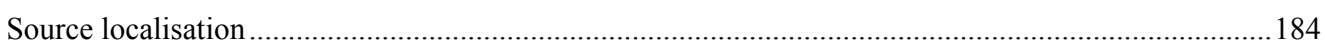

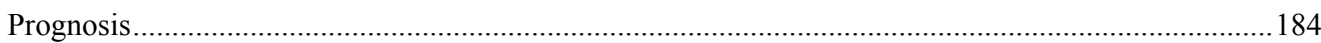

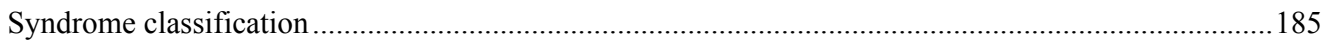

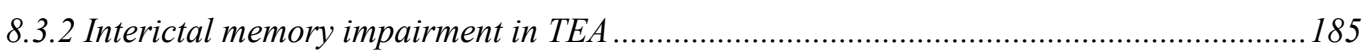

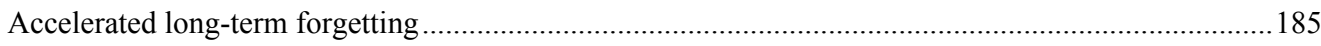

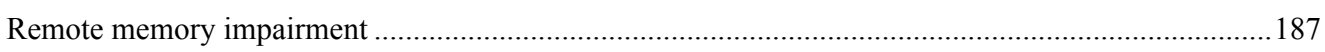

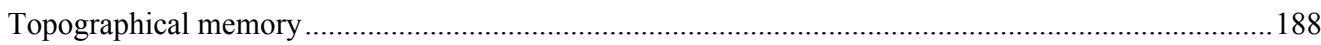

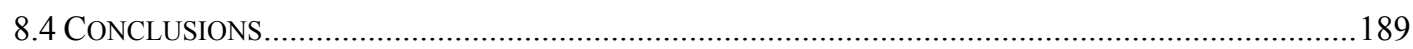

REFERENCES

APPENDIX A: TEA PATIENT HISTORY PRO FORMA .........................................................223

APPENDIX B: MRI SCAN VISUAL ASSESSMENT PRO FORM ..............................................227

APPENDIX C: ILLUSTRATIVE CASE REPORTS OF TRANSIENT EPILEPTIC

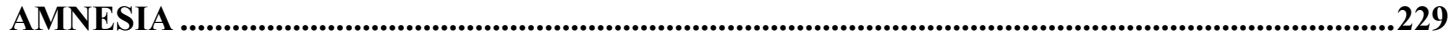

APPENDIX D: ILLUSTRATIVE CASES OF RECURRENT TRANSIENT AMNESIA

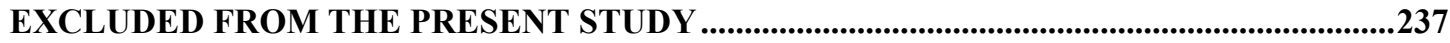

APPENDIX E: EVERYDAY MEMORY QUESTIONNAIRE...............................................239

APPENDIX F: VERY LONG TERM MEMORY QUESTIONNAIRE .......................................241

APPENDIX G: LONG-TERM FORGETTING TASKS..........................................................245

APPENDIX H: MODIFIED AUTOBIOGRAPHICAL MEMORY INTERVIEW (MAMI) ......253

APPENDIX I: TIME MRI SCANNING PROTOCOL ....................................................................257

PUBLICATIONS, PRESENTATIONS AND PRIZES RESULTING FROM THE WORK

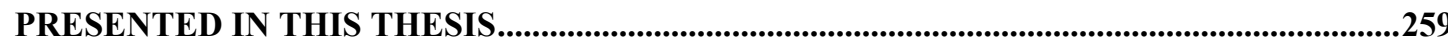


"No better neurologic work can be done than the precise investigation of epileptic paroxysms."

(Hughlings-Jackson, 1888, p188)

"A proper understanding of the amnesia accompanying epileptic attacks would contribute much to an understanding of the neurophysiological mechanisms of formation of memory patterns and of recall."

(Nielsen, 1958, p62) 


\begin{abstract}
Transient epileptic amnesia (TEA) is a form of epilepsy of which the principle manifestation is recurrent, transient episodes of isolated memory loss. Although the phenomenon has been recognised for over a century, it is scantily documented in the medical literature and is often misdiagnosed by clinicians. Recent work has highlighted a number of apparently consistent clinical features among the published cases. However, to date there has been no large, systematic study of the condition. The aim of the work reported in this thesis was to investigate a substantial number of prospectively recruited patients with TEA, and thus be able to provide a detailed and authoritative description of its clinical, neuropsychological and radiological characteristics.
\end{abstract}

Fifty patients with TEA were recruited from around the United Kingdom using established diagnostic criteria, together with a group of matched healthy control subjects. Participants underwent a clinical interview, comprehensive neuropsychological testing and structural magnetic resonance imaging of the brain.

The study demonstrated the following features. TEA typically begins in later life. The amnesic episodes are frequent, brief and often occur upon waking. They are characterised by a mixed anterograde and retrograde amnesia, the anterograde component of which is often incomplete. Attacks are commonly associated with olfactory hallucinations. They respond well to anticonvulsant medication. Nevertheless, many patients complain of persistent difficulties with memory. Despite generally performing well on standard tests of anterograde memory, many patients show i) accelerated forgetting of new information over a three-week delay and ii) temporally extensive deficits in autobiographical memory. TEA is associated with subtle medial temporal lobe atrophy on magnetic resonance imaging. This atrophy correlates with performance on standard memory tests, but not with long-term forgetting rates or autobiographical memory deficits.

It is proposed that TEA is a distinctive syndrome of epilepsy, typically misdiagnosed at presentation, caused by medial temporal seizure activity and associated with accelerated long-term forgetting and autobiographical memory loss. These unusual forms of memory impairment have been documented in other forms of epilepsy. They pose challenges to current models of memory. The syndrome of TEA is therefore both clinically and theoretically important. 


\section{CHAPTER ONE}

\section{General Introduction}

\subsection{Introduction}

The work reported in this thesis is an investigation of the clinical, neuropsychological and radiological characteristics of transient epileptic amnesia (TEA) - a form of epilepsy of which the principle manifestation is transient, isolated impairment of memory. The phenomenon has been recognised for over a century, but the relevant literature is sparse and is dominated by reports of individual patients and small case series. Recent work has highlighted a striking consistency in the conjunction of clinical features exhibited by these cases: the amnesic episodes usually begin in later life, often occur upon waking, are of relatively brief duration and respond well to anticonvulsant medication. In addition, many patients complain of unusual, persistent memory deficits. However, TEA remains largely unrecognised as a distinct neurological entity, and gains no mention in recent comprehensive textbooks of neurology (e.g. Bradley et al., 2008) or epilepsy (e.g. Engel et al., 2007).

The principle aim of the present work was to study a large, prospectively recruited cohort of patients with amnesic episodes due to seizures and thus be able to provide, for the first time, a detailed, authoritative description of the condition. A further aim was to investigate the unusual, persistent memory deficits that patients describe: to document their existence and explore their neuroanatomical basis.

In this introductory chapter, I first review the evolution of the concept of TEA and describe the principle clinical features as currently understood. I then discuss the interictal memory problems associated with TEA in the context of memory impairment in other forms of epilepsy. In section 1.4, I review other syndromes of transient amnesia and, in section 1.5, the amnesic syndrome more generally. Finally, in section 1.6 I provide an overview of relevant areas in the cognitive neuroscience of memory - in particular the concepts of memory consolidation and focal retrograde amnesia. 


\subsection{Transient epileptic amnesia: historical development of the concept}

\subsubsection{The case of $\operatorname{Dr} Z$}

In 1888, the renowned British neurologist John Hughlings-Jackson (Figure 1.1a) described the case of Dr Z, a medical practitioner who suffered from an unusual variety of epilepsy (Hughlings-Jackson, 1888). During seizures, Z retained consciousness and was able to engage in complex, purposeful behaviour for which he was later amnesic. Several such attacks are reported in Z's own words. One is particularly striking:

"I was attending a young patient whom his mother had brought me with some history of lung symptoms. I had wished to examine the chest, and asked him to undress on a couch. I thought he looked ill, but have no recollection of any intention to recommend him to take to his bed at once, or of any diagnosis. Whilst he was undressing I felt the onset of a petit mal. I remember taking out my stethoscope and turning away a little to avoid conversation. The next thing I recollect is that I was sitting at a writing-table in the same room, speaking to another person, and as my consciousness became more complete, recollected my patient, but saw that he was not in the room. I was interested to ascertain what had happened, and had an opportunity an hour later of seeing him in bed, with the note of a diagnosis I had made of 'pneumonia of the left base'. I gathered indirectly from conversation that I had made a physical examination, written these words, and advised him to take to bed at once. I reexamined him with some curiosity, and found that my conscious diagnosis was the same as my unconscious, - or perhaps I should say, unremembered diagnosis had been. I was a good deal surprised, but not so unpleasantly as I should have thought probable.” (Hughlings-Jackson, 1888)

On other occasions, Z's "petits maux" were accompanied by "a feeling of Recollection, i.e. of realising that what is occupying the attention is what has occupied it before" - a misplaced sense of familiarity that would today be called déjà $\mathrm{vu}$ - and a "smacking of the tongue like a tasting movement". Hughlings-Jackson referred to this variety of epilepsy as the "dreamy state", and adds hallucinations of smell and taste to the list of typical features. 
About 10 years later, Z died from an overdose of chloral hydrate. Autopsy of the brain revealed a "small cavity apparently due to softening" in the uncus of the left temporal lobe (Hughlings-Jackson and Colman, 1898) (see Figure 1.1b). Similar observations were made in a number of other patients, leading Hughlings-Jackson to refer to the “uncinate group of seizures" (Hughlings-Jackson and Stewart, 1899).

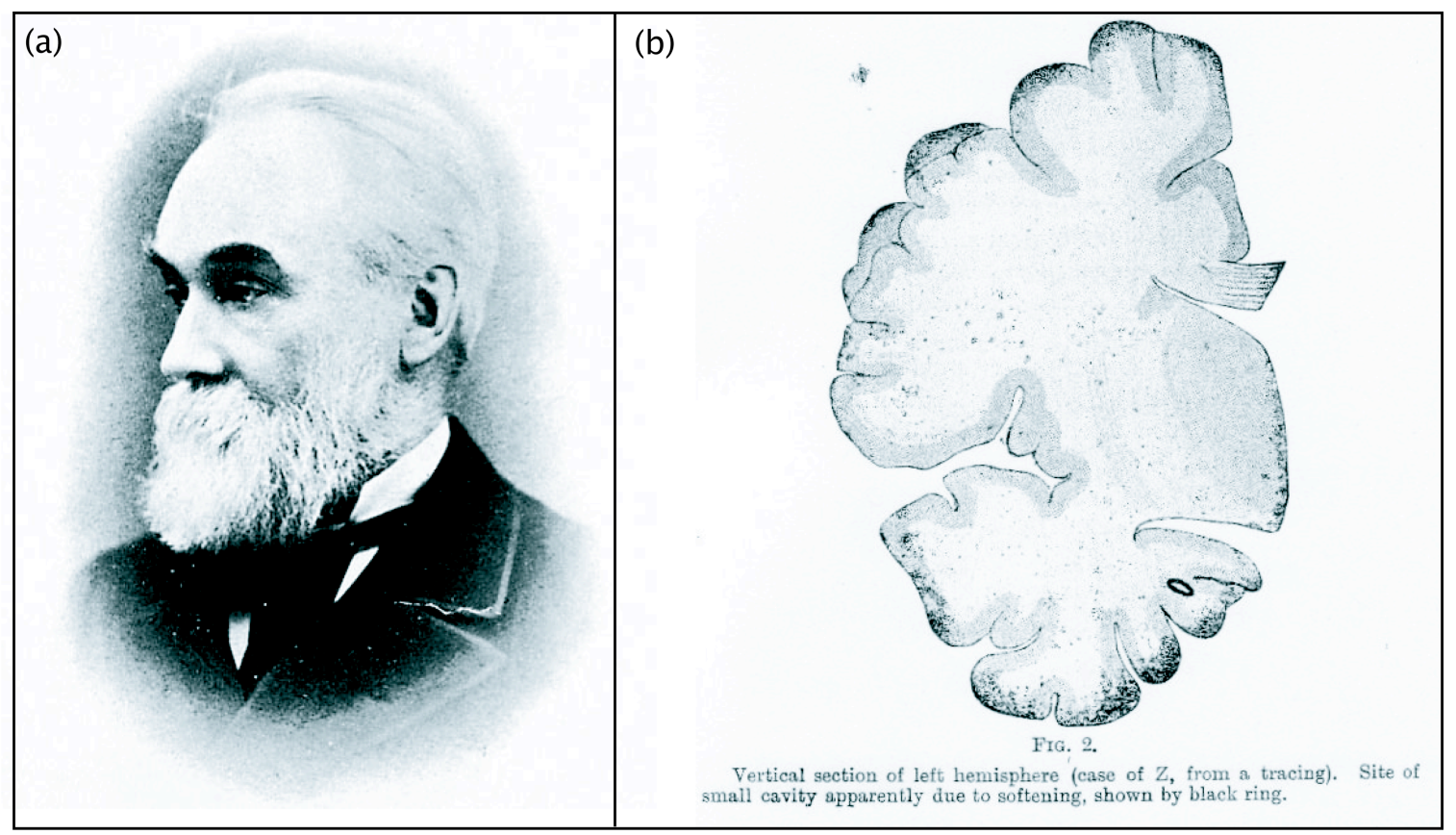

Figure 1.1: (a) John Hughlings-Jackson (1835 - 1911). (b) Illustration of pathological findings from the case of Dr Z. A "small patch of softening" was found in the left uncus (Hughlings-Jackson and Colman, 1898).

\subsubsection{Epilepsy as a cause of transient global amnesia}

The idea that transient, isolated memory loss may be the sole manifestation of epileptic seizures lay dormant in the scientific literature until the mid twentieth century, when debate began about the aetiology of the newly described syndrome of transient global amnesia (TGA) (Fisher and Adams, 1964). Patients with TGA experience the sudden onset of a profound amnesia that is not associated with any other focal neurological symptoms or signs and which resolves completely after just a few hours. Several early authors concluded that TGA was most likely due to cerebral seizure activity (Fisher and Adams, 1964). As discussed in section 1.4.1 below, it is 
now clear that this is not true for the majority of TGA attacks. Nevertheless, the following 25 years saw a steady trickle of case reports (for example, Deisenhammer (1981); Gallassi et al. (1986); Kapur (1990); Lou (1968); Morrell (1980); Pritchard et al. (1985); Stracciari et al. (1990); Tassinari et al. (1991)) describing patients in whom TGA-like episodes were found to be due to epilepsy (see Figure 1.2).

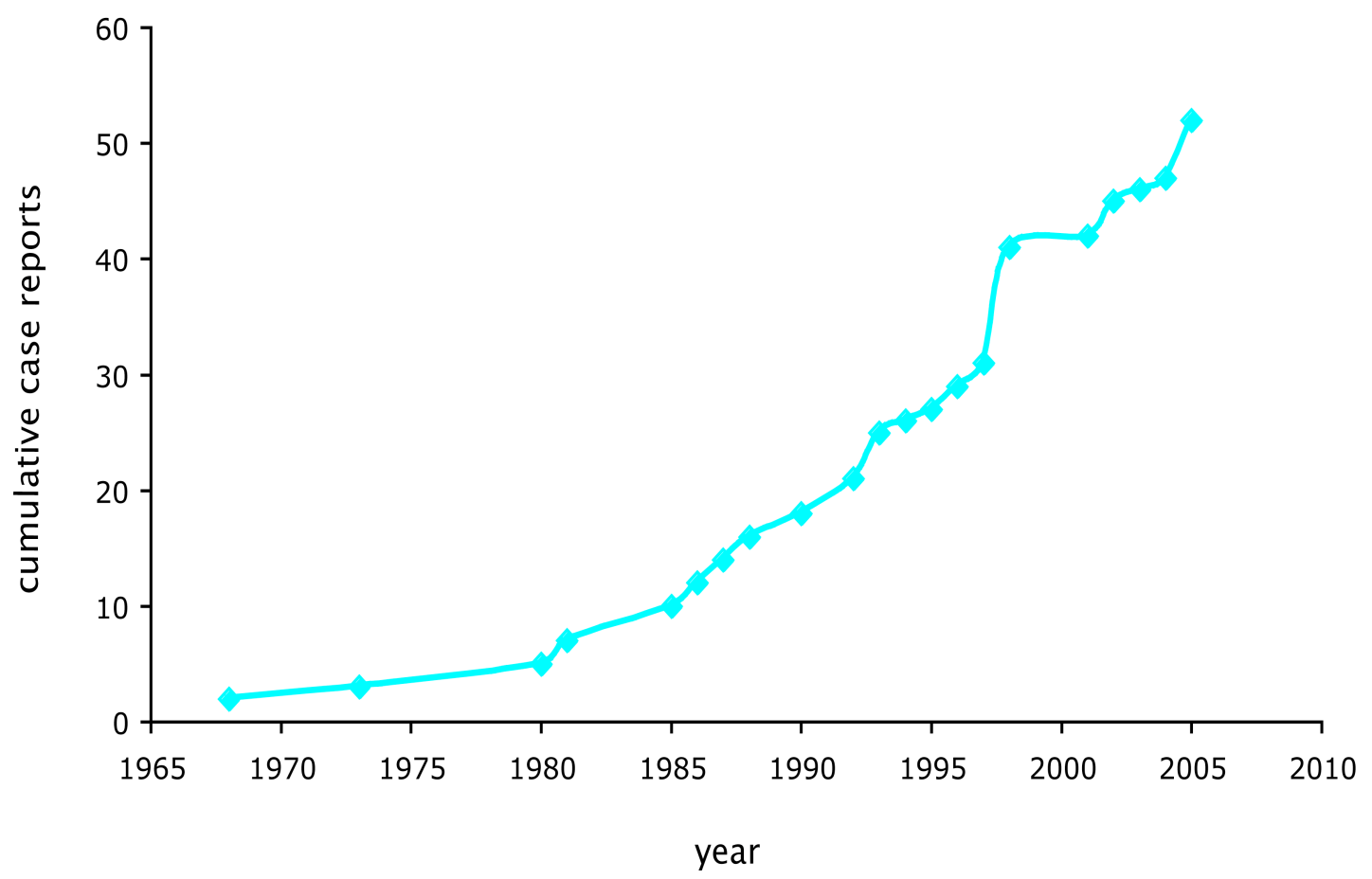

Figure 1.2: Graph showing cumulative number of cases meeting diagnostic criteria for TEA in the literature.

A variety of names have been applied to this phenomenon including epileptic amnesic attacks (Pritchard et al., 1985), epileptic transient amnesia (Stracciari et al., 1990), epileptic amnesia (Gallassi et al., 1990b), ictal amnesia (Rowan and Rosenbaum, 1991), pure amnestic seizures (Palmini et al., 1992) and transient epileptic amnesia (TEA) (Kapur, 1990). In the present work, I adopt the last of these in order to reflect the similarity of this condition to, but also its distinction from TGA. 


\subsubsection{Early characterisation of TEA as a distinct neurological entity}

In a large case-control study of TGA, Hodges and Warlow ((Hodges and Warlow, 1990a; Hodges and Warlow, 1990b), despite using stringent diagnostic criteria (see section 1.4.1), discovered that a significant minority (7\%) of the patients in their series went on to develop complex partial seizures. The authors comment that: "clearly not all cases of TGA are due to epilepsy, but the same indistinguishable clinical phenomenon may arise following complex partial seizures" (Hodges and Warlow, 1990b).

The term 'transient epileptic amnesia' was introduced in 1990 by Kapur in an essay in which he reviewed the published case reports, distilled the principle clinical features, compared them with those of TGA and concluded that TEA was a distinct neurological entity worthy of further study (Kapur, 1990). In particular, he noted that whilst some TEA attacks might be indistinguishable from TGA, they recur with greater frequency, are usually briefer, may involve a less dense amnesia, and often occur upon waking. Furthermore, patients may experience persistent memory difficulties: in particular, the loss of memories for remote, personally experienced events.

Diagnostic criteria for TEA were proposed by Zeman et al. (1998), who studied a series of 10 patients in whom:

1) there was a history of recurrent witnessed episodes of transient amnesia

2) cognitive functions other than memory were judged to be intact during typical episodes by a reliable witness

3) there was evidence for a diagnosis of epilepsy, provided by one or more of the following:

a. wake or sleep EEG

b. the co-occurrence of other seizure types (if their roughly concurrent onset and/or close association with episodes of transient amnesia suggested a connection)

c. a clear-cut response to anticonvulsant therapy. 
The authors report the clinical and neuropsychological features of these 10 patients and review 21 previous published cases. Their findings support and extend the earlier descriptions by Kapur (Kapur, 1990; Kapur, 1993b). All cases are briefly described in their paper, including the following one (case 3):

A 68-year old retired engineer gave an eight-month history of episodes of amnesia which usually occurred on waking, and involved extensive retrograde amnesia with incomplete anterograde amnesia for the episodes themselves. Apart from occasional repetitive questioning his behaviour was normal during the initial attacks. He sometimes complained of an odd taste or smell. Seven months after the first attack he had an episode of amnesia during which he was clearly confused and unable to dress himself; after this his wife described episodes in which he became inaccessible for a few minutes, with no subsequent recall of events during the episodes. During this period it became clear that he had developed an extensive patchy retrograde amnesia for events of the past 30 years. He had a history of myocardial infarctions seven and nine years ago. Brain CT was normal and SPECT showed minor reduction in uptake frontally. Wake EEG showed sharpened theta activity in both temporal lobes; sleepdeprived EEG showed independent bitemporal unequivocal epileptiform abnormalities. Treatment with sodium valproate abolished the episodes of overt amnesia.

The ten cases are summarised in Table 1.1.

Table 1.1 Zeman et al. (1998): Clinical, EEG, and neuropsychological features of 10 cases of TEA

\begin{tabular}{|c|c|c|c|c|c|c|c|c|c|c|c|c|}
\hline Case & Age & Sex & $\begin{array}{l}\text { History } \\
\text { (duration) }\end{array}$ & Attacks & $\begin{array}{l}\text { Attack } \\
\text { duration }\end{array}$ & $\begin{array}{l}\text { Rep. } \\
\text { qn. }\end{array}$ & $\begin{array}{l}\text { Sleep } \\
\text { related }\end{array}$ & $\begin{array}{l}\text { EEG } \\
\text { findings }\end{array}$ & $\begin{array}{l}\mathrm{Rx} \\
\text { response }\end{array}$ & $\begin{array}{l}\text { Other } \\
\text { epilepsy }\end{array}$ & $\begin{array}{l}\text { TEA } \\
\text { aura }\end{array}$ & $\begin{array}{l}\text { Focal } \\
\text { retrograde } \\
\text { amnesia }\end{array}$ \\
\hline 1 & 63 & $M$ & $3 y$ & 20 & $1 \mathrm{~h}$ & + & + & - & $+\mathrm{CBZ}$ & sps & $+/-$ & + \\
\hline 3 & 68 & $M$ & 8 months & 5 & h-days & + & + & +Bilateral & + SVP & sps,cps & - & + \\
\hline 4 & 66 & $\mathrm{~F}$ & 14 months & 5 & $<1 \mathrm{~h} 1 \mathrm{~h}$ & + & + & $+\mathrm{L}$ & E CBZ & - & - & - \\
\hline 6 & 70 & M & $2 y$ & 13 & $<1 \mathrm{~h}$ & + & + & Polyr & no $R x$ & sps & $+/-$ & - \\
\hline 7 & 73 & $M$ & 6 months & 5 & $1-2 \mathrm{~h}$ & - & + & Polyr & $+P$ & - & - & - \\
\hline 8 & 60 & $M$ & 6 months & 3 & $<1 h>=1 h$ & + & + & - & + SVP & $\mathrm{tcl}$ & - & + \\
\hline 9 & 69 & $M$ & 4 months & 3 & $<1 \mathrm{~h}$ & + & + & - & $+\mathrm{CBZ}$ & cps & $+1-$ & + \\
\hline 10 & 56 & M & 9 months & 20 & $<1 \mathrm{~h}$ & + & + & - & $+\mathrm{CBZ}$ & $\mathrm{cps}, \mathrm{tcl}$ & - & + \\
\hline
\end{tabular}


The mean age was 65 and all patients bar one were male. The amnesic episodes were usually brief, lasting less than one hour. During the episode, both anterograde memory - the ability to retain new information - and retrograde memory - the ability to retrieve previously stored information - were affected, although to varying degrees across individuals. All patients had experienced at least one attack upon waking. The average frequency was three episodes per year. Brain imaging was unremarkable in all but one patient, who had focal atrophy of the right hippocampus. Interictal EEG revealed epileptiform abnormalities over the temporal regions in four cases. The attacks generally responded well to anticonvulsant medication. The authors noted that vascular risk factors were common amongst the patients in their series and hypothesised that TEA might be caused by cerebrovascular disease. A battery of standard neuropsychological tests was administered and patients performed as well as control subjects in all domains. However, complaints of unusual, persistent memory difficulties were widespread. Two phenomena were particularly commonly described. Firstly, a number of patients reported that "their memories for recent personal events seemed to be intact initially, but may then disappear without trace". Secondly, seven of the 10 patients described a patchy impairment of remote memory, with loss of memories for salient autobiographical episodes, sometimes extending back over several decades. Zeman and colleagues hypothesise that TEA is a form of temporal lobe epilepsy (TLE), question whether the amnesic attacks are an ictal or postictal phenomenon and discuss possible mechanisms for the reported interictal memory difficulties.

Since the publication of the series by Zeman et al (1998), several more cases of TEA have been reported in the literature. A comprehensive review of all published cases meeting the diagnostic criteria is contained in Chapter 3.

\subsubsection{Related concepts}

Two concepts that have previously appeared in the medical literature are closely related to that of TEA. 


\section{The epileptic amnesic syndrome}

The 'epileptic amnesic syndrome' (EAS) is a concept used by Gallassi and colleagues to identify patients in whom memory disturbance, whether transient or persistent, is the presenting feature of TLE (Gallassi, 2006; Gallassi et al., 1990a; Gallassi et al., 1992; Gallassi et al., 1988a; Gallassi et al., 1988b; Gallassi et al., 1986). In a recent review (Gallassi, 2006), Gallassi summarises the clinical features of 30 patients with EAS whom he has seen over the past 20 years. The mean age is 58.6 years, with a range of 29 to 76 years. The predominant symptom is of memory complaints, beginning at the same time as seizures which are "stereotyped, characterised generally by short and subtle loss of contact and oral automatisms". It is noted that these seizures may remain undetected for a long time, and that many patients also experience "acute attacks of memory dysfunction". Although Gallassi does not report detailed clinical or neuropsychological data for the majority of cases, there is clearly substantial overlap with the phenomenon of TEA as described by Kapur (1993b) and Zeman et al. (1998). For TEA, however, the defining characteristic is transient amnesic attacks rather than general memory dysfunction as the presenting feature of epilepsy.

\section{Pure amnestic seizures}

Palmini et al. (1992) report a series of eight patients with known temporal lobe epilepsy and complex partial seizures and who, in addition, had episodes during which they behaved apparently normally but for which they were later amnesic. These episodes are termed 'pure amnestic seizures' (PAS). The patients were younger than the patients with TEA described by Zeman et al. (1998), having a mean age at onset of seizures of 15 years. The amnesic attacks described appear only to have affected anterograde memory. One patient had an episode whilst undergoing extracranial EEG recording and video monitoring. During a bitemporal electrographic seizure, she behaved entirely normally and had a brief telephone conversation with her cousin, but later had no recollection of the event. As a group, the patients showed some interictal impairment on standard tests of memory, but this was similar to a control group of patients with TLE but no pure amnesic episodes. The authors dismiss the possibility that the phenomenon might be mistaken for TGA: they emphasise the brevity of PAS, the lack of repetitive questioning and retrograde amnesia and the younger age range, 
and state that "all patients with PAS also have unmistakeable conventional complex partial seizures". Whilst these conclusions may be premature, they highlight the distinction between PAS and TEA. The report focuses on the potential anatomical substrate for PAS and suggests that seizure discharges result in bilateral functional deactivation of medial temporal structures, but spare the temporal neocortex by propagating from one hemisphere to the other across the dorsal hippocampal commissure.

\subsection{Persistent memory deficits in TEA}

\subsubsection{Memory impairment in epilepsy}

People with epilepsy frequently complain of memory difficulties. A community-based survey of over 1000 epilepsy patients in the United States revealed that cognitive difficulties ranked highest on a list of potential concerns (Fisher et al., 2000). In another study, $54 \%$ of over 700 people with epilepsy regarded memory problems as a moderate to severe nuisance (Thompson and Corcoran, 1992). Many interacting factors may affect memory function in patients with epilepsy including the underlying neuropathology (Lencz et al., 1992), seizure activity (Jokeit et al., 2001), anticonvulsant medication (Motamedi and Meador, 2004), surgery (Téllez-Zenteno et al., 2007), age (Lespinet et al., 2002), genetic background (Busch et al., 2007) and psychosocial factors (Elixhauser et al., 1999).

Complaints of memory dysfunction are particularly widespread in TLE, the most common form of adult-onset epilepsy, in which memory-related brain structures including the hippocampus are directly involved by seizure activity. It is well recognised that TLE may cause deficits on neuropsychological tests of memory, which typically assess the ability to retain new information over a delay of about 30 minutes (Hermann et al., 1997). The degree of impairment has been shown to correlate with pathological abnormalities (Oxbury and Oxbury, 1989; Pauli et al., 2006; Rausch and Babb, 1993), hippocampal atrophy on neuroimaging (Kilpatrick et al., 1997; Lencz et al., 1992; Reminger et al., 2004) and a number of clinical variables such as the age of onset of epilepsy, seizure frequency and lifetime number of seizures (Dodrill, 1992; Giovagnoli and Avanzini, 1999; Hendriks et al., 2004; 
Lespinet et al., 2002; Oyegbile et al., 2004). Furthermore, the laterality of the seizure focus has consistently been found to influence the type of material for which memory is most affected, with left TLE causing more pronounced deficits in verbal memory, and, less consistently, right TLE affecting non-verbal memory (Baxendale et al., 1998; Gleissner et al., 1998; Hermann et al., 1997). However, the severity of memory difficulty reported by patients with epilepsy, as with other types of neurological disease, correlates poorly with objective measures, and many patients perform at average or above-average levels (Piazzini et al., 2001).

The disparity between subjective and objective memory performance in TEA is, therefore, not unusual among patients with epilepsy. However, the memory deficits of which they complain - the accelerated forgetting of new information over days to weeks and the loss of memories for remote autobiographical events - are relatively novel and controversial. A small number of studies have investigated them in the context of epilepsy. These are comprehensively reviewed in Chapter 5. Here, by way of a brief introduction, I discuss two prominent studies that illustrate the phenomena in question and raise several important questions.

\subsubsection{Accelerated long-term forgetting (ALF)}

Blake et al (2000) investigated the long-term fate of memories for newly learned material in a group of 21 patients with focal epilepsy and 16 matched, healthy control subjects. Patients performed normally on a range of standard neuropsychological tests. Over several learning trials, participants memorised a short paragraph to a criterion of $90 \%$ correct. Recall was tested at delays of 30 minutes and eight weeks. The authors found that the group of patients with seizures originating in the left temporal lobe showed normal retention after 30 minutes but dramatic loss of memory over the subsequent weeks. Patients with right TLE or extra-temporal epilepsy did not exhibit this phenomenon. There was no correlation of this long-term forgetting rate with scores on standard memory tests, seizure frequency or mood. Blake and colleagues propose that accelerated forgetting may partially explain the discrepancy between subjective complaints of memory difficulties in epilepsy and performance on standard neuropsychological instruments, which typically test retention over intervals of up to one hour. They also highlight the potentially important consequences of their 
findings for memory theory: their results demonstrate that, following acquisition, memories have an extended period of vulnerability. Accelerated forgetting might then be interpreted as a deficit in long-term memory consolidation. This study raises questions about the prevalence of the phenomenon amongst patients with epilepsy, about the underlying neurophysiological mechanisms and about its potential for treatment. These issues are discussed more fully in section 1.6 below and in Chapters 4 and 5.

\subsubsection{Autobiographical memory loss}

The autobiographical amnesia typically reported by TEA patients was explored in depth in a detailed case study by Manes et al (2001). The patient, RG, was a 68-year old man who experienced five episodes of transient amnesia over a period of eight months. Interictal EEG revealed bilateral epileptiform abnormalities over the temporal region, and the MRI brain scan showed white matter changes consistent with small vessel cerebral ischaemia, but no cortical abnormality. Since the onset of the attacks, RG and his wife had become aware of persistent gaps in his memory, extending many years into the past. This was particularly noticed with regard to salient, personally experienced episodes such as family holidays. RG performed normally on a battery of standard neuropsychological tests of memory, visuospatial perception, language and executive function. However, examination of his autobiographical memory revealed a profound deficit in his recollection of personally experienced events from the past 30 years (see Figure 1.3).

Earlier memories were comparatively spared, as was factual knowledge about his life, famous faces, famous names, famous places and public events. The authors hypothesise that this extensive retrograde amnesia might be due to degradation or erasure of neocortically based memory representations. They indicate that further study in a larger group of patients with TEA is necessary to address questions regarding the epidemiology and pathophysiology of autobiographical amnesia. I explore these issues in more detail in section 1.6 below and in Chapters 4 and 5 . 


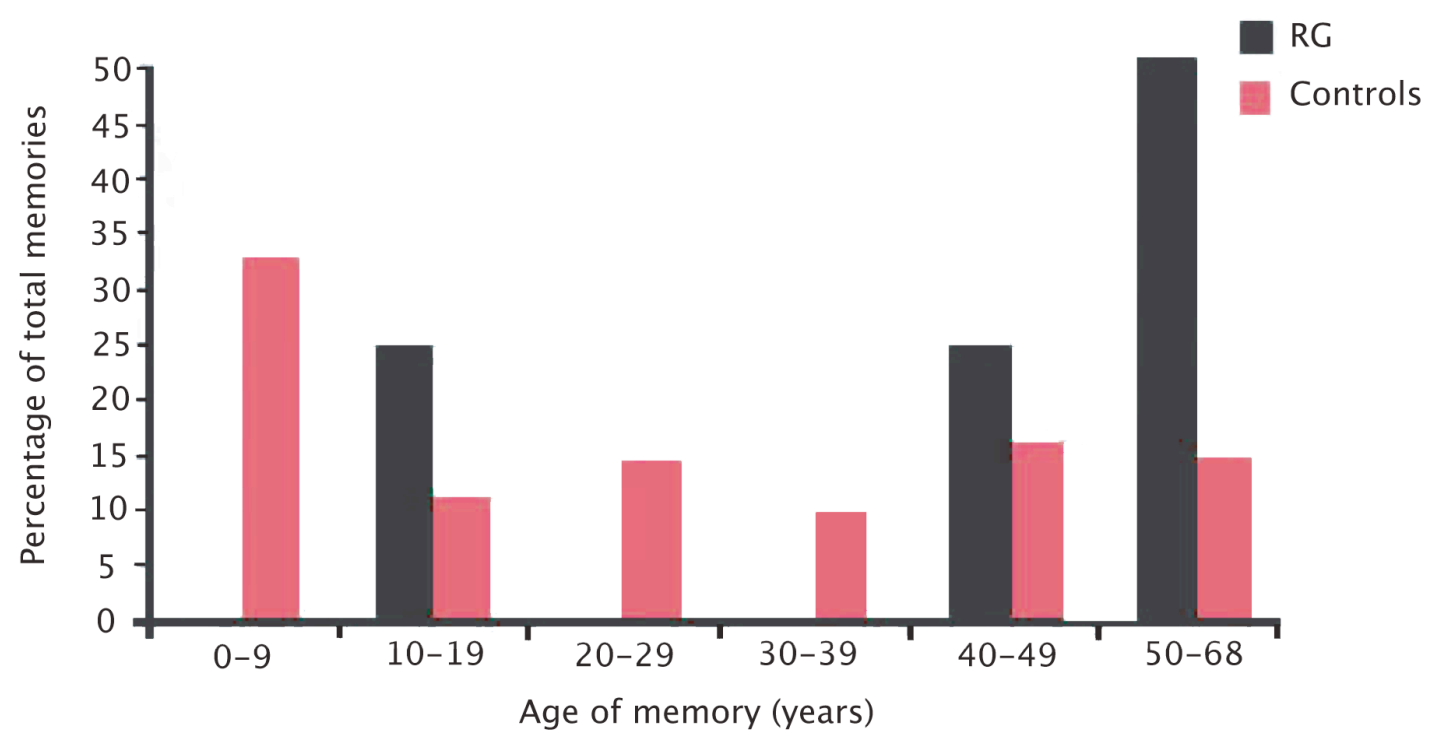

Figure 1.3: Comparison of the memories produced by RG and controls on the modified Crovitz test (Manes et al., 2001). In this test, subjects attempt to provide episodic memories in response to keywords, such as 'boat', for specified life epochs. The figure shows the age of memories by decades as a proportion of the total memories produced. RG predominantly retrieved memories from the distant past.

\subsection{Syndromes of transient amnesia}

In this section, I review the differential diagnosis of transient amnesia. Amongst the conditions discussed, TGA is probably the most common and widely studied, although its pathophysiology remains unknown. Transient amnesia can also result from psychogenic mechanisms. As will be discussed below, the clinical profile in such cases is very different from that seen in organic amnesia. In addition, a period of isolated memory loss may follow mild head injury or be caused by certain medications including benzodiazepines and anticholinergics.

\subsubsection{Transient global amnesia}

The term 'transient global amnesia' was coined in 1964 by Fisher and Adams in a paper describing the clinical features of 17 patients (Fisher and Adams, 1964), 
although several authors published accounts of similar cases using different terminology at around the same time (Bender, 1960; Evans, 1966; Guyotat and Courjon, 1956; Poser and Ziegler, 1960). It has been suggested (Hodges, 1991) that, prior to this period, the syndrome remained buried within the literature on psychogenic, or hysterical amnesia.

\section{$\underline{\text { Illustrative case description }}$}

A 63-year old, recently retired teacher was brought to the Accident and Emergency department by her husband. One hour earlier, she had telephoned him from the local gym where she had just finished her daily workout and said: "I don't know where I am. What's happening? Where am I? " Despite his reassurances, she had continued to repeat the same questions. On examination, she was disoriented in time and place, had no recollection for events of the previous week and was unable to retain new information - including the identity of the attending doctor. Besides the amnesia, there were no other neurological signs or symptoms. A CT scan of the head was normal. Over the following six hours, her memory deficit gradually resolved although she was left with a dense 'gap' for the episode of transient amnesia itself and for the preceding trip to the gym.

\section{Clinical features}

The most widely accepted diagnostic criteria for TGA were introduced by Hodges and Warlow (Hodges and Warlow, 1990b):

1. attacks must be witnessed and information available from a capable observer who was present for most of the attack

2. there must be a clear-cut anterograde amnesia during the attack

3. clouding of consciousness and loss of personal identity must be absent, and the cognitive deficit must be limited to amnesia (that is, no aphasia, apraxia, etc)

4. there should be no accompanying focal neurological symptoms during the attack and no significant neurological signs afterwards

5. epileptic features must be absent

6. attacks must resolve within 24 hours 
7. patients with recent head injury or active epilepsy (that is, remaining on medication or one seizure in the past two years) are excluded.

TGA is a relatively infrequent occurrence, having an annual incidence of between three and ten per 100,000 (Hodges, 1991). Nonetheless, it has attracted considerable attention in the scientific literature and circa 1500 cases have been described (Quinette et al., 2006). The mean age of onset is 62 years and the condition occurs almost exclusively in individuals between the ages of 40 and 80 (Hodges, 1991). In younger people, a similar phenomenon may occur following head injury (Haas and Ross, 1986) but these are usually excluded from the rubric of TGA. A recent review of all literature cases (Quinette et al., 2006) failed to find any significant difference in frequency between the sexes.

Ever since the early reports, it has been noticed that TGA is often preceded by a period of intense emotional or physical stress. Frequently reported triggers include immersion in cold water, sexual intercourse, receipt of distressing news or a heated argument. The onset of anterograde amnesia is betrayed by repetitive questioning, often related to attempts at self-orientation such as "What day is it?" or "What am I doing here?" Although a small amount of information can be retained for a few seconds, it is rapidly lost when the patient's attention shifts. The retrograde amnesia may cover a few hours prior to the attack onset or be much more extensive. Witnesses usually describe the patient as 'confused', but careful examination reveals that there is no impairment of conscious level or of other cognitive functions such as attention, language or perception. In contrast to psychogenic forms of amnesia, knowledge of personal identity is always retained. Non-specific symptoms such as headache, nausea or dizziness may be present, but there are no focal neurological deficits. Symptoms usually resolve gradually over four to ten hours, with recovery of retrograde memory occurring more rapidly than anterograde memory (Kapur et al., 1998). The majority of patients, therefore, do not present to a neurologist in the acute phase. After recovery, a dense amnesic gap for the attack itself persists. There is no clinically significant, long-term cognitive impairment, although more subtle deficits have been reported. Recurrence is rare and occurs at a rate of around three to five percent per year. 


\section{Aetiology}

Ever since the first descriptions of TGA, there has been considerable debate about its aetiology and this debate remains unresolved. TGA-like attacks have been reported in association with brain tumours, sodium amobarbital injection, high altitude, herpes simplex encephalitis and the use of marijuana. This suggests that TGA could be a 'final common pathway' with numerous potential triggers.

Epilepsy: Fisher and Adams (1964) believed that the most likely explanation for TGA was cerebral seizure activity and several other early authors also maintained this position (Cantor, 1971; Deisenhammer, 1981; Gilbert, 1978; Godlewski, 1968; Lou, 1968; Rowan and Protass, 1979; Tharp, 1969). This idea has now largely fallen out of favour for a number of reasons. Epilepsy is, by definition, a recurrent condition whereas patients usually experience only a single episode of TGA in their lifetime. The duration of most TGA attacks is also uncharacteristically long for epileptic seizures. Other common features of generalised or partial epilepsy are absent during the attack. Finally, EEG recordings during or after a TGA attack are almost invariably normal or show only minor non-specific abnormalities (Jacome, 1989; Miller et al., 1987). As mentioned above, in a series of 114 patients followed up over a mean of 34 months following TGA, Hodges and Warlow identified a small subset (7\%) of patients who subsequently developed epilepsy (Hodges and Warlow, 1990a). These patients, in keeping with more recent descriptions of transient epileptic amnesia (see below), typically had briefer and recurrent amnesic episodes.

Migraine: Several authors have proposed a causal link between migraine and TGA (Caplan, 1981; Gilbert and Benson, 1972; Laplane and Truelle, 1974; Olivarius and Jensen, 1979). A past history of migraine has been reported to be present in up to $30 \%$ of patients with TGA (Hodges and Warlow, 1990b), a significantly higher proportion than in control subjects, and migrainous features, particularly headache and nausea, accompany about 20\% of TGA attacks (Hodges, 1991). Olesen and Jorgensen suggested that the underlying pathological mechanism behind both TGA and migraine might be the experimentally observed phenomenon of spreading depression (Olesen and Jorgensen, 1986). A range of stimuli, applied directly to the cortex, may induce a wave of depolarisation that spreads at a rate of three to five millimetres per minute 
and reduces cerebral blood flow for a period of about one hour. Spreading depression can be elicited in the hippocampus of experimental animals in which it provokes a transient period of amnesia (Avis and Carlton, 1968). According to the migraine hypothesis, emotional or physical stressors lead to the release of glutamate in the hippocampus triggering spreading depression and hippocampal dysfunction. Critics argue that the migraine hypothesis does not readily account for the age range of TGA or its low recurrence rate (Pantoni et al., 2000).

Arterial: Permanent amnesia can result from stroke, most frequently when involving the thalamus bilaterally (Schmahmann, 2003). It is therefore reasonable to ask whether TGA is a form of transient ischaemic attack (TIA). A number of studies, however, have shown that patients with TGA have fewer vascular risk factors than control subjects with TIA and a more favourable prognosis in terms of mortality and cerebrovascular events (Hodges and Warlow, 1990b; Quinette et al., 2006). Recent imaging studies (see below) have shown changes in the hippocampus in the days following attacks but the features are not typical of standard ischaemic stroke.

Venous: A more recent theory is that originated by Lewis (Lewis, 1998), which postulates that the well-recognised triggers of TGA lead to an increase in central venous pressure. In susceptible individuals, this should cause venous ischaemia in medial temporal and diencephalic brain regions. Support for this idea comes from reports of jugular valve insufficiency leading to retrograde venous flow during a Valsalva-like manoeuvre in $73.4 \%$ of TGA patients compared with $35.7 \%$ of controls (Sander and Sander, 2005).

Psychological: Emotional stress as a trigger for TGA is well recognised (Brown, 1998; Inzitari et al., 1997; Quinette et al., 2006). It has been reported that TGA patients are more likely to have phobic personality traits (Inzitari et al., 1997) and a past history and family history of psychiatric disease (Pantoni et al., 2005) than normal control subjects and patients with TIAs. These authors propose that, in such individuals, hyperventilation in response to a stressful situation leads to a reduction of cerebral blood flow in medial temporal regions and consequent transient amnesia (Pantoni et al., 2000). 


\section{Neuroimaging}

For many years, structural brain imaging was thought to be entirely normal in the majority of TGA cases. Recently, however, a number of studies have shown that, in the period immediately following a TGA attack (within 24 to 72 hours of onset), diffusion weighted magnetic resonance imaging can detect small (one to two $\mathrm{mm}$ ), punctate hippocampal lesions, indicating areas of restricted diffusion, in about $70 \%$ of patients (Bartsch et al., 2006). These lesions are most frequently found in the CA1 subfield of the hippocampus, a region known to be particularly sensitive to hypoxic injury, and may be unilateral or bilateral. At repeat scanning four to six months later, the lesions are no longer detectable. The pathophysiology of these abnormalities remains unclear although they have been used to support hypotheses of both arterial and venous aetiologies (Sander and Sander, 2005; Winbeck et al., 2005).

\section{Neuropsychology}

Formal neuropsychological testing during a TGA attack bears out the clinical impression of an isolated deficit of long-term memory. The patient is able to hold and manipulate information normally in working memory as tested, for example, by backwards digit span (Quinette et al., 2003). However, on tests of long-term anterograde memory, such as delayed recall of a word list, story or complex figure, performance is at floor (Hodges, 1991). Interestingly, despite having no recollection of stimuli encountered during the attack, patients nonetheless demonstrate perceptual priming (Kapur et al., 1996a) suggesting that, as with more permanent amnesia from medial temporal lobe or diencephalic damage, implicit memory remains intact.

During the episode, patients also lose access to memories acquired prior to the onset of the attack (retrograde amnesia) to some degree. The initial acquisition of such memories is, of course, beyond the reach of experimental manipulation so assessment of retrograde amnesia can be difficult. In the acute and recovery phase of TGA, tests probing memory for both personal and public facts and events have revealed variable patterns of impairment across individuals. In general, accounts of personally experienced episodes are "curiously empty and lacking in colour, as if reduced to the bare bones of memory" (Hodges and Ward, 1989). They lack what has been called "autonoetic consciousness" - the feeling of having experienced the past episode 
oneself (Wheeler et al., 1997). Retrograde amnesia may affect memories from across the lifespan (Guillery-Girard et al., 2004) or from a more limited time period prior to the attack (Kritchevsky, 1997).

During the recovery phase, retrograde memory improves more rapidly than anterograde memory. In some cases, memories are recovered in chronological order whereas in others more salient, detailed memories return first no matter what age they are (Guillery-Girard et al., 2004; Kapur et al., 1998). Anterograde memory impairment lasts much longer than is clinically apparent. Deficits, particularly in story recall, can be demonstrated several days and, in some cases, several months later. Following recovery from TGA, patients are left with complete amnesia for events that occurred during the attack. There is also usually a short, permanent retrograde amnesia for events that occurred in the one to two hours leading up to the attack (Hodges, 1991).

\subsubsection{Psychogenic amnesia}

Cases of transient amnesia straddle the border between psychiatry and neurology, and highlight just how artificial and misleading the traditional distinction between these two disciplines can be. As discussed above, TGA, generally thought of as a 'neurological' condition with hints of 'organic pathology' in the medial temporal lobes, can be triggered by psychological stressors and may even be more common amongst people with certain personality traits. Other instances of transient amnesia, in which there is no obvious neuropathological explanation, may be more easily explained in purely psychological terms. Such cases, variably termed 'psychogenic amnesia', 'functional amnesia', 'dissociative amnesia' or 'hysterical amnesia', are characterised by:

"a memory loss that is attributable to an instigating event or process that does not result in insult, injury or disease affecting brain tissue, but that produces more forgetting than would normally occur in the absence of that instigating event or process" (Schacter and Kihlstrom, 1989). 
Psychogenic amnesia may be divided into two main subtypes after Kopelman (Kopelman, 2002b): situation-specific psychogenic amnesia and global psychogenic amnesia. In the former, there is a temporally circumscribed amnesia for a specific and usually emotionally charged event such as a criminal offence. The degree of amnesia is proportional to the violence of the offence, and up to $30 \%$ of convicted homicide cases have amnesia claimed at trial (Pyszora et al., 2003). Rates are higher in socalled 'crimes of passion', where a murder is unpremeditated and associated with extreme emotional arousal, and if alcohol or drug intoxication is involved. Although the mechanism of this type of amnesia is unclear, it is unlikely to be due to malingering, or "putting it on", and in many legal systems, amnesia does not in itself constitute a defence. On occasions, the defendant may claim decreased responsibility for a crime on account of an 'intrinsic' brain disorder such as epilepsy, sleepwalking or hypoglycaemia. Although such conditions are only very rarely implicated in violent crime, they can result in amnesia and pose considerable diagnostic challenges.

Global psychogenic amnesia is not restricted to a single event but involves memory loss for a large swathe of the patient's life.

\section{$\underline{\text { Illustrative case description }}$}

A 43-year old construction worker was brought to hospital by colleagues. That morning, he had suffered a minor head injury when his forklift truck collided, at low speed, with an earth bank. Since then, he had a "complete loss of memory", with no recollection of any past events. He was unable to remember his own name and failed to recognise his colleagues or, when she arrived, his recently estranged wife. Despite this, there was no apparent difficulty in learning new information - he could recount in detail the events following his arrival at hospital. MRI of the brain was normal. It later emerged that, since an acrimonious separation from his wife, he had been showing signs of depression and drinking heavily. He had suffered a period of concussion following a motorcycle accident in his 20 's.

The onset typically follows a stressful experience, such as a marital or financial crisis, and there is often a background of depression or alcohol abuse (Kritchevsky et al., 2004). Psychogenic amnesia is commonly associated with a history of 'organic' transient amnesia (Berrington et al., 1956). Knowledge of personal identity is often 
impaired: this is not a feature of organic amnesia. There may be a period of wandering, 'psychogenic fugue' that typically lasts for a few hours or days. There is usually a relative preservation of anterograde memory, so that patients are able to 'relearn' about themselves, although they may complain that such memories lack the experiential aspect, or 'autonoetic consciousness', that defines true episodic memory. Recovery is frequently protracted and incomplete. The brain mechanisms responsible for psychogenic amnesia are unknown. It has been proposed that there is a functional disconnection between memory storage and retrieval mechanisms in the frontal and temporal lobes (Markowitsch, 2003) and functional neuroimaging studies have revealed decreased brain metabolism, at baseline and during attempted memory retrieval, in the right frontal cortex (Markowitsch, 1999).

\subsubsection{Head Injury}

Following closed head injury, there is often a period of posttraumatic amnesia (PTA) during which new learning is grossly impaired and there is retrograde memory loss for events leading up to the head injury. This can occur even in mild injuries which produce no coma. The retrograde amnesia gradually shrinks until the patient can recall all but the brief instant preceding the head injury. The duration of the anterograde amnesia is predictive of the final neuropsychological outcome (Levin et al., 1979). The memory deficits are usually accompanied by a variety of other cognitive and behavioural problems including impaired attention, agitation, lethargy and disinhibited behaviour. The pathological mechanisms underlying PTA may involve a combination of focal contusions, characteristically in the frontal or temporal lobes, diffuse axonal injury and secondary effects of hypoxia or ischemia. Sometimes, a mild head injury, such as sustained during sport, may trigger a TGA-like episode with repetitive questioning. This tends to occur in people below the usual age range for TGA, and may be recurrent (Haas and Ross, 1986).

\subsubsection{Drugs}

A period of transient memory impairment can also result from administration of several types of drug including benzodiazepines, anticholinergics, ketamine (an Nmethyl-D-aspartate (NMDA) receptor antagonist) and alcohol. Benzodiazepines, 
particularly midazolam, are widely used in clinical practice as an adjunct to or even replacement of anaesthesia. The resultant, short-lived and predominantly anterograde amnesia is thought to result from specific impairment of memory encoding, not just a generalised reduction in alertness (Curran and Birch, 1991). Retrograde memory is unaffected. Benzodiazepines bind to the inhibitory $\gamma$-aminobutyric acid A $\left(\mathrm{GABA}_{\mathrm{A}}\right)$ receptor and increase its affinity for GABA. The specificity of the cognitive deficit may be partially due to the abundance of these receptors in the hippocampal complex. Declarative, conscious memories appear to be specifically targeted: certain types of procedural memory and perceptual priming have been shown to remain intact (Arndt et al., 2004; Thomas-Antérion et al., 1999).

\subsection{The amnesic syndrome}

The opportunity to perform detailed assessment of cognitive function during an episode of transient amnesia is a rare and serendipitous privilege. Understanding such episodes therefore partially involves extrapolation from studies of patients with more permanent lesions. In this section, I describe the neuropsychological characteristics of the classical amnesic syndrome and discuss its principle neurological causes.

\subsubsection{Characteristics of the amnesic syndrome}

The neuroscience of memory was revolutionised in the middle of the last century by Scoville and Milner's report of the patient H.M. who became densely amnesic following a bilateral temporal lobectomy for treatment of his epilepsy (Scoville and Milner, 1957). Just as case reports by Paul Broca and Karl Wernicke had uncovered the neurological basis of language 70 years earlier, so the study of H.M. revealed the critical role of the hippocampus and associated medial temporal regions in memory (see Figure 1.4).

Lacking these brain regions, H.M. was no longer able to retain new information for more than a few seconds once his attention had been briefly diverted to a different subject: that is, he showed anterograde amnesia. Furthermore, he was unable to recall events that had occurred during at least the three years preceding his operation: i.e. he had a temporally graded retrograde amnesia. Besides these memory deficits, his 
abilities in other areas of cognition including language, perception and executive function were strikingly preserved.

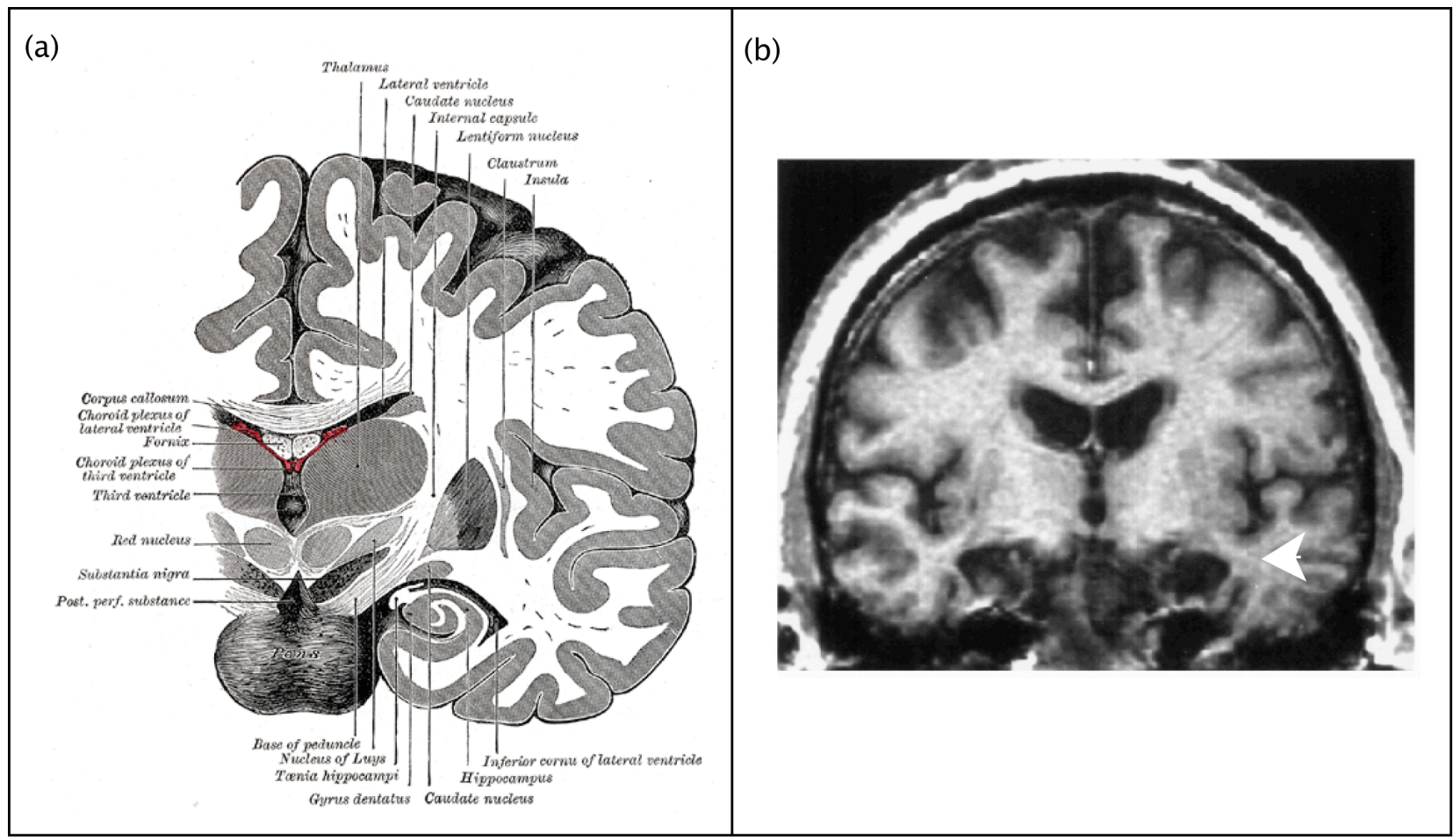

Figure 1.4: (a) Illustration showing the location of medial temporal lobe structures on coronal section (taken from Gray's Anatomy). (b) Coronal T1-weighted MRI scan of HM's brain, showing the absence of the hippocampus and related medial temporal lobe structures bilaterally (arrowhead).

Careful examination of the amnesia exhibited by H.M. and other patients has revealed some critically important distinctions between different types of human memory. Amnesics demonstrate normal performance on tests of working memory (Cave and Squire, 1992; Parkin and Leng, 1993). This ability to hold and manipulate information 'online' is central to the performance of many cognitive tasks including language comprehension, calculation and planning. Patients with amnesia are also able to retrieve 'overlearned' semantic memories such as knowledge of word meaning and 
perceptual content. Finally, they show preservation of skill learning, classical conditioning and perceptual priming, the bias towards or facilitation of processing of a stimulus caused by prior exposure to that same or a related stimulus (Squire et al., 1993). These forms of memory have in common the fact that they can be expressed without the need for explicit, or conscious, reference to the original learning event, and are often grouped together under the title of 'non-declarative' memory. Amnesic patients, therefore, show isolated impairment of 'declarative' memory - conscious memory for facts and events.

\subsubsection{Causes of the amnesic syndrome}

The amnesic syndrome is associated with a number of neurological conditions that affect the medial temporal lobes and connected areas including the diencephalon and basal forebrain (see Figure 1.5)

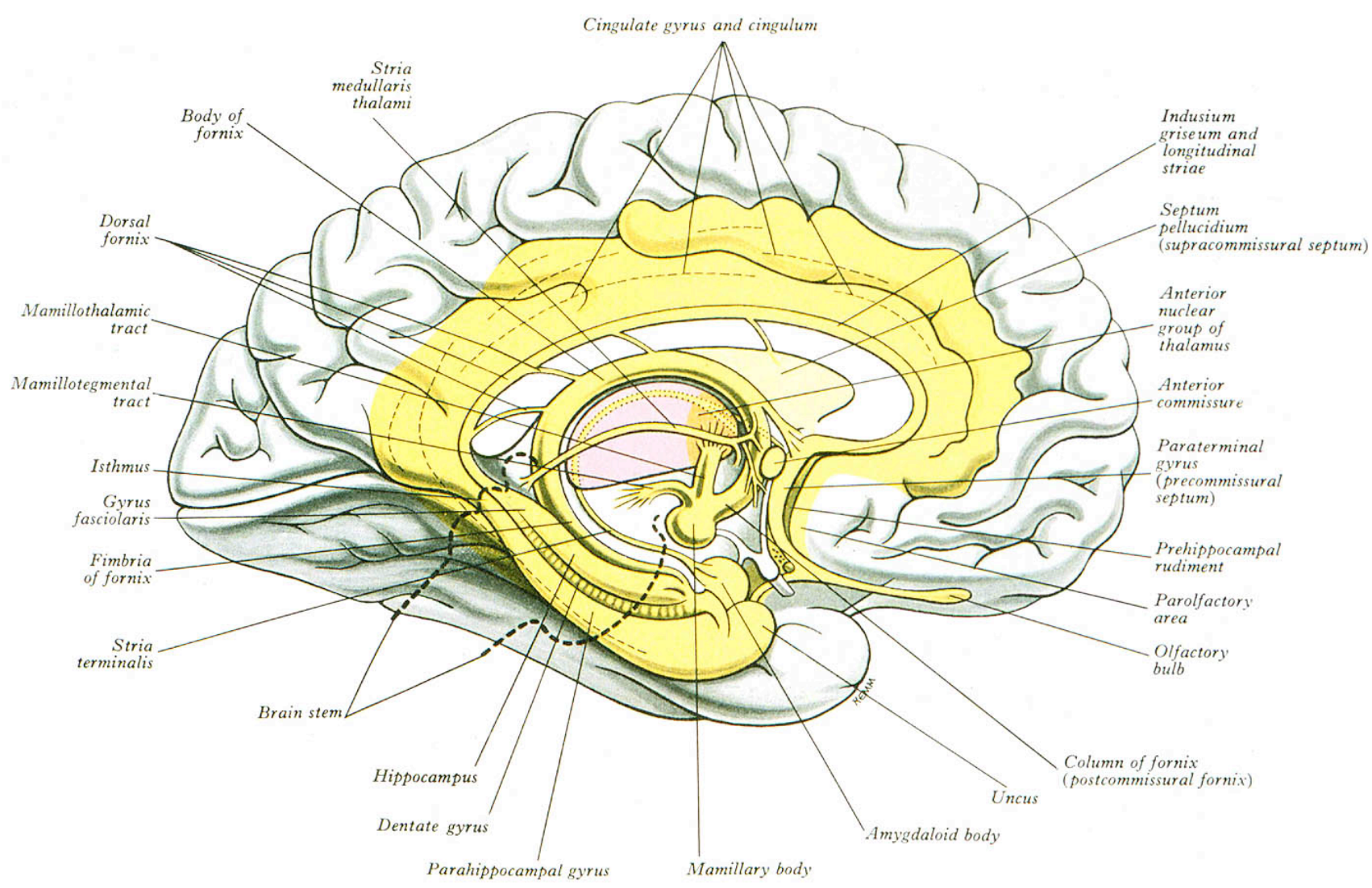

Figure 1.5: Diagram showing the anatomical relationship of human brain regions damage to which can result in the amnesic syndrome. 


\section{Bilateral medial temporal lobe damage}

Limbic encephalitis, a syndrome in which inflammation selectively targets the medial temporal lobe, may be caused by herpes simplex infection, by antineuronal antibodies as part of a paraneoplastic reaction or, as has recently emerged, by antibodies to voltage-gated potassium channels (Vincent et al., 2004). Medial temporal lobe damage may also be the most prominent consequence of cerebral anoxia occurring, for example, with cardiac arrest, carbon monoxide poisoning or birth injury. The mechanism of damage is multifactorial, but the excitotoxic effects of excessive glutamate and aspartate release are thought to play an important role (Caine and Watson, 2000).

\section{Korsakoff's syndrome}

Korsakoff's syndrome (KS) results from nutritional deficiencies usually associated with chronic alcohol abuse. The main foci of pathology are the dorsomedial nucleus of the thalamus and the mamillary bodies, although the amnesic deficit itself has recently been attributed to pathology in the anterior thalamic nuclei (Harding et al., 2000). Patients with KS have profound and global learning difficulties as well as problems with memory retrieval (Verfaellie and Cermak, 1991). These are frequently associated with additional features including confabulation, perseveration and executive dysfunction (O'Connor and Verfaellie, 2002).

\section{Stroke}

The territory supplied by the posterior cerebral artery (PCA) includes the posterior hippocampus and parahippocampal regions. Ischaemic stroke due to bilateral PCA occlusion can result in an isolated amnesic syndrome (Benson 1974), although additional deficits including visual field defects, alexia, colour agnosia and anomia may be present (Benson 1974). Amnesia has also been documented after thalamic strokes (Graff-Radford 1990), although the severity of the memory deficit varies in relation to the site of damage within the thalamus. A recent review (Van der Werf et al., 2000) concluded that damage to the mamillo-thalamic tract or anterior thalamic nuclei was both necessary and sufficient for an amnesic presentation. 


\section{Anterior communicating artery aneurysm rupture}

Memory deficits are common following aneurysmal rupture of the anterior communicating artery. The artery supplies the basal forebrain, anterior cingulate gyrus, anterior commissure, anterior columns of the fornix, and the genu of the corpus callosum. Memory deficits range from mild to severe and are typically characterised by difficulties with strategic retrieval, so that recognition memory may be comparatively spared. There are often accompanying behavioural abnormalities including lack of insight, confabulation and impaired executive function (D'Esposito et al., 1996). The neural substrate of the amnesia is not yet clear. However, several basal forebrain nuclei, including the septal nuclei and nucleus accumbens, are important sources of cholinergic input to the hippocampus as well as other cortical regions. Damage to these nuclei, together with the executive dysfunction caused by extension of the lesion along the medial frontal lobes, have been postulated as the primary causes of amnesia in these patients (Volpe et al., 1984).

\subsection{Neuroscientific theories of memory}

\subsubsection{Human memory systems}

It is widely held that the human brain supports a number of distinct memory 'systems'. Such systems are distinguished by their operational rules, neuroanatomical basis and, presumably, evolutionary history, rather than purely by the type of information processed by them (Schacter and Tulving, 1994). A simple and widely accepted classification of these memory systems, together with the brain regions thought to support them, is shown in Figure 1.6 (Squire and Zola-Morgen, 1996).

The declarative memory system is, at least initially (see section 1.6.3), dependent upon the medial temporal lobes and diencephalon, whilst memory for skills (procedural memory) and habits is supported by a variety of other brain regions including the basal ganglia and cerebellum. 


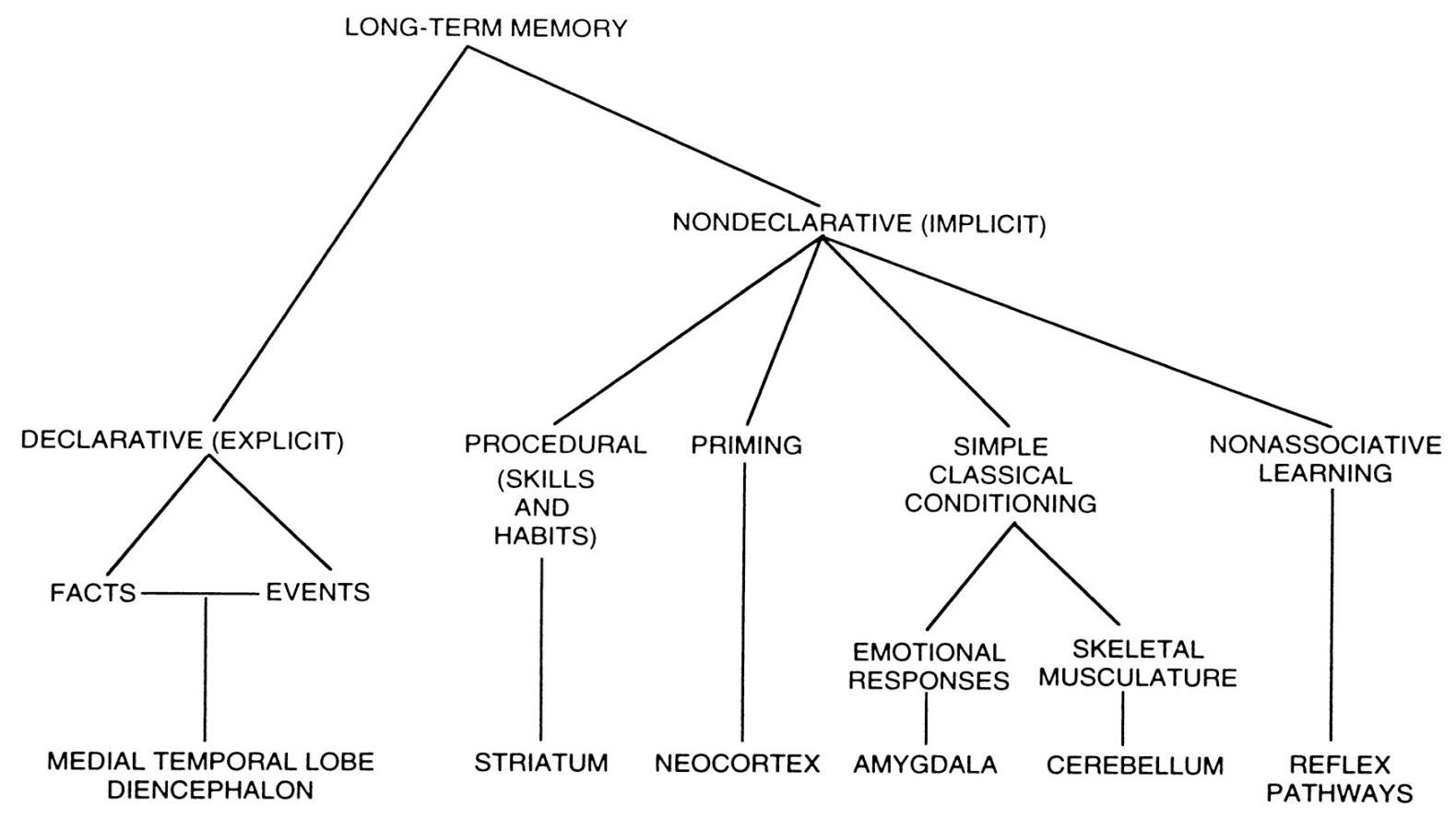

Figure 1.6: A taxonomy of long-term memory systems together with specific brain structures involved in each system (after Squire and Zola-Morgan (1996)).

As intimated in Figure 1.6, a further separation is often made within declarative memory between memory for events, episodic memory, and memory for facts, semantic memory. This distinction, originally made by Endel Tulving (Tulving, 1972), emphasises that episodic memories - for example, a memory of meeting a famous person - are acquired at a single learning experience whose spatiotemporal context is an intrinsic feature of the memory, whereas semantic memories - for example, knowledge about why that person is famous - are generally learned over multiple occasions and are recollected in a context-independent fashion. Furthermore, Tulving and others have defined 'true' episodic memory in terms of an intrinsic recollective experience - 'autonoetic consciousness' - the experience of 'mental time travel' (Tulving, 1983; Tulving, 2002; Wheeler et al., 1997). Thus, whilst various animals may have memories that are context-specific (Clayton and Dickinson, 1998; Clayton et al., 2001), it has been postulated that true episodic memory may be a 
uniquely human property. Whether or not episodic and semantic memory are subserved by different memory systems in the brain has proved controversial. Some authorities have contended that episodic memories are particularly dependent upon the hippocampal complex, which supports their necessary spatial elements or perhaps other highly relational features (Burgess et al., 2002), whilst semantic memory functions are served by lateral temporal neocortex (Vargha-Khadem et al., 1997). Others, however, regard the two as equally dependent upon the medial temporal lobe, whose principle role is the encoding and consolidation of all declarative memories (Squire et al., 2004). It should be noted that the concept of distinct memory systems continues to be challenged from certain directions, particularly from those who hold that the principle role of the hippocampus is in spatial processing and that memory deficits arising from hippocampal damage are a result of problems with spatial processing rather than memory per se (Gaffan, 2001; Lee et al., 2005).

Memory function can be conceptualised as having at least three components: encoding, storage and retrieval. Encoding a declarative memory involves the initial selection and processing of perceptual inputs that results in a memory 'engram'. This hypothetical engram is a mental or neural representation that provides the causal link between initial experience and later remembering. The information that is encoded during any event depends upon many factors including the way that attentional resources are deployed, the semantic and emotional content of the event and the prior experience of the subject. Following encoding, the engram is stored over a period during which it may undergo modifications including 'consolidation', discussed in the following section. Finally, at retrieval, voluntary search strategies or subconscious recognition mechanisms succeed in reactivating the engram and producing a memory. Anterograde amnesia may be due to a deficit at any point along this processing chain. Retrograde amnesia, on the other hand, is presumed to reflect a problem with memory storage or retrieval mechanisms.

There is a broad consensus that major roles in the orchestration of encoding and retrieval of information necessary for declarative memory are played by the medial temporal lobes and prefrontal cortex, whilst the information itself is stored in other brains regions, predominantly posterior sensory and association cortex. The medial temporal lobes are often conceptualised as "indexing" and binding together 
information stored in these other brain regions, whilst the frontal lobes are critical for engaging the 'retrieval mode', performing a strategic search, response monitoring and inhibition of inappropriate information (Simons and Spiers, 2003; Wheeler et al., 1997).

\subsubsection{Memory consolidation}

The notion of consolidation is central to much theorising about long-term memory. The term was introduced in 1900 by Müller and Pilzecker (Müller and Pilzecker, 1900), who observed that new memories are initially fragile and may be disrupted by subsequently learned information, whereas older memories appear resistant to this 'retroactive interference'. Consolidation refers to the processes whereby a memory trace gradually becomes more stable over time. Shortly before Müller and Pilzecker introduced the term, the idea of memory consolidation had been proposed by Théodule Ribot based on clinical data. This French psychologist noted that patients with organic amnesia typically show loss of recently acquired memories but sparing of memories for the distant past. His observation that, in memory, "progressive destruction advances progressively from the unstable to the stable" (Ribot, 1881) has become known as Ribot's Law. The concept was developed further by Burnham, who maintained that: "There must be time for the processes of organization and assimilation (of memory) to take place. There must be time for nature to do her part ... Hurry defeats its own end" (Burnham, 1903).

Two principle applications of the word 'consolidation' in memory research can be distinguished (Dudai, 2004). Firstly, it is used to refer to the processes of protein synthesis and synaptic modification that occur in neurones during and in the seconds to hours following learning. This 'cellular' or 'early' consolidation has been extensively studied in a variety of non-humans animals, and particularly in the hippocampus of live rodents engaged in behavioural tasks. Secondly, 'consolidation' is used to refer to the long-term processes of neural reorganisation that underlie the temporal gradient of retrograde memory loss seen in organic amnesia and described by Ribot's Law. Although the existence of some sort of 'systems' or 'late' consolidation is widely accepted, the extent to which it applies to different types of 
memory, the time course over which it operates and the brain systems involved are the subject of considerable debate.

\subsubsection{The standard theory of systems consolidation}

According to what has become known as the 'standard theory' of consolidation (Bayley et al., 2005; Squire and Alvarez, 1995; Squire et al., 1993), long-term declarative memories are initially dependent upon both the hippocampal complex (hippocampus proper, dentate gyrus and subicular complex, entorhinal, perirhinal and parahippocampal cortices) and neocortical regions (see Figure 1.7).

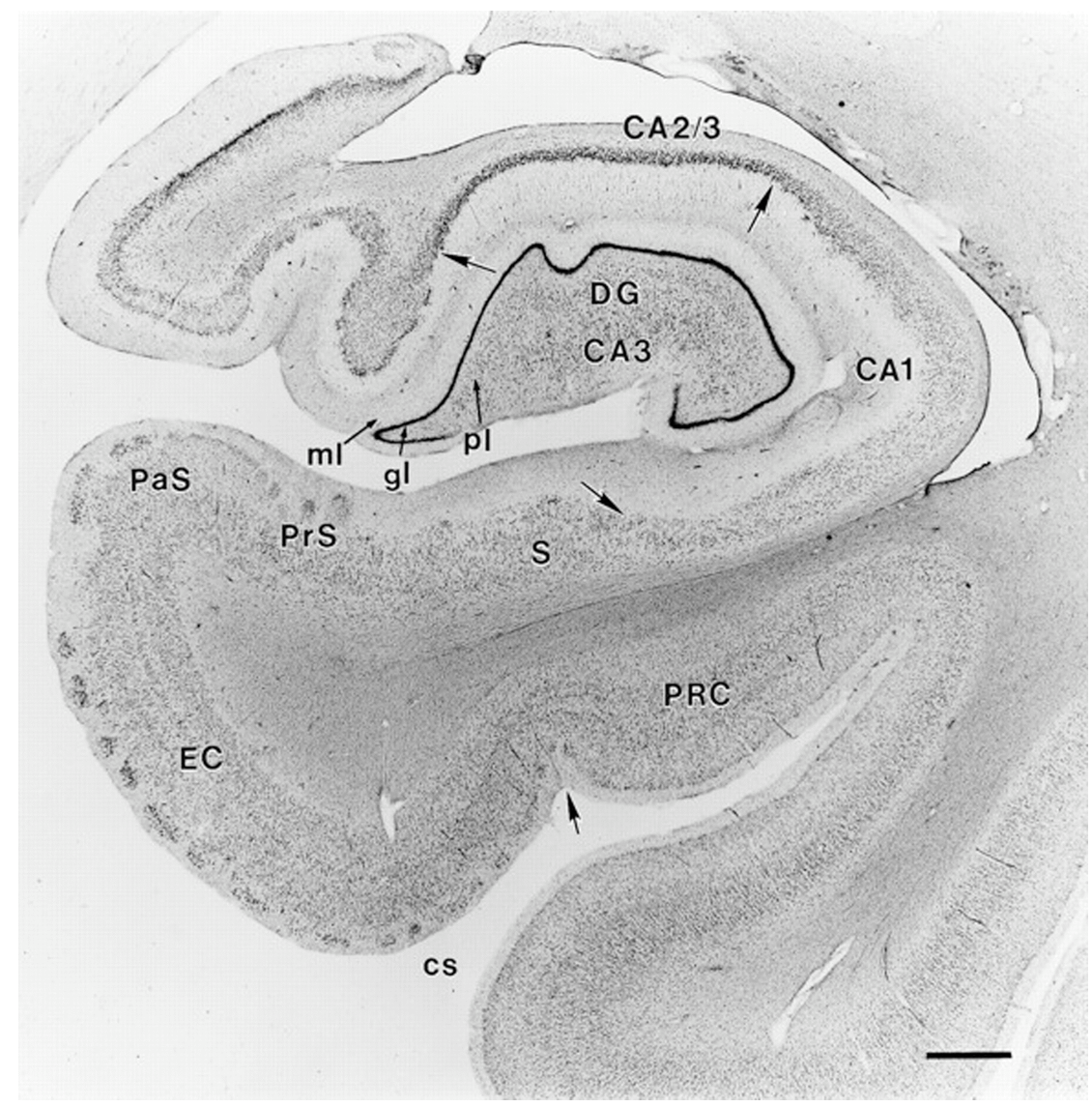

Figure 1.7: Coronal section from a normal human brain through the right rostral hippocampal formation stained with thionin. Arrows mark borders between regions. CA1 and CA2/3 refer to the CA fields of the hippocampus. DG, Dentate gyrus; $\mathrm{ml}$, molecular layer of the dentate gyrus; gl, granule cell layer; pl, polymorphic layer; $\mathrm{S}$, subiculum; PrS, presubiculum; PaS, parasubiculum; EC, entorhinal cortex; cs, collateral sulcus; PRC, perirhinal cortex. Scale bar, $1 \mathrm{~mm}$. 
Over a period of weeks or more, reorganisation of the trace renders it progressively less dependent on the hippocampal complex until it eventually becomes based entirely in the neocortex. The mechanisms underlying this trace reorganisation are not yet known. Several lines of evidence suggest that it requires reactivation of the memory trace, either in explicit rehearsal or implicit processing, and that this induces synaptic changes within the neocortical network (Squire, 1992). The standard theory does not draw clear distinctions between the functional roles of the different cortical regions within the medial temporal lobe and does not, therefore, predict dissociations in the type of declarative memory predominantly affected by damage to these different regions. Instead, the overall severity of declarative memory impairment is thought to be directly proportional to the extent of medial temporal damage (Zola-Morgan et al., 1994)

\subsubsection{The multiple trace theory}

Over the past decade, the standard theory of consolidation has faced a sustained challenge from proponents of the alternative 'multiple trace theory' (MTT) (Moscovitch and Nadel, 1998; Moscovitch et al., 2006; Nadel and Moscovitch, 1997). This theory is particularly concerned with 'episodic' memories - memories for unique, personally experienced events that are embedded in a specific spatial and temporal context, and whose recollection is associated with a feeling of reliving the past or 'autonoetic consciousness' (Tulving, 2002; Wheeler et al., 1997). The originators of the MTT were motivated by observations that, in some patients, highly focal hippocampal damage may result in a retrograde loss of episodic memories that extends back over decades or even across the entire lifespan. The MTT proposes that the hippocampus never relinquishes its role in the storage of an episodic memory trace. Furthermore, each time an episodic memory is retrieved, it is subsequently reencoded, leading to the establishment of multiple traces mediated by hippocampalneocortical neural ensembles. Older, often recollected memories will therefore be more likely to survive partial hippocampal damage, a consequence that could explain the temporal gradient seen in some amnesic patients. With regard to semantic memories, free of temporal and spatial specificity and not associated with autonoetic consciousness, the MTT is in accord with the standard theory: through as yet 
undefined processes of consolidation, semantic memory traces gradually become supported solely by the neocortex.

\subsubsection{Forgetting}

The long-term fate of declarative memories is of particular relevance to the study of TEA. In contrast to the classical amnesic syndrome, TEA patients do not exhibit an immediate inability to retain new information. Instead, newly acquired memories are reported to fade over days to weeks, the phenomenon of ALF.

There are longstanding and vexed questions about the mechanisms that underlie forgetting. Many of them originated in the late nineteenth century with the pioneering work of Hermann Ebbinghaus who published his monumental work Über das Gedächtnis (later translated into English as 'Memory: a contribution to experimental psychology') (Ebbinghaus, 1885) in 1885 whilst working at the University of Berlin. In this book, Ebbinghaus refers to heroic experiments that he conducted on himself and which involved the learning and recollection of large numbers of nonsense syllables. Most famously, he described the normal 'forgetting curve' - the exponential decay of newly acquired memories over a period of days. However, the notion of a passive process of memory decay is at best difficult to prove and at worst incoherent. In order to identify such a process, it would have to be observed in isolation from all factors that might actively cause forgetting save the passage of time. The most prominently discussed factor is interference from other stimuli and tasks encountered either before (proactive interference) or after (retroactive interference) the material to be remembered. The relevance of proactive interference outside the laboratory is questionable (Warrington and Weiskrantz, 1978). Retroactive interference, on the other hand, is likely to play a significant role in determining the rate at which new information is forgotten and recent work has shown that minimization of retroactive interference can significantly enhance delayed retrieval even in amnesic patients (Cowan et al., 2004; Della Sala et al., 2005). It has been proposed that cognitive activity occurring during the delay between learning and retrieval results in increased forgetting by interfering with normal memory consolidation (Dewar et al., 2007). In general, however, it is widely believed that, once information has been successfully encoded into long-term memory, forgetting occurs at a rate unaffected by 
experimental manipulation (Slamecka and McElree, 1983; Underwood, 1954), interindividual differences (Maylor, 1993) or neurological disease (Kopelman, 1985). The phenomenon of ALF challenges this assumption and offers a window onto processes of long-term memory consolidation.

\subsubsection{Autobiographical memory loss}

As described above, another prominent type of memory problem encountered by patients with TEA is a patchy loss of memories for remote autobiographical events, such as holidays or weddings. Autobiographical recollection, in particular the ability to mentally 'relive' certain past experiences, relies upon the complex integration of a wide range of cognitive processes including episodic and semantic memory, selfreflection, emotion, attention, visual imagery and executive function. Studies using functional neuroimaging implicate an extensive network of brain regions, including the medial and lateral temporal, prefrontal and retrosplenial/posterior cingulate cortices, the temporoparietal junction and the cerebellum, in the activation of autobiographical memories (Fink et al., 1996; Levine et al., 2004; Maguire, 2001; Svoboda et al., 2006). It is therefore unsurprising that pathology in a variety of brain regions can lead to autobiographical memory impairment, and that the nature of the impairment varies according to the brain region affected (Greenberg and Rubin, 2003). Medial temporal lobe damage can cause a temporally graded (Rempel-Clower et al., 1996) or extensive (Rosenbaum et al., 2004) retrograde amnesia for autobiographical event memories. On the other hand, damage to the frontal lobes tends to impair search strategies, resulting in apparently greater impairment of autobiographical memory recall than recognition (Kopelman and Stanhope, 1998), and difficulties with temporal ordering of memories across the lifespan (Shimamura et al., 1990). Frontal lobe damage is also associated with confabulation, thought to be due, at least in part, to an inability to inhibit the activation of inappropriate information (Metcalf, 2007). Some authorities have claimed that the disconnection of frontal and temporal regions involved in autobiographical memory function may lead to a specific impairment of 'ecphory' - that is, the interaction of retrieval cues with stored information - in autobiographical memory (Calabrese et al., 1996; Kroll et al., 1997). Autobiographical memory impairment has also been reported in cases of damage to the visual cortex - so-called visual memory-deficit amnesia (Ogden, 1993; 
Rubin and Greenberg, 1998). In this phenomenon, autobiographical memory, which is heavily dependent upon visual imagery, is impaired for events from all life periods prior to the onset of pathology. It has been argued (Rubin and Greenberg, 1998) that anterograde memory may be less markedly impaired in these cases because structures involved in encoding new material - the medial temporal lobes - are intact. Another brain region to which damage often results in autobiographical amnesia is the diencephalon. Korsakoff's syndrome is probably the most commonly studied example in this category, but the interpretation of its associated cognitive deficits is made problematic by the multiple complications of chronic alcohol abuse (Butters, 1985). Studies of patients with bilateral paramedian thalamic strokes have, however, typically revealed a profound and temporally extensive autobiographical amnesia alongside anterograde memory impairment (Graff-Radford et al., 1990; Hodges and McCarthy, 1993). This picture has been interpreted as resulting from a disconnection of anterior and posterior systems necessary for effective retrieval of episodic memories (Hodges and McCarthy, 1993).

Remote autobiographical memory loss in the context of apparently normal anterograde memory function, as described in cases of TEA, has been termed focal retrograde amnesia (FRA) and has been reported in association with a number of other neurological disorders (Kapur, 1993a), including head injury (Hunkin et al., 1995; Kapur et al., 1992; Levine et al., 1998), herpes simplex encephalitis (Fujii et al., 1999; O'Connor et al., 1992; Tanaka et al., 1999), cardiac arrest (Manning, 2002), stroke (Costello et al., 1998; Miller et al., 2001) and cerebral vasculitis (Evans et al., 1996; Evans et al., 2003). In comparison with lesions that result in focal anterograde memory deficits, the anatomical distribution of the brain pathology in cases of FRA is remarkably heterogeneous, and may be very diffuse (Evans et al., 1996; Evans et al., 2003) or more focally involve bilateral temporal (Fujii et al., 1999; Tanaka et al., 1999), frontal (Costello et al., 1998; Levine et al., 1998), posterior (Hunkin et al., 1995; Rubin and Greenberg, 1998) or subcortical (Miller et al., 2001) brain regions. In other cases, particularly those following mild head injury (De Renzi et al., 1995; Stracciari et al., 1994), no evident pathology is seen on neuroimaging. This diversity suggests that the underlying mechanisms of the amnesia may be similarly diverse. A number of hypotheses have been proposed including the failure of frontotemporal memory retrieval mechanisms as a result of organic (Levine et al., 1998) as well as 
psychological (Kopelman, 2000) pathology, recurrent, transient impairment of medial temporal lobe memory function (Kapur et al., 1989) and disrupted cortico-cortical connectivity (Evans et al., 2003).

Despite the large number of case reports in recent years, the notion of FRA has raised considerable controversy for several reasons (Kapur, 2000; Kopelman, 2000). In particular, it has been questioned whether anterograde memory is truly normal in any of the cases reported, and thus whether storage and retrieval mechanisms can really be considered intact for new memories but impaired for old memories. Kopelman (2000) points out that anterograde and retrograde memory are usually assessed using very different techniques and that, therefore, 'like' is not being compared with 'like'. Furthermore, he notes that, whilst standard anterograde memory testing may be normal in cases of apparent FRA, testing over extended delays may reveal accelerated long-term forgetting due to an inability to consolidate new memories into damaged long-term memory stores. In some cases, therefore, FRA might more appropriately be called disproportionate retrograde amnesia. A further reason for caution in interpreting cases of FRA is the observation that this pattern of memory impairment is similar to that usually found in cases of 'functional' or 'psychogenic' amnesia (Kopelman, 2002a; Markowitsch, 2003). In many cases of FRA, psychological mechanisms are difficult to rule out and may make the attribution of causality to organic pathology more tenuous. Finally, it has often been considered difficult to construct theories of how brain dysfunction can impair retrieval of certain kinds of memory that were acquired prior to brain injury, but leave the ability to acquire, retain and retrieve similar new memories relatively intact. Taking these factors into consideration, the apparently widespread complaints of autobiographical memory impairment but relatively normal anterograde memory performance among patients with TEA (Zeman et al., 1998) may offer a unique opportunity to investigate the phenomenon of FRA.

\subsection{Structure of the thesis}

The work described in this thesis is based upon a study of 50 patients with TEA from around the United Kingdom, the TIME (The Impairment of Memory in Epilepsy) Project. The clinical features of the TIME cases are reported in Chapter 2, providing 
an authoritative description of TEA for the first time. In Chapter 3, the TIME results are put into context by a comprehensive review of the clinical features of all reported cases of TEA. Chapter 4 focuses upon the neuropsychological features of TEA and includes a demonstration of ALF and autobiographical memory loss. In Chapter 5, I comprehensively review the existing literature on these two controversial forms of memory deficit. I highlight a number of important methodological challenges that may be encountered in their investigation, but conclude that they are demonstrable not only in TEA, but also in other forms of epilepsy. Chapter 6 reports the quantitative analysis of structural MRI data obtained from the TIME participants and their relationship with the neuropsychological findings. Chapter 7 is a detailed case report of a patient with TEA, whose journal entries provide remarkable insight into the condition and in whom the radiological investigations offer new and convincing evidence of the anatomical basis of the amnesic episodes. Finally, in Chapter 8, I draw together the findings of preceding chapters and suggest potentially fruitful avenues for further research. 
Chapter 1 


\section{CHAPTER TWO}

\section{The clinical characteristics of transient epileptic amnesia}

\subsection{Introduction}

The existing literature on transient epileptic amnesia (TEA) consists primarily of case reports and short case series. These reveal only an incomplete picture of the phenomenon. They suggest that TEA characteristically begins in later life, that amnesic attacks are relatively brief, recurrent and associated with waking and that anticonvulsant medication is usually effective in controlling seizures. In addition, patients often complain of unusual, persistent memory problems despite normal performance on standard neuropsychological tests. These previous reports are reviewed in Chapter 3. The aim of the work reported in this chapter was to perform a systematic study of TEA in a substantial, prospectively recruited group of patients, and thus to provide an authoritative account of the clinical features of the condition and explore its aetiology. The study, based in Edinburgh and conducted in collaboration with colleagues in Cambridge, is entitled the TIME (The Impairment of Memory in Epilepsy) Project.

TEA is assumed to be a relatively rare condition. A UK-wide recruitment process was therefore needed to identify a large number of patients in a realistic time frame. Furthermore, it was accepted that, although assessment for inclusion in the TIME Project would be prospective, the diagnosis of TEA would usually already have been made by the patient's physician, in some cases several years beforehand, and that treatment may already have been started. Assessment of the amnesic attacks was therefore based upon the patient and witness accounts and upon available clinical records. This was considered a reasonable approach, since it is the manner in which most diagnoses of epilepsy are made. To improve consistency in the recording of clinical information, a pro forma was used as a basis for history taking and each patient, together with someone who had witnessed his or her attacks, was interviewed by the same investigator. Using this method, information was gathered on: i) patient demographics; ii) the amnesic attacks: the patient's age at onset; the total number and 
average frequency; the typical features of the amnesic attacks, in particular, the time of day they typically occurred, the extent of retrograde and anterograde amnesia, their duration, and any additional features such as an aura, automatisms, brief losses of responsiveness or olfactory hallucinations; iii) any complaints of ongoing memory difficulties; iv) past medical history including risk factors for epilepsy, cerebrovascular disease and migraine; v) current and previous medication and vi) social and family history and systemic enquiry. Given potential concern regarding the contribution of psychogenic mechanisms to patients' memory complaints (see Chapter 1), particular attention was paid to past and current symptoms of mood disorder and any history of medically unexplained symptoms.

The cause of TEA is unknown. Of the 10 TEA patients studied by Zeman et al (1998), three had a history of myocardial infarction, one of recent aortic valve replacement and one of coronary bypass grafting. The authors hypothesised that hypoxic injury, which preferentially affects medial temporal regions, might result in a hippocampal seizure focus and subsequent amnesic seizures. This might also explain why TEA usually begins in later life. In the present study, the hypothesis that vascular disease plays an aetiological role in TEA was explored. The presence and extent of vascular disease was assessed through clinical history taking and examination, by eliciting the presence of vascular risk factors, and by neuroimaging.

Due to practical and financial constraints, EEG analysis was restricted to reviewing data originally obtained in the course of standard clinical investigations. A detailed neuropsychological evaluation and structural MRI brain scanning were performed on each patient. In this chapter, the clinical features of the patients in the TIME Project are described. The neuropsychological data are reported in Chapter 4 and the neuroimaging data in Chapter 6.

\subsection{Methods}

\subsubsection{Patient recruitment}

Cases of TEA (incident or established) were recruited to the TIME Project from around the United Kingdom via the Cognitive Disorders Clinics at the Western 
General Hospital, Edinburgh and at Addenbrooke's Hospital, Cambridge and via the British Neurological Surveillance Unit (BNSU) - a service of the Association of British Neurologists. The BNSU facilitates recruitment of rare neurological conditions by regularly alerting UK neurologists to current research projects and providing a channel for case notification. Following notification of a case, clinic letters and investigation results were reviewed. Patients likely to meet inclusion criteria were interviewed either at their own home or, if convenient, at the Wellcome Trust Clinical Research Facility at the Western General Hospital, Edinburgh.

For inclusion in the TIME Project, patients needed to fulfil the following diagnostic criteria adapted from Zeman et al. (1998):

1) a history of recurrent witnessed episodes of transient amnesia

2) cognitive functions other than memory judged to be intact during typical episodes by a reliable witness

3) evidence for a diagnosis of epilepsy based on one or more of the following:

a. epileptiform abnormalities on electroencephalography

b. the concurrent onset of other clinical features of epilepsy (e.g. lipsmacking, olfactory hallucinations)

c. a clear-cut response to anticonvulsant therapy

The study was approved by the Multi-Centre Research Ethics Committee, United Kingdom (MREC 03/10/77). All participants gave written, informed consent.

\subsubsection{Clinical characteristics of TEA}

All interviews were conducted by a single investigator (CRB). In each case, a detailed history was obtained from the patient and at least one witness. A pro forma (see Appendix A) was used to collect data on demographics; clinical features of the amnesic attacks; other transient neurological or cognitive symptoms; interictal memory complaints; risk factors for epilepsy (birth injury, febrile seizures, significant head injury (leading to a loss of consciousness), intracranial infection, alcohol abuse, recreational drugs, family history) and cerebrovascular disease (see below); past medical and psychiatric history. Major depression, generalised anxiety disorder and panic disorder subsections of the Structured Clinical Interview for Diagnostic and 
Statistical Manual of Mental Disorders, Fourth Edition (DSM-IV) (First et al., 2002) were administered. A neurological examination was performed. Medical case notes and correspondence were reviewed.

\subsubsection{Electroencephalography}

EEG was performed in 49 cases as part of routine clinical care prior to the patients' entry into the TIME Project. The type of recording used - routine, sleep-deprived or ambulatory - was therefore variable. The traces were reviewed by consultant neurophysiologists at the referring centres rather than a single investigator. Although requests were made for copies of the EEG traces, many data sets were unobtainable. Results were therefore based upon the clinical reports and were classified as: 1) normal or as showing 2) non-specific or 3) clear, recurrent epileptiform abnormalities, either bilaterally or over the left or right hemisphere.

\subsubsection{Cerebrovascular disease risk factors and TEA}

Using a series of standard questions, data were collected from participants regarding: 1) previous events potentially associated with cerebral hypoxia including cardiac arrythmias, cardiac valve disease, myocardial infarction or other possible hypotensive episodes and 2) cerebrovascular disease risk factors (CVRFs) comprising hypertension, diabetes mellitus, smoking, previous TIA or stroke, ischaemic heart disease or peripheral vascular disease and any family history of stroke, ischaemic heart disease or peripheral vascular disease.

Control data were made available from the Lothian Birth Cohort 1936 Study (LBC1936). This ongoing study, run by the University of Edinburgh Department of Psychology, collects clinical and neuropsychological data from people who were born in 1936 and underwent cognitive testing at the age of 11 as part of the "Scottish Mental Survey". This control dataset was chosen because of its large size and mean age of 67 years, close to that of the TIME patients. In clinical studies, CVRFs tend to have a relatively small effect on the outcomes of interest so having a large sample size is critical. For the purposes of the TIME Project, 434 participant records from 
LBC1936 were examined and information about CVRFs were extracted using a pro forma identical to that used for TEA patients ${ }^{1}$.

\subsubsection{Statistics}

Descriptive statistics, odds ratios and comparisons of proportions were performed using SPSS for Windows 15.0. Chi-squared tests or, where necessary, Fisher's exact test were used to calculate the significance of observed associations between group (patient or control) and clinical findings.

\subsection{Results}

\subsubsection{Clinical characteristics of TEA}

A total of 50 patients (34 males) fulfilling the diagnostic criteria for TEA were recruited between August 2003 and April 2005. During this period, a total of 109 referrals were received, 40 of which did not meet the study criteria and 19 of which arrived after the target 50 subjects had been recruited. Of the 40 excluded referrals, 37 were excluded following a review of the medical records and telephone conversations with the patients and witnesses, and three were excluded after a full interview. Sixteen did not have reliable witness evidence of any episodes during which cognitive functions other than memory was intact, 11 had recurrent amnesic attacks but no convincing evidence for a diagnosis of epilepsy, six did not respond to the invitation to take part in TIME, four refused consent, two had only a single amnesic episode and one died before enrolment. Illustrative examples of excluded cases are given in Appendix D. Of the 50 patients included in the TIME Project, 30 were diagnosed and referred during the study period, 10 already carried the diagnosis and were referred from other hospitals and 10 had been previously diagnosed at one of the two study centres. The geographical spread of referrals is illustrated in Figure 2.2.

\footnotetext{
${ }^{1}$ The data extraction from LBC1936 was performed under my supervision by a fourth-year medical student, Justin Penge, for his Special Study Component (SSC4) research project.
} 


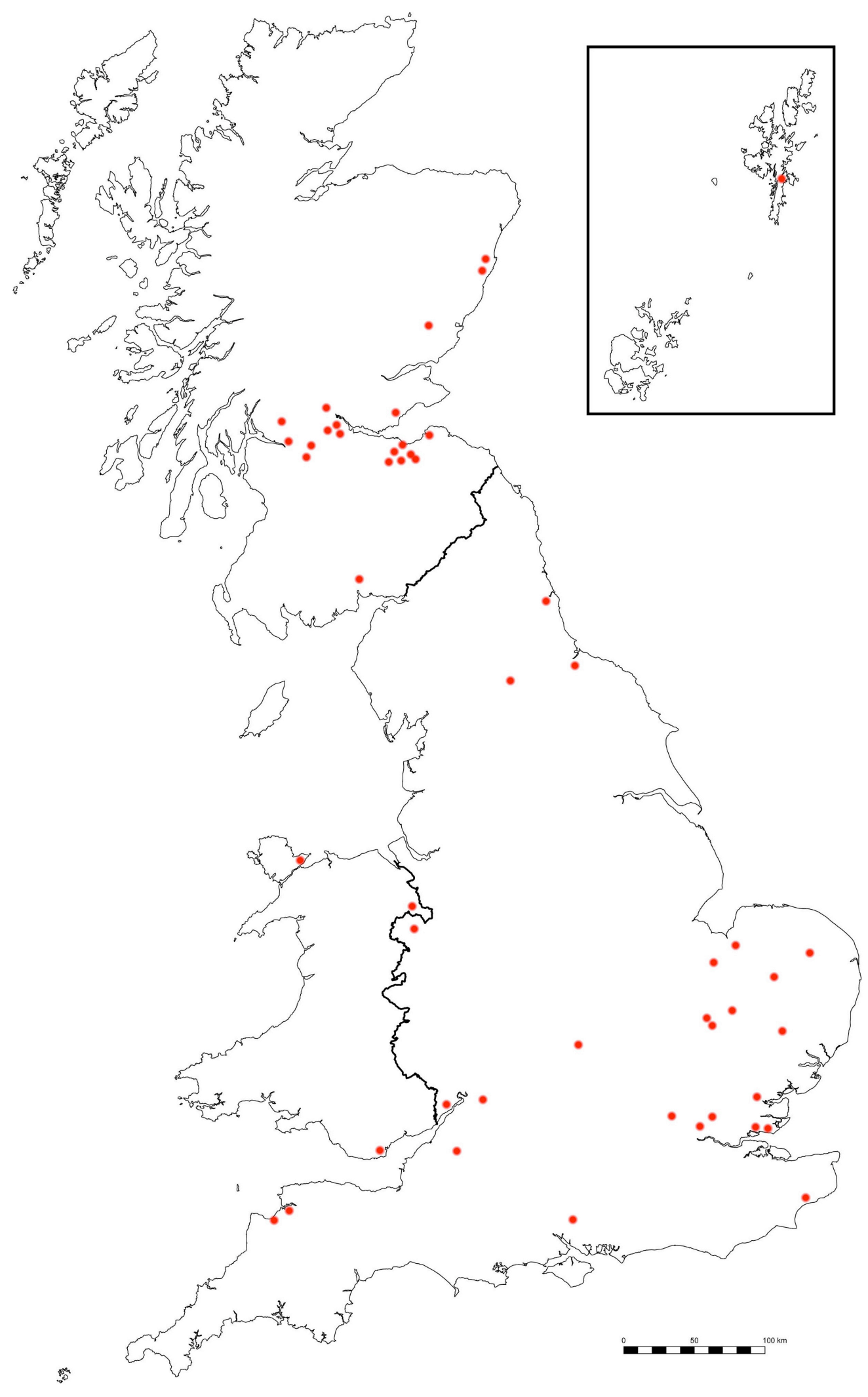

Figure 2.2: The geographic spread of TEA referrals included in the TIME Project. Each patient is represented by a red dot. 
The distribution of patients meeting each of the three diagnostic criteria for epilepsy used in this study is shown in Table 2.1 and Figure 2.3. The cases are ordered by the number of diagnostic criteria met (descending) and then by the total number of attacks reported (ascending).

\begin{tabular}{|c|c|c|c|c|c|c|c|c|c|c|}
\hline $\begin{array}{l}\text { ID } \\
\text { number }\end{array}$ & Sex & $\begin{array}{c}\text { Age at } \\
\text { onset } \\
\text { (years) }\end{array}$ & $\begin{array}{c}\text { Total no. } \\
\text { amnestic } \\
\text { attacks }\end{array}$ & $\begin{array}{l}\text { First to last } \\
\text { attack } \\
\text { (months) }\end{array}$ & $\begin{array}{l}\text { Duration of } \\
\text { attacks }\end{array}$ & $\begin{array}{l}\text { Amnesia } \\
\text { upon waking }\end{array}$ & $\begin{array}{l}\text { No. } \\
\text { criteria } \\
\text { met }\end{array}$ & EEG & $\begin{array}{l}\text { Other features } \\
\text { sometimes present }\end{array}$ & $\begin{array}{l}\text { Treatment } \\
\text { response }\end{array}$ \\
\hline 11 & $\mathrm{~F}$ & 57 & 5 & 4 & $5-15 \mathrm{~min}$ & yes & 3 & epil & autom & com \\
\hline 56 & $\mathrm{~F}$ & 49 & 5 & 72 & $2-24 \mathrm{hr}$ & yes & 3 & epil & olf hall & com \\
\hline 112 & M & 54 & 6 & 12 & $30 \mathrm{~min}-1 \mathrm{hr}$ & yes & 3 & epil & autom/unresp & com \\
\hline 37 & M & 61 & 6 & 2 & $15-30 \mathrm{~min}$ & yes & 3 & epil & olf hall/autom/CPS & com \\
\hline 49 & $\mathrm{~F}$ & 69 & 15 & 24 & $2-24 \mathrm{hr}$ & exclusively & 3 & epil & olf hall/autom/GTC & com \\
\hline 31 & $\mathrm{~F}$ & 57 & 15 & 17 & $<1 \mathrm{~min}$ & & 3 & epil & olf hall/unresp & com \\
\hline 83 & M & 72 & 18 & 21 & $15-30 \mathrm{~min}$ & yes & 3 & epil & olf hall/autom & com \\
\hline 42 & $\mathrm{~F}$ & 70 & 50 & 86 & $2-24 \mathrm{hr}$ & exclusively & 3 & epil & GTC & partial \\
\hline 8 & $\mathrm{~F}$ & 67 & 50 & 18 & $5-15 \mathrm{~min}$ & yes & 3 & epil & olf hall & com \\
\hline 21 & M & 53 & 60 & 156 & $2-24 \mathrm{hr}$ & yes & 3 & epil & olf hall & com \\
\hline 12 & $\mathrm{~F}$ & 63 & 2 & 5 & $30 \mathrm{~min}-1 \mathrm{hr}$ & exclusively & 2 & & olf hall & com \\
\hline 61 & M & 71 & 2 & 3 & $2-24 \mathrm{hr}$ & exclusively & 2 & & olf hall & com \\
\hline 22 & M & 58 & 3 & 3 & $30 \mathrm{~min}-1 \mathrm{hr}$ & exclusively & 2 & & olf hall & com \\
\hline 116 & M & 44 & 3 & 283 & 4 days & & 2 & epil & (pure amnesia) & com \\
\hline 40 & M & 65 & 4 & 12 & $2-24 \mathrm{hr}$ & yes & 2 & & CPS & com \\
\hline 64 & M & 60 & 4 & 6 & $5-15 \min$ & & 2 & & olf hall & com \\
\hline 94 & M & 69 & 5 & 4 & $30 \mathrm{~min}-1 \mathrm{hr}$ & yes & 2 & & autom & com \\
\hline 38 & $\mathrm{~F}$ & 45 & 5 & 7 & 15-30min & yes & 2 & & olf hall & com \\
\hline 93 & M & 60 & 6 & 6 & $2-24 \mathrm{hr}$ & yes & 2 & & autom/unresp & com \\
\hline 95 & $\mathrm{~F}$ & 52 & 6 & 168 & $2-24 \mathrm{hr}$ & yes & 2 & epil & (pure amnesia) & com \\
\hline 55 & M & 59 & 7 & 6 & $30 \mathrm{~min}-1 \mathrm{hr}$ & & 2 & & olf hall/autom & com \\
\hline 3 & M & 70 & 8 & 4 & 15-30min & & 2 & & autom/unresp & com \\
\hline 62 & M & 56 & 8 & 16 & $30 \mathrm{~min}-1 \mathrm{hr}$ & exclusively & 2 & & olf hall & com \\
\hline 50 & M & 77 & 10 & 27 & 2 days & yes & 2 & & autom & com \\
\hline 68 & M & 68 & 10 & 12 & $15-30 \mathrm{~min}$ & & 2 & & olf hall & com \\
\hline 88 & M & 49 & 10 & 4 & $15-30 \mathrm{~min}$ & exclusively & 2 & epil & (pure amnesia) & com \\
\hline 13 & M & 75 & 12 & 16 & 15-30min & yes & 2 & & olf hall & com \\
\hline 91 & M & 69 & 15 & 18 & $15-30 \mathrm{~min}$ & yes & 2 & epil & autom/unresp & not treated \\
\hline 36 & M & 76 & 16 & 9 & $15-30 \mathrm{~min}$ & exclusively & 2 & & olf hall & com \\
\hline 69 & M & 59 & 16 & 5 & $15-30 \mathrm{~min}$ & yes & 2 & & unresp & com \\
\hline 32 & M & 71 & 19 & 7 & $15-30 \mathrm{~min}$ & & 2 & & autom & com \\
\hline 9 & M & 52 & 20 & 24 & 15-30min & yes & 2 & & autom/unresp & com \\
\hline 114 & $\mathrm{~F}$ & 61 & 25 & 65 & $2-24 \mathrm{hr}$ & yes & 2 & & autom/unresp & com \\
\hline 39 & $\mathrm{~F}$ & 50 & 30 & 67 & $5-15 \min$ & & 2 & & autom/unresp & com \\
\hline 82 & $M$ & 65 & 30 & 33 & $15-30 \mathrm{~min}$ & yes & 2 & & olf hall/autom & com \\
\hline 47 & M & 60 & 30 & 72 & $2-24 \mathrm{hr}$ & & 2 & & olf hall/unresp & com \\
\hline 138 & $\mathrm{~F}$ & 59 & 30 & 130 & $2-24 \mathrm{hr}$ & yes & 2 & epil & (pure amnesia) & com \\
\hline 10 & $\mathrm{~F}$ & 66 & 36 & 25 & $15-30 \mathrm{~min}$ & yes & 2 & epil & (pure amnesia) & com \\
\hline 90 & M & 60 & 50 & 40 & $30 \mathrm{~min}-1 \mathrm{hr}$ & exclusively & 2 & & olf hall & com \\
\hline 100 & $M$ & 48 & 50 & 55 & $30 \mathrm{~min}-1 \mathrm{hr}$ & yes & 2 & & olf hall/autom & $\mathrm{com}$ \\
\hline 16 & $\mathrm{~F}$ & 74 & 60 & 60 & $30 \mathrm{~min}-1 \mathrm{hr}$ & & 2 & & autom & partial \\
\hline 124 & $\mathrm{~F}$ & 52 & 60 & 144 & 15-30min & & 2 & epil & (pure amnesia) & com \\
\hline 73 & M & 71 & 3 & 9 & $30 \mathrm{~min}-1 \mathrm{hr}$ & yes & 1 & epil & (pure amnesia) & not treated \\
\hline 35 & M & 75 & 6 & 59 & 15-30min & yes & 1 & & (pure amnesia) & com \\
\hline 41 & $\mathrm{~F}$ & 65 & 6 & 9 & $1 \mathrm{hr}-2 \mathrm{hr}$ & & 1 & & (pure amnesia) & com \\
\hline 51 & M & 49 & 6 & 9 & $15-30 \mathrm{~min}$ & yes & 1 & & (pure amnesia) & com \\
\hline 92 & M & 73 & 6 & 6 & $1 \mathrm{hr}-2 \mathrm{hr}$ & yes & 1 & & (pure amnesia) & com \\
\hline 103 & M & 71 & 7 & 20 & $15-30 \mathrm{~min}$ & & 1 & & (pure amnesia) & com \\
\hline 25 & M & 75 & 15 & 46 & $30 \mathrm{~min}-1 \mathrm{hr}$ & exclusively & 1 & & (pure amnesia) & com \\
\hline 28 & M & 55 & 28 & 21 & $5-15 \min$ & exclusively & 1 & & (pure amnesia) & com \\
\hline
\end{tabular}




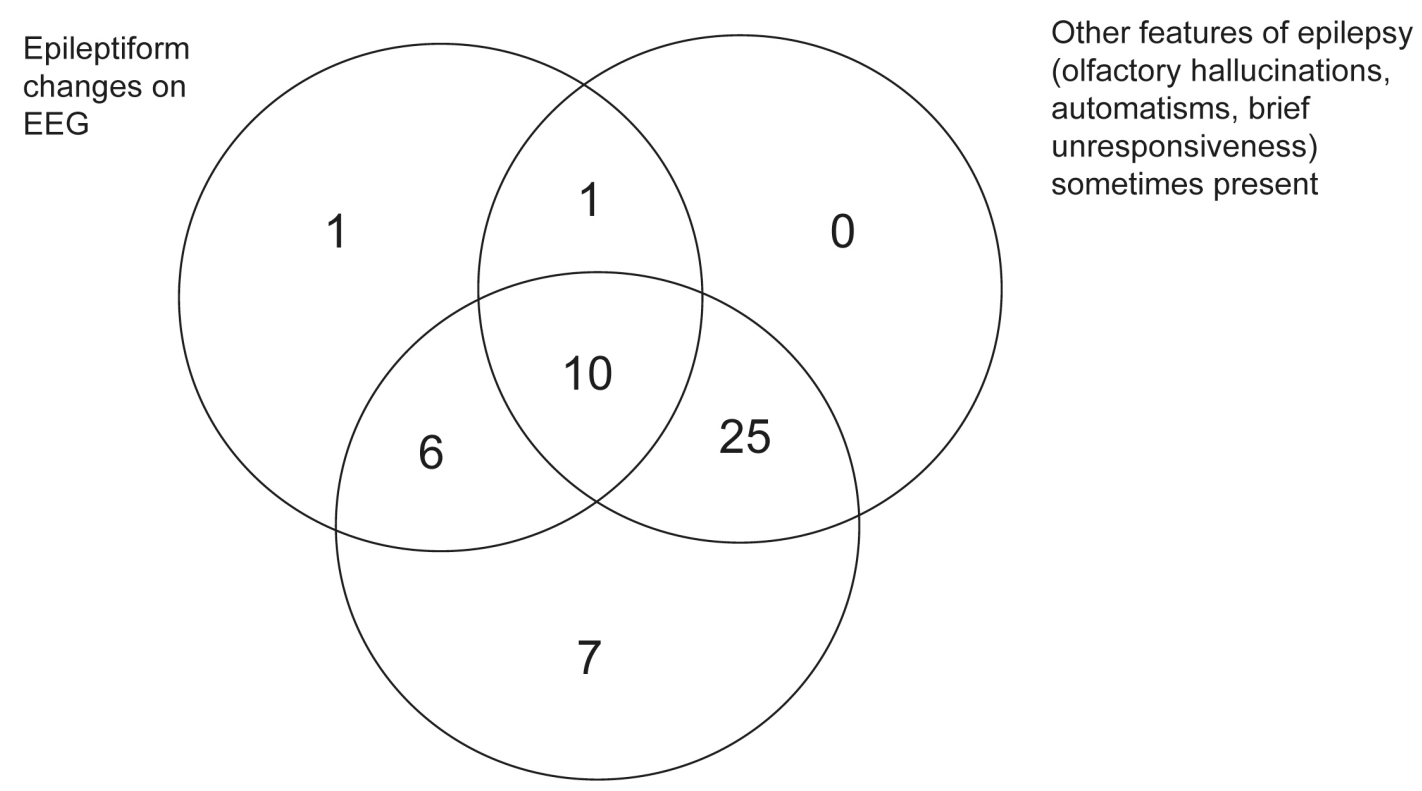

Clear-cut response to anticonvulsant medication

Figure 2.3: Venn diagram showing the distribution of patients meeting the three diagnostic criteria for epilepsy used in this study. EEG = electroencephalogram.

The mean age at the onset of amnesic attacks was 62.1 years (standard deviation (SD) 9.1; range 44 to 77 years), and at entry into the study was 68.3 years (SD 8.6; range 46 to 84 years). The mean duration of education was 12.2 years (SD 2.9).

All patients were referred to the TIME Project with a diagnosis of epilepsy. Initially, however, the following diagnoses had been entertained by the patient's physician: unspecified (26\%), temporal lobe epilepsy (24\%), transient global amnesia (20\%), 'psychogenic' (18\%), transient cerebral ischemia/stroke (6\%), cardiac arrhythmia (4\%), dementia (2\%). The median delay to the diagnosis of TEA was 12 months (mean 21 months, interquartile range (IQR) 5 to 25).

The clinical features are summarised in Table 2.2. A median total number of 10 amnesic attacks (IQR 6 to 30) was experienced over a median (first to last seizure) of 
17.5 months (IQR 6 to 56) at a median frequency of 12 attacks per year (IQR 5 to 20). Attacks occurred upon waking in 37 patients (74\%), exclusively so in 11 of these patients $(22 \%)$. No other consistent triggers were identified. Ten patients $(20 \%)$ described experiencing nonspecific symptoms, such as "a floaty feeling" or nausea, before the amnesia. Attack onset was sudden and heralded by questions betraying a loss of memory such as "Where am I?" "What are we doing here?" or "What day is it?" Twenty-five patients (50\%) typically exhibited repetitive questioning. Execution of complex, purposeful actions (see Box 2.1 and Appendix $C$ for examples), together with appropriate responses in conversation, were taken to indicate preservation of non-memory cognitive functions during the attack. Anterograde amnesia was often incomplete, with 28 patients (56\%) reporting at least partial recall of their attacks. The median duration of the amnesic attacks was '30 to 60 minutes' (range ' $<1$ minute' to 'days'), and $70 \%$ of patients typically experienced episodes lasting less than one hour.

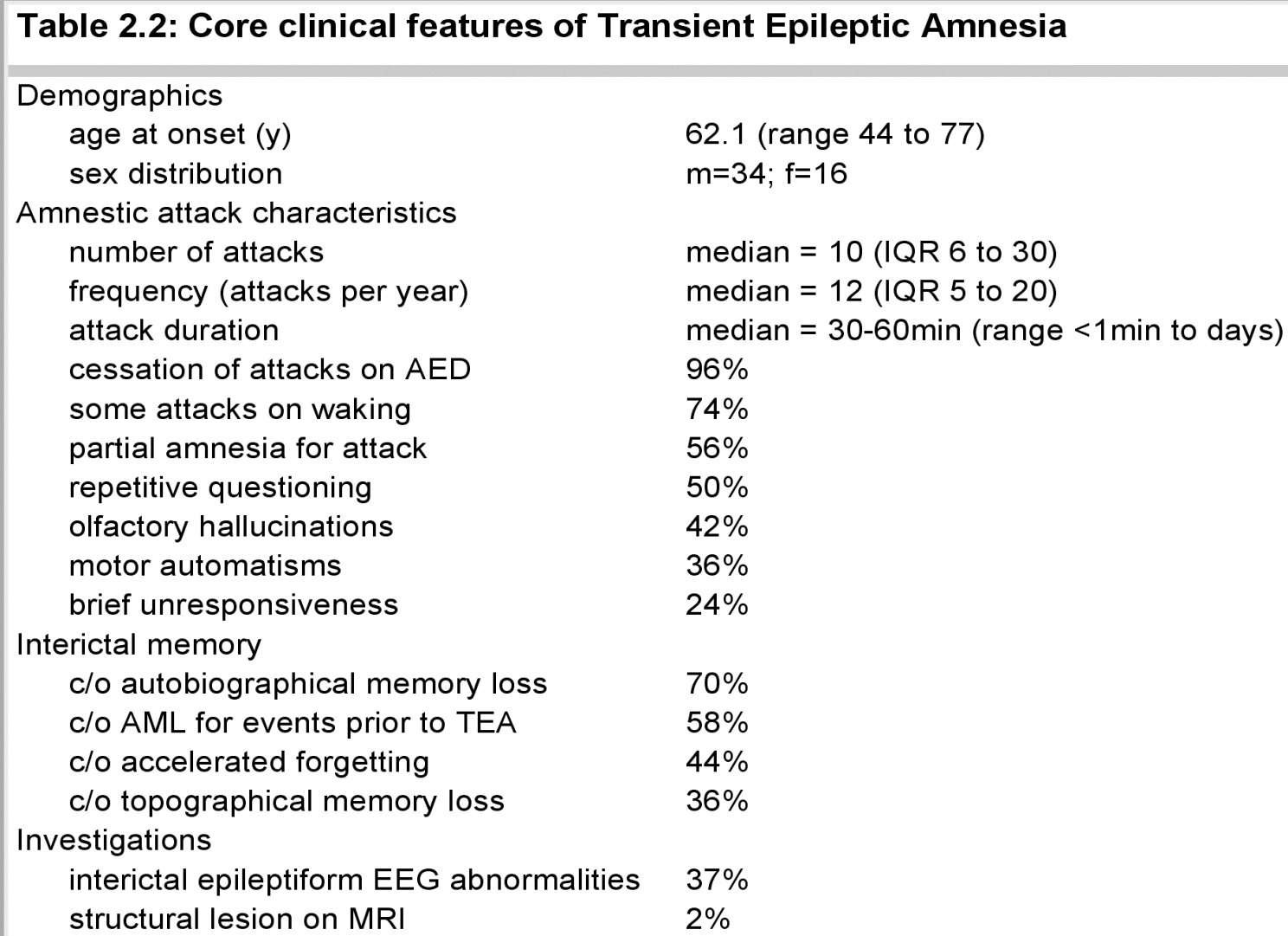

$A E D=$ antiepileptic drug; $A M L=$ autobiographical memory loss; $c / 0=$ complaint of; $I Q R=$ interquartile range 
Amnesia was the sole feature of all attacks in 14 patients (28\%), and 46 patients (92\%) experienced at least one such episode. However, certain other features sometimes preceded or accompanied the amnesia: olfactory or gustatory hallucinations (42\%); automatisms such as chewing, lip-smacking or fiddling with clothing (36\%); and brief episodes of unresponsiveness (24\%). Two patients suffered temporally distinct generalised tonic-clonic seizures.

Reports of persistent memory difficulties were common. Thirty-five patients (70\%) described a patchy loss of remote autobiographical memories. In 29 of these patients $(83 \%)$, some of the 'lost memories' had been acquired prior to the clinical onset of epilepsy, sometimes several decades before. Twenty-two patients (44\%) reported problems with "holding on to a memory for more than a few days", suggesting the presence of accelerated forgetting. Eighteen patients (36\%) had developed significant navigational difficulties.

A history of psychiatric disease was present in 12 patients (24\%) and five control subjects (21\%). The diagnoses comprised current major depression (two patients), previous depression (nine patients, four controls), past anxiety (one patient), past panic disorder (one control subject). No patient or control subject had a history of medically unexplained symptoms. 'Pathological emotionalism', usually inappropriate crying, was described by nine patients (18\%) and no controls. Eleven patients $(22 \%)$ and five control subjects (21\%) had experienced migraine at some time. Risk factors for epilepsy were minimally represented in patient and control groups. The frequencies of each were: birth trauma - one patient, one control; febrile seizures one patient, no controls; significant head injury - three patients, no controls; intracranial infection - no patients, no controls; and family history of epilepsy - no patients, one control. A history of alcohol consumption in excess of recommended levels (21 units/week for men, 14 units/week for women) was present in 12 patients (24\%) and four control subjects (17\%). Neurological examination did not demonstrate any major or consistent abnormalities. 


\section{Box 2.1: Three case reports that demonstrate common features of TEA}

\section{Case 1 (ID number 28)}

A 58-year old carpet fitter experienced 28 episodes of transient amnesia over 18 months. All occurred upon waking in the night and lasted about 20 minutes. He repetitively questioned his wife, but was responsive and coherent throughout. During one attack he was unable to recall the death of his brother a few days earlier. Routine EEG and MRI were normal. Lamotrigine abolished the attacks but they briefly returned, with associated olfactory hallucinations, during a period of non-compliance, and ceased again when he restarted the medication. At interview, he described rapid forgetting of recently acquired memories, patchy loss of salient autobiographical memories from the past 30 years, such as his wife's abdominal surgery and the wedding of his son, and significant new difficulties navigating around his local area.

\section{Case 2 (ID number 10)}

A 69-year old retired teacher of French had 23 episodes of transient memory loss over two years. All occurred late in the evening, were heralded by a "woozy feeling", and were characterized by loss of memory for events of the past two or three days and repetitive questioning. During attacks, she retained the ability to sight-read piano pieces, translate from French to English and perform mental arithmetic. Thirty minutes later, when the amnesia resolved, she was able to remember and record in her diary many details of the episode. MRI of the brain was normal but EEG revealed epileptiform abnormalities bilaterally in the temporal lobes. The attacks ceased on Carbamazepine. However, she complained of persistent difficulties recalling certain events from the distant past, including her daughter being badly kicked by a horse 35 years earlier and a holiday to Kenya four years earlier.

\section{Case 3 (ID number 112)}

A 55-year old mathematics teacher had only the vaguest recollection of his six witnessed episodes of amnesia, three of which occurred upon waking and one whilst he was driving. On each occasion, he would say to his wife "I've lost it. Where am I? What day is it?" and tried to "piece his memory back together" over about 30 minutes whilst continuing with other activities. MRI brain scan was normal. EEG revealed epileptiform abnormalities over the left hemisphere and the attacks ceased on Lamotrigine. He described a dense but patchy loss of autobiographical memories from the past 25 years, including his first holiday abroad in 1980, his daughter's wedding in 1999 and his silver wedding anniversary in 2003. He had also developed pronounced difficulties with route-finding, even in familiar environments. 
A total of 48 patients were taking anticonvulsant medication at the time of interview. In $46(96 \%)$ this had completely abolished the amnesic attacks. The remaining two patients had markedly reduced attack frequency. All patients were receiving monotherapy at low-to-moderate doses. Two patients had declined anticonvulsant medication.

\subsubsection{Electroencephalography}

A total of 49 patients (98\%) had undergone EEG. Clear, recurrent epileptiform abnormalities were present in 18 (37\%) patients (although only after sleep-deprivation in eight patients) and were consistently over temporal or frontotemporal regions. Eight were left-sided, six were right-sided and four were bilateral. Sixteen patients (32\%) had non-specific, focal slow wave changes and no abnormality was detected in 15 patients $(31 \%)$.

\subsubsection{Cerebrovascular disease risk factors and TEA}

The cerebrovascular risk factor data are shown in Table 2.3. The patient and control groups were well matched for age although the variance differed (patients: mean $=$ 68.3 years $(\mathrm{SD}=8.6)$, controls: mean $=67.0$ years $(\mathrm{SD}=1.1), \mathrm{p}=0.320)$. The proportion of males was greater in the patient group (odds ratio $(\mathrm{OR})=2.08,95 \%$ confidence interval $(\mathrm{CI})=1.11$ to $3.90, \mathrm{p}=0.02)$. The CVRFs significantly overrepresented in the patient group were: cardiac arrhythmias $(\mathrm{OR}=4.62, \mathrm{CI}=1.63$ to $13.12, \mathrm{p}=0.008)$, cardiac valve disease $(\mathrm{OR}=4.09, \mathrm{CI}=1.18$ to $14.10, \mathrm{p}=0.038)$ and arterial aneurysm $(\mathrm{OR}=15.96, \mathrm{CI}=1.4$ to $179.31, \mathrm{p}=0.003)$. Cigarette smoking was underrepresented in the patient group $(\mathrm{OR}=0.32, \mathrm{CI}=0.20$ to $0.65, \mathrm{p}<0.001)$. Of the six TEA patients with a history of cardiac arrhythmia, four had paroxysmal atrial fibrillation and two had been diagnosed with intermittent complete heart block following collapses and fitted with a permanent pacemaker. Two patients and one control subject had undergone elective repair of an abdominal aortic aneurysm. 


\begin{tabular}{|c|c|c|c|c|c|}
\hline & $\begin{array}{c}\text { Patients } \\
(n=50)\end{array}$ & $\begin{array}{c}\text { Controls } \\
(n=434)\end{array}$ & $\begin{array}{l}\text { Odds } \\
\text { ratio }\end{array}$ & $\begin{array}{c}95 \% \\
\text { confidence } \\
\text { interval }\end{array}$ & $\mathbf{p}$ \\
\hline mean age (SD) (yrs) & $67.0(8.7)$ & $67.0(1.1)$ & $\mathrm{n} / \mathrm{a}$ & $\mathrm{n} / \mathrm{a}$ & $\mathrm{n} / \mathrm{a}$ \\
\hline $\operatorname{sex}(M / F)$ & $34 / 16$ & $194 / 190$ & 2.08 & $1.11-3.90$ & 0.020 \\
\hline previous TIA/stroke & $3(6 \%)$ & $13(3 \%)$ & 1.82 & $0.50-6.63$ & $0.276^{*}$ \\
\hline hypertension & $23(46 \%)$ & $135(31 \%)$ & 1.57 & $0.87-2.85$ & 0.134 \\
\hline diabetes mellius & $2(4 \%)$ & $33(8 \%)$ & 0.43 & $0.13-1.91$ & $0.204^{*}$ \\
\hline IHD (MI or angina) & $6(12 \%)$ & $54(12 \%)$ & 0.83 & $0.34-2.05$ & 0.691 \\
\hline PVD & $1(2 \%)$ & $4(1 \%)$ & 1.94 & $0.21-17.70$ & $0.459^{*}$ \\
\hline cardiac arrhythmia & $6(12 \%)$ & $11(3 \%)$ & 4.62 & $1.63-13.12$ & $0.008^{*}$ \\
\hline cardiac valve disease & $4(8 \%)$ & $8(2 \%)$ & 4.09 & $1.18-14.10$ & $0.038^{*}$ \\
\hline arterial aneurysm & $2(4 \%)$ & $1(<1 \%)$ & 15.96 & $1.4-179.31$ & $0.003^{*}$ \\
\hline cigarette smoking (ever) & $13(26 \%)$ & $202(47 \%)$ & 0.32 & $0.20-0.65$ & $<0.001$ \\
\hline alcohol excess & $9(18 \%)$ & $62(14 \%)$ & 1.14 & $0.53-2.47$ & 0.739 \\
\hline FHx of TIA/stroke/IHD/PVD & $17(34 \%)$ & $157(36 \%)$ & 0.75 & $0.40-1.38$ & 0.35 \\
\hline
\end{tabular}

\subsection{Discussion}

In this chapter, I report the clinical findings of much the largest study of TEA to date. TEA typically begins in late-middle to old age: no patient in this study was below the age of 40 at the onset of amnesic attacks. In this series, the male to female ratio was $2: 1$. The amnesic attacks are characterised by a mixed anterograde and retrograde amnesia, sometimes with repetitive questioning. The anterograde component, however, is often incomplete and patients may report being able to "remember not being able to remember". Attacks commonly occur on waking, a helpful diagnostic clue. Almost all patients have some attacks of which amnesia is the only feature, but other episodes are accompanied by olfactory hallucinations, automatisms or a brief loss of responsiveness. The duration of the amnesic episodes is usually less than one hour but can be longer. The EEG shows epileptiform abnormalities in approximately one-third of cases. In common with other forms of late-onset epilepsy (Stephen and 
Brodie, 2000), TEA is responsive to relatively low doses of anticonvulsant medication. However, many patients complain of persistent memory difficulties after the cessation of attacks. Detailed discussion of these characteristic clinical features is postponed until Chapter 3, in which the TIME results are combined with a comprehensive review of the literature. In the remainder of this section, I discuss the diagnostic criteria, epidemiology, aetiology and, briefly, the pathophysiology of TEA.

The diagnosis of epilepsy as the cause of recurrent amnesic episodes is often not considered by clinicians. The evidence is most persuasive in those patients with support from all three criteria used in this study: 1) epileptiform changes on the EEG, 2) other features of epilepsy such as olfactory hallucinations, automatisms or a brief period of unresponsiveness and 3) a clear-cut response to anticonvulsant medication. Ten patients in the TIME Project satisfied all three criteria, a further 38 satisfied two and eight cases met only one criterion. This last group, in whom the diagnosis was least certain, was included on the basis that the clinical features were otherwise identical to those in the other two groups and were likely to reflect a common underlying mechanism. Treatment responsiveness is difficult to assess when attacks are infrequent. Five patients experienced fewer than four amnesic attacks, yet met the diagnostic criteria as follows. Cases 12, 22 and 63 had frequent olfactory hallucinations, which disappeared with anticonvulsant medication. Case 73 had frequent spikes over the left temporal region on routine EEG. All had brief episodes of amnesia upon waking associated with reports of interictal accelerated forgetting and autobiographical amnesia. Case 116 had three prolonged amnesic episodes, with clear right frontotemporal spikes on sleep EEG together with typical interictal memory complaints. Cases with typical clinical features, but for whom clear evidence of epilepsy was missing, were excluded (see Appendix D, case B1 for an example). Other possible causes of transient amnesia, including transient ischaemic attacks, migraine or psychiatric factors should always be carefully considered in cases of recurrent transient amnesia, especially when features atypical of TEA are present.

In all the cases described here, transient amnesia was the most prominent feature of the episodes and provided the patient's principle reason for seeking medical attention. The study deliberately included both patients with consistently 'pure' attacks of TEA, in which amnesia was the only feature, and patients with episodes in which amnesia 
was sometimes accompanied by other more familiar manifestations of epilepsy such as a brief loss of awareness and olfactory hallucinations. Exclusion of such patients, who were otherwise clinically indistinguishable, would have given a misleading picture of the spectrum of cases with this predominantly amnesic presentation of epilepsy. However, cases were excluded when witness accounts were unavailable, unreliable or indicated more extensive cognitive impairment during all attacks (see Appendix D, cases B2 and B3 for examples). These patients generally had attacks that consisted of a typical complex partial seizure, characterised by unresponsiveness, followed by a period of postictal amnesia. They did not, therefore, meet our diagnostic criteria for TEA but instead resemble those patients, described by Gallassi (Gallassi, 2006; Gallassi et al., 1992; Gallassi et al., 1988a), with the epileptic amnesic syndrome (EAS) (see Chapter 1). Further research is required to clarify the relationship between such patients and those with TEA. Questions to address include whether features typical of TEA - such as the onset in later life, the association of attacks with waking, amnesic episodes commonly lasting around 30 minutes, olfactory hallucinations, and prominent interictal memory complaints - are also characteristic of the EAS. It is possible that, at the pathophysiological level, the two phenomena differ in degree rather than in kind. Nevertheless, the results presented in this chapter demonstrate that, at a clinical level, the concept of TEA is a useful heuristic, particularly for identifying those patients in whom epileptic amnesic episodes occur independently of clear-cut complex partial seizures.

From the present study, it is not possible to conclude anything about the prevalence of TEA. It is generally regarded as a rare condition. Among 114 cases meeting stringent criteria for TGA, which has an estimate incidence of around 10 per 100,000 per year, Hodges and Warlow (1990b) identified eight (7\%) who subsequently developed epilepsy. However, the incidence of TEA cases is likely to be much higher than 0.6 per 100,000 per year. Firstly, TEA episodes are often much briefer than TGA and may, therefore, go unreported. Secondly, the diagnostic criteria for TGA used by Hodges and Warlow (1990b) will exclude a substantial proportion of patients with TEA in whom careful questioning reveals "additional neurological features" such as a brief loss of responsiveness, automatisms or olfactory hallucinations. Amongst the TIME cases, only $20 \%$ were initially diagnosed as having TGA. Furthermore, it seems likely that our study captured only a small fraction of incident TEA cases 
during the recruitment period. As will be shown in Chapter 4, the average IQ of the TIME patients was 118 , more than one standard deviation above the population mean. Since this is likely to reflect recruitment bias, the subpopulation from which the TIME sample was drawn represents just $10 \%$ of the overall UK population.

In developed countries, the incidence of unprovoked seizures increases in older age and is greater for persons over 75 years than at any other age (Hauser et al., 1993; Olafsson et al., 2005). This increase is usually attributed in a large part to cerebrovascular disease. Analysis of CVRF data did not support the hypothesis that TEA is related to overt vascular events or covert cerebrovascular disease. Ischaemic heart disease, stroke, TIA, peripheral vascular disease, diabetes, hypertension and smoking were not overrepresented in the patient group. Cigarette smoking was, in fact, less common amongst patients, a finding likely to reflect differences in the demographics of the two groups: i) the TEA patients were recruited from all over the United Kingdom whereas the control group were mostly from Scotland where the prevalence of smoking is higher (Goddard, 2006); ii) the socio-economic profile of the groups is likely to differ - TEA patients have a high average IQ (see Chapter 4), associated with lower smoking rates (Goddard, 2006). Cardiac arrhythmias, cardiac valve disease and arterial aneurysms were significantly overrepresented in the patient group. However, the prevalence of each was low and the confidence intervals very wide. The negative results reported in this chapter do not rule out cerebrovascular disease as an important aetiological factor in TEA. Vascular risk factors, even without a previous history of stroke, play a significant role in the aetiology of epilepsy in older age (Li et al., 1997; $\mathrm{Ng}$ et al., 1993). Larger studies are needed to investigate their importance in TEA.

Although cerebrovascular disease is the most commonly identified aetiology for epilepsy in the elderly (Ramsay et al., 2004; Sander et al., 1990), neurodegenerative pathologies such as Alzheimer's disease, head injury, tumours and systemic and metabolic disease also account for a substantial number of cases (Cloyd et al., 2006). None of these factors was common amongst the TEA patients in this study, although longer-term follow up will be needed to determine whether TEA is, in some cases, an early manifestation of neurodegenerative disease. Between $25 \%$ and $45 \%$ of cases of new onset epilepsy in patients over the age of 60 have no obvious aetiology (Cloyd et 
al., 2006; Hauser et al., 1993). At present, the majority of TEA cases fall into this group.

The nature of the amnesia in TEA, together with the EEG findings of this and other studies (Palmini et al., 1992; Vuilleumier et al., 1996), and the high frequency of olfactory hallucinations, point towards dysfunction in the medial temporal lobes. This region is known to play a key role in the acquisition of new memories and the retrieval of recent - and possibly remote - episodic events (Squire, 1992). It is reasonable to hypothesise, therefore, that the amnesic attacks of TEA are due to transient dysfunction in the hippocampus and related medial temporal cortices. However, significant memory impairment usually only occurs in the context of bilateral hippocampal impairment. In TLE, seizure discharges generally propagate first to ipsilateral temporal neocortex and only then to contralateral structures (Lieb et al., 1986; Spencer et al., 1987). This widespread dysfunction results in confusion and loss of responsiveness during complex partial seizures. It has been proposed (Gloor et al., 1993; Palmini et al., 1992) that in pure amnesic seizures, where consciousness is retained, ictal discharges may propagate to the contralateral hemisphere via the dorsal hippocampal commissure, without involving temporal neocortex. This interesting hypothesis is yet to be confirmed.

Cognitive dysfunction temporally associated with epileptic seizures can be caused either by ictal neuronal hyperactivity or by postictal depression of activity. There is electrographic evidence that both can give rise to TEA (Lee et al., 1992; Palmini et al., 1992; Tassinari et al., 1991; Vuilleumier et al., 1996). One patient in this series (case 37) had an amnesic episode whilst undergoing electroencephalography. A brief $(<1$ minute) burst of left temporal spikes, during which the patient was unresponsive to speech, was followed by normalization of the EEG and a 10-minute period of amnesia characterised by repetitive questioning about recent events. Although many TEA episodes are longer than is usually expected for temporal lobe seizures, persistent electrographic seizure activity has been reported during protracted epileptic amnesia (Lee et al., 1992; Tassinari et al., 1991; Vuilleumier et al., 1996), and it is recognised that both seizures and postictal dysfunction can be prolonged in older patients (Rowan, 2000). The question of whether episodes of TEA are ictal or postictal is discussed further in Chapter 3. 


\subsection{Summary}

In summary, this large, prospectively recruited group of patients meeting diagnostic criteria for TEA demonstrated many of the features that have previously been observed in smaller studies. Strikingly, just one quarter of patients were correctly diagnosed with epilepsy after their first consultation with a specialist physician. Whilst episodes of TEA can be indistinguishable from TGA, they are recurrent, usually briefer, occur upon waking and may involve an incomplete anterograde amnesia. In addition, they are frequently associated with olfactory hallucinations and complaints of interictal memory impairment. The results of this study did not support earlier suggestions that cerebrovascular risk factors might play a role in the aetiology of TEA. In the following chapter, I compare the TIME Project results with the features of previously reported cases. 


\section{CHAPTER THREE}

\section{A comprehensive review of transient epileptic amnesia}

\subsection{Introduction}

In Chapter 2, I described the clinical features of the 50 cases of transient epileptic amnesia (TEA) recruited to the TIME Project: the patients' demographics, the nature of the amnesic attacks, the persistent subjective memory impairment, the results of electroencephalography, and the response to treatment. In many respects, these findings confirmed careful observations by previous investigators of individual patients and small series of cases. In this chapter, I report the results of a comprehensive review of the literature in which I identified all reported cases meeting the diagnostic criteria for TEA. These findings are combined with those from the TIME Project in order to provide the most reliable description possible of the syndrome and form a basis for detailed discussion of the characteristic features.

\subsection{Methods}

An exhaustive search of the medical and psychological literature was performed to identify case reports and case series of transient amnesia associated with epilepsy. The search encompassed publications indexed in MEDLINE (from 1966), EMBASE (from 1980) and PSYCHINFO (from 1967) prior to the end of November 2007. The titles and available abstracts of pertinent papers were scrutinised and further hand searching of reference lists was used to identify un-indexed reports. For a case to be included, the diagnostic criteria used in the TIME Project (Butler et al., 2007; Zeman et al., 1998), and described in Chapter 2, had to be met.

The cases were then studied for information about the following: i) demographics, ii) aetiology, iii) clinical features of attacks, iv) anatomico-physiological underpinnings of the syndrome, v) interictal memory deficits and the relation to other clinical features, vi) prognosis. 


\subsection{Results and Discussion}

The literature search resulted in 93 case reports in 45 papers. Of these, 54 cases, in 28 papers, that met the diagnostic criteria were identified. The remaining 39 cases were discarded due to incomplete description (21 cases); single episode (eight cases); no witness (five cases); evidence of other cognitive impairment (three cases); no evidence of epilepsy (one case); and pure anomia rather than typical amnesia (one case). Of the 50 cases included in the TIME Study (Butler et al., 2007), 10 had been previously described (eight in Zeman et al. (1998), two in Manes et al. (2005). The final analysis therefore included: 50 cases from the TIME study ('TIME cases') and 44 from elsewhere in the literature ('literature cases').

TIME cases were prospectively recruited and assessed. The available data on clinical features, past medical and psychiatric history and neuropsychological test performance are therefore comparatively consistent. In contrast, the relative inconsistency of the data available from literature cases is reflected by variability in the denominator used when proportions are calculated below. Importantly, certain literature case reports offer valuable insight from clinical and electrophysiological data obtained during the amnesic attack. The principal clinical features of all identified cases are summarised in Table 3.1.

\subsubsection{Demographics of TEA}

The ages of onset of the amnesic attacks in the TIME and literature cases are shown in Figure 3.1. The mean age of onset was 57.2 years (literature cases $=55.1$ years; TIME cases $=62.1$ years). The range was 11 to 82 years in the literature cases and 44 to 77 years in the TIME cases. Nine literature cases had an onset before 40 years of age. From the available data, no other clinical features clearly distinguish this subpopulation. TEA is therefore usually a syndrome of middle to old age. This is similar to the age range of transient global amnesia (TGA), a syndrome which also results in transient impairment of declarative memory and is attributed to neuronal dysfunction in the medial temporal lobes, although the precise mechanism remains to be established. It is not clear why advancing age should predispose to transient dysfunction of the medial temporal lobes. Given the sensitivity of this region to 


\section{Chapter 3}

\begin{tabular}{|c|c|c|c|c|c|c|c|c|c|c|c|c|c|c|}
\hline Authors (year) & $\begin{array}{l}\text { Age } \\
\text { at } \\
\text { onset }\end{array}$ & Sex & $\begin{array}{l}\text { Duration } \\
\text { of } \\
\text { attacks }\end{array}$ & $\begin{array}{l}\text { Total } \\
\text { number }\end{array}$ & $\begin{array}{l}\text { Waking } \\
\text { attacks }\end{array}$ & $\begin{array}{l}\text { Ictal } \\
\text { AA }\end{array}$ & $\mathbf{R Q}$ & $\begin{array}{l}\text { Olf } \\
\text { aura }\end{array}$ & Automatisms & $\begin{array}{l}\text { "blank } \\
\text { spell" }\end{array}$ & $\begin{array}{l}\text { EEG } \\
\text { tve }\end{array}$ & Imaging & $\begin{array}{l}\text { Interictal } \\
\text { memory }\end{array}$ & $\begin{array}{l}\text { Response } \\
\text { to AED }\end{array}$ \\
\hline Lou (1968) & 61 & M & $30-60 \mathrm{~min}$ & 9 & no & yes & yes & no & no & no & $\mathrm{L}$ & & $\downarrow$ & complete \\
\hline Lou (1968) & 79 & $\mathrm{~F}$ & $1-24 \mathrm{hr}$ & 3 & & partial & yes & & & & B & & & \\
\hline Croft et al (1973) & 58 & $\mathrm{~F}$ & $1-24 \mathrm{hr}$ & 2 & no & yes & yes & & & & B & & & complete \\
\hline Morrell (1980) & 27 & M & & & & yes & & & & yes & $\mathrm{B}$ (ictal) & & & \\
\hline Shuping et al (1980) & 60 & M & $30-60$ min & 3 & & yes & & & & & $\mathrm{N}$ & L HC GBM & & died \\
\hline Dugan et al (1981) & 82 & M & $1-24 \mathrm{hr}$ & 3 & & yes & yes & & & no & B (ictal) & CT normal & & complete \\
\hline Deisenhammer (1981) & 11 & $\mathrm{~F}$ & $1-15 \min$ & 3 & yes & yes & yes & & & no & $\mathrm{R}$ & CT normal & & complete \\
\hline Meador et al (1985) & 47 & $F$ & $1-15 \min$ & 2 & no & partial & & & & no & B & R MTL meningioma & normal & complete \\
\hline Pritchard et al (1985) & 65 & M & $1-15 \min$ & 10 & & yes & & & & & B & CT normal & $\downarrow$ & complete \\
\hline Pritchard et al (1985) & 64 & M & $30-60 \mathrm{~min}$ & 3 & & yes & & & & & B & & $\downarrow$ & complete \\
\hline Kapur et al (1986/1989) & 74 & M & $15-30 \mathrm{~min}$ & 20 & yes & yes & no & & oral & yes & $\mathrm{B}$ & MRI normal & $\downarrow$ & \\
\hline Gallassi et al (1986) & 64 & M & $30-60 \mathrm{~min}$ & 25 & yes & yes & yes & no & oral & yes & NS & mod gen atrophy & $\downarrow$ & partial \\
\hline Miller et al (1987) & 22 & M & $1-15 \mathrm{~min}$ & & & & & & & yes & $\mathrm{L}$ & & & complete \\
\hline Miller et al (1987) & 62 & M & $15-30 \mathrm{~min}$ & 8 & yes & & yes & no & no & no & B & & & complete \\
\hline Gallassi et al (1988b) & 67 & $\mathrm{~F}$ & $1-15 \mathrm{~min}$ & 60 & yes & yes & yes & & oral & yes & NS & CT normal & $\downarrow$ & complete \\
\hline Gallassi et al (1988b) & 65 & M & 15-30min & 9 & & yes & & & oral & yes & NS & no & normal & complete \\
\hline Stracciari et al (1990) & 70 & $F$ & $1-15 \mathrm{~min}$ & 13 & & yes & yes & & oral & yes & $\mathrm{L}$ & MRI normal & normal & complete \\
\hline Tassinari et al (1991) & 57 & M & $1-15 \mathrm{~min}$ & & & yes & yes & yes & oral & yes & B (ictal) & & & complete \\
\hline Palmini et al (1992) & 30 & $\mathrm{~F}$ & $<1 \min$ & & & yes & no & no & manual & no & B (ictal) & L HS & & continued \\
\hline Palmini et al (1992) & 36 & $\mathrm{~F}$ & $<1$ min & & & yes & & no & & & $\mathrm{B}$ (ictal) & atrophy L HC/AG & & continued \\
\hline Palmini et al (1992) & 44 & $\mathrm{~F}$ & $<1 \mathrm{~min}$ & & & yes & & no & & no & $\mathrm{B}$ & normal & & continued \\
\hline Kapur (1993b) & 61 & $\mathrm{~F}$ & 15-30min & 2 & yes & no & & no & no & & NS & $L$ frontal infarct & & complete \\
\hline Kapur (1993b) & 63 & $\mathrm{~F}$ & $1-24 \mathrm{hr}$ & 35 & yes & yes & no & no & oral & no & NS & CT normal & $\downarrow$ & partial \\
\hline Kapur (1993b) & 67 & $F$ & $30-60 \mathrm{~min}$ & 6 & no & partial & no & no & no & & NS & CT normal & normal & complete \\
\hline Kapur (1993b) & 28 & M & $>24 \mathrm{hr}$ & 20 & yes & yes & yes & no & oral & no & $\mathrm{B}$ & & $\downarrow$ & complete \\
\hline Kopelman et al (1994) & 54 & M & $30-60 \mathrm{~min}$ & 9 & & yes & yes & & manual & no & B & MRI normal & $\downarrow$ & complete \\
\hline Meo et al (1995) & 69 & $\mathrm{~F}$ & $1-24 \mathrm{hr}$ & 3 & yes & yes & no & & & yes & L (ictal) & & & complete \\
\hline Cammalleri et al (1996) & 47 & M & $<1 \mathrm{~min}$ & 4 & no & no & no & no & no & no & $N$ & angioma R cing & normal & complete \\
\hline Vuilleumier et al (1996) & teens & $\mathrm{F}$ & $1-24 \mathrm{hr}$ & 40 & yes & yes & no & & & no & B (ictal) & MRI normal & normal & complete \\
\hline Dasheiff (1997) & $\mathrm{m} /$ age & $F$ & & & no & Y/N & no & no & no & no & B (ictal) & & & no \\
\hline Hawley et al (1997) & 38 & $\mathrm{~F}$ & & & & yes & & & & yes & $\mathrm{L}$ & & & complete \\
\hline Zeman et al (1998) & 68 & M & $1-24 \mathrm{hr}$ & 5 & yes & partial & yes & yes & & no & B & CT normal & $\downarrow$ & complete \\
\hline Zeman et al (1998) & 79 & M & $1-24 \mathrm{hr}$ & 3 & yes & & yes & & & & B & post CC infarct & & \\
\hline Corridan et al (2001) & 69 & M & & 11 & yes & yes & yes & no & no & no & B & & $\downarrow$ & complete \\
\hline Mendes (2002) & 45 & M & $30-60 \mathrm{~min}$ & & & yes & no & & & & & CT normal & & partial \\
\hline Mendes (2002) & 71 & $F$ & $30-60 \mathrm{~min}$ & 4 & no & partial & yes & no & no & no & $N$ & MRI normal & & complete \\
\hline Mendes (2002) & 74 & M & $30-60 \mathrm{~min}$ & 4 & no & partial & yes & no & no & no & NS & MRI normal & & complete \\
\hline Engmann and Reuter (2003) & 48 & M & $>24 \mathrm{hr}$ & 3 & no & yes & & no & no & yes & $\mathrm{L}$ & MRI mild atrophy & & complete \\
\hline Maheu et al (2004) & 27 & M & $>24 \mathrm{hr}$ & 10 & & yes & no & & & no & $\mathrm{R}$ (ictal) & R MTL bleed & $\downarrow$ & \\
\hline Manes et al (2005) & 48 & M & $30-60 \mathrm{~min}$ & 4 & & & & & & & B & CT normal & & \\
\hline Manes et al (2005) & 64 & M & $<1 \min$ & 7 & & & & & & & B & CT normal & & \\
\hline Manes et al (2005) & 69 & M & $30-60 \mathrm{~min}$ & 5 & & & & & & & & normal & & \\
\hline Manes et al (2005) & 57 & M & $30-60 \mathrm{~min}$ & 6 & & & & & & & & MRI CVD & & \\
\hline Manes et al (2005) & 46 & M & $30-60 \mathrm{~min}$ & 4 & & & & & & & $\mathrm{R}$ & MRI normal & & \\
\hline $\begin{array}{l}50 \text { cases of TEA from } \\
\text { TIME (Butler et al., 2007) }\end{array}$ & $\begin{array}{l}62.1 \\
(44- \\
77)\end{array}$ & $\begin{array}{l}34 \\
M\end{array}$ & $30-60 \mathrm{~min}$ & 10 & $37 / 50$ & $\begin{array}{l}28 / 50 \\
\text { partial }\end{array}$ & $25 / 50$ & $21 / 50$ & $18 / 50$ & $12 / 50$ & $18 / 49$ & 1 meningioma & $\begin{array}{l}\text { ALF: } \\
22 / 50 \\
\text { RMI: } \\
35 / 50 \\
\text { TA: } \\
18 / 50\end{array}$ & $\begin{array}{l}46 / 48 \\
\text { complete }\end{array}$ \\
\hline
\end{tabular}


hypoxic damage and its situation at a 'watershed' between anterior and posterior circulation, it seems possible that cell damage secondary to vascular insufficiency may act as an epileptic focus.

The sex ratio of TEA cases was approximately two males to one female in both the literature cases (27 males, 17 females) and the TIME cases (34 males, 16 females). The overall percentage of males was $64 \%$ (95\% confidence interval $(\mathrm{CI})=55 \%$ to 74\%). The reasons for this sex difference are not clear. A recent of review of 1333 published cases of TGA (Quinette et al., 2006) found no significant sex difference. The proportion of males with TGA was $46 \%$, significantly different from the proportion in TEA patients $\left(\chi^{2}=3.62, \mathrm{p}<0.001\right)$.

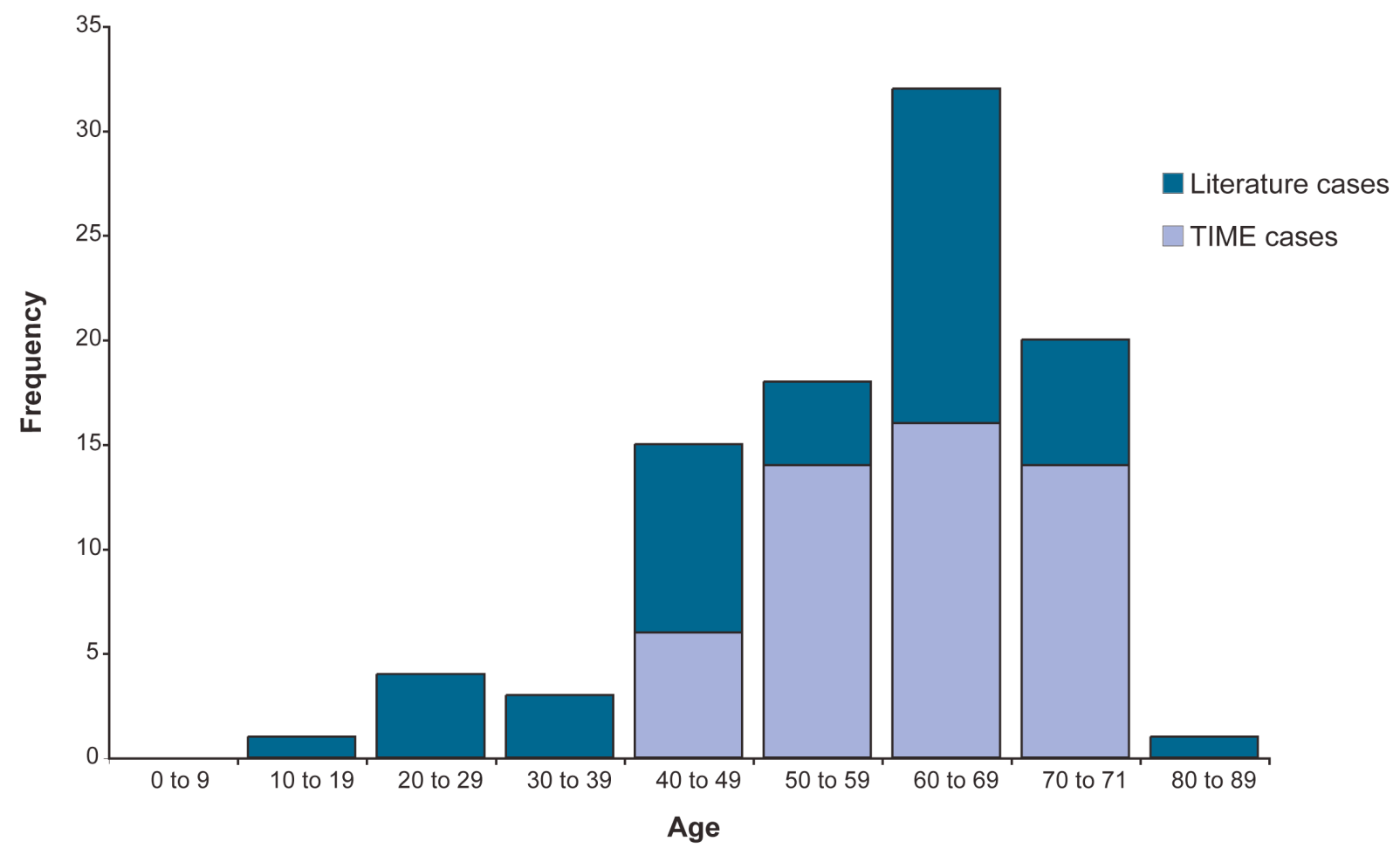

Figure 3.1: Frequency distribution of age of onset amongst TIME and literature cases of TEA 


\subsubsection{Duration}

The typical duration of amnesic attacks for each case was categorised as follows: $<1 \mathrm{~min}$; 1-15min; 15-30min; 30-60min; 1-24hr and $>24 \mathrm{hr}$. The distribution of durations across all identified cases of TEA is shown in Figure 3.2.

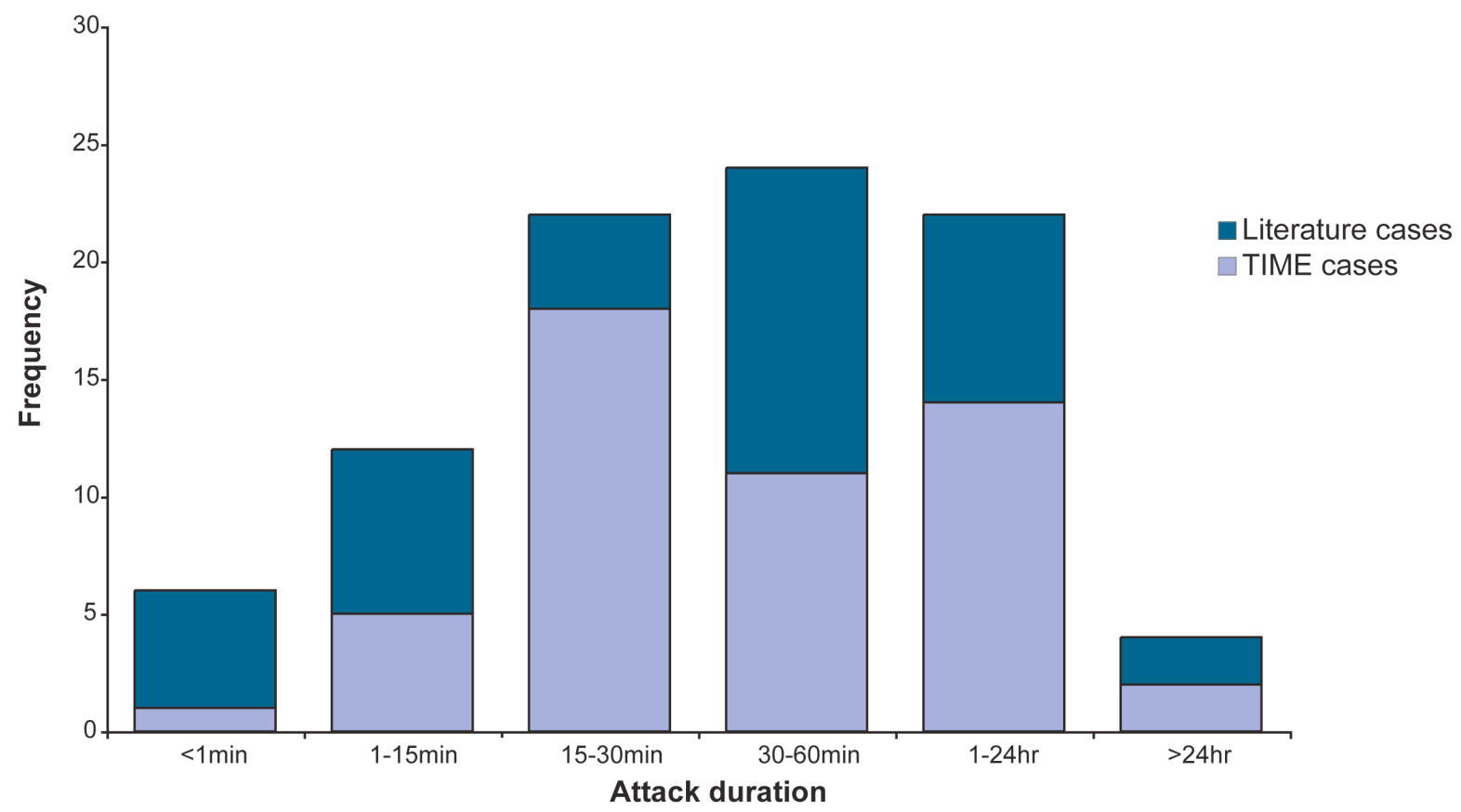

Figure 3.2: Frequency distribution of attack duration amongst TIME and literature cases of TEA.

The median duration was 30-60 min for literature cases, TIME cases and the group as a whole. This is briefer than the typical duration of TGA (4 to 10 hours) and confirms previous observations (Zeman et al., 1998). Of note, however, 23 cases (24\%) had attacks of similar duration to TGA, which could lead to diagnostic confusion. Very brief attacks $(<1 \mathrm{~min})$ are less likely to be noticed by the patient or observers. It remains to be explained why the majority of attacks are longer than would be expected for temporal lobe seizures and why some persist for several days. As mentioned in Chapter 2, it is recognised that both seizures and the post-ictal period can be prolonged in elderly patients (Rowan, 2000). 


\subsubsection{Frequency}

Before treatment, the overall mean frequency of attacks was 14.8/year (literature cases $=19.7 /$ year; TIME cases $=13.6 /$ year). This is higher than the figure of $3 /$ year previously reported in a smaller group of patients (Zeman et al., 1998) and distinguishes TEA from TGA in which the recurrence rate is low (around 3\% per year (Hodges and Warlow, 1990a)). However, the range across individuals is wide $(<1$ to $>60$ attacks per year) and a low frequency of attacks does not preclude a diagnosis of epilepsy. There is, moreover, a likelihood that attacks will be underreported, particularly those that are not witnessed by another person.

\subsubsection{Amnesia on awakening}

An association between amnesic attacks and arousal from sleep was noted in $70 \%$ of TEA cases (13/21 literature cases and 37/50 TIME cases). In 11/50 TIME cases, amnesic attacks occurred exclusively upon waking. The close relationship between sleep and epilepsy is well recognised. Primary generalised epilepsies are particularly associated with sleep, with purely nocturnal seizures occurring in $45 \%$ of cases (Janz, 1962), and seizures upon or shortly after waking being a characteristic feature of both juvenile myoclonic epilepsy (JME) and absence epilepsy. Among the focal epilepsies, nocturnal seizures are particularly common in frontal lobe epilepsy whereas in temporal lobe epilepsy the percentage is much lower. In an electroencephalographic study of patients with medically intractable focal epilepsy, $61 \%$ of frontal seizures but only $10 \%$ of temporal lobe seizures were recorded during sleep (Crespel et al., 1998). In general, both seizures and interictal epileptiform discharges are predominantly associated with the highly synchronised brain activity of non rapid eye movement sleep rather than the desynchronised state of rapid eye movement sleep (Sammaritano et al., 1991). The reason for the close relationship of TEA with sleep is unclear. It may be that the transition from sleep to waking acts as a trigger to a seizure focus in the medial temporal lobe. Alternatively, amnesia upon waking may reflect persistent post-ictal dysfunction of medial temporal lobe structures following a seizure during sleep. In one TIME case, for example, morning amnesia was always preceded by a brief arousal at around $2 \mathrm{am}$ when the patient sat up in bed, staring and said "Oh, the smell, the smell" before going straight back to sleep (case 83). 


\subsubsection{Ictal amnesia}

Anterograde amnesia is usually understood as a deficit in memory encoding or in storage mechanisms whereas retrograde amnesia, as least insofar as it proves transient, can be thought of as a memory retrieval problem. The relative impairment of anterograde and retrograde memory during TEA attacks, despite being difficult to ascertain from a retrospective patient or witness report, may therefore provide clues about the anatomy and degree of neuronal dysfunction. In TGA, anterograde amnesia is complete and the patient later has no recollection of events that occurred during the episode. In contrast, $44 \%$ of patients with TEA (10/36 literature cases; 28/50 TIME cases) describe at least partial preservation of anterograde memory during attacks and afterwards may report "being able to remember not having been able to remember". A small number of TEA patients (2/36 literature cases; 3/50 TIME cases) are able to remember their attacks in rich subjective detail, suggesting minimal impairment of encoding. This later recollection of ictal events does not necessarily correlate with the apparent anterograde amnesia during the attack. The three TIME cases referred to here repetitively questioned witnesses during their amnesic attacks. Vuilleumier et al. (1996) describe a patient who, during non-convulsive status epilepticus, could not perform anterograde memory tasks but who nevertheless encoded events into longterm memory. This presumably reflects temporary impairment of memory retrieval but preserved encoding and storage. In contrast, Dasheiff et al. (1997) describes a patient who, during electrographic seizures recorded with both scalp and invasive EEG monitoring, performed normally on memory tests but was subsequently amnesic for these episodes.

The degree of retrograde amnesia experienced during an episode of TEA also varies across individuals. Of 22 classifiable cases in the TIME study, 12 were associated with extensive ictal retrograde amnesia, stretching years into the past, whereas 10 appeared to have an ictal retrograde amnesia that was limited to events of the most recent days or weeks. Five of 33 cases in the literature are described as having no apparent retrograde memory deficit during the attack, although none of them had formal retrograde memory testing during the ictus. Such cases of apparently pure anterograde amnesia may go unnoticed as the patient will remain orientated and behave normally during the attack unless very recent memory is probed. The 
possibility that attacks of TEA irreversibly disrupt retrograde memory is not mentioned in any of the literature case reports but three TIME study patients volunteered that their TEA attacks permanently 'erased' memories of the preceding 24 hours.

\subsubsection{Repetitive questioning}

In TGA, the patient will repeatedly ask observers questions such as: "Where am I?" "What day is it?" or "What is happening to me?" In TEA, however, this is a feature in just over 50\% (17/30 literature cases; 25/50 TIME cases). Interestingly, the presence of repetitive questioning does not predict the degree of later recollection of ictal events $\left(\chi^{2}=0.325, \mathrm{p}=0.569\right)$.

\subsubsection{Additional features}

In $65 \%$ of cases (16/29 literature cases, 36/50 TIME cases), amnesic attacks were sometimes accompanied by additional features. However, 46/50 TIME cases had at least one attack with memory loss as the only feature.

Olfactory hallucinations: Amongst the TIME cases, olfactory or gustatory hallucinations were the most common additional feature and were reported by $42 \%$ (21/50) of patients upon direct questioning. Of these, 16 cases reported a purely olfactory sensation, two purely gustatory and three a mixed olfactory/gustatory sensation. The odour or taste was described as unpleasant in all but one case. In contrast, olfactory or gustatory hallucinations were described in only $2 / 16$ literature cases. Three additional TIME cases complained of a greatly decreased sense of smell since the onset of their amnesic attacks. Olfactory and gustatory hallucinations are generally held to be a rare feature of epilepsy, and almost always associated with a temporal lobe focus. Estimations of frequency vary. Penfield and Perot (1963) found seven cases $(0.6 \%)$ in 520 temporal lobe seizure patients. Acharya et al. (1998) found only 13 patients $(0.9 \%)$ with olfactory auras among 1423 evaluated at the Cleveland Clinic between 1991 and 1996. Higher prevalence rates have been reported by Chen et al. (2003) (5.5\%), Ebner and Kerder (2000) (6.3\%) and Manford et al. (1996) (7.1\%). All studies except that by Manford et al. (1996) investigated only patients 
with medically intractable focal epilepsy. Several studies have reported a particularly high incidence of tumours among patients with olfactory auras, including Jackson and Beevor (1890), Penfield and Jasper (1954) and Acharya et al. (1998). The latter found a neoplastic lesion in 10 of the 13 patients in their study. Others, however, have disputed this and maintain that hippocampal sclerosis is the most commonly associated pathological finding (Chen et al., 2003; Fried et al., 1995). There is greater consensus about the approximate anatomical origin of epilepsy-associated olfactory hallucinations, with the majority of patients having a seizure focus in the anterior medial temporal lobe. Hughlings-Jackson localised olfactory and gustatory functions to the uncus. Olfactory hallucinations have also been provoked by direct cortical stimulation of the amygdala, rather than the hippocampus, in several studies (Andy et al., 1975; Bartolomei et al., 2004; Fergusson et al., 1969; Gloor et al., 1982; Jasper and Rasmussen, 1958). The hallucinations were, however, only rarely elicited, and it remains uncertain whether spontaneous events have the same precise anatomical origin.

Déjà $v u$ : Déjà vu is a common experience amongst healthy individuals as well as being associated with a variety of neurological and psychiatric conditions, among them temporal lobe epilepsy (Warren-Gash and Zeman, 2003) in which prevalence estimates vary widely (from $6 \%$ to $80 \%$ (Brown, 2003)). Given the probable involvement of medial temporal lobe structures in TEA, it is perhaps surprising that just five (10\%) TIME cases described experiencing frequent déjà vu, usually on occasions distinct from their amnesic attacks. This symptom was not reported in any of the literature cases. Ictal déjà vu may involve inappropriate activation of brain circuits underlying familiarity. Cortical stimulation studies have elicited déjà vu from stimulation in the anterior medial temporal lobes, more frequently on the right than the left in right-handed patients (Bancaud et al., 1994; Gloor et al., 1982; Halgren et al., 1985). A recent study (Bartolomei et al., 2004) found déjà vu to be more frequently produced by stimulation of the entorhinal cortex than the hippocampus or amygdala. The frequency of 'physiological' déjà vu declines with age, but it is otherwise unclear why TEA, which is likely to arise from structures in which epileptic activity can give rise to déjà vu, should so rarely be associated with it. 
Automatisms: Automatisms (involuntary, semi-purposeful movements) were reported to accompany some amnesic attacks in $40 \%$ of patients (10/19 literature cases, $18 / 50$ TIME cases). Oro-alimentary automatisms (chewing or lip smacking) were the most common (8/10 literature cases, 14/18 TIME cases) and the remainder were manual automatisms. Oral automatisms are significantly associated with a temporal rather than frontal seizure focus (Manford et al., 1996) and with hippocampal rather than extra-hippocampal temporal lobe seizures (Gil-Nagel and Risinger, 1997).

Unresponsiveness: A brief period of unresponsiveness was sometimes associated with the amnesic episode in $28 \%$ of patients (10/28 literature cases, 12/50 TIME cases). Unresponsiveness, one of the hallmarks of 'altered consciousness' in complex partial seizures, is thought to represent bilateral spread of seizure activity (Bancaud et al., 1994; Blumenfeld and Taylor, 2003; Inoue and Mihara, 1998; Lux et al., 2002). When this occurs at the beginning of the episode, it may indicate that the subsequent memory impairment is a post-ictal phenomenon (Morrell, 1980).

Other seizure types: Patients with TEA may also experience seizures in which amnesia is not a prominent feature. These occurred in $21 \%$ of cases (13/39 literature cases, 6/50 TIME cases) and were almost always complex partial seizures. This observation raises the possibility that episodes of transient amnesia might be more widespread among TLE patients than is generally recognised, but that they go unnoticed or unreported. Generalised tonic-clonic seizures were only reported in 3/94 cases $(3 \%)$.

\subsubsection{Electroencephalography}

Interictal EEG: Interictal epileptiform abnormalities on electroencephalography (EEG) were seen in 43\% cases (23/44 literature cases, 18/49 TIME cases). Of these (TIME and literature cases combined), 31\% were left-sided, 12\% were right-sided and $56 \%$ were bilateral. All were localised over the temporal or frontotemporal region. The interictal EEG was entirely normal in $19 \%$ of cases (3/44 literature cases, 15/49 TIME cases). Surface EEG lacks sensitivity to discharges originating in the medial temporal lobes and the detection rate in TEA, given variable usage of routine or sleep EEG recording, is similar to that in other forms of temporal lobe epilepsy. 
Ictal EEG: Surface EEG recording during an amnesic attack was performed in nine literature cases and one TIME case. All recordings showed seizure activity, which in $8 / 10$ cases involved both temporal lobes and in the others remained unilateral (one left- and one right-sided). Amnesia was observed as an ictal phenomenon in six cases and as post-ictal in four cases.

\subsubsection{Brain imaging}

Brain imaging results were available for 29/44 literature cases $(C T=14, \mathrm{MRI}=15)$ and 49 TIME cases $(\mathrm{CT}=2, \mathrm{MRI}=47)$, as will be discussed in detail in Chapter 6 . Focal lesions were detected in 7/78 cases of which four were likely to have played an aetiological role in the seizures $(5 \%)$. These were: two right temporal lobe meningiomas (Meador et al., 1985 and TIME case 40), haemosiderin deposition in the right medial temporal lobe (Maheu et al., 2004) and a glioblastoma multiforme in the left hippocampal region (Shuping et al., 1980). We have recently observed medial temporal lobe high signal on T2 weighted MRI sequences in two patients with particularly frequent episodes of TEA, with unilateral hippocampal hypermetabolism on a PET scan in one of these, resolving when the seizures subsided (see Chapter 7 and Butler and Zeman, 2008a). In general, therefore, TEA is not associated with clinically detectable, focal brain lesions, but where these are present they involve medial temporal regions.

\subsubsection{Pathophysiology}

The amnesic attacks seen in TEA may be due to ictal or post-ictal neuronal dysfunction. The possibility that they are due to persistent epileptic activity (nonconvulsive status epilepticus) is supported by accounts of ongoing or intermittent automatisms (Kapur, 1993b) and the cases of Lee et al. (1992), Meo et al. (1995) and Vuilleumier et al. (1996). Lee et al. (1992) describe a 38-year old woman who suffered a 12-day episode of pure amnesia accompanied by persistent epileptic activity isolated to the left temporal region. Meo et al. (1995) report a 69-year old female with an amnesic episode lasting several hours during which ictal discharges originating from the right temporo-central region were recorded. In both these cases, 
the patients' behaviour during bursts of ictal discharges was indistinguishable from that observed during interictal activity. Vuilleumier et al. (1996) describe a 41-year old woman with persistent failure of recollection for 10 hours during which EEG revealed continuous generalised epileptic activity with phase reversal in bilateral frontotemporal regions. However some prolonged TEA attacks may result from prolonged post-ictal amnesia or a "Todd's paralysis of memory" (Morrell, 1980) presumably following a brief or subclinical period of seizure activity - as in the cases described by Morrell (1980) and Tassinari et al. (1991). Morrell (1980) reports a $27-$ year old patient in whom bilateral hippocampal seizures, recorded by depth electrodes, were followed by a postictal period of amnesia of which the duration was related to the duration of the preceding ictus. Tassinari et al (1991) describe the case of a 60-year old man who, during a 90-minute period of video-EEG monitoring, experienced two seizures, each lasting about one minute, that were characterised by olfactory hallucinations, oro-alimentary automatisms and a brief loss of responsiveness and were associated with bilateral temporal paroxysmal activity on the EEG. Between the seizures, and for a further three hours, the patient exhibited a mixed anterograde and retrograde amnesia with repetitive questioning. Interestingly, one minute prior to the onset of the second seizure, there was an abrupt recovery of memory function. Further study is needed to determine the relative frequencies of ictal and postictal amnesia in TEA and whether the difference in physiological state is reflected in the neuropsychological profile of the patient.

\subsubsection{Aetiology}

Besides rare structural abnormalities, the cause of TEA is usually obscure. (Zeman et al., 1998) pointed out that a history of cardiac disease was common in their series, and hypothesised that cardiac-related hypoxic damage to medial temporal lobe structures might have caused the epilepsy. Amongst the TIME cases, however, there was no excess of cardiovascular or cerebrovascular disease risk factors (see Chapter 2).

\subsubsection{Interictal memory disturbance}

Complaints of interictal memory dysfunction are common in TEA and were noted in $80 \%$ patients (16/17 literature cases, 38/50 TIME cases). The most common problems 
reported were i) a patchy loss of memories for remote, personally experienced events (35/50 TIME cases), ii) accelerated forgetting over days to weeks of newly acquired memories (22/50 TIME cases) and iii) topographical memory deficits (18/50 TIME cases).

The interictal neuropsychological data from the TIME cases are discussed in the following chapter. Neuropsychological test data are reported in 23/44 literature cases. In 11 of these, no evidence of memory impairment was found, although testing was limited to the Addenbrooke's Cognitive Examination, a brief screening instrument, in five cases (Manes et al., 2005). In a further eight cases, a range of standard tests revealed anterograde memory impairment for verbal and non-verbal material. In the remaining four cases, anterograde memory tests were largely normal but there was significant impairment in remote memory. Notably, however, three of these patients had a focal anterograde deficit in face recognition memory.

In a series of papers, Gallassi (Gallassi, 2006; Gallassi et al., 1990a; Gallassi et al., 1992; Gallassi et al., 1988a; Gallassi et al., 1988b; Gallassi et al., 1986) has described the 'epileptic amnesic syndrome', in which patients present with complaints of severe, persistent memory impairment in the context of usually subtle temporal lobe seizures (see Chapter 1). In some cases, these seizures are followed by a period of transient amnesia, which Gallassi, following Pritchard et al. (1985), has called 'epileptic amnesic attacks' (EAA). Clearly, the patients described by Gallassi have much in common with TEA patients and some of those for whom sufficient clinical data have been published have been included in the present review. The epileptic amnesic syndrome and TEA are not, however, coterminous as some patients with TEA do not have pronounced interictal memory disturbance and some patients with epilepsy and memory problems do not have transient amnesic attacks.

\subsubsection{Treatment responsiveness}

Complete cessation of transient amnesic episodes was achieved with anticonvulsant therapy in $88 \%$ patients (25/32 literature cases, 46/48 TIME cases). The remainder showed a substantial decrease in attack frequency. The reason for this excellent 
response rate is unknown but is generally reported in late-onset epilepsy (Stephen and Brodie, 2000).

\subsection{Summary}

The comprehensive review presented in this chapter reveals that the clinical features of TEA are broadly consistent across numerous independent reports published over the past 40 years. The literature cases as well as the TIME cases generally experienced the onset of amnesic attacks in late middle age, and had attacks that were brief, relatively frequent and often occurred upon waking. The semiology of the episodes and their association with olfactory hallucinations and oro-alimentary automatisms suggest a seizure focus in the medial temporal lobes. This hypothesis is supported by electroencephalography which, when positive, usually reveals epileptiform abnormalities over the temporal regions. Structural lesions are rarely revealed by neuroimaging. A number of clinically important questions relating to the aetiology, epidemiology and prognosis of TEA remain unanswered and should be addressed in future work. Complaints of persistent memory difficulties are common amongst patients, although standard memory testing in the literature cases was usually normal or revealed only mild impairment. In the following chapter, I report the results of neuropsychological testing in the 50 cases of TEA in the TIME Project. 


\section{CHAPTER FOUR}

\section{The neuropsychological characteristics of transient epileptic amnesia}

\subsection{Introduction}

In the previous two chapters, I have focussed on the principal clinical features of transient epileptic amnesia (TEA) and, in particular, on the characteristics of the amnesic attacks. However, many patients with TEA complain of significant ongoing problems with memory, even when the attacks themselves have ceased with anticonvulsant medication. Patients principally complain of three key problems: i) the excessively rapid decay, over days to weeks, of newly acquired memories accelerated long-term forgetting (ALF), ii) a patchy loss of memories for remote, salient autobiographical events such as holidays and weddings, and iii) new difficulty navigating around previously familiar environments. These problems have been noted in previous descriptions of TEA. In the TIME study, they were reported by $44 \%, 70 \%$ and $36 \%$ of patients respectively.

As discussed in Chapter 1, the nature and causes of interictal memory impairment in epilepsy are poorly understood, and complaints are often out of proportion with the degree of impairment seen on neuropsychological testing. It has been suggested that this subjective-objective mismatch is particularly pronounced in TEA, and that the problem lies in the inadequacy of standard neuropsychological instruments, which examine learning and retention over delays of up to one hour (Manes et al., 2005; Manes et al., 2001; Zeman et al., 1998). Certainly, previous evaluations of neuropsychological function in TEA have failed to demonstrate significant deficits on standard tests of anterograde memory (Kapur, 1990; Manes et al., 2005; Zeman et al., 1998), although mild impairment has been reported (Gallassi et al., 1988a; Gallassi et al., 1986; Kopelman et al., 1994; Pritchard et al., 1985). Novel tests may, therefore, need to be developed. One small study (Manes et al., 2005) examined long-term anterograde memory in seven patients with TEA and found recall of a short story to be normal after 30 minutes but impaired relative to healthy control subjects after a 
delay of six weeks. A larger study (Blake et al., 2000) reported similar findings in a group of patients with temporal lobe epilepsy (TLE). Manes et al (2001), in their detailed investigation of remote memory in a single patient with TEA, revealed a temporally extensive deficit in autobiographical memory in the context of apparently normal anterograde memory. Remote memory loss was also documented in the series of seven TEA patients studied by Manes et al (2005).

There is some evidence, therefore, that ALF and remote memory impairment are demonstrable features of TEA. However, large studies are lacking and the relationship of these phenomena to performance on standard neuropsychological tests and subjective assessments of memory is not clear. In this chapter, I report the results of detailed neuropsychological testing of the 50 TEA patients in the TIME Project. Topographical memory has not previously been examined in TEA and was not part of the present investigation.

\subsection{Methods}

\subsubsection{Subjects}

All 50 TEA patients recruited into the TIME Project underwent standard neuropsychological testing and assessment of autobiographical memory. Long-term forgetting was investigated in a subset of 24 patients ('subgroup 1'), selected for their normal performance on standard anterograde memory tests, lack of other evident cognitive deficits or psychiatric diagnoses and geographical proximity to the study centre. Of these 24 patients, 12 had subjective complaints of ALF and 12 did not. They did not differ significantly from the remaining patients on any other clinical variables.

Twenty-four age and education matched, neurologically normal control subjects were recruited. Seventeen of these were volunteers, usually a spouse or close friend, nominated by patients from subgroup 1 . Seven patients were unable to nominate their own controls. For these patients a similarly matched control subject was obtained from the volunteer panel of the Medical Research Council Cognition and Brain Sciences Unit at the University of Cambridge. Control subjects underwent a medical 
history interview, magnetic resonance imaging (MRI), and received the standard neuropsychological, ALF and autobiographical memory test batteries.

\subsubsection{Subjective memory questionnaires}

Self-completion questionnaires were administered, prior to neuropsychological testing, to all patients and controls in order to assess their own perception of their everyday memory and memory for remote, personally experienced events. In the everyday memory questionnaire (EMQ) (Thompson and Corcoran, 1992) (see Appendix E), respondents are asked to estimate the frequency of 18 everyday memory failures, such as misplacing objects around the house, forgetting people's names or having to repeatedly check whether a certain task has been completed. Ratings range from 0 ('not at all') to 5 ('more than once a day'), giving a maximum possible score of 90. In the very long-term memory questionnaire (VTLMQ) (see Appendix F), subjects are asked to rate how often they have forgotten 13 types of personally salient fact or event such as weddings, holidays, major projects or funerals. Frequency is scored from 0 ('never') to 4 ('many times'), giving a maximum possible score of 52.

\subsubsection{Standard neuropsychology}

Standard neuropsychological tests were used to assess a wide range of cognitive abilities including: general intelligence (Wechsler Abbreviated Scale of Intelligence (Wechsler, 1999)), visual perception (copy of the Rey-Osterreith complex figure (Osterreith and Rey, 1944)), anterograde memory (immediate and 30 minute delayed recall of a prose passage (from Wechsler Memory Scale-III (Wechsler, 1997)), 30 minute delayed recall of the Rey-Osterreith complex figure (Osterreith and Rey, 1944), the Recognition Memory Test (Warrington, 1984)), memory for famous faces (Graded Faces Test (Thompson et al., 2002)), language (Graded Naming Test (McKenna and Warrington, 1980)) and executive function (letter and category fluency, Wisconsin Card Sorting Test - 64 Card Version (Kongs et al., 2000)). This battery was administered on the occasion of the patient's clinical interview. Performance on each of the anterograde memory tests (delayed Logical Memory recall, Rey figure recall, word recognition memory and face recognition memory) was converted into a $\mathrm{z}$ score, i.e. the number of standard deviations the individual's score 
lay from the control group mean score. A summary measure of anterograde memory (zmem) was then obtained for each patient by calculating the average of these $\mathrm{z}$ scores.

\subsubsection{Accelerated long-term forgetting}

Memory retention over an extended delay of three weeks was examined using three types of material: a list of unrelated words, a series of meaningless, black and white designs and a short story (see Appendix G for the test material). The list of 15 words (from the Rey Auditory Verbal Learning Task (Schmidt, 1996)) was orally presented over a minimum of five and a maximum of 15 trials until the subject attained $90 \%$ accuracy at free recall. Learning to criterion helps eliminate potential differences in initial learning between patients and controls. Recall of the words was then tested at delays of 30 minutes, one week and three weeks. A similar procedure was used for reproduction of seven visually presented designs (from the Graham-Kendall Memory for Designs test (Graham and Kendall, 1968)) and for a short prose passage from the Rivermead Behavioural Memory Test (Wilson et al., 1991). Subjects were not forewarned about the one and three week probes, but were explicitly requested not to rehearse the material. Recognition memory was tested at the three-week delay for each stimulus set. For the word list, this involved a yes-no paradigm with 15 new words mixed with the 15 target words. Recognition memory for the designs and short story was tested with a forced choice paradigm (see Appendix G). Two equivalent versions of each test were available so that cohabitants did not receive the same material. Administration of each version was counterbalanced across the groups. Patients were asked to record any seizures or auras experienced between testing sessions. Long-term forgetting was defined as the percentage change in recall score between 30 minutes and three weeks. A summary measure of long-term forgetting (zALF) was calculated for each patient by averaging the long-term forgetting $\mathrm{z}$ scores for the word list, prose passage and designs.

\subsubsection{Autobiographical memory}

A semi-structured interview (the Modified Autobiographical Memory Interview (MAMI) see Appendix H) was used to assess memory for autobiographical facts and 
events relating to two topics for each decade of the subject's life. This test was developed from the widely used Autobiographical Memory Interview (AMI) (Kopelman et al., 1989). The modifications, in particular the probing of autobiographical memories from every life decade, were designed to address the criticism that the AMI is insensitive to mild or patchy impairment of autobiographical memory (Fujii et al., 2000; Manes et al., 2001). For each topic (e.g. 'holiday'), subjects were asked to answer five questions designed to probe semantic, or factual, memory (e.g. "How long did you stay?") and produce one detailed episodic memory (e.g. "Can you recall any incident, even if small, that occurred during your holiday in Seton Sands?"). Episodic memories were scored out of five according to their degree of specificity and experiential detail, based on the scheme described by Graham and Hodges (Graham and Hodges, 1997). A witness, usually a spouse, was present in all cases to corroborate the subject's account. The MAMI was administered to 48 patients in total. In this chapter, results are reported for 18 control subjects and 22 patients in subgroup 1, the intention being to investigate autobiographical memory impairment in TEA patients with normal performance on standard anterograde memory tests. Two patients in subgroup 1 and six control subjects were excluded from the analysis as they were unable to provide a witness to corroborate their accounts. Average scores across all decades were calculated for the semantic (MAMIs) and the episodic (MAMI-e) components.

\subsubsection{Statistical analysis}

The performance of TEA patients on standard neuropsychological testing was compared with that of controls using independent sample t-tests or the Mann-Whitney $\mathrm{U}$ test where appropriate. To compare long-term forgetting rates of the patient and control groups, repeated measures analyses of variance (ANOVA), with factors of participant group and delay (30 minutes, one week and three weeks), were carried out using recall score as the dependent variable. The Greenhouse-Geisser correction for non-sphericity was applied where necessary. Three-week recognition memory scores of the designs and story were compared with t-tests. Recognition of the word list was compared by performing a t-test on the d-prime statistic for each group. The d-prime statistic is a measure of accuracy derived from the ratio of true positives to false negatives. Semantic and episodic memory scores on the MAMI were obtained for 
each subject across the five most recent life decades and were analysed using repeated measures ANOVA, with factors of participant group and decade (most recent (Z), Z1, Z-2, Z-3, Z-4). Planned independent sample t-tests were used to explore the temporal extent of identified differences. Pearson correlation coefficients were calculated to investigate the relations between subjective memory and possible predictor variables. All analyses were performed using SPSS for Windows version 15.0 .

\subsection{Results}

\subsubsection{Standard neuropsychology}

Neuropsychological test results for patients and controls are shown in Table 4.1. The groups were well matched for age, education level and intelligence quotient (IQ). There were significant but small $(<1 \mathrm{SD})$ differences between the entire TEA patient group and controls on measures of anterograde memory (Logical Memory delayed recall $(\mathrm{p}=0.02)$ and recognition $(\mathrm{p}=0.048)$; delayed recall of the Rey-Osterreith complex figure $(p=0.037)$; recognition memory for words $(p=0.014)$ and faces $(p<$ 0.001)) and the depression rating of the Hospital Anxiety and Depression Scale (HADS) $(\mathrm{p}=0.006)$. HADS depression ratings did not correlate with scores on standard memory tests (delayed recall of Logical Memory story $(p=0.968)$ or Rey figure $(\mathrm{p}=0.962))$, and there was no significant difference in depression ratings between patients with and without self-reported interictal accelerated forgetting ( $\mathrm{p}=$ $0.77)$ or remote autobiographical memory loss $(p=0.60)$. The difference between patients and controls in performance on tests of semantic memory approached statistical significance (Graded Faces test: $p=0.053$; Graded Naming test: $p=0.088$; category fluency: $p=0.051$ ). No significant differences were detected on any aspect of the screening battery between the subset of patients selected for the study of accelerated long-term forgetting (subgroup 1) and their matched control subjects. 
Table 4.1: Demographic and neuropsychological profile of TEA patients and controls

\begin{tabular}{|c|c|c|c|}
\hline & $\begin{array}{c}\text { Patient subgroup } 1 \\
\begin{array}{c}(\mathbf{n}=24) \\
\text { mean }(S D)\end{array}\end{array}$ & $\begin{array}{l}\text { All patients } \\
(n=50) \\
\text { mean }(S D)\end{array}$ & $\begin{array}{c}\text { Controls } \\
(n=24) \\
\text { mean (SD) }\end{array}$ \\
\hline Age (years) & $67.0(8.7)$ & $68.3(8.6)$ & $67.7(8.1)$ \\
\hline Education (years) & $13.0(3.0)$ & $12.2(2.9)$ & $12.5(3.1)$ \\
\hline Full scale IQ & $124.3(10.4)$ & $118.3(12.8)$ & $120.0(14.4)$ \\
\hline \multicolumn{4}{|l|}{ Episodic memory } \\
\hline Story recall immediate (25) & $16.1(3.3)$ & $14.0(4.3)$ & $15.9(3.8)$ \\
\hline Story recall delayed (25) & $14.5(3.0)$ & $11.7(5.0)^{*}$ & $14.7(3.8)$ \\
\hline Story recognition (15) & $13.0(1.2)$ & $12.9(1.4)^{*}$ & $13.6(1.2)$ \\
\hline Rey figure delayed recall (36) & $16.3(5.8)$ & $15.0(6.5)^{*}$ & $18.6(6.1)$ \\
\hline Word recognition (50) & $47.8(2.0)$ & $46.1(4.7)^{*}$ & $48.3(1.9)$ \\
\hline Face recognition (50) & $43.3(3.9)$ & $40.7(5.4)^{* * *}$ & $45.1(2.9)$ \\
\hline \multicolumn{4}{|l|}{ Semantic memory } \\
\hline Graded faces $(60)$ & $41.3(8.9)$ & $40.0(9.6)$ & $44.0(7.6)$ \\
\hline Graded naming (30) & $23.2(3.0)$ & $21.4(5.1)$ & $23.5(4.2)$ \\
\hline \multicolumn{4}{|l|}{ Visuospatial perception } \\
\hline Rey figure copy (36) & $35.4(1.3)$ & $34.5(3.1)$ & $35.5(1.1)$ \\
\hline \multicolumn{4}{|l|}{ Executive function } \\
\hline Letter fluency (words/3min) & $47.9(11.4)$ & $42.5(13.9)$ & $43.8(11.4)$ \\
\hline Category fluency (words/min) & $21.9(5.4)$ & $19.3(5.9)$ & $22.0(4.4)$ \\
\hline WCST categories completed & $3.3(1.3)$ & $2.8(1.3)$ & $3.4(1.4)$ \\
\hline WCST total errors (\%ile) & $64.1(35.1)$ & $52.8(34.2)$ & $63.4(32.1)$ \\
\hline \multicolumn{4}{|l|}{ HAD } \\
\hline Anxiety score (21) & $6.0(4.9)$ & $5.4(3.5)$ & $4.7(2.8)$ \\
\hline Depression score (21) & $4.3(3.3)$ & $4.6(3.7)^{*}$ & $2.9(1.7)$ \\
\hline \multicolumn{4}{|c|}{$\begin{array}{l}\text { * significantly different from control group mean }(p<0.05) \\
\text { *** significantly different from control group mean }(p<0.001) \\
\text { HAD = Hospital Anxiety and Depression Scale; } I Q=\text { intelligent quotient; WCST = Wisconsin } \\
\text { Card Sorting Test }\end{array}$} \\
\hline
\end{tabular}




\subsubsection{Accelerated long-term forgetting}

Patients' and controls' performance on learning and delayed recall of the word list, designs and story is shown in Figure 4.1.

(a) Word list

\begin{tabular}{|c|c|c|}
\hline & $\begin{array}{l}\text { Patients }(n=24) \\
\text { mean (SD) }\end{array}$ & $\begin{array}{l}\text { Controls }(n=24) \\
\text { mean (SD) }\end{array}$ \\
\hline learning trials $(\mathrm{n})$ & $6.0(1.4)$ & $5.4(1.1)$ \\
\hline final learning trial recall (\%) & $97.4(3.5)$ & $98.5(2.9)$ \\
\hline 30 minute recall (\%) & $84.0(10.0)^{\star *}$ & $92.5(8.7)$ \\
\hline 1 week recall (\%) & $29.0(28.3)^{\star \star *}$ & $66.1(23.6)$ \\
\hline 3 week recall (\%) & $26.0(27.1)^{\star \star \star}$ & $63.3(26.4)$ \\
\hline 3 week recognition (d') & $1.77^{\star}$ & 2.63 \\
\hline
\end{tabular}

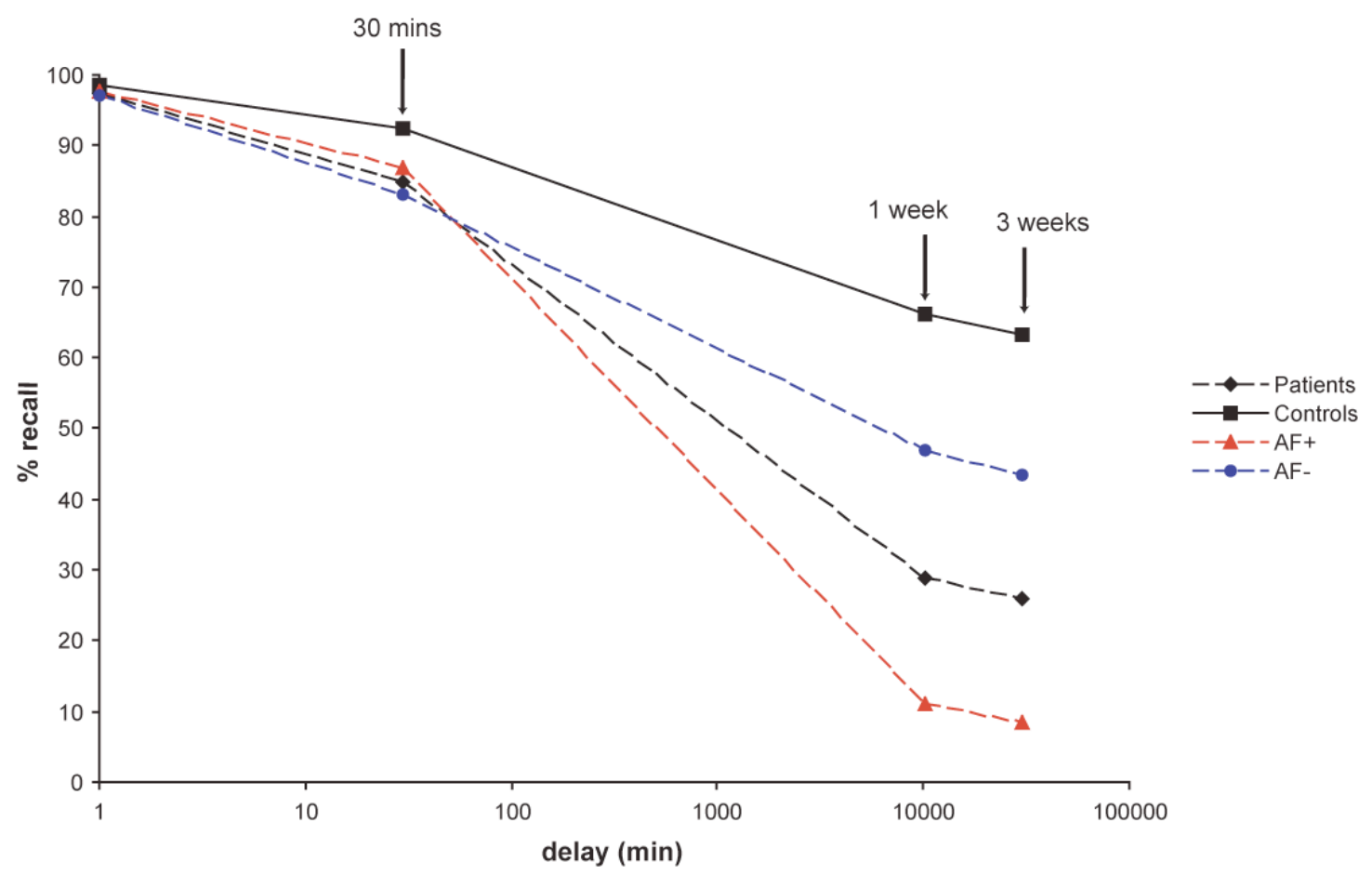


(b) Designs

\begin{tabular}{|c|c|c|}
\hline & $\begin{array}{l}\text { Patients }(n=20) \dagger \\
\text { mean }(S D)\end{array}$ & $\begin{array}{l}\text { Controls }(n=23) \dagger \\
\text { mean }(S D)\end{array}$ \\
\hline learning trials $(n)$ & $7.9(2.7)$ & $7.7(2.7)$ \\
\hline final learning trial recall (\%) & $96.0(4.5)$ & $94.6(4.0)$ \\
\hline 30 minute recall $(\%)$ & $91.9(9.9)$ & $91.5(6.7)$ \\
\hline 1 week recall $(\%)$ & $60.5(29.5)$ & $74.5(18.1)$ \\
\hline 3 week recall (\%) & $49.0(29.2)^{*}$ & $65.1(20.1)$ \\
\hline 3 week recognition (\%) & $73.9(22.2)$ & $76.1(22.4)$ \\
\hline
\end{tabular}

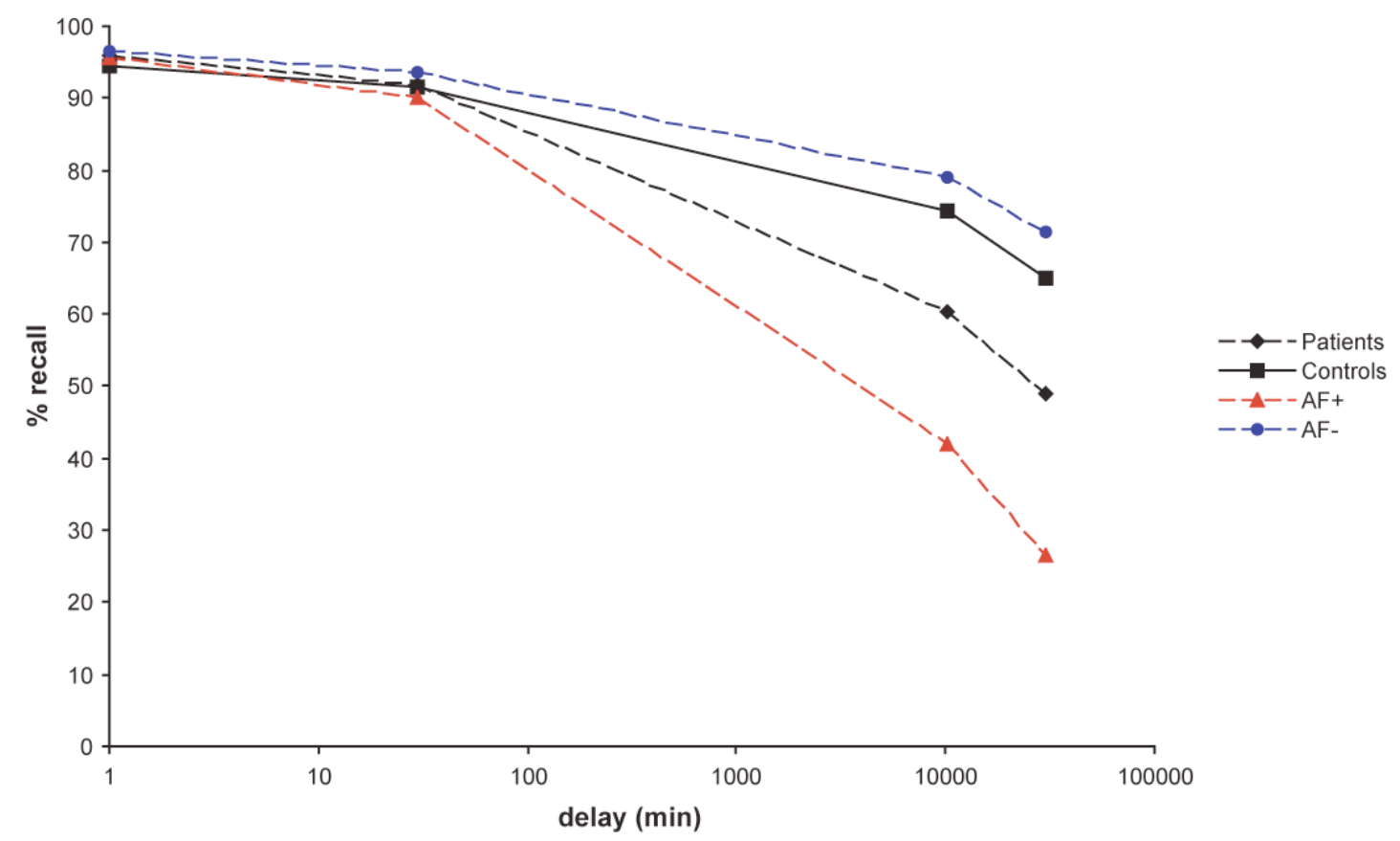


(c) Story

\begin{tabular}{lll}
\hline & $\begin{array}{l}\text { Patients }(\mathbf{n}=24) \\
\text { mean (SD) }\end{array}$ & $\begin{array}{l}\text { Controls }(\mathbf{n}=24) \\
\text { mean (SD) }\end{array}$ \\
\hline learning trials (n) & $8.0(2.5)$ & $7.2(2.8)$ \\
final learning trial recall (\%) & $91.4(2.6)^{\star *}$ & $93.9(3.3)$ \\
30 minute recall (\%) & $73.1(10.1)^{\star *}$ & $82.7(11.8)$ \\
1 week recall (\%) & $46.2(17.9)^{\star * *}$ & $67.6(13.7)$ \\
3 week recall (\%) & $42.0(17.6)^{\star * *}$ & $61.7(17.1)$ \\
3 week recognition (\%) & $85.8(16.6)^{*}$ & $94.2(5.7)$ \\
\hline
\end{tabular}

${ }^{*} p<0.05$

${ }^{* *} p<0.01$

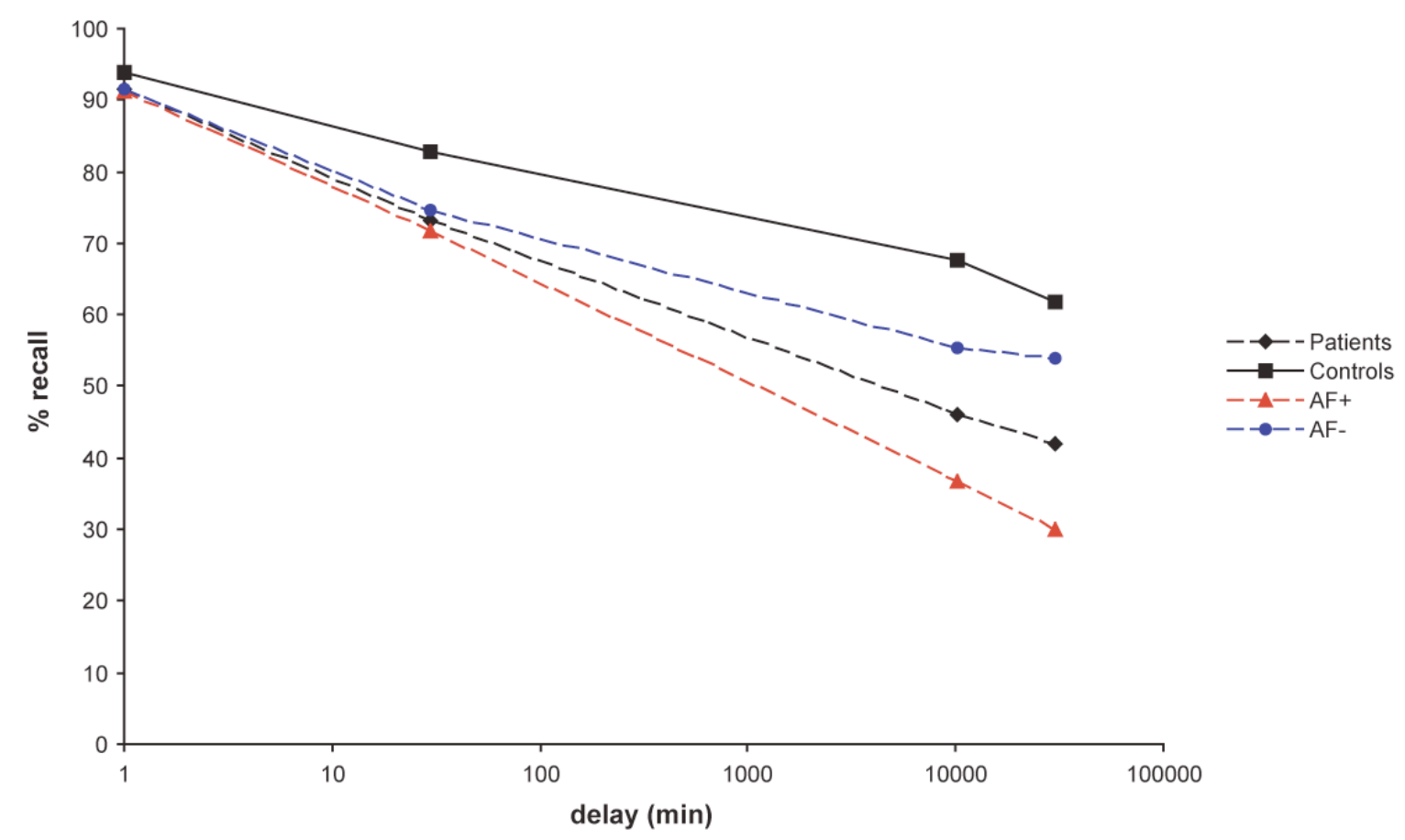

Figure 4.1: Performance of TEA patients and normal controls on learning and long-term recall of (a) the word list, (b) designs and (c) story. (AF+ = patients with subjective complaints of accelerated forgetting; AF- = patients without subjective complaints of accelerated forgetting). 
Mann-Whitney U tests revealed no significant differences between patient and control groups in the number of learning trials needed to reach the $90 \%$ criterion. At 30 minutes, patients recalled a mean of 12.6 words $(84.0 \%)$ and control subjects a mean of 13.9 words $(92.5 \%)$, a small but statistically significant difference $(\mathrm{p}=0.007)$. Between 30 minutes and one week there was a sharp drop in word recall in the patient group by comparison with controls $(\mathrm{p}<0.001)$, with little further change between one week and three weeks. Patients' three-week recognition of the word list was also impaired $(\mathrm{p}=0.012)$. Patients and controls were indistinguishable on 30-minute recall of designs, but there was an increasing separation between the groups at one week and three weeks $(\mathrm{p}=0.04)$. There was no significant difference between the groups in design recognition at three weeks $(p=0.745)$. Patients recalled significantly fewer elements of the story at all time intervals compared with control subjects, and threeweek recognition was impaired $(\mathrm{p}=0.025)$. Repeated measures ANOVA revealed an interaction effect of delay with group for the word list $(\mathrm{F}(1.2,46)=16.9, \mathrm{p}<0.001)$, designs $(\mathrm{F}(1.6,41)=4.3, \mathrm{p}=0.030)$ and story $(\mathrm{F}(1.9,45)=10.5, \mathrm{p}<0.001)$ indicating that patients' recall was differentially poorer than that of controls over the three-week delay for both verbal and visual material. Separate analyses were performed comparing controls with patients who did (AF+) and did not (AF-) have subjective complaints of accelerated forgetting. For AF- patients, no delay by group interaction was found for the word list $(\mathrm{F}(2.1,33)=1.9, \mathrm{p}=0.150)$, the designs $(\mathrm{F}(1.67,31)=$ $0.27, \mathrm{p}=0.730)$ or the story $(\mathrm{F}(2.1,33)=1.9, \mathrm{p}=0.151)$. For $\mathrm{AF}+$ patients, however, there was a highly significant delay by group interaction for words $(F(2.2,33)=19.7$, $\mathrm{p}<0.001)$, designs $(\mathrm{F}(1.8,31)=21.5, \mathrm{p}<0.001)$ and story $(\mathrm{F}(2.2,33)=19.7, \mathrm{p}<$ 0.001). AF+ and AF- patients did not differ from each other on any of the standard neuropsychological measures. No patients had seizures or auras between testing sessions. Within the patient group, delayed memory performance (3-week recall) did not correlate with HADS depression scores for the word list $(\mathrm{p}=0.940)$, designs $(\mathrm{p}=$ $0.910)$ or story $(p=0.746)$. The degree of forgetting shown between 30 minutes and three weeks did not correlate with forgetting over the initial 30 minutes for either patients or control subjects (word list: patients $r=0.100, p=0.643$; controls $r=$ $0.280, p=0.184$ ) (designs: patients $r=0.099, p=0.679$; controls $r=0.381, p=$ 0.073 ) (story: patients $r=0.308, p=0.142$; controls $r=-0.119, p=0.587$ ). Long-term forgetting also did not correlate with performance on any standard memory test. 


\subsubsection{Autobiographical memory}

Figure 4.2 shows the mean scores on the MAMI by decade of life for the two groups.

a)

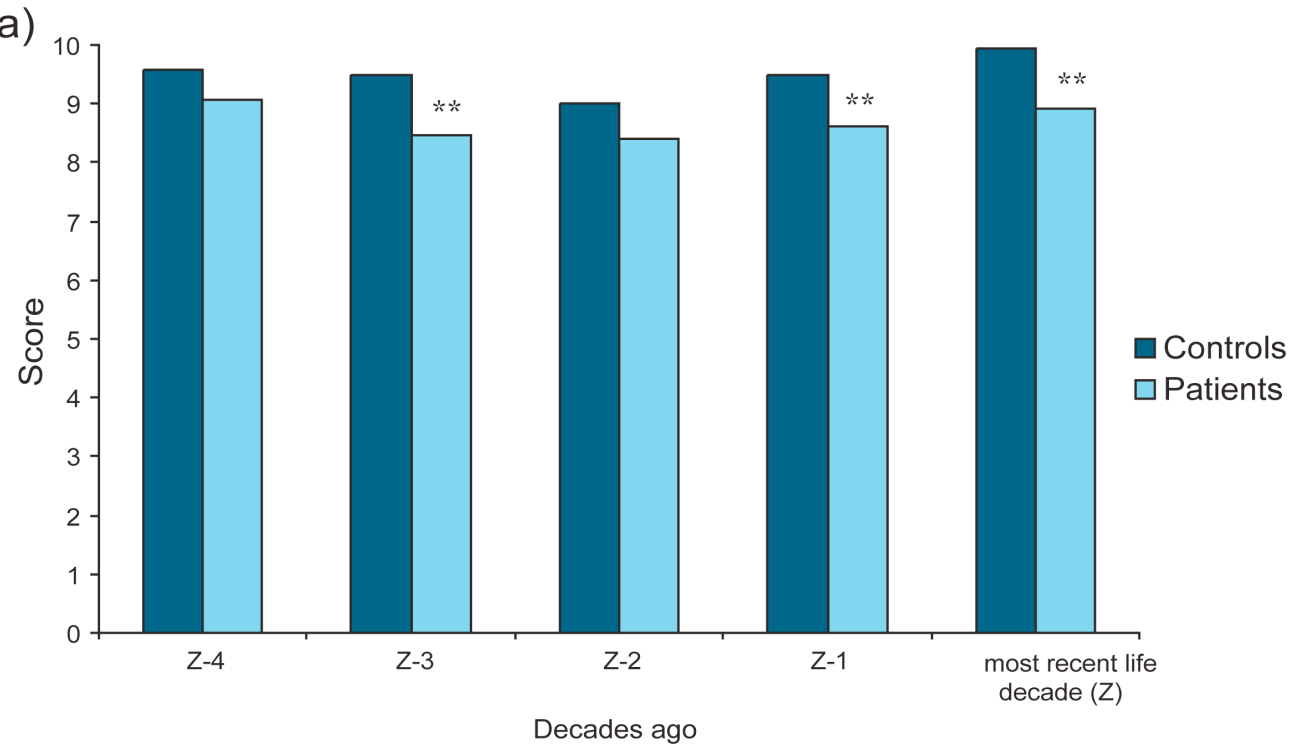

b) 10

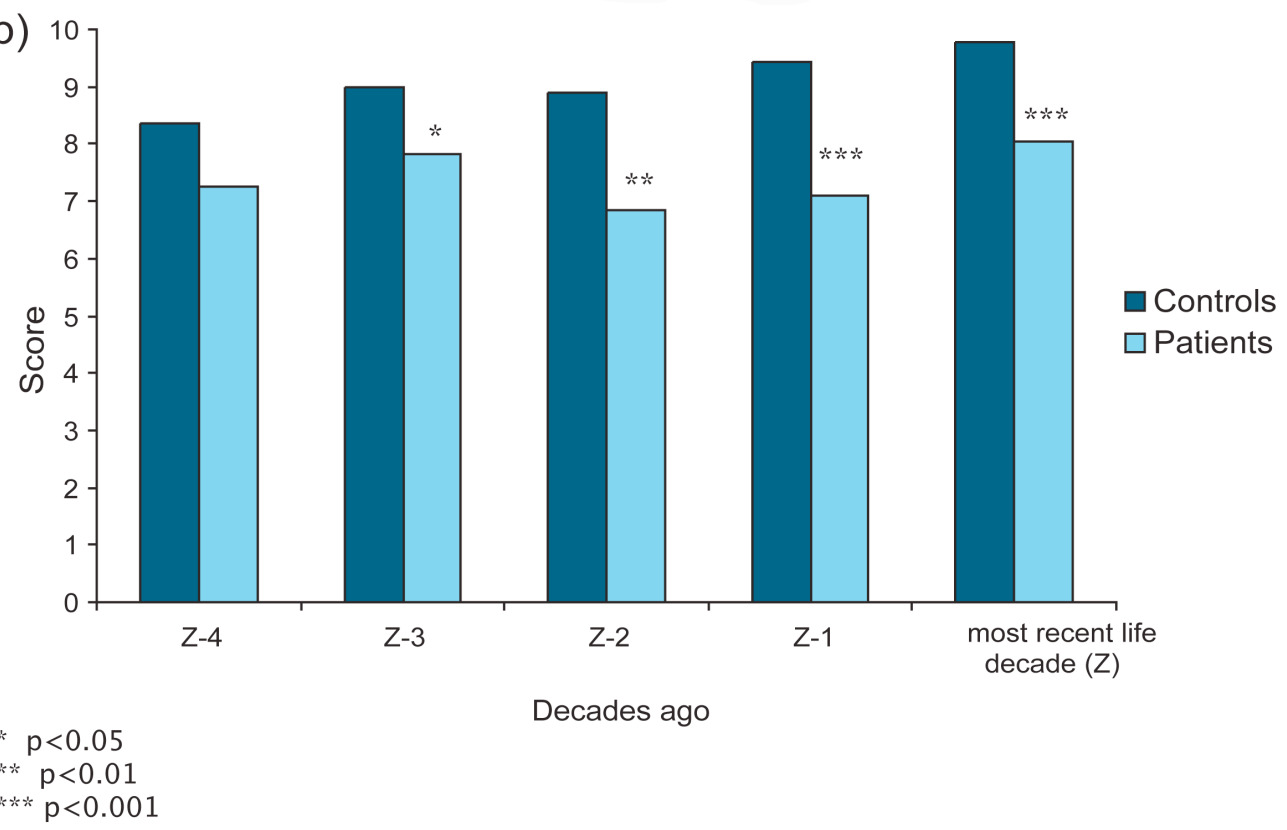

Figure 4.2: Mean scores of TEA patients $(n=22)$ and controls $(n=18)$ on the (a) semantic and (b) episodic memory components of the Modified Autobiographical Memory Interview (MAMI). ${ }^{*} \mathrm{p}<0.05 ; * * \mathrm{p}<0.01 ; * * * \mathrm{p}<0.001$. 
Patients showed a slight but statistically significant impairment of personal semantic memory recall in three of the five most recent decades of life and clear impairment in episodic memory scores across the four most recent life decades. Repeated measures ANOVA for semantic memory scores revealed a main effect for group $(\mathrm{F}(1,38)=$ $13.3, \mathrm{p}=0.001)$ and for decade $(\mathrm{F}(3.1,38)=3.3, \mathrm{p}=0.02)$ but no group by decade interaction $(\mathrm{F}(3.1,38)=0.5, \mathrm{p}=0.67)$. Repeated measures ANOVA for episodic memory scores revealed a main effect for group $(F(1,38)=24.4, p<0.001)$ but no effect for decade $(\mathrm{F}(4,38)=1.48, \mathrm{p}=0.21)$ or group by decade interaction $(\mathrm{F}(4,38)=$ $0.81, \mathrm{p}=0.52)$.

No correlation was observed between patients' lifetime autobiographical episodic memory scores and their HADS depression rating $(\mathrm{p}=0.76)$. MAMI summary scores (MAMI-s and MAMI-e) did not correlate with any standard neuropsychological measure among either the patient or control group.

\subsubsection{Relationship between ALF and autobiographical memory}

There was no correlation observed between average long-term forgetting scores (zALF) and MAMI-s $(r=0.151, p=0.480)$ or MAMI-e $(r=0.245, p=0.249)$ in the patient group. Figure 4.3 shows zALF and MAMI-e (converted into a z score) for every patient in subgroup 1 . The chart reveals a group of six patients (in the bottom right quadrant) who have a normal zALF score and yet show impairment ( $>2$ standard deviations from the control group mean) in autobiographical episodic memory. Furthermore, two patients (top left quadrant) demonstrate normal performance on the MAMI but accelerated long-term forgetting.

\subsubsection{Subjective memory scores and their relation to objective measures}

Patients rated their memories significantly worse than controls on both the EMQ (patients: mean $=29.7(\mathrm{SD}=16.2)$; controls: mean $=13.8(\mathrm{SD}=9.7) ; \mathrm{p}<0.001)$ and the VTLMQ (patients: mean $=10.0(\mathrm{SD}=7.1)$; controls: mean $=2.4(\mathrm{SD}=2.9) ; \mathrm{p}<$ $0.001)$. 


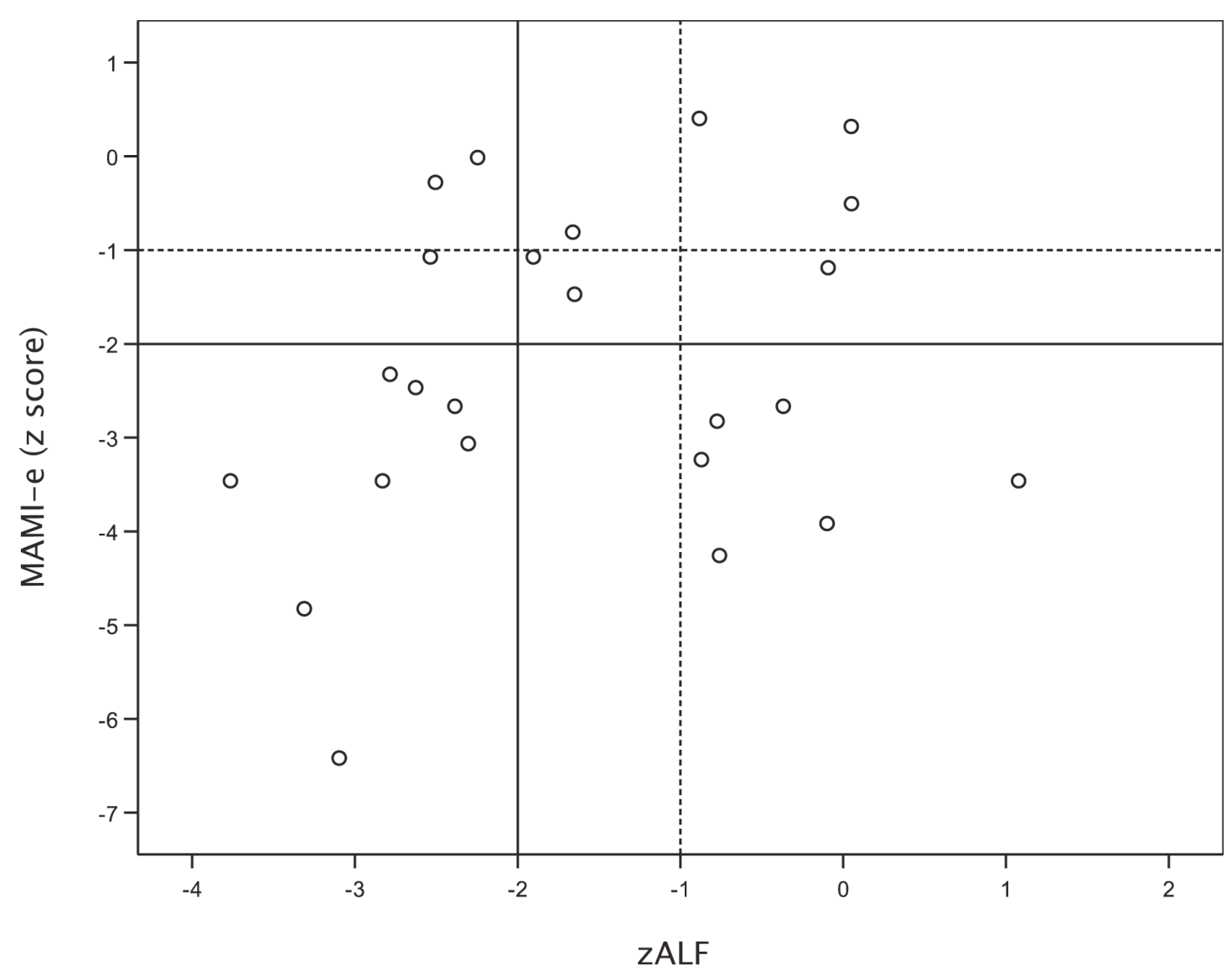

Figure 4.3: Average accelerated long-term forgetting scores (zALF) are plotted against average episodic autobiographical memory scores (MAMI-e (shown as z scores)).

Multiple linear regression analysis was used to investigate which demographic and neuropsychological factors were predictive of subjective memory scores. Separate analyses were conducted using EMQ and VTLMQ scores as dependent variables. The independent variables entered into the regression models were: age, sex, zmem, zALF, MAMI-e (highly correlated with MAMI-s and potentially more closely associated with subjective memory complaints), letter fluency (as a measure of executive function) and total HADS score. Only variables that met the forward selection criterion $(\mathrm{p}<0.05)$ were retained. The resulting models are shown in Table 4.2. 


\begin{tabular}{|c|c|c|c|c|}
\hline Independent variable & Dependent variable & $\boldsymbol{\beta}$ & $\mathbf{t}$ & $\mathbf{p}$ \\
\hline \multirow[t]{2}{*}{ EMQ } & HADS & 0.53 & 3.03 & 0.008 \\
\hline & zALF & -0.41 & -2.38 & 0.030 \\
\hline \multirow[t]{2}{*}{ VLTMQ } & HADS & 0.54 & 3.46 & 0.002 \\
\hline & MAMI-e & -0.35 & -2.24 & 0.036 \\
\hline \multicolumn{5}{|c|}{$\begin{array}{l}\text { EMQ = everyday memory questionnaire; HADS = Hospital Anxiety and Depression } \\
\text { Scale; MAMI-e = episodic component of the Modified Autobiographical Memory } \\
\text { Interview; VTLMQ = Very Long Term Memory Questionnaire; } \mathrm{ZALF}=\mathrm{Z} \text { score across } \\
\text { all long-term forgetting tasks }\end{array}$} \\
\hline
\end{tabular}

Age, sex, zmem and letter fluency were not predictive of either subjective memory measure. EMQ score was predicted by total HADS score $(\beta=0.53)$ and zALF $(\beta=-$ 0.41). The model was able to explain $49 \%$ of the variance in EMQ score. VTLMQ score was predicted by total HADS score $(\beta=0.54)$ and MAMI-e $(\beta=-0.35)$. The model was able to explain 51\% of the variance in VTLMQ score.

\subsection{Discussion}

In this chapter, I report the neuropsychological profile of 50 patients with TEA and a matched control group. The patient group as a whole were slightly impaired on standard anterograde memory tests compared with controls. However, a subgroup of patients with normal performance on these tests was shown to forget newly learned material more rapidly than controls over a three-week delay. Furthermore, patients in this group showed deficits in autobiographical memory for facts and events originating several decades prior to the onset of their seizures. Subjective memory measures correlated with these unusual forms of impairment rather than performance on standard neuropsychological tests.

The mean IQ of patients in this study was in the high average to superior range. This is likely to reflect selection bias: the diagnosis of TEA requires an accurate description of an unusual, transient disturbance of cognitive function. The TEA 
patient group as a whole was mildly impaired on tests of visual and verbal delayed recall and recognition memory. TEA is, therefore, associated with some decrease in interictal memory performance, but this is subtle. Performance on tests of language, semantic memory, visuospatial perception and executive function was unimpaired. This pattern is similar to that observed in patients with typical TLE, although more severe and widespread deficits are usually found in patients with an earlier onset of seizures, a greater seizure frequency and more antiepileptic medications (Motamedi and Meador, 2003). One interesting and as yet unexplained finding is the disproportionate impairment of TEA patients on the face recognition memory task. This observation was also made by Kapur (1993b). It is possible that this test is simply more sensitive than others to subtle memory impairment. Face-specific memory deficits are, however, well recognised and are often associated with damage to the fusiform gyrus. This region also activates preferentially to face stimuli in fMRI studies and has been called the fusiform face area (Kanwisher et al., 1997). It is possible, therefore, that TEA results in disproportionate dysfunction of networks important for remembering, and perhaps perceiving, faces. This needs to be explored in future work. It should be noted that the control group in the present study were largely nominated by patients in subgroup 1, patients who had been selected from the wider group for their apparently normal performance on the standard memory tests. It is possible that this procedure introduced bias towards better memory performance among the control group and therefore exaggerated the apparent impairment of the TEA group as a whole. This could have been avoided by having all 50 patients nominate a control subject. Depression ratings were marginally higher among patients than controls, in the absence of any difference in the rates of psychiatric diagnoses: this is unlikely to have influenced the results of cognitive testing, and did not correlate with objective memory performance at the individual level.

Subjectively reported memory difficulties are common and pronounced in TEA, as demonstrated by patients' responses to the EMQ and VTLMQ. These responses failed to correlate with any standard measures of anterograde memory, but were independently related both to mood and performance on novel tests that examined forgetting over an extended delay and remote autobiographical memory. Performance on standard memory measures has been shown to correlate poorly with subjective complaints in other types of epilepsy (Banos et al., 2004; Piazzini et al., 2001; 
Thompson and Corcoran, 1992), although this finding is not universal (Helmstaedter et al., 1998). The relationship between mood and subjective memory difficulties in epilepsy is widely recognised (Elixhauser et al., 1999; Piazzini et al., 2001). Our results suggest that, in TEA at least, ALF and autobiographical memory impairment may play an additional role. Nonetheless, considerable unexplained variance remains in our models, which may be attributable to several factors. The types of memory failure reported by patients, particularly in day-to-day memory, may reflect problems with attention and concentration rather than memory per se. Furthermore, the high mean IQ in this group may mean that patients are particularly aware of subtle changes in their memory abilities, especially when sensitised by ictal memory failure.

The subset of TEA patients in whom ALF was examined was unimpaired on standard neuropsychological tests and psychiatric measures. Learning rates were normal. However, over delays of 30 minutes to three weeks they showed abnormally rapid forgetting of material learned to a demanding criterion of $90 \%$ correct. Furthermore, those patients who had 'real-world' complaints of ALF showed significantly greater forgetting over the three-week interval than those who did not. The data thus bear out the patients' complaint of excessively rapid decay of recently acquired memories, and indicate that while this accelerated loss may be detected at 30 minutes it becomes more apparent over the following days to weeks. An important question is whether the subtle impairment at 30 minutes and the much more marked deficit observable after an extended delay are due to impairment in a single process or whether, as has been suggested by other authors, the results reflect a dissociation between early and late consolidation processes. Notably, the degree of forgetting observed over the extended delay did not correlate with forgetting over the initial 30 minutes or with scores on standard anterograde memory tests. Our findings are consistent with previous reports of accelerated forgetting among patients with temporal lobe epilepsy (Blake et al., 2000; Kapur et al., 1997; O'Connor et al., 1997). The cause of ALF is not yet clear, and a number of possibilities are discussed in detail in the following chapter. Clinical seizures had been abolished in all patients tested and are not, therefore, a necessary condition. High serum levels of antiepileptic drugs have been implicated in the reduced retention of information amongst patients with refractory epilepsy (Jokeit et al., 2001). However, in the present study, patients' memory complaints antedated the initiation of treatment and anticonvulsant doses were 
generally low. Our study design did not allow for pre- and post-treatment assessment of neuropsychological function, and further work is required to evaluate the relationships between ALF, clinical and subclinical epileptiform activity and any underlying structural pathology.

This study has also demonstrated impaired recall of autobiographical memories acquired many years before the onset of TEA. Once again, this bears out patients' subjective accounts of their memory difficulties. Further work is required to investigate whether other forms of remote memory, including memory for public events, facts, people and places, are equally impaired. The data also suggest a dissociation between ALF and remote autobiographical memory. Six patients showed no evidence of ALF but were markedly impaired on the MAMI. These patients (cases 8, 38, 83, 91, 93, 124 - see Table 2.1) all complained of autobiographical memory loss extending several decades into the past but experienced the onset of amnesic attacks in the past ten years in three cases (cases 91, 93, 124) and two years in the other three cases (cases 8, 38, 83). As discussed in Chapter 1, the occurrence of prominent retrograde amnesia in the presence of normal or near normal anterograde memory has been termed 'focal retrograde amnesia' (FRA). While the explanation is often 'psychogenic' (Kapur, 2000; Kopelman, 2000), FRA can be caused by a range of mechanisms in brain disease (Evans et al., 2003; Kapur et al., 1992; Markowitsch et al., 1993). Kopelman (Kopelman, 2000) has raised two objections to the use of the term 'focal retrograde amnesia' in the context of TEA. Firstly, he notes that the observed autobiographical memory deficit may not be truly focal because long-term anterograde memory might be impaired (i.e. ALF). The patients listed above appear to counter this objection, although it is conceivable that ALF appears only at delays longer than three weeks in some cases. The second objection is that seizure activity may have begun much earlier in the patient's life than the clinically apparent seizures and thus that their autobiographical memory deficit may reflect an anterograde rather than a retrograde deficit. Future studies should investigate this possibility using longitudinal measurements of autobiographical memory. However, reports from patients and their relatives suggest that previously well-established remote memories are progressively lost following the onset of the amnesic attacks. TEA appears, therefore, to be associated with a distinctive subtype of 'neurogenic' FRA, the mechanisms of which are yet to be elucidated. As discussed in Chapter 1, the neural 
basis of autobiographical memory is currently the subject of a lively debate between proponents of the 'standard theory' (Squire et al., 2004), which proposes that the medial temporal lobes play a temporary role in episodic memory, and 'multiple trace theory' (Moscovitch et al., 2005), which holds that the medial temporal lobes are required permanently for episodic memory recollection. In TEA, the co-occurrence of transient amnesic seizures, ALF and autobiographical amnesia in patients with a presumed seizure source in the medial temporal lobes lends some support to the multiple trace theory, and provides an opportunity to test the predictions of this hypothesis.

\subsection{Summary}

The work reported in this chapter explores the neuropsychological characteristics of TEA. The results show that patients with TEA rate their own memory function significantly worse than do control subjects. Standard tests of anterograde memory revealed subtle impairment at the group level. However, a subgroup of patients with normal performance on these standard tests demonstrated accelerated forgetting of new information over an extended delay of three weeks, as well as extensive impairment of autobiographical memory. Subjective memory ratings were unrelated to performance on standard neuropsychological tests but were partially explained by mood and by ALF and autobiographical memory scores. These two relatively novel phenomena therefore have a significant clinical impact on patients with TEA and may provide useful theoretical insight into human memory processes. It is possible that ALF and remote memory loss are found in patients with other forms of epilepsy. The following chapter presents the results of a comprehensive review of these phenomena in epilepsy, highlights methodological difficulties surrounding their investigation and discusses some of their clinical and theoretical implications. 
Chapter 4 


\section{CHAPTER FIVE}

\section{A comprehensive review of accelerated long-term forgetting and remote memory impairment in epilepsy}

\subsection{Introduction}

In the previous chapter, it was demonstrated that patients with TEA, who often complain of interictal memory problems, exhibit accelerated forgetting of newly acquired memories over a period of three weeks as well as deficits in remote autobiographical memory for events that occurred several decades prior to the clinical onset of their epilepsy. Moreover, these unusual difficulties may occur in the absence of any demonstrable impairment on standard tests of cognitive function and contribute to the patient's subjective assessment of their memory performance.

Accelerated long-term forgetting (ALF) is not a widely recognised phenomenon in clinical neuropsychology. As discussed in Chapter 1, it is often assumed that, once memories have been successfully encoded into long term memory, forgetting proceeds at a rate unmodulated by factors such as age, intelligence, material type, initial acquisition rate and various types of brain pathology. Investigations of longterm retention in patients with brain pathology are, therefore, relatively rare.

Impairment of remote memory, on the other hand, has been the focus of much research in recent years. Standard theories of long-term memory predict that damage to the medial temporal lobes will result in a temporally graded retrograde amnesia, with sparing of very remote memories. However, some researchers have emphasised cases in which retrograde amnesia is extensive and ungraded after very focal hippocampal injury, and have concluded that true episodic memories remain dependent upon the hippocampus for the duration of their existence. In either case, as discussed in Chapter 1, remote memory loss is generally expected to be accompanied by a deficit in anterograde memory. 
The existence of ALF and remote memory impairment (RMI) in TEA raises the possibility that these two phenomena may be exhibited more widely amongst patients with epilepsy. Furthermore, their role in partially explaining subjective impressions of memory impairment in TEA may also generalise to other forms of epilepsy, in which the mismatch between subjective and objective assessments of memory has often been noted but remains poorly understood. In this chapter, I report a comprehensive review of the literature on ALF and RMI in epilepsy. Results from the TIME Project are included in order to place them in this wider context and are referenced as Butler et al., 2007. I discuss evidence for the existence of each of the two phenomena; methodological issues surrounding their assessment; their possible pathophysiological mechanisms and their relevance to the theoretical understanding of human memory.

\subsection{Accelerated long-term forgetting}

According to traditional models, information has been encoded into long-term memory if it can be accurately retrieved after an interval during which active rehearsal is prevented. Thereafter, a process of 'consolidation' is thought to render the memory trace progressively less vulnerable to disruption (Squire et al., 1984). This process, thought to involve a gradual reorganisation of the memory trace at a neural systems level (Squire and Alvarez, 1995), may continue for weeks, months or even years. However, it is often assumed that its efficacy can be assessed at relatively brief delays. Standard neuropsychological instruments, therefore, typically test memory retention at intervals of up to 30 minutes, and little is known about forgetting beyond that point. Over recent years, a number of case reports have described a novel form of forgetting that is apparent only over extended periods of time and provides evidence for a prolonged multiple-stage consolidation process. These cases, together with group studies evaluating very long-term forgetting in epilepsy, are the focus of this section. Several of the studies included have been summarised in a recent review paper by Bell and Giovagnoli (2007). Although other authors have called this phenomenon long-term amnesia (LTA) (Kapur et al., 1997; Kapur et al., 1996b; Mayes et al., 2003), here the alternative accelerated long-term forgetting (ALF) is adopted to distinguish the disorder from the amnesic syndrome and to include cases in which long-term memory may be deficient but not completely absent. 


\subsubsection{Methods}

An exhaustive search of the medical and psychological literature was performed to identify case reports and group studies of ALF associated with epilepsy. The search covered publications indexed in MEDLINE (from 1966), EMBASE (from 1980) and PSYCHINFO (from 1967) prior to the end of November 2007. The titles and available abstracts of pertinent papers were scrutinised and further hand searching of reference lists was used to identify un-indexed reports. First, case reports were identified that described patients with epilepsy in whom learning and memory performance over delays of up to one hour was considered to be in the normal range (by comparison with published norms or a matched control group) but in whom testing over longer delays revealed impairment. Second, group studies were identified that compared memory over delays of 24 hours or longer in patients with epilepsy and control subjects.

\subsubsection{Case reports}

Seven case reports of ALF meeting the inclusion criteria were found (Cronel-Ohayon et al., 2006; Kapur et al., 1997; Kapur et al., 1996b; Lucchelli and Spinnler, 1998; Manning et al., 2006; Mayes et al., 2003; O'Connor et al., 1997). Three additional cases were described as showing ALF but did not undergo formal testing (Kapur et al., 1996b; Kapur et al., 1989) or were tested only at short delays (Maravita et al., 1995), and one report demonstrated abnormal long-term forgetting in a patient with no documented seizures (De Renzi and Lucchelli, 1993). The seven cases considered are summarised in Table 5.1. Four patients were male and three female. Their ages ranged from 18 years to 65 years $($ mean $=48.1, \mathrm{SD}=15.6)$. In striking contrast with classically amnesic individuals, these patients maintained active, independent lives and several remained in employment.

The aetiology of the memory impairment included: closed head injuries (Kapur et al., 1996b; Mayes et al., 2003), paraneoplastic limbic encephalitis (O'Connor et al., 1997), neuronal dysplasia in the left amygdala (Cronel-Ohayon et al., 2006) and hippocampal sclerosis (Manning et al., 2006). Two patients had late-onset temporal lobe epilepsy with no clear cause (Kapur et al., 1997; Lucchelli and Spinnler, 1998). In two cases (Kapur et al., 1996b; Lucchelli and Spinnler, 1998) seizures resolved immediately with anticonvulsant medication. 


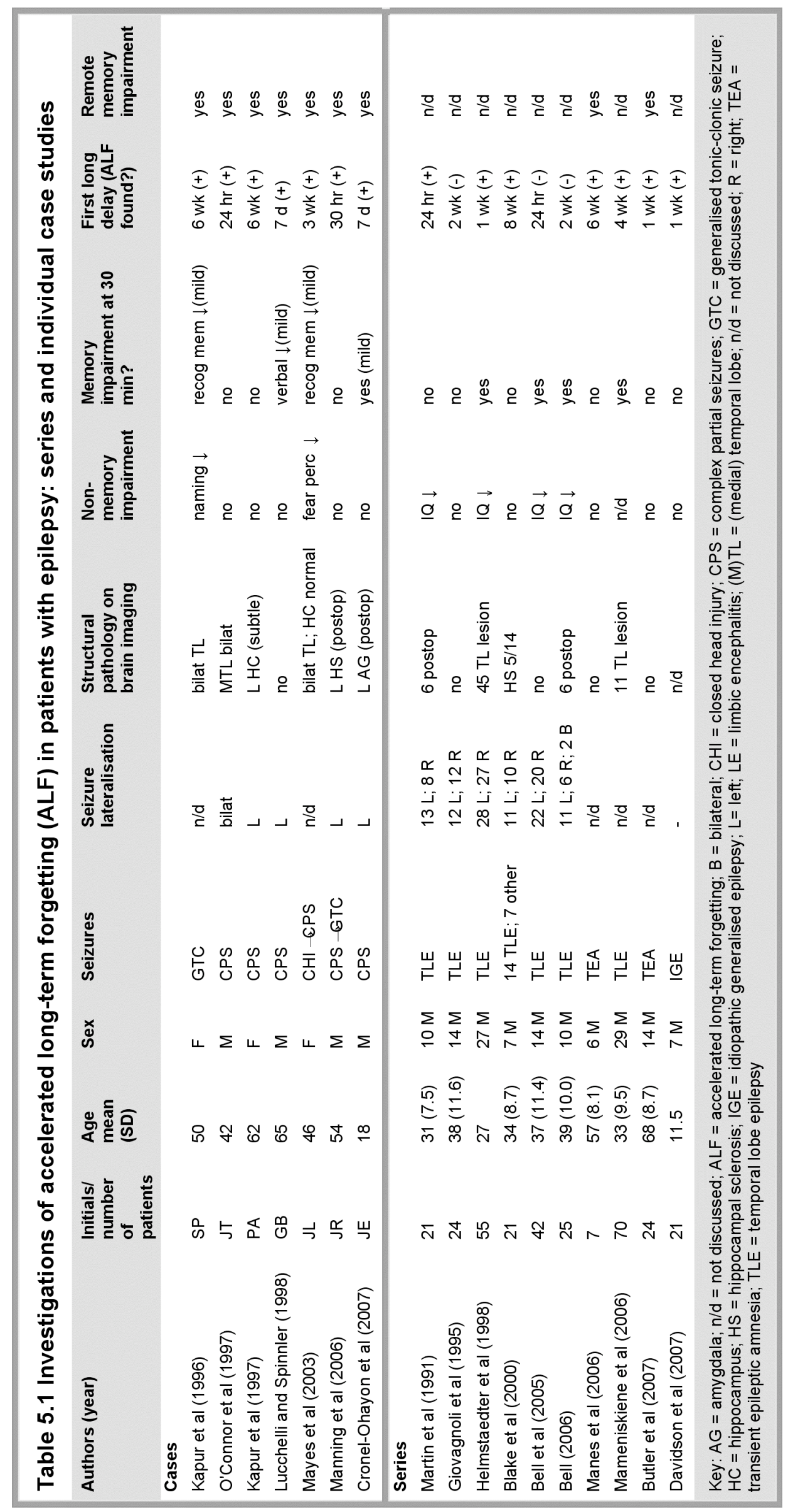


Structural brain imaging was abnormal in six patients. Damage was restricted to the temporal lobes in all cases except one (Mayes et al., 2003), in whom the right orbitofrontal cortex was also affected. The hippocampus was the only abnormal region in one case (Kapur et al., 1997), whereas this was spared in another (Mayes et al., 2003). Both medial and lateral temporal cortices were damaged in the remaining four cases (Cronel-Ohayon et al., 2006; Kapur et al., 1996b; Manning et al., 2006; O'Connor et al., 1997), two of whom had undergone temporal lobectomy (CronelOhayon et al., 2006; Manning et al., 2006). Only one patient had normal brain imaging (Lucchelli and Spinnler, 1998).

Reporting of standard neuropsychological test results is variable. Five cases had no apparent deficits in non-memory domains, whilst others showed mild impairment at naming (Kapur et al., 1996b) and fear perception (Mayes et al., 2003). In three cases, standard memory tests revealed no abnormality (Kapur et al., 1997; Manning et al., 2006; O'Connor et al., 1997) whilst, in the remainder, subtle deficits were found in recognition memory (Kapur et al., 1996b; Mayes et al., 2003) or recall (CronelOhayon et al., 2006; Lucchelli and Spinnler, 1998).

Very long-term memory was assessed using a variety of tests including a short story, word lists, verbal paired associates, the Rey complex figure and face recognition. Immediate and early (between 10 and 30 minutes) recall was within the range of control subjects in all patients for all types of material, except for story recall in two cases (Cronel-Ohayon et al., 2006; Manning et al., 2006). However, accelerated forgetting was found to have occurred at intervals ranging from 24 hours (Manning et al., 2006; O'Connor et al., 1997) to six weeks (Kapur et al., 1997; Kapur et al., 1996b). Recall at the longest delays was at floor in most cases, and several patients were unable even to remember the learning episode. Recognition memory was sometimes less impaired (Kapur et al., 1997; Kapur et al., 1996b; Manning et al., 2006; Mayes et al., 2003), but never completely intact. More precise study of the forgetting rate is made in four papers. O'Connor et al. (1997) described a rapid decline in memory over the first 24 hours, with subsequent decline to floor over seven days. Lucchelli and Spinnler (1998) showed their patient to have good verbal recall at 24 hours but to score at floor after seven days. The patient of Manning et al. (2006) exhibited poor story recall and relatively preserved face recognition at 30 hours, but 
was severely impaired on both tests after seven days. Cronel-Ohayon et al. (2006) found accelerated forgetting after seven days, and little subsequent change after 29 days.

Only two patients had documented seizures during the retention interval (Mayes et al., 2003; O'Connor et al., 1997). O'Connor et al. (1997) assessed forgetting before and during anticonvulsant therapy, and found an improvement in very-long term memory once seizures were controlled.

Accelerated long-term forgetting was accompanied in all cases by impairment of remote memory. In all but two cases (Kapur et al., 1997; Manning et al., 2006), there was a profound loss of autobiographical memories from across the lifespan, not merely from the period since the apparent onset of anterograde memory problems. Memory for public events dating from the premorbid period was affected in every case.

\subsubsection{Group studies}

Ten studies addressing the issue of long-term anterograde memory in epilepsy were identified (Bell, 2006; Bell et al., 2005; Blake et al., 2000; Butler et al., 2007; Davidson et al., 2007; Giovagnoli et al., 1995; Helmstaedter et al., 1998; Mameniskiene et al., 2006; Manes et al., 2005; Martin et al., 1991). These are summarised in Table 5.1. Nine examined patients with TLE, and one of these (Blake et al., 2000) also included a group of seven patients with other focal epilepsies. Two studies (Butler et al., 2007; Manes et al., 2005) exclusively investigated TEA. The mean patient age ranged from 27 to 39 years, except for TEA patients who were older. One study investigated children with idiopathic generalised epilepsy (IGE) (Davidson et al., 2007), in whom the mean age was 11.5 years (range 8 to 16). In total, 138 male and 172 female patients were studied.

The aetiology of the epilepsy in patients included in these studies is less explicitly discussed than in the case reports but, in contrast to several of the cases, did not generally involve widespread brain injury. Neuroimaging revealed varying proportions of hippocampal sclerosis, neoplastic and other lesions among patients, but 
no study quantitatively assessed their relation to memory function. Post-operative patients were included in the studies by Martin et al. (1988) (6 patients) and Bell (2006) (6 patients). The TEA patients studied by Manes et al. (2005) and Butler et al. (2007) had no clinically apparent abnormalities on brain MRI besides, in some cases, evidence of vascular disease. Davidson et al. (2007) do not report imaging findings, but these are expected to be normal in IGE.

Memory retention over 30 minutes was found to be comparable in patients and controls in five studies (Blake et al., 2000; Butler et al., 2007; Davidson et al., 2007; Giovagnoli et al., 1995; Martin et al., 1991), and one (Manes et al., 2005) found normal verbal but impaired non-verbal memory. Four studies (Bell, 2006; Bell et al., 2005; Helmstaedter et al., 1998; Mameniskiene et al., 2006) identified impairment amongst patients even at this short delay. At extended intervals ranging from 24 hours (Bell et al., 2005; Martin et al., 1991) to eight weeks (Blake et al., 2000), seven studies (Blake et al., 2000; Butler et al., 2007; Davidson et al., 2007; Helmstaedter et al., 1998; Mameniskiene et al., 2006; Manes et al., 2005; Martin et al., 1991) found patients to show a disproportionate degree of forgetting compared with controls. In contrast, three studies (Bell, 2006; Bell et al., 2005; Giovagnoli et al., 1995) found no difference in long-term forgetting rate between patients and controls. Bell et al. (2005) and Bell (2006) also failed to identify a significant number of individual patients in whom memory performance was normal at 30 minutes but impaired at an extended delay. Only one study (Butler et al., 2008) examined remote memory and found extensive deficits in patients with TEA.

The inconsistency among these results raises questions about the existence, scope and prevalence of ALF amongst patients with epilepsy, the best means of assessing very long-term memory, the pathophysiology of ALF, and its relation to RMI. 


\subsubsection{Discussion}

\subsubsection{Does ALF occur in epilepsy? If so, for which types of material and is there a laterality effect?}

The seven case reports suggest that ALF is an identifiable clinical phenomenon closely linked to epilepsy. Several of the group studies identified ALF in patients with TLE and one in patients with IGE. However, three studies report negative results. The reason for this discrepancy is likely to arise partly from the methodological differences discussed below. ALF may also be more prominent in certain subtypes of TLE, such as TEA, in which $44 \%$ patients report symptoms consistent with ALF (Butler et al., 2007).

Patients were shown to learn at a normal rate in three studies (Blake et al., 2000; Butler et al., 2007; Martin et al., 1991), whereas learning was found to be impaired by Helmstaedter et al. (1998), Giovagnoli et al (1995) and Davidson et al (2007). In the latter study, the learning impairment shown by IGE patients accounted for most of the difference in very long-term forgetting, suggesting that an encoding problem may underlie the ALF seen in IGE.

The available data are inconclusive on the issue of whether hemispheric differences cause material-specificity in ALF. Using a test of verbal memory, Blake et al. (2000) identified ALF in patients with left-sided but not those with right-sided TLE. On the other hand, Martin et al. (1991), again only using verbal material, found no hemispheric effect. Without identifying the seizure focus, two studies (Butler et al., 2007; Mameniskiene et al., 2006) found ALF for both verbal and non-verbal material. Amongst IGE patients, ALF was identified for verbal material and a trend towards ALF for non-verbal material.

The balance of evidence suggests that recognition memory is also affected by ALF, but the findings are inconsistent. Six studies (Bell, 2006; Bell et al., 2005; Blake et al., 2000; Butler et al., 2007; Helmstaedter et al., 1998; Manes et al., 2005) found patients to show impairment on recognition memory at extended delays. Martin et al. (1991), on the other hand, did not find recognition memory impairment, although their delay was just 24 hours. Davidson et al. (2007) also found no recognition memory deficit 
amongst IGE patients, even after a 7-day delay, although patients and controls scored close to ceiling.

In summary, the data currently available suggest that, in some patients with epilepsy, anterograde memory performance is normal at standard test intervals but declines abnormally rapidly thereafter - the phenomenon of ALF. The negative results indicate that this does not always occur. Some possible explanations for these mixed results are considered below.

\subsubsection{Methodological issues in the assessment of ALF}

The assessment of long-term forgetting encounters a number of methodological problems:

i) Patient and control groups should be matched as carefully as possible for demographic and non-memory cognitive variables to isolate the phenomenon of interest. Thus it may be relevant that in the studies by Martin et al. (1991), Helmstaedter et al. (1998), Bell (2006) and Bell et al. (2005) patients had a significantly lower mean IQ than control subjects. Other groups (Blake et al., 2000; Butler et al., 2007; Manes et al., 2005) performed more extensive testing and revealed no differences between patients and controls on measures of language, visuospatial perception and executive function.

ii) ALF is most convincingly demonstrated when patients exhibit normal initial learning and 30 minute recall, but clear impairment at longer delays (Blake et al., 2000; Butler et al., 2007; Manes et al., 2005; Martin et al., 1988). In cases where patients are already impaired over short delays, a number of techniques may be used to assess long-term forgetting rates:

a. Using a variety of the technique introduced by Huppert and Piercy (1978), the experimenter can modulate exposure to the study material to ensure that patients and control subjects reach the same initial level of learning. In four studies (Blake et al., 2000; Butler et al., 2007; Davidson et al., 2007; Martin et al., 1988), all the material was repeatedly presented until the subject reached a learning criterion. As Bell (2005) has observed, this 'over-learning' method may mask early 
forgetting with a ceiling effect. However, in the studies by Blake et al. (2000) and Butler et al. (2007), patients who showed ALF of 'overlearned' material nonetheless performed normally on standard memory tests. An alternative, employed by four studies (Bell, 2006; Bell et al., 2005; Giovagnoli et al., 1995; Martin et al., 1988), is to use a 'selective reminding' technique, in which only non-remembered items are represented at each learning trial.

b. Individual patients and controls may be matched for learning on a case-by-case basis. This method, however, risks producing nonrepresentative results if 'upper range' patients are matched with 'lower range' control subjects.

c. Differing acquisition levels may be accepted and the overall shape of the forgetting curves compared. This method was employed by three studies (Bell, 2006; Bell et al., 2005; Mameniskiene et al., 2006), with mixed results. The problem which arises here is that there is no widely accepted model of how variations in initial learning level affect forgetting over time (Rubin and Wenzel, 1996).

iii) The choice of study material is likely to be important. Relative impairments in verbal and non-verbal memory depend, in other contexts, on the laterality of the seizure focus. Also, forgetting may be different for semantically related (e.g. a story) and unrelated (e.g. a word list) material (Isaac and Mayes, 1999a; Isaac and Mayes, 1999b).

iv) Rehearsal of the material between test sessions may confound results. In some studies, subjects were forewarned about the delayed tests whereas in others they were not. The material used will also influence this: a story is more likely to be rehearsed than a large number of meaningless visual designs.

v) The length of the interval between testing sessions may determine whether or not ALF is found - the underlying mechanisms may operate over 24 hours or several weeks. An interval should be chosen at which control subjects perform neither at ceiling nor floor.

vi) The nature of the retrieval task - free recall, cued recall or recognition may be important. Davidson et al. (2007) suggest that their failure to find a deficit in recognition memory at an extended delay implies that ALF in 
their patients was due to a problem with memory retrieval rather than storage.

vii) When the same material is probed at several time intervals, memories are presumably reencoded to some degree at each probe. This in itself is likely to alter the time course of forgetting. One way of avoiding this problem is to test distinct subsets of the originally learned material at each interval.

\subsubsection{Aetiology and pathophysiology of ALF}

Accepting that ALF occurs in some patients with epilepsy, several contributory mechanisms may be hypothesised: 1) clinical or subclinical seizure activity; 2) structural brain pathology; 3) an adverse effect of anticonvulsant medication or 4) psychological mechanisms.

\section{Seizures}

Anecdotally, patients with TEA sometimes report that seizures seem to 'wipe out' memories of preceding events, and feel that their memory abilities improve once seizures are controlled with anticonvulsant therapy. O'Connor et al. (1997) document such an improvement in a single case of temporal lobe epilepsy. Mameniskiene et al. (2006) found a positive correlation between long-term forgetting and both i) manifest seizures during the experimental period and ii) subclinical epileptiform EEG activity. As in the study by Blake et al. (2000), however, long-term memory was not found to correlate with the average seizure frequency reported by patients. The close association between amnesic attacks and waking in TEA raises the intriguing question of whether sub-clinical, nocturnal epileptiform activity might disturb sleep-dependent memory consolidation processes (Ellenbogen et al., 2006; Walker, 2005; Walker and Stickgold, 2006).

In a study directly addressing the question of whether incident seizures accelerate forgetting, Bergin et al. (1995) tested immediate, 30 minute and 48 hour memory for verbal and non-verbal material in 58 patients undergoing video telemetry for the investigation of medically refractory partial seizures. No difference was found in long-term forgetting between patients who did and did not have seizures during the study period. This important result does not, however, rule out a negative influence of seizures upon anterograde memory. Features such as the timing, duration and 
anatomical focus of seizures may play an important role. Jokeit et al. (2001) examined memory over 24 hours for verbal material in a small group of patients $(n=10)$ undergoing videotelemetry. They found a difference in long-term recall between days with and without seizures, but this was restricted to the group of patients with a left temporal lobe seizure focus. A further source of evidence comes from studies that document an improvement in verbal memory scores in patients following right temporal lobectomy (Martin et al., 1998; Novelly et al., 1984). This finding suggests that a seizure focus in one hippocampus can negatively affect function in distant brain regions.

The question of whether transient impairment of neuronal function can disrupt very long-term memory has also been addressed in patients undergoing electroconvulsive therapy (ECT) for depression, a procedure known to induce anterograde and retrograde amnesia. Squire (1981) investigated recognition memory for pictures and sentences at intervals of 10 minutes, 30 minutes and 30 hours in patients on two occasions: two hours and four months after ECT. The subjects therefore acted as their own controls. Initial acquisition was matched by using longer stimulus presentation on the earlier occasion. Picture forgetting was significantly more rapid when the subjects had recently received ECT. On the other hand, patients with Korsakoff's syndrome (diencephalic amnesia) and a patient with chronic medial temporal lobe amnesia did not show accelerated forgetting when initial acquisition was matched to a group of healthy control subjects. These findings were replicated and extended by Lewis and Kopelman (1998) who included a group of depressed patients not undergoing ECT. Accelerated forgetting was again found solely in the post-ECT group and could therefore not be attributed to depression per se. Transient impairment of brain function also underlies post-traumatic amnesia (PTA). Levin et al. (1988), investigating recognition memory for photographs, found accelerated forgetting over 32 hours in head injury patients in PTA, compared with head injury patients who had recovered from PTA.

\section{Structural lesions}

Many of the case studies reviewed above also had radiological evidence of structural brain pathology involving the medial temporal lobes, although this is certainly not always evident in patients with ALF. The phenomenon might, therefore, represent a 
mild form of the amnesic syndrome, caused by subtle damage to the medial temporal lobes.

If so, forgetting should be dramatically accelerated in amnesic patients. A number of studies have addressed this issue and results have been mixed (see Isaac and Mayes (1999a) for a review). The problems of matching initial acquisition are of course much more acute here than in patients with normal or near-normal immediate memory. One early investigation suggested that accelerated forgetting was a feature of amnesia caused by medial temporal lobe lesions but not diencephalic lesions (Huppert and Piercy, 1979). However, this finding was later found not to be replicable (Freed et al., 1987). Isaac and Mayes (Isaac and Mayes, 1999a; Isaac and Mayes, $1999 \mathrm{~b}$ ) conclude that forgetting in the amnesic syndrome is accelerated over the first 10 minutes but only for certain types of material - specifically free recall of prose and semantically-related words. They interpret this as reflecting impairment of early consolidation processes due to medial temporal lobe damage. Beyond 10 minutes, forgetting rates have been measured in patients with anoxic brain damage (McKee and Squire, 1992), Alzheimer's Disease (Kopelman, 1985) and head injury after recovery from post-traumatic amnesia (Levin et al., 1988), and have been found to be normal. Accelerated forgetting over longer periods has been reported in healthy older subjects by some authors (Davis et al., 2003; Huppert and Kopelman, 1989), but not others (Petersen et al., 1992).

If ALF is essentially due to a mild form of hippocampal amnesia one might also predict some mild degree of impairment over standard testing intervals. The observation that some patients perform normally on standard tests yet exhibit ALF appears to argue against the existence of any defect in acquisition and initial retention of declarative memories. It could be, however, that standard tests are insufficiently sensitive to reveal a mild deficit in these stages of memory processing. Techniques such as recording event related EEG potentials at memory encoding could shed further light on this possibility.

Finally, additional imaging techniques, including manual or automated volumetric measurement, MR spectroscopy and diffusion tensor imaging have the potential to reveal subtle regional pathology within or affecting the MTL that may elude less sophisticated studies. In Chapter 6, I report the results of a detailed volumetric 
analysis of the MR images from the TIME Project and explore the relationships between regional brain volumes and memory performance.

\section{Anticonvulsant medication}

There is good evidence that antiepileptic drugs (AEDs) can have a negative impact upon cognition, although the field is fraught with methodological difficulties (Kwan and Brodie, 2001; Motamedi and Meador, 2004). The most commonly observed effects are slowed mental processing and reduced attention, and these are most marked with high doses and polytherapy. However, a specific impact on memory has been reported in several studies. Some newer drugs may have a better cognitive profile (Motamedi and Meador, 2003), although topiramate is possibly a significant exception (Thompson et al., 2000). The specific question of whether anticonvulsants can accelerate shorter term forgetting has been addressed in a single, retrospective study (Jokeit et al., 2005). Amongst 162 patients with medically refractory epilepsy, higher serum levels of AED were associated with greater forgetting of both verbal and visual material over a 30-minute delay after controlling for potentially confounding variables such as IQ, age, duration of epilepsy and seizure frequency.

Whilst it remains possible that the ALF observed in some studies reviewed above is a direct result of treatment with anticonvulsants, it seems unlikely to be the sole cause for a number of reasons: first, patients with TEA complain of ALF prior to initiation of therapy; second, patients with TEA usually report that their memory improves once treatment is started (Butler et al., 2007; Zeman et al., 1998); third, the forgetting observed by Blake et al. (2000) was specific to the group of patients with left temporal lobe epilepsy; and fourth, the doses of anticonvulsants used in TEA patients, those who complain most profoundly of ALF, are generally low.

\section{Psychosocial factors}

The disparity between subjective reports of memory difficulty amongst patients with epilepsy and their performance on neuropsychological tests (Corcoran and Thompson, 1992) has been attributed to disturbances of mood and poor self-esteem (Elixhauser et al., 1999; Giovagnoli et al., 1997). It is undoubtedly important to take such factors into account when investigating cognitive function in epilepsy. However, they are unlikely to play a major causal role in ALF. Three studies (Blake et al., 2000; Butler 
et al., 2007; Mameniskiene et al., 2006) assessed mood using the Hospital Anxiety and Depression Scale and found no correlation with very long-term memory performance. In addition, as mentioned above, Lewis and Kopelman (1998) did not find accelerated forgetting in a group of depressed patients after equating initial levels of learning.

\subsection{Remote memory in epilepsy}

Although anterograde memory deficits, at least over brief retention intervals, are well recognised in TLE, comparatively little is known about the integrity or otherwise of remote memory. However, RMI can have profound effects on an individual's life and may, on occasions, be the presenting complaint of patients with TLE (Gallassi, 2006; Gallassi et al., 1992). It is important, therefore, to ascertain the extent, aetiology and anatomical basis of any RMI as well as to explore potential therapeutic measures. From a theoretical perspective, investigation of the neuropsychological features of RMI will shed light on the way in which long-term memories are organised.

Remote memory is multifaceted. Broadly, the term encompasses memories that were encoded in the distant past. The temporal boundary at which recent memories become remote is vague but remote memories are usually thought of as being at least one year old. Research has concentrated on the declarative components of remote memory, which fall into several categories. Episodic memories relate to personally experienced events and are part of an individual's broader autobiographical memory, which also includes knowledge about 'personal semantics' such as where they went to school and what their first job was. Autobiographical memory can be distinguished from general knowledge - memory for public events, famous people, words and so forth. Deficits in remote memory may be due in part to a long-standing anterograde amnesia whereby neurological damage has impaired memory accumulation. However, there is evidence that retrograde amnesia, with loss of, or loss of access to, previously wellestablished memories can also occur, albeit rarely, in the absence of any anterograde impairment. As discussed in Chapter 1, reports of neurological disease causing such 'focal retrograde amnesia' have generated considerable debate (Kapur, 1993a; Kapur, 2000; Kopelman, 2000). If brain regions involved in the storage or retrieval of old memories are damaged, it is difficult - though not necessarily impossible (Evans et 
al., 2003) - to explain how newly acquired information could be successfully remembered over the long term. Moreover, a disproportionate affliction of retrograde memory is characteristic of psychogenic amnesia (Kopelman, 2002a), making a careful neuropsychiatric assessment mandatory in such cases. Examples of apparently focal retrograde amnesia have been associated with a range of aetiologies including traumatic brain injury (Levine et al., 1998), herpes simplex encephalitis (Tanaka et al., 1999), cerebral vasculitis (Evans et al., 2003), stroke (Miller et al., 2001) and TLE (Manes et al., 2001).

In this section, the evidence that TLE may be associated with remote memory deficits is reviewed by an examination of individual case reports and larger series

\subsubsection{Methods}

Using the methods described in section 5.2.1, a literature search was conducted for studies of remote memory in patients with epilepsy. In addition, I examined all reported cases of focal or disproportionate retrograde amnesia in which seizures were also a feature. As in section 5.2, the case studies are discussed first, as a demonstration that RMI can be associated with epilepsy, and then group studies are reviewed to investigate whether it occurs more generally.

\subsubsection{Case reports}

Eighteen case reports were found describing pronounced RMI in the context of epilepsy. In several, including four of the seven cases of ALF described above, seizures resulted from extensive brain damage caused by trauma or encephalitis. In this chapter, consideration is restricted to seven cases in six papers (Chan et al., 2007; Kapur et al., 1997; Kapur et al., 1989; Lucchelli and Spinnler, 1998; Manes et al., 2001; Manning et al., 2005) in which structural imaging was either normal or revealed only very focal abnormalities, since it is well recognised that structural pathology in a variety of brain regions can cause retrograde amnesia in the absence of seizures (Kopelman, 2002c). There were six males and one female patient. The ages ranged from 52 to 74 years. The details are summarised in Table 5.2. 


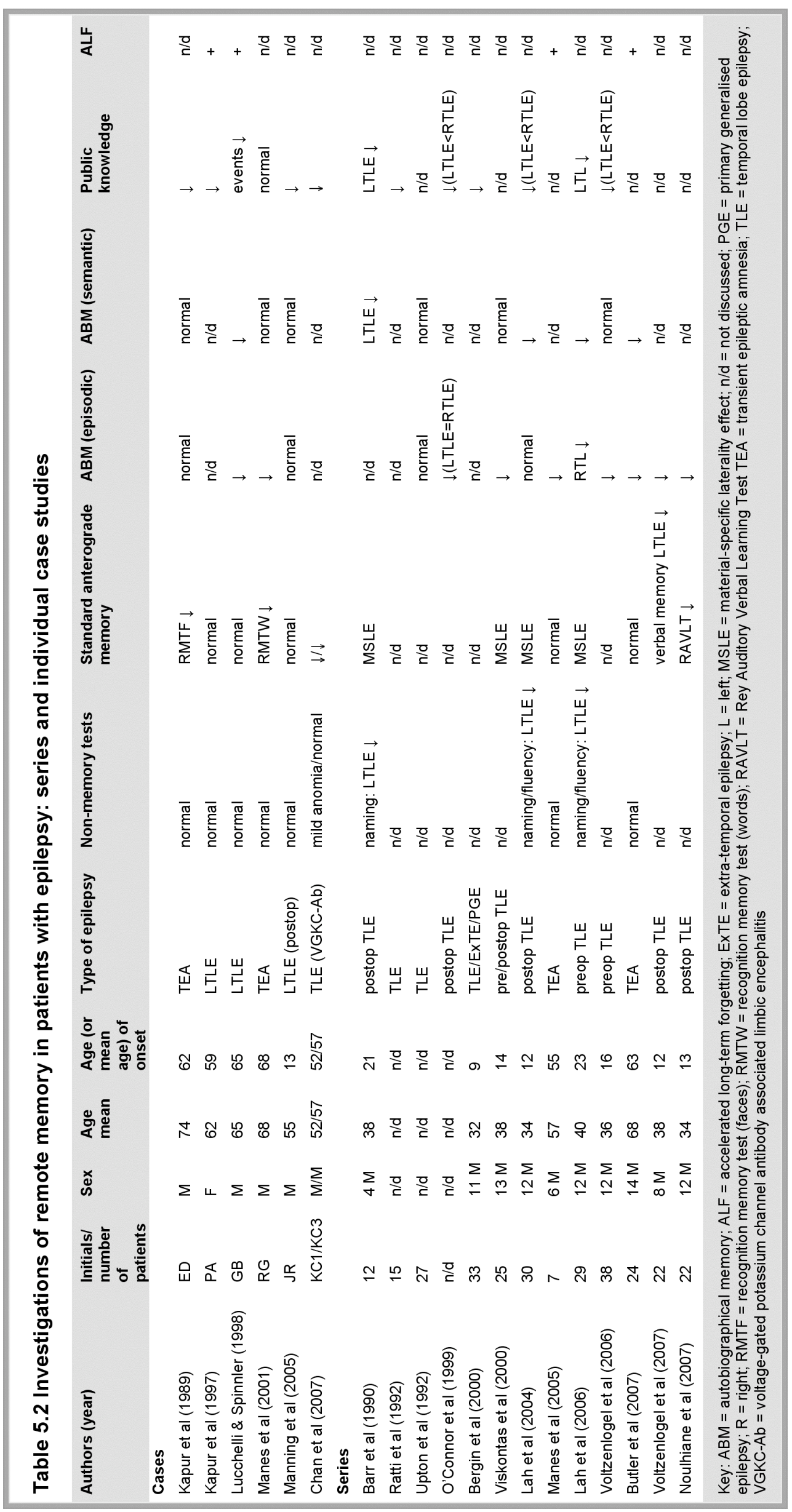


All seven patients had mesial temporal lobe epilepsy. This arose clearly from the left hemisphere in four cases, and was probably bilateral in three cases (Chan et al., 2007; Manes et al., 2001). One (Manning et al., 2005) had undergone a recent left temporal lobectomy for hippocampal sclerosis. Two cases had nonparaneoplastic limbic encephalitis associated with antibodies against voltage-gated potassium channels (VGKC-Ab) and bilateral medial temporal lobe damage seen on MRI (Chan et al., 2007). In the other four cases, seizures began in later life (range 58 to 69 years), there was no clear cause and neuroimaging was unremarkable except for a subtle left hippocampal lesion seen in one patient (Kapur et al., 1997). Two patients had TEA. Seizures were well controlled on medication in all cases.

General neuropsychological assessment was normal in most patients except for subtle recognition memory impairment in two cases (Kapur et al., 1989; Manes et al., 2001), and more general anterograde memory impairment in the two cases of VGKC-Ab encephalitis (Chan et al., 2007). Very long-term memory tests revealed ALF in two patients (Kapur et al., 1997; Lucchelli and Spinnler, 1998). The relative disturbance of autobiographical and public memory varied between cases. Public semantic memory appeared disproportionately impaired in two cases (Kapur et al., 1989; Manning et al., 2005), whereas detailed testing revealed disturbance only in the autobiographical domain in one case (Manes et al., 2001). In six cases, the deficit was shown to affect memories from the previous 30 to 40 years (Chan et al., 2007; Kapur et al., 1989; Lucchelli and Spinnler, 1998; Manes et al., 2001; Manning et al., 2005). In the other, it extended back just five years, and is interpreted by the authors as an anterograde memory problem caused by accelerated forgetting (Kapur et al., 1997).

\subsubsection{Group studies}

Thirteen studies were found that investigated remote memory in groups of patients with epilepsy (Barr et al., 1990; Bergin et al., 2000; Butler et al., 2007; Lah et al., 2004; Lah et al., 2006; Manes et al., 2005; Noulhiane et al., 2007; O’Connor et al., 1999; Ratti et al., 1992; Upton et al., 1992; Viskontas et al., 2000; Voltzenlogel et al., 2007; Voltzenlogel et al., 2006). The details are summarised in Table 5.2. 
The patient population varies across these studies. As discussed below, these differences have major implications for the interpretation of the resulting data. The only study to include patients with extratemporal or primary generalised epilepsy is that by Bergin et al. (2000), and these patients were found not to differ from controls on memory for remote public events. Five studies examined only patients who had undergone unilateral temporal lobectomy (Barr et al., 1990; Lah et al., 2004; Noulhiane et al., 2007; O'Connor et al., 1999; Voltzenlogel et al., 2007), whilst Viskontas et al. (2000) had a patient group consisting of both pre- and post-operative patients. In two further studies (Lah et al., 2006; Voltzenlogel et al., 2006), the patients investigated were candidates for surgery. There is therefore an emphasis towards the study of patients with medically intractable seizures. Interestingly, in one study no significant differences in remote autobiographical memory were found between the pre- and post-operative patients (Viskontas et al., 2000) and, in another, surgery was found to improve memory for recent events (Voltzenlogel et al., 2007). Of the remaining five studies, two (Butler et al., 2007; Manes et al., 2005) concentrated upon TEA, in which remote memory complaints are common. In total, over 262 patients were studied, and the mean age was approximately 42 years.

The detail in which the performance of patients and their age and sex matched control subjects on standard neuropsychological tests is reported varies considerably across the studies. Non-memory deficits are reported in two studies (Lah et al., 2004; Lah et al., 2006), in which patients with left TLE were impaired on verbal fluency and naming. With regard to anterograde memory, material-specific impairments were found amongst patients depending upon the side of their seizure focus in five studies (Barr et al., 1990; Lah et al., 2004; Lah et al., 2006; Noulhiane et al., 2007; Viskontas et al., 2002).

Twelve of the 13 studies found deficits in some form of remote memory, whether in episodic memory, personal semantics or public knowledge, amongst TLE patients. The results raise several questions about RMI in epilepsy including its prevalence and extent, the best methods for studying it, its aetiology and its relation to anterograde memory impairment. 


\subsubsection{Discussion}

\subsubsection{Is remote memory impaired in temporal lobe epilepsy? If so, for which types of material and is there a laterality effect?}

Nine studies compared autobiographical episodic memory in TLE patients and healthy controls (Butler et al., 2007; Lah et al., 2004; Lah et al., 2006; Manes et al., 2005; Noulhiane et al., 2007; Upton et al., 1992; Viskontas et al., 2000; Voltzenlogel et al., 2007; Voltzenlogel et al., 2006). Of these, two found no evidence of impairment in the epilepsy group. The abstract published by Upton et al. (1992) reports no autobiographical memory impairment in a group of 29 TLE patients with subjective memory complaints. Lah et al. (2004) failed to find a deficit in the number of autobiographical events recalled by post-temporal lobectomy patients. This result is at odds with that reported in their subsequent study of pre-operative epilepsy patients (Lah et al., 2006) in which impairment was found. The remaining seven studies report significant impairment amongst TLE patients. One group (Voltzenlogel et al., 2006) found left TLE patients to be more impaired than right TLE patients on memory for autobiographic episodes, whereas two (Lah et al., 2006; Noulhiane et al., 2007) found greater impairment in the right TLE group.

Seven studies examined memory for personal semantics. Subtle differences were found in four (Barr et al., 1990; Butler et al., 2007; Lah et al., 2004; Lah et al., 2006). These were generally less marked than the differences in episodic memory. In the remaining three studies (Upton et al., 1992; Viskontas et al., 2000; Voltzenlogel et al., 2006), the failure to find a difference between patients and controls could be explained by a ceiling effect.

Five studies examined memory for public events (Barr et al., 1990; Bergin et al., 2000; Lah et al., 2004; Lah et al., 2006; Voltzenlogel et al., 2006). All found significant impairment amongst epilepsy patients compared with healthy controls. Deficits were also found for other non-personal, semantic memories including famous faces, famous names, television programmes and famous scenes. In four of these studies (Barr et al., 1990; Lah et al., 2004; Lah et al., 2006; Voltzenlogel et al., 2006), patients with left TLE were more severely impaired that those with right TLE, a result 
also described in the abstract by O'Connor et al. (1999), who did not include a healthy control group.

\subsubsection{Methodological issues in the assessment of remote memory}

The study of remote memory poses several methodological challenges:

i) The original acquisition of the memories in question is beyond the control of the investigator. Thus it is difficult to be certain whether apparently 'missing' memories were ever actually acquired or retained for any length of time. Several factors may be in play here. First, it is important to consider the possibility that a longstanding anterograde memory deficit might explain an individual's RMI, through defective initial acquisition or consolidation. In some patients, for example some of those with TEA, it is possible to be confident that remote memories had been successfully acquired as they were captured in family photographs that were reviewed regularly or had been repeatedly discussed. Second, even individuals with normal anterograde memory function differ markedly in their exposure to and interest in current events (Kapur et al., 1999). This is rarely taken into account in research studies. Third, the persistence of autobiographical event memories is closely linked to contextual factors such as emotional arousal ('flashbulb' memories), current goals (Conway and PleydellPearce, 2000) and life period (Rubin and Schulkind, 1997): these must be considered in interpreting variations in autobiographical recollection.

ii) The 'age' of a memory - often important in theorising about consolidation processes - cannot be straightforwardly equated with the time since its initial acquisition: re-exposure and rehearsal are important confounding factors.

iii) There is no universally accepted method for eliciting and probing remote memories. For autobiographical memories, cuing may be minimal, for example: "tell me about something that happened to you in your 30's"; moderate: "tell me about an incident involving a boat" (Crovitz, 1974); or highly specific and derived from information obtained from a close friend or relative. Cues may be verbal or non-verbal (e.g. photographs). 
Similarly, the third-party validation of reported memories can be difficult, although frank confabulation is thought to be rare (Tranel and Jones, 2006).

iv) There is a range of possible methods for scoring the subject's report ranging from simple true/false discrimination, to detailed textual analysis of transcribed responses (Levine et al., 2002). This and the previous consideration are reflected in the variety of tools available for the study of autobiographical memory, several of which are mentioned in the following paragraph.

Four studies used the Autobiographical Memory Interview (AMI) (Kopelman et al., 1989) to investigate episodic and semantic autobiographical memory (O’Connor et al., 1999; Upton et al., 1992; Viskontas et al., 2000; Voltzenlogel et al., 2006). Of these, none found a difference in personal semantic memory and just two found a deficit in episodic memory, amongst TLE patients. However, the AMI has been criticised as being relatively insensitive to milder or patchy retrograde memory deficiencies (Fujii et al., 2000; Manes et al., 2001). Two studies used an autobiographical fluency test, whereby subjects are given a fixed amount of time to generate as many names of acquaintances or personally experienced events as possible from a particular life period (Lah et al., 2004; Lah et al., 2006). Notably, this test does not examine detailed episodic memories, but awards points even for vague memories of recurrent events and depends heavily on intact executive control mechanisms. Finally, five studies used quite specific probes to prompt recall for events from different life periods and scored memories according to the degree of experiential richness conveyed by the subject (Butler et al., 2007; Manes et al., 2005; Noulhiane et al., 2007; Voltzenlogel et al., 2007; Voltzenlogel et al., 2006). These all found impairment amongst TLE patients.

Particular challenges arise when investigating remote memory in epilepsy. First, the clinical onset of seizures, especially in TLE, often dates back many years to childhood or adolescence, and the underlying pathological processes perhaps even to infancy. It can therefore be particularly difficult to distinguish between the anterograde and retrograde components of memory impairment. Second, the presence of an active seizure focus in a young brain induces remodelling of the neural pathways involved in 
memory so that the cognitive and neural processes occurring in patients and controls may differ. Third, a diagnosis of epilepsy often has further consequences that may influence the formation and retrieval of memories, including the use of medication, interrupted schooling and restricted employment opportunities. Fourth, the interpretation of the effects of the site and laterality of pathology upon remote memory is not straightforward: even when structural imaging reveals pathology only in one temporal lobe, there may be impairment of function remotely in both ipsilateral and contralateral hemispheres (Bernasconi et al., 2004; Diehl et al., 2003; Liu et al., 2003; Vermathen et al., 2002).

\subsubsection{The nature of the deficit: the relationship between anterograde and remote memory loss and the temporal gradient of remote memory}

RMI can, in principle, reflect a deficit at any stage of memory processing: acquisition, consolidation, storage or retrieval. Strong correlations between measures of anterograde and remote memory would raise the possibility that an impairment of remote memory is due to failure of acquisition. On the other hand, a lack of correlation between measures of anterograde and remote memory, or apparent loss of memories that were previously accessible, point to failure of storage or retrieval. However, as Kopelman (2000) has pointed out, it is important to remember that, in assessments of anterograde and remote memory impairment, "like" is not always being compared with "like". Tests of remote memory may, for example, require greater effort or be more dependent on visual imagery than tests of anterograde memory. Scoring methods for remote memories (e.g. that described by Levine et al. (2002)) may be more exacting than those usually applied in anterograde tests.

Whilst the majority of single cases reviewed here show profound retrograde loss of remote memories for events and facts learned decades before the onset of epilepsy but relatively preserved new learning, the evidence bearing on these matters in the group studies is mixed. Barr et al. (1990), examining post-surgical TLE patients, found significant correlations between two measures of remotely acquired semantic memory - famous face naming and recognition of television programs - and verbal anterograde memory, but only amongst left temporal lobectomy patients. Lah and colleagues, in studying both pre-surgical (Lah et al., 2006) and post-surgical (Lah et 
al., 2004) patients, found numerous correlations between remote memory measures and both anterograde memory and language tasks. Only autobiographical event memory failed to correlate with any anterograde memory task. In contrast, Butler et al. (2007) describe a group of 22 patients with TEA who performed normally on standard tests of anterograde memory but showed substantial impairment on a test of autobiographical memory across many decades. Finally, Voltzenlogel et al. (2007) found that patients who underwent right temporal lobectomy showed improvement in their ability to remember recent events after surgery, and that this change correlated with their improvement on delayed recall of a short story. Summarising these results, it appears that performance on standard anterograde memory tests can account for at least some of the impairment seen in remote memory amongst patients with TLE, both before and after surgery. Nevertheless, there remain cases, particularly in the TEA literature, where other factors must play a role. ALF may be part of the explanation, but this cannot be responsible for the loss of remote memories that had previously been accessible for many years, a phenomenon commonly reported by patients with TEA. Thus the impairment of remote memory that occurs in patients with epilepsy is likely to have a number of distinct causes, each with its own underlying pathophysiology.

Several studies have examined the distribution of RMI across the life span - its 'temporal gradient' (Barr et al., 1990; Butler et al., 2007; Lah et al., 2004; Lah et al., 2006; Manes et al., 2005; Noulhiane et al., 2007; Viskontas et al., 2000). Of the six studies that investigated autobiographical episodic memory, all except Lah et al. (2004) found a significant group effect: patients scored lower than controls across the lifetime. However, only Manes et al. (2005) found a group by time interaction and, here, the patients were better at recalling more recent memories: there was a reversed temporal gradient. In three studies (Butler et al., 2007; Manes et al., 2005; Viskontas et al., 2000), memory was shown to be impaired for events that occurred prior to the clinical onset of seizures. A notable feature of the RMI described by patients with TEA is that it appears to be patchy, with 'islands' of preserved memory (Butler et al., 2007; Manes et al., 2005; Zeman et al., 1998). This symptom remains to be explored using appropriate objective measures, but may provide important clues about the underlying mechanism. 
No study found a significant group by time interaction for personal semantic memory. Memory for public events from different life periods was investigated in three studies (Barr et al., 1990; Lah et al., 2004; Lah et al., 2006). The only significant group by time interaction was found for patients who had undergone a right temporal lobectomy, who were worse at recognising more recent public events (Lah et al., 2006). Since this difference was not found for pre-operative patients with right-sided TLE, the authors suggest that it may be a direct result of the surgery rather than the epilepsy itself.

\subsubsection{Aetiology and pathophysiology of RMI in epilepsy}

Six studies examined the possible causes of RMI in epilepsy (Bergin et al., 2000; Lah et al., 2004; Lah et al., 2006; Noulhiane et al., 2007; Viskontas et al., 2000; Voltzenlogel et al., 2006). Data were variously gathered on demographics, seizure variables (lifetime number of all or only generalised tonic-clonic seizures, age of onset, current seizure frequency, duration of epilepsy), structural variables (underlying pathology, extent of any temporal lobe excision) and anticonvulsant therapy.

\section{Seizures}

No study identified correlations between seizure-related variables and autobiographical memory performance. However, several factors were found to correlate negatively with remote memory for public events and general knowledge including: a greater lifetime number of generalised tonic-clonic seizures (Bergin et al., 2000), earlier age of epilepsy onset (Lah et al., 2006) and continuing post-operative seizures (Lah et al., 2004). However, contradictory findings are reported by other studies that found no effect of generalised tonic-clonic seizures (Lah et al., 2004; Lah et al., 2006), age of epilepsy onset (Lah et al., 2004; Noulhiane et al., 2007; Voltzenlogel et al., 2006), duration of epilepsy (Lah et al., 2004; Voltzenlogel et al., 2006) or seizure frequency (Voltzenlogel et al., 2006). There are several possible reasons for these inconsistencies. For example, studies often rely upon retrospective estimates of seizure frequency from the patient or their family, a method that may be inaccurate, especially when the frequency has varied a lot over time. Furthermore, 
studies rarely controlled for factors such as mood, general intelligence and language abilities, which are important determinants of test performance. Additional variation is introduced by the investigation of different patient populations and the use of different measures of memory.

\section{Structural lesions}

The lack of firm evidence for a correlation between seizure variables and remote memory performance led three groups (Bergin et al., 2000; Lah et al., 2004; Voltzenlogel et al., 2006) to conclude that temporal lobe pathology, rather than the resulting seizures, was responsible for patients' difficulty in remembering the past. Recently, Noulhiane et al. (2007), investigating a small group $(n=22)$ of postoperative TLE patients, found correlations between autobiographical memory scores and the volumes of residual medial temporal lobe structures, particularly in the right hemisphere, as measured on MRI scans. However, in other studies, remote semantic memory impairment was found not to vary with aetiology (hippocampal sclerosis or not) (Viskontas et al., 2000; Voltzenlogel et al., 2006), pre- or post-operative status (Viskontas et al., 2000) or the extent of operative excision (Lah et al., 2004). Furthermore, amongst patients with TEA (Butler et al., 2007; Manes et al., 2005; Manes et al., 2001) and in the five case reports discussed above, extensive retrograde amnesia occurs with minimal or no clinically apparent structural brain damage. Kapur (2000) has suggested that, in such cases, intermittent clinical or subclinical seizure activity beginning in the medial temporal lobes may disrupt neocortical networks that act as storage or retrieval sites for remote memory.

Functional brain imaging studies of activation patterns during autobiographical memory recall (see Maguire (2001) and Svoboda et al. (2006) for reviews) implicate the involvement of a wide network of brain regions, including frontal, temporal and posterior cortices, as well as the cerebellum and subcortical structures. The relative importance of these areas is likely to vary with factors such as the age of the memory, the emotional and perceptual content and the degree of personal significance (Svoboda et al., 2006). Future studies of patients with disproportionate impairments of autobiographical memory, such as those considered here, using both structural and 
functional imaging in life, together with post-mortem examination of brain tissue, will help to clarify which brain regions and processes contribute to this deficit.

\section{Anticonvulsant medication}

Two studies found a positive correlation between remote semantic memory loss and the number of anticonvulsants taken (Lah et al., 2004; Lah et al., 2006). Interpretation of this result is confounded by the close relationship between medication dose and the severity of epilepsy. It would be possible to investigate the relation between remote memory and anticonvulsants more directly by studying patients taking these drugs for conditions other than epilepsy. However, it seems unlikely that this is the principle cause of the problem since, for example, TEA patients complain of profound autobiographical memory loss prior to starting treatment and are usually maintained on low dose monotherapy (Butler et al., 2007).

\section{Psychosocial factors}

Mood disturbance is associated with 'overgeneral' memory for public and autobiographical events (Warren, 2007). It is therefore important to consider the possibility that mood disturbance associated with epilepsy may be playing a part in producing RMI. Only one of the studies reports data pertaining to this: Butler et al. (2007) found no difference in past or present diagnoses of major depression or generalised anxiety, and only a subtle difference in scores on the Hospital Anxiety and Depression Scale between patients and controls. In future explorations of the mechanisms underlying RMI, detailed data on possible mood disturbance will be essential.

\subsection{Summary}

In this chapter, two forms of memory impairment related to epilepsy have been reviewed, which together may help partially to explain the high prevalence of memory complaints in people with epilepsy who score normally on standard neuropsychological tests. There are several methodological challenges that arise in the investigation of ALF and RMI. A specific impairment in very long-term memory 
retention (i.e. ALF) is most clearly demonstrated when recollection at short delays is intact. However, some patients with epilepsy, particularly those with intractable TLE, show impairment on standard memory tests, making predictions about their expected performance at longer delays difficult. The investigation of remote memory has to assume that memories were acquired normally, an assumption that may not be justified in patients with chronic epilepsy.

From a theoretical standpoint, ALF and RMI are important. ALF suggests a deficit in a long-term memory consolidation process - so-called 'systems consolidation' (see Chapter 1) - which is thought, on the standard theory, to involve interaction between the hippocampus and neocortex and lead to eventual independence of at least certain types of memory trace upon the medial temporal lobes. Failure of this process might reflect subtle hippocampal damage - and hence be considered a mild version of the amnesic syndrome - or be due to intermittent functional impairment related to seizure discharges. Several recent studies have drawn attention to a possible role for sleep in memory consolidation (see for e.g. Walker and Stickgold (2006)). The frequent occurrence of amnesia on waking in TEA suggests one possible explanation for ALF in this condition - that subclinical nocturnal seizure discharges disrupt memory consolidation - a possibility that merits further study using a combination of polysomnography and memory measures.

RMI may be due to i) long-standing problems with memory encoding and consolidation, ii) impairment of memory retrieval processes or iii) a true loss of established memory traces. In TEA, the late-onset, extensive, consistent and sometimes isolated autobiographical memory loss suggests the third of these alternatives, although the mechanism by which established memories are disrupted remains unclear. Again, seizures or structural pathology may play a causal role.

Future investigations of long-term forgetting and remote memory in epilepsy will need to address important questions regarding their prevalence, causes and potential treatment whilst paying careful attention to the methodological issues raised in this chapter. Patients with TEA, whose seizures begin later in life and whose anterograde memory across short delays is often normal, form an ideal study population. In the following chapter, I report the results of an investigation into the structural correlates 
Chapter 5

of TEA and its associated memory deficits, in which structural MRI data were used to analyse regional brain volumes and their relation to performance on memory tests. 
Chapter 5 


\section{CHAPTER SIX}

\section{The radiological characteristics of transient epileptic amnesia}

\subsection{Introduction}

In the previous two chapters, I described the neuropsychological profile of patients with transient epileptic amnesia (TEA), particularly the phenomena of accelerated long-term forgetting (ALF) and autobiographical amnesia, and examined the evidence that these relatively novel forms of memory impairment can be identified in other forms of epilepsy. I now turn to the neuroimaging data collected from participants in the TIME Project in order to investigate the structural correlates of TEA and its associated memory deficits.

The aetiology of TEA is unknown. As discussed in Chapter 3, structural lesions thought to be responsible for the epilepsy were found in only a small proportion of cases reported in the literature. It has been previously suggested (Zeman et al., 1998) that the seizure focus in TEA may result from cerebrovascular disease. However, in Chapter 2 it was shown that risk factors for cerebrovascular disease are no more prevalent among patients with TEA than control subjects. Neuroimaging is a more direct marker of cerebrovascular disease. Besides revealing previous infarction and haemorrhagic stroke, magnetic resonance imaging (MRI) can disclose signs of covert small-vessel disease. White matter hyperintensities (WMH), seen on T2-weighted and fluid-attenuated inversion recovery (FLAIR) MRI sequences, represent regions of scattered white matter loss with increased tissue water content. They are common in older adults and correspond to ischaemia from hypoperfusion and small-vessel disease (Fazekas et al., 1993). Their extent correlates with certain cerebrovascular risk factors including hypertension (Dufouil et al., 2001) and diabetes (Schmidt et al., 1992). Moreover, their presence is associated with cognitive decline (Breteler et al., 1994; De Groot et al., 2000). Enlarged perivascular spaces (EPVS) are another ageassociated feature seen on MRI and appear as punctate or linear hyperintensities, isointense with cerebrospinal fluid on T2-weighted sequences. They are thought to correspond to extensions of the subarachnoid space surrounding the penetrating 
arteries (Braffman et al., 1988). EPVS have been shown to correlate with WMH load and with cognitive function (MacLullich et al., 2004). These imaging abnormalities may, therefore, provide a robust objective indication of the presence and extent of small-vessel disease.

MRI can also be used to quantify cortical atrophy associated with epilepsy and its related cognitive deficits. Most studies of memory in epilepsy have concentrated on medically refractory TLE, in which there is often significant structural damage to medial temporal lobe (MTL) regions including the hippocampus. Using MRI, such focal atrophy may be measured by calculating the volumes of manually traced regions of interest (Bernasconi et al., 2003; Cendes et al., 1993; Coste et al., 2002; Goncalves Pereira et al., 2005; Lencz et al., 1992) or by automated techniques such as voxelbased morphometry (VBM) (Ashburner and Friston, 2000; Bernasconi et al., 2004). These methods allow exploration of the relations between cognitive performance, clinical variables and structural brain pathology. Some studies of TLE have demonstrated associations between, for example, hippocampal volume and memory performance (Alessio et al., 2006; Kilpatrick et al., 1997; Reminger et al., 2004). However, the investigation of memory dysfunction in medically refractory TLE is complicated by its varied origins, associated cognitive deficits and by the severity of the clinical syndrome.

As will be evident from the preceding chapters, TEA is a form of epilepsy that has a particularly intimate relationship with memory. In TEA, the principle manifestation of seizures is recurrent episodes of isolated memory loss (Butler et al., 2007; Kapur, 1990; Zeman et al., 1998). Furthermore, even once attacks have ceased with anticonvulsant medication, many patients complain of persisting memory difficulties. Performance on standard neuropsychological tests is usually normal. However, patients show accelerated forgetting of new material over extended intervals and memory deficits for autobiographical events that occurred up to 40 years before the clinical onset of seizures (Butler et al., 2007; Manes et al., 2005; Manes et al., 2001). These unusual phenomena are of both clinical and theoretical significance. Their pathophysiological bases remain unknown. 
In this chapter, I first present the clinical neuroimaging findings from the TIME Project and an analysis of the radiological markers of microvascular disease. I then report a volumetric analysis of the structural MRI data using two distinct techniques manual delineation of specified regions of interest and voxel-based morphometry, an automated, observer-independent method that calculates grey matter volume at every voxel across the brain. Each technique has its advantages and limitations. Using both provides more robust results. In the volumetric analyses, two principle questions were addressed: i) whether TEA is associated with regional brain atrophy, particularly in the temporal lobes, and ii) whether any structural correlates of the interictal memory deficits can be identified.

\subsection{Methods}

\subsubsection{Subjects}

50 patients fulfilling the diagnostic criteria for TEA (Butler et al., 2007; Zeman et al., 1998) were recruited to the TIME Project and were assessed by a single investigator (CRB). A detailed clinical evaluation and neuropsychological assessment were performed (see Chapter 4 and summary below). MRI brain scanning was carried out according to the TIME protocol (see below and Appendix I) in 43 patients. A further 4 patients had previously undergone clinical MR imaging in other centres, the results of which were made available, but could not travel to Cambridge or Edinburgh for the research scan. Computerised tomography (CT) was carried out in two patients who had cardiac pacemakers and could therefore not undergo MRI. One patient did not consent to imaging. A subset of 22 scanned patients, in whom standard anterograde memory test performance was normal, underwent further testing of memory retention over an extended delay of three weeks.

Twenty age- and education-matched, neurologically normal control subjects also underwent MRI scanning, standard neuropsychological assessment, autobiographical memory testing and assessment of forgetting over a three-week interval. T2-weighted and FLAIR data from an additional 50 healthy male control subjects were made available from the Ageing Brain study, run by the University of Edinburgh Division of Clinical Neurosciences for the assessment of markers of microvascular disease. 


\subsubsection{Neuropsychological assessment}

The neuropsychological assessment was described in Chapter 4 and is summarised briefly below.

\section{General neuropsychology}

Standard tests were used to assess general intelligence (Wechsler Abbreviated Scale of Intelligence (Wechsler, 1999)), anterograde memory (immediate and 30 minute delayed recall of the Logical Memory story (from Wechsler Memory Scale-IIIR (Wechsler, 1997)), copy and 30 minute delayed recall of the Rey-Osterreith complex figure (Osterreith and Rey, 1944), the Recognition Memory Test for words and faces (Warrington, 1984)), language (Graded Naming Test (McKenna and Warrington, 1980)) and executive function (letter and category fluency, Wisconsin Card Sorting Test (WCST) (Kongs et al., 2000)). A summary measure of anterograde memory (zmem) was calculated for each patient by averaging the $\mathrm{z}$ scores for delayed Logical Memory recall, delayed Rey figure recall, word recognition and face recognition memory.

\section{Remote autobiographical memory}

A semi-structured interview, the Modified Autobiographical Memory Interview (MAMI) (see Appendix H), was used to assess memories for personal facts and personally experienced events from each decade of the subject's life (Butler et al., 2007). For two topics (e.g. 'holiday') from each decade, subjects were asked to answer five questions designed to probe semantic, or factual, memory (e.g. "How long did you stay?") and produce one detailed episodic memory (e.g. "Can you recall any incident, even if small, that occurred during your holiday in Seton Sands?"). Episodic memories were scored out of five according to their degree of specificity and experiential detail, based on the scheme described by (Graham and Hodges, 1997) (see Appendix H). A witness was present in all cases to corroborate the subject's account. For the purposes of this study, average scores across all decades were calculated for the semantic (MAMI-s) and the episodic (MAMI-e) components. In Chapter 4, only the MAMI results from subgroup 1 were presented in order to demonstrate autobiographical memory impairment even when standard anterograde 
memory performance is normal. In this chapter, I present MAMI scores for the whole patient group in order to increase the power of the volumetric analyses. However, similar results were obtained using just data from subgroup 1.

\section{Long-term forgetting}

This was tested in a subgroup of 22 patients (subgroup 1) and 20 control subjects. A list of 15 words (from the Rey Auditory Verbal Learning Task (Schmidt, 1996)) was orally presented over a minimum of five and a maximum of 15 trials until the subject attained $90 \%$ accuracy at free recall. Recall of the words was then tested at delays of 30 minutes, one week and three weeks. A similar procedure was used for recall of a short prose passage from the Rivermead Behavioural Memory Test (RBMT (Wilson et al., 1991)) and for reproduction of seven visually presented designs (from the Graham-Kendall Memory for Designs test (Graham and Kendall, 1968)). Long-term forgetting was defined as the percentage change in recall score between the 30 minute and the three week delay, a more negative score indicating greater forgetting. An average long-term forgetting measure (zALF) was calculated for each patient by averaging the long-term forgetting $\mathrm{z}$ scores across the three material types: word list, prose passage and designs.

The demographic, clinical and neuropsychological data are summarised in Table 6.1. The mean patient age was 67.7 years (standard deviation $(\mathrm{SD})=8.9$ ). There were 28 male and 13 female patients. The average IQ was well matched across groups (patient mean $=119.2(\mathrm{SD}=12.7)$, control mean $=121.2(\mathrm{SD}=14.9), \mathrm{p}=0.598)$. The patient group as a whole was impaired on standard memory tests relative to control subjects $(\mathrm{zmem}=-1.0 ; \mathrm{p}<0.001)$, and on the semantic and episodic components of the MAMI (MAMI-s: patient mean $=8.9(\mathrm{SD}=0.7)$, control mean $=9.5(\mathrm{SD}=0.4), \mathrm{p}<0.001$; MAMI-e: patient mean $=7.5(\mathrm{SD}=1.4)$, control mean $=9.1(\mathrm{SD}=0.7), \mathrm{p}<0.001)$. Patients scored higher on the HADS (patients: mean $=10.9(\mathrm{SD}=6.4)$; controls: mean $=7.5(\mathrm{SD}=3.1) ; \mathrm{p}=0.008)$. Subgroup 1 was unimpaired on all standard neuropsychological measures, but showed autobiographical memory impairment (MAMI-s: patient mean $=9.0(\mathrm{SD}=0.6), \mathrm{p}<0.001$; MAMI-e: patient mean $=7.7$ $(\mathrm{SD}=1.1), \mathrm{p}<0.001)$ and $\operatorname{ALF}(\mathrm{zALF}=-1.5(\mathrm{SD}=1.3) ; \mathrm{p}<0.001)$ 
Table 6.1 Demographic, clinical and neuropsychological characteristics of TEA patients and controls

\section{Age (years)}

Sex distribution

Education (years)

Age at onset of seizures (years)

Total number of seizures

Seizure frequency (per year)

Duration of epilepsy (months)

Full scale IQ

Logical memory delayed recall (25)

Rey figure delayed recall (36)

RMT (words) (50)

RMT (faces) (50)

zmem

Rey figure copy (36)

Graded faces (60)

Graded naming (30)

Letter fluency

Category fluency

WCST categories

HADS (42)

MAMI-s (10)

MAMI-e (10)

zALF
All patients $(n=41)$

mean (SD)

67.7 (8.9)

$28 \mathrm{M} / 13 \mathrm{~F}$

$12.5(3.0)$

$61.1(9.2)$

$18.5(17.7)$

$13.2(11.7)$

$81.3(69.4)$

$119.2(12.7)$

$11.9(5.1)^{*}$

$15.6(6.4)^{*}$

$46.3(3.6)^{*}$

$41.2(5.2)^{* *}$

$-1.0(1.1)^{* *}$

$34.6(3.0)$

$40.3(9.6)$

$21.7(5.0)$

$41.8(14.1)$

$19.9(5.9)$

$2.9(1.3)$

$10.9(6.4)^{*}$

$8.9(0.7)^{\star *}$

$7.5(1.4)^{\star *}$

$\mathrm{n} / \mathrm{a}$
Subgroup 1 ( $n=22)$

mean (SD)

$66.4(8.8)$

$12 \mathrm{M} / 10 \mathrm{~F}$

$13.2(3.0)$

$60.3(8.0)$

$20.5(19.3)$

$12.0(10.9)$

77.4 (71.9)

$124.7(10.7)$

$14.7(2.8)$

$16.7(5.6)$

47.7 (2.0)

43.4 (3.8)

$-0.4(0.6)$

$35.5(1.3)$

42.6 (8.1)

$23.4(3.0)$

$48.2(11.8)$

$22.5(5.2)$

$3.23(1.3)$

$10.4(6.1)$

$9.0(0.6)^{\star *}$

$7.7(1.1)^{* *}$

$-1.5(1.3)^{\star *}$
Controls $(n=20)$

mean (SD)

$67.5(8.6)$

$8 \mathrm{M} / 12 \mathrm{~F}$

13.0 (3.3)

$\mathrm{n} / \mathrm{a}$

$\mathrm{n} / \mathrm{a}$

$\mathrm{n} / \mathrm{a}$

$\mathrm{n} / \mathrm{a}$

$121.2(14.9)$

$14.6(3.7)$

$19.9(6.3)$

$48.4(1.7)$

$45.4(2.8)$

$0.0(0.7)$

$35.4(1.2)$

$44.6(7.4)$

$23.9(3.8)$

$45.0(10.3)$

$22.7(4.6)$

$3.8(1.3)$

$7.5(3.1)$

$9.5(0.4)$

$9.1(0.7)$

$0.0(1.0)$

* significantly different from control group $(p<0.05)$

** significantly different from control group $(p<0.001)$

$\mathrm{ALF}=$ accelerated long-term forgetting; $\mathrm{HADS}=$ Hospital Anxiety and Depression Scale; $I Q=$ intelligence quotient; $\mathrm{MAMI}=$ Modified Autobiographical Memory Interview; MAMI-s = MAMI semantic memory subscore; MAMI-e = MAMl episodic

memory subscore; $\mathrm{n} / \mathrm{a}=$ not applicable; RMT = Recognition Memory Test; $\mathrm{WCST}=$ Wisconsin Card Sorting Test; $\mathrm{zALF}=$

ALF summary score; zmem = summary anterograde memory score.

\subsubsection{Magnetic resonance imaging}

Magnetic resonance imaging was performed on a 1.5T GE Signa LX scanner (GE Medical Systems, Milwaukee, USA) at the SFC Brain Imaging Centre for Scotland, Western General Hospital, Edinburgh or on a scanner of the same model at the MRIS Unit, Addenbrooke's Hospital, Cambridge. The MR sequences used were T1weighted, T2-weighted, fluid attenuated inversion recovery (FLAIR), gradient echo and diffusion-weighted imaging (see Appendix I). 3D volumetric data were acquired in the coronal plane, perpendicular to an axis connecting the anterior and posterior commissures (AC-PC axis), using a coronal T1-weighted three-dimensional spoiled gradient-echo sequence (echo time $=3.36 \mathrm{msec}$, repetition time $=8.20 \mathrm{msec}$, flip angle $=15^{\circ}$, slice thickness $=1.7 \mathrm{~mm}$, slice gap $=0 \mathrm{~mm}$, field of view $=22 \mathrm{~cm}$, 
matrix $=256 \times 192)$. All scans were reviewed for clinical abnormalities by an experienced neuroradiologist ${ }^{1}$.

\subsubsection{Neuroimaging markers of cerebrovascular disease}

Axial T2-weighted and FLAIR images were rated for WMH using the Fazekas scale (Fazekas et al., 1987) ${ }^{1}$. This widely used scale distinguishes between periventricular and deep white matter hyperintensities. Scores are allocated according to the criteria described and illustrated in Figure 6.1 (a). EPVS were quantified in the centrum semiovale, basal ganglia and hippocampus using the method of MacLullich et al (2004). The rating criteria are described and illustrated in Figure 6.1 (b).

\subsubsection{Manual volumetric analysis}

Volumetric analysis was performed using Analyze 7.0 software (Mayo Clinic, Rochester, MN, USA), which allows simultaneous viewing in coronal, sagittal and horizontal orientations. The data were resampled onto a $0.86 \mathrm{~mm}$ voxel grid and alignment to the AC-PC axis was checked manually. The hippocampus and amygdala were segmented manually using the protocol described by Pruessner et al. (2000). Further segmentation of the hippocampus into head, body and tail sections was performed according to the landmarks used by Malykhin et al. (2007) (see Figure 6.2). Volumes were corrected for total intracranial volume (TIV), estimated using the method described by Eritaia et al. (2000). The correction was performed using the formula: adjusted volume of structure $x=($ volume of structure $x /$ TIV) $*$ mean TIV of all participants. A single investigator ${ }^{2}$, blinded to the participants' details, performed the segmentation of all images. In order to assess intrarater reliability, 20 hemispheres were segmented twice, at an interval of one month. The intraclass reliability coefficients were 0.92 for hippocampal and 0.87 for amygdalar volumes, indicating a high degree of consistency in the delineation process.

\footnotetext{
${ }^{1}$ Professor Joanna Wardlaw, Consultant Neuroradiologist at the Department of Clinical Neurosciences, Western General Hospital, Edinburgh, reviewed the MRI scans obtained in the TIME Project for clinical abnormalities and performed the rating for WMH and EPVS on patient and control scans.

${ }^{2}$ Manual tracing of hippocampal and amygdalar volumes was carried out under my supervision by Amit Bhaduri, a fourth-year medical student, for his Special Study Component (SSC4).
} 


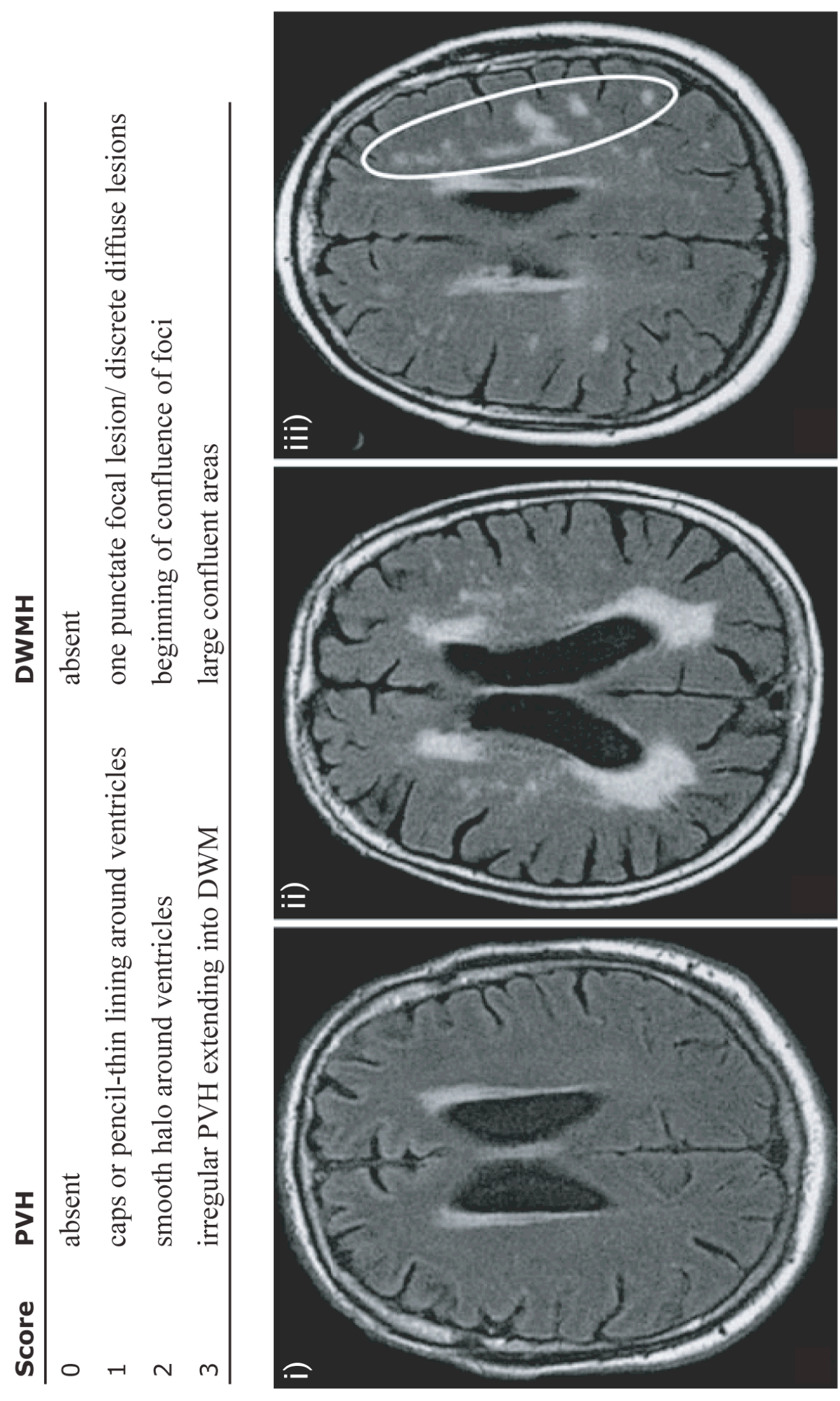

త్ర 


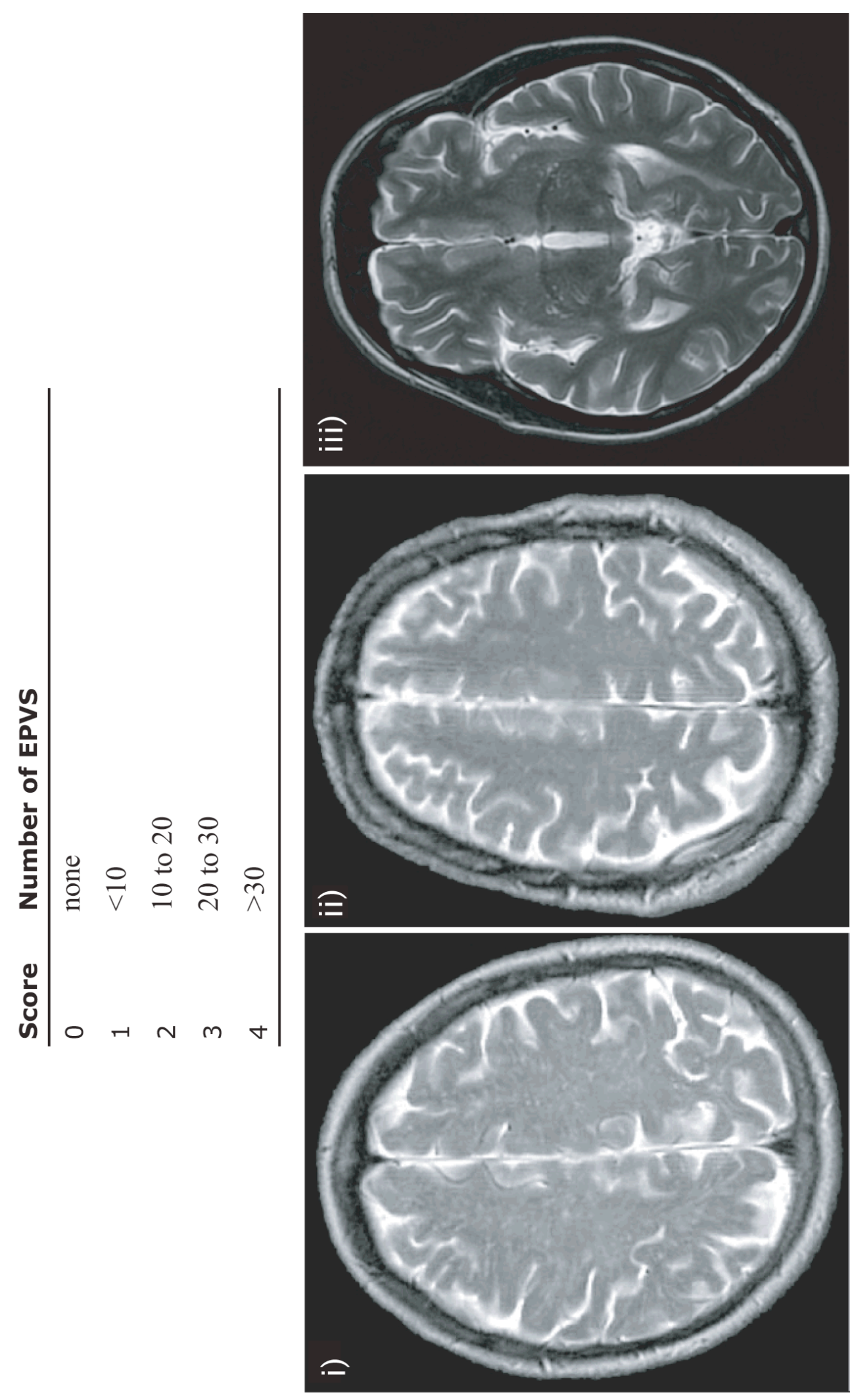

$\widehat{a}$ 
Figure 6.1 (above): (a) Scoring scheme and illustrative FLAIR MRI scans for white matter hyperintensities. Periventricular hyperintensities (PVH) and deep white matter hyperintensities (DWMH) are each scored out of 3 . The scans illustrate i) pencil thin lining around ventricles, ii) irregular PVH extending into deep white matter (DWM) and iii) the beginning of confluence of DWMH foci.

(b) Scoring scheme and illustrative T2-weighted MRI scans for enlarged perivascular spaces (EPVS). EPVS were rated in the centrum semiovale (CSO), basal ganglia and hippocampus. The scans illustrate i) $>30$ EPVS in the CSO, ii) $<10$ EPVS in the CSO and iii) 20 to 30 EPVS in the basal ganglia.

\subsubsection{Statistical analysis}

For the analysis of vascular changes on MRI, the datasets were dichotomised into mild (WMH score 0 or 1 , EPVS score 0 or 1 ) or moderate to severe (WMH score 2 or 3, EPVS score 2, 3 or 4). The relative proportions of scans rated as mild or as moderate/severe in the patient and control groups were compared using chi-squared tests. Neuropsychological and volumetric MRI data from the patient and control groups were compared using independent sample $t$ tests or the Mann-Whitney $U$ test where appropriate. Pearson's correlations were used to examine the relations between manual volumetric measurements, clinical variables and cognitive performance. The significance level for all analyses was set at $\mathrm{p}<0.05$. Statistical tests were performed using SPSS for Windows 15.0. 


\section{Chapter 6}

(a)

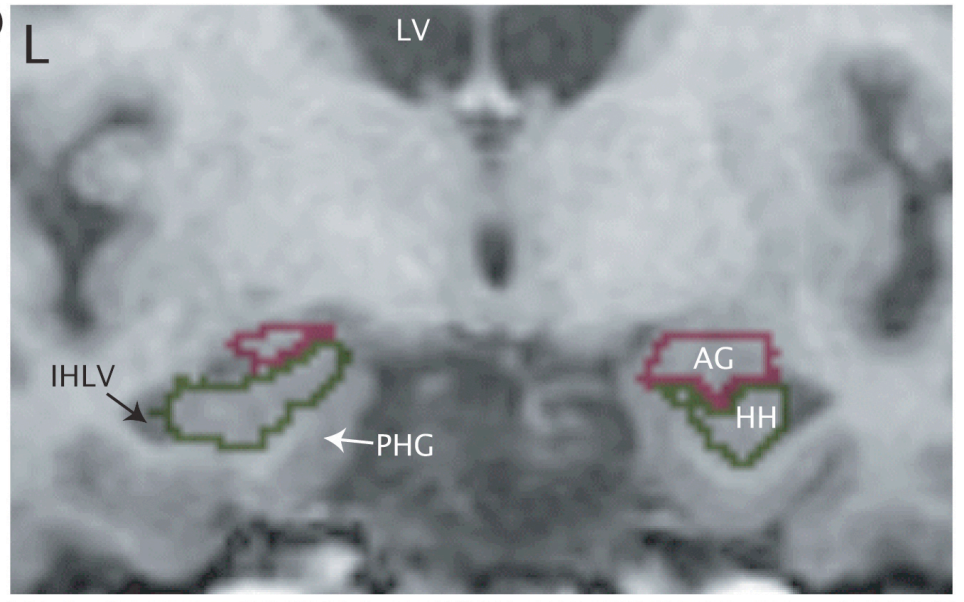

(b)

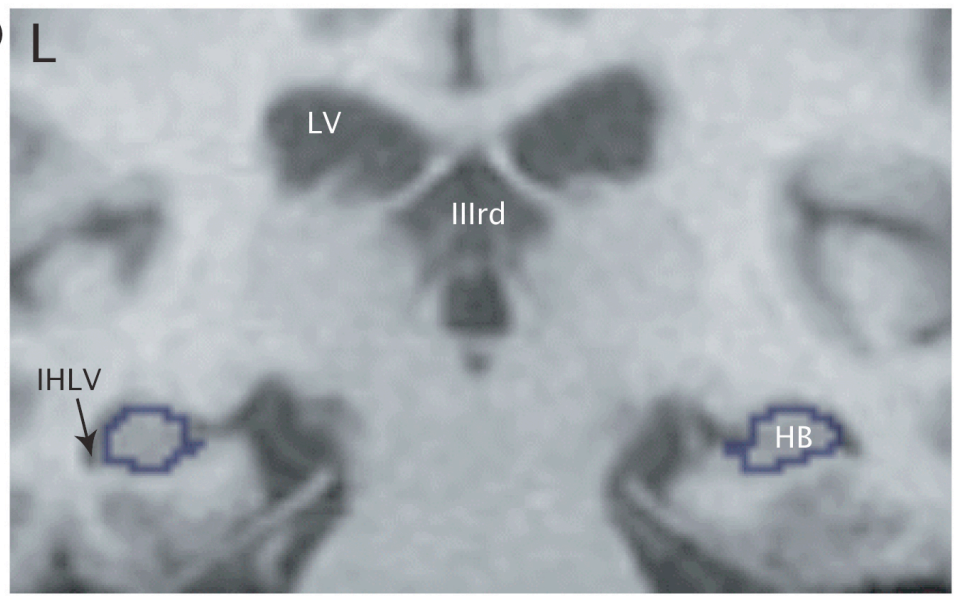

(c)

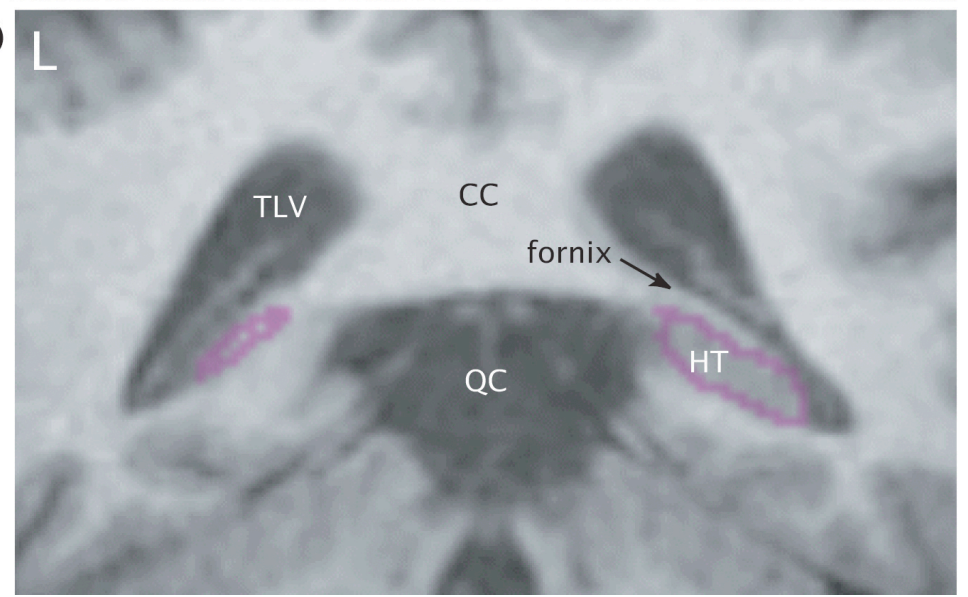

(d)
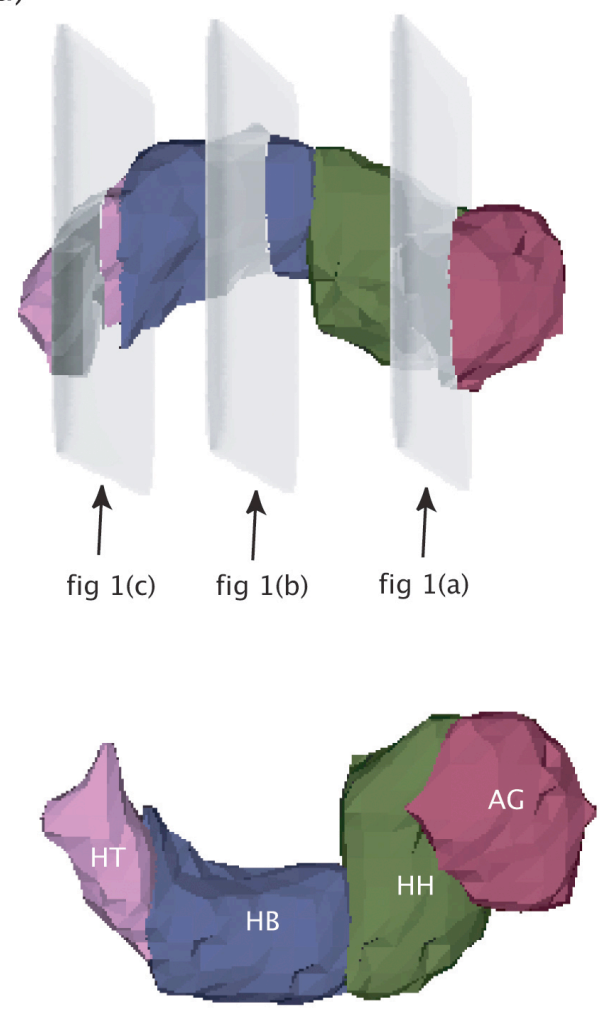

Figure 6.2: Coronal MRI scans (in native space) from a single subject showing the (a) amygdala and hippocampal head, (b) hippocampal body and (c) hippocampal tail. A three dimensional rendering of the volumes of interest $(\mathrm{d})$ indicates the planes of the coronal slices. $\mathrm{AG}=$ amygdala, $\mathrm{CC}=$ corpus callosum, $\mathrm{HH}=$ hippocampal head, $\mathrm{HB}=$ hippocampal body, HT = hippocampal tail, $\mathrm{L}=1 \mathrm{eft}, \mathrm{LV}=$ lateral ventricle, IHLV = inferior horn of the lateral ventricle, $\mathrm{PHG}=$ parahippocampal gyrus, TLV $=$ trigone of the lateral ventricle, $\mathrm{QC}=$ quadrigeminal cistern, $\mathrm{IIIrd}=$ third ventricle. 


\subsubsection{Voxel-based morphometry (VBM)}

VBM is a technique for voxel-wise analysis of local differences in brain tissue content (Ashburner and Friston, 2000) which has been used to study several types of brain disorder including temporal lobe epilepsy (Bonilha et al., 2007a; Bonilha et al., 2004; Keller et al., 2002a). The technique uses a spatial preprocessing stage followed by statistical analysis.

\section{Preprocessing $^{3}$}

The images were preprocessed using the following automated pipeline (AcostaCabronero et al., 2008): first, skull-stripping was performed using the hybrid watershed algorithm or HWA (Ségonne et al., 2004) in FreeSurfer v.3.04 (http://surfer.nmr.mgh.harvard.edu), which integrates an atlas-based term constraining the shape of the brain; stripped volumes were then bias-corrected using the nonparametric non-uniform intensity normalisation or N3 v.1.10 (Sled et al., 1998) with default arguments; and finally, a fine brain extraction that excludes venous sinuses and cerebrospinal fluid (CSF) was performed using the brain extraction tool v.2.1 or BET2 (Smith, 2002) in FSL v.3.3 (http://www.fmrib.ox.ac.uk/fsl) with fixed arguments: fractional intensity threshold, $\mathrm{f}$, set to 0.3 and vertical gradient, $\mathrm{g}$, set to 0 . All volumes were then spatially normalised and segmented using the unified segmentation model provided in SPM5 (Wellcome Department of Cognitive Neurology, London; http://www.fil.ion.ucl.ac.uk/spm), running on Matlab version 6.5.1 (MathWorks, Natick, MA). Previous investigations, however, suggested that SPM5 is more accurate if it does not attempt to estimate bias fields when nonuniformities are not present (Ashburner and Friston, 2005). Therefore, since radio frequency bias was corrected by N3 during the pre-processing step, non-uniformity correction was disabled from the SPM5 model by providing parameter settings that caused a negligible effect over the volume of interest; that is, bias regularisation was set to 10 and bias FWHM was set to $150-\mathrm{mm}$ cut-off. The segments were also modulated to compensate for volumetric differences introduced into the warped images. Modulation has the effect of preserving the total amount of grey matter

\footnotetext{
${ }^{3}$ This preprocessing pipeline was kindly suggested and much of it implemented by Julio AcostaCabronero and Peter Nestor from the Wolfson Brain Imaging Centre at the University of Cambridge.
} 
represented by multiplying by the relative volumes. Finally, grey matter segments were smoothed using an 8-mm FWHM isotropic Gaussian kernel. Spatial smoothing is required prior to statistical analysis in order to cope not only with systematic miswarping to template but also with inter-subject variations in anatomy, to improve the signal-to-noise ratio and to render the data more normally distributed.

\section{Statistical analysis}

The preprocessed grey matter images were analysed using SPM5. First, a group analysis was performed to compare regional differences in grey matter volume between patients and control subjects. Four covariates of no interest were entered into the statistical model: study centre, sex, age and TIV (calculated in SPM5 using a previously described method (Pengas et al., 2008)). Second, multiple-regression analyses were performed to identify brain regions where, in the patient population, grey matter volume varied linearly with a series of clinical and neuropsychological variables. The dependent variables investigated were: duration of epilepsy; lifetime number of seizures; performance on standard tests of anterograde memory in which the group as a whole was impaired relative to controls (delayed recall of the Logical Memory story and Rey complex figure; recognition memory for words and faces; and zmem); autobiographical memory (MAMI-s and MAMI-e) and, for the subgroup of patients in which long-term forgetting tests were administered, accelerated long-term forgetting (zALF). Again, study centre, sex, age and TIV were entered into the design matrix as covariates of no interest. A grey matter mask, created by binarising the probabilistic grey matter map, and a relative threshold mask of 0.8 , were applied. Resultant $t$ statistic maps were thresholded i) at $\mathrm{p}<0.001$ uncorrected for multiple comparisons in the temporal lobes, the presumed site of pathology in TEA, or ii) at $\mathrm{p}<0.05$ with false discovery rate (FDR) correction where there was no prior hypothesis.

\subsection{Results}

\subsubsection{Clinical neuroradiological assessment}

Clinical examination of the 47 MRI scans from patients with TEA revealed focal lesions potentially related to the epilepsy in 2 cases: in one (case 40) a temporal ridge 
meningioma and in the other (case 3) focal bilateral temporal lobe atrophy. The clinical characteristics of these two patients were indistinguishable from the rest of the group. Two patients had established but clinically silent infarcts, one in the territory of the right middle cerebral artery and the other in the right cerebellar hemisphere. One control subject had an established infarct in the left occipital cortex. None of these three subjects was scanned under the TIME protocol and hence none was included in the volumetric analyses.

\subsubsection{Neuroimaging markers of cerebrovascular disease}

The WMH and EPVS data are shown in Table 6.2. Patients and controls were well matched for age (patient mean $=68.2(\mathrm{SD}=8.8)$, control mean $=68.5(\mathrm{SD}=5.1), \mathrm{p}=$ 0.859 ) and sex (patients: 31 males; controls: 48 males; $\chi^{2}=0.001 ; p=0.976$ ). For comparison of proportions, the datasets were dichotomised into mild (WMH score 0 or 1, EPVS score 0 or 1) or moderate to severe (WMH score 2 or 3, EPVS score 2, 3 or 4). WMH scores were not significantly different between the patient and control group. The proportions of EPVS were different in the centrum semiovale $\left(\chi^{2}=5.340\right.$, $\mathrm{p}=0.021)$ and basal ganglia $\left(\chi^{2}=3.903, \mathrm{p}=0.048\right)$. Inspection of the raw data in Table 6.2 reveals that the overall pattern of results, with most subjects scoring 1 or 2 , was the same for both these regions, but a small number of patients scored slightly higher than expected, falling into the moderate/severe rather than the mild category.

\subsubsection{Manual volumetrics}

43 patients with TEA and 20 matched healthy control subjects underwent MR scanning according to the TIME Project protocol. As mentioned in section 6.3.1, two of these patients had focal structural lesions on clinical examination of the images and were consequently excluded from volumetric analyses. The results reported in the rest of this chapter pertain to the remaining 41 patients, all of whom were on anticonvulsant monotherapy and had been seizure-free for at least six months at the time of neuropsychological testing. 


\begin{tabular}{|c|c|c|c|}
\hline & Score & Patients $(n=47)$ & Controls $(n=70)$ \\
\hline Age & & $68.2(8.8)$ & $68.5(5.1)$ \\
\hline $\operatorname{Sex}(M / F)$ & & $31 / 16$ & $48 / 22$ \\
\hline \multicolumn{4}{|c|}{ White matter hyperintensities } \\
\hline \multirow[t]{4}{*}{$\mathrm{PVH}$} & 0 & $5(10 \%)$ & $2(3 \%)$ \\
\hline & 1 & $22(46 \%)$ & $31(44 \%)$ \\
\hline & 2 & $15(32 \%)$ & $33(47 \%)$ \\
\hline & 3 & $5(12 \%)$ & $4(6 \%)$ \\
\hline \multirow[t]{4}{*}{ DMWH } & 0 & $7(15 \%)$ & $4(6 \%)$ \\
\hline & 1 & $36(76 \%)$ & $62(89 \%)$ \\
\hline & 2 & $1(2 \%)$ & $4(6 \%)$ \\
\hline & 3 & $3(7 \%)$ & $0(0 \%)$ \\
\hline \multicolumn{4}{|c|}{ Enlarged perivascular spaces } \\
\hline \multirow[t]{5}{*}{ Centrum semiovale* } & 0 & $0(0 \%)$ & $1(1 \%)$ \\
\hline & 1 & $22(46 \%)$ & $47(67 \%)$ \\
\hline & 2 & $15(32 \%)$ & $15(21 \%)$ \\
\hline & 3 & $8(17 \%)$ & $7(10 \%)$ \\
\hline & 4 & $2(5 \%)$ & $0(0 \%)$ \\
\hline \multirow[t]{5}{*}{ Basal ganglia* } & 0 & $1(2 \%)$ & $0(0 \%)$ \\
\hline & 1 & $25(54 \%)$ & $52(74 \%)$ \\
\hline & 2 & $10(22 \%)$ & $12(17 \%)$ \\
\hline & 3 & $5(10 \%)$ & $5(7 \%)$ \\
\hline & 4 & $6(12 \%)$ & $1(1 \%)$ \\
\hline \multirow[t]{5}{*}{ Hippocampus } & 0 & $7(15 \%)$ & $1(1 \%)$ \\
\hline & 1 & $31(66 \%)$ & $48(69 \%)$ \\
\hline & 2 & $8(17 \%)$ & $20(29 \%)$ \\
\hline & 3 & $0(0 \%)$ & $1(1 \%)$ \\
\hline & 4 & $1(2 \%)$ & $0(0 \%)$ \\
\hline
\end{tabular}

* $p<0.05$ for comparison of proportions between patients and controls when data dichotomised into mild or moderate/severe (see text).

$\mathrm{PVH}=$ periventricular hyperintensities, $\mathrm{DWMH}=$ deep white matter hyperintensities

\section{Group analysis}

As shown in Table 6.3 and Figure 6.3, significant differences were found between patients and controls in the volumes, corrected for total intracranial volume, of both the left (patients: mean $=2776 \mathrm{~mm}^{3}(\mathrm{SD}=447)$; controls: mean $=3009 \mathrm{~mm}^{3}(\mathrm{SD}=$ $274) ; \mathrm{p}=0.016)$ and right (patients: mean $=2934 \mathrm{~mm}^{3}(\mathrm{SD}=498)$; controls: mean $=$ $\left.3201 \mathrm{~mm}^{3}(\mathrm{SD}=268) ; \mathrm{p}=0.009\right)$ hippocampus. When the longitudinal subdivisions 
of the hippocampus were compared, the hippocampal body volumes of patients and controls differed significantly in both the left (patients: mean $=920 \mathrm{~mm}^{3}(\mathrm{SD}=190)$; controls: mean $\left.=1032 \mathrm{~mm}^{3}(\mathrm{SD}=114) ; \mathrm{p}=0.006\right)$ and right (patients: mean $=968$ $\mathrm{mm}^{3}\left(\mathrm{SD}=163\right.$; controls: mean $\left.=1082 \mathrm{~mm}^{3}(\mathrm{SD}=138) ; \mathrm{p}=0.012\right)$ hemispheres. The volumes of the hippocampal head and tail did not differ significantly between groups in either hemisphere.

There was no significant difference detected in the volume of the left (patients: mean $=1281 \mathrm{~mm}^{3}(\mathrm{SD}=371)$; controls: mean $\left.=1271 \mathrm{~mm}^{3}(\mathrm{SD}=217) ; \mathrm{p}=0.898\right)$ or right (patients: mean $=1275 \mathrm{~mm}^{3}(\mathrm{SD}=304)$; controls: mean $=1265 \mathrm{~mm}^{3}(\mathrm{SD}=232) ; \mathrm{p}=$ 0.886 ) amygdala.

Table 6.3 Manually traced volumes of hippocampus (head, body and tail) and amygdala in TEA patients and controls corrected for total intracranial volume.

\begin{tabular}{llccc} 
& & \multicolumn{2}{c}{ Mean (SD) volume in $\mathrm{mm}^{3}$} & $p$ \\
Left hippocampus & total & $2776(447)$ & $3009(274)$ & 0.016 \\
& head & $1539(350)$ & $1636(228)$ & 0.275 \\
& body & $920(190)$ & $1032(114)$ & 0.006 \\
& tail & $319(98)$ & $342(119)$ & 0.432 \\
Right hippocampus & total & $2934(498)$ & $3201(268)$ & 0.009 \\
& head & $1672(383)$ & $1789(259)$ & 0.232 \\
& body & $968(163)$ & $1082(138)$ & 0.012 \\
& tail & $327(104)$ & $329(79)$ & 0.911 \\
Left amygdala & & $1281(371)$ & $1271(217)$ & 0.898 \\
Right amygdala & & $1275(304)$ & $1265(232)$ & 0.886
\end{tabular}




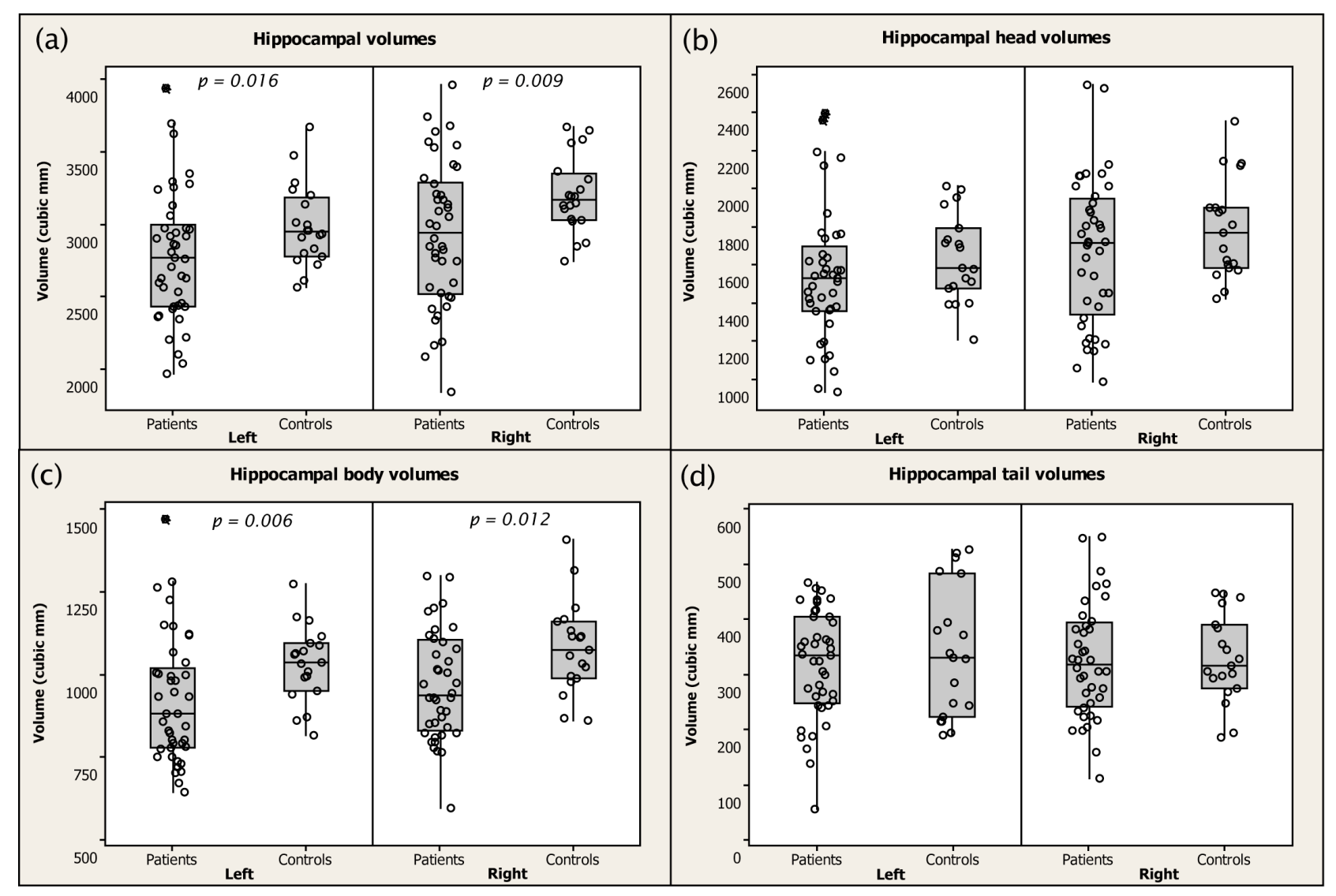

Figure 6.3: Boxplots showing medians (horizontal lines), interquartile ranges (grey boxes) and ranges (vertical lines) of volumes (adjusted for total intracranial volume) of the (a) hippocampus, (b) hippocampal head, (c) hippocampal body and (d) hippocampal tail, in patients and controls. Outliers are shown as crossed symbols. Where significant, $\mathrm{p}$ values are shown above the corresponding boxplot.

\section{Correlational analyses}

In order to identify potential causal factors for the interictal memory impairment seen in TEA patients, correlational analyses were carried out between hippocampal volumes, seizure variables and memory test scores. The results are shown in Table 6.4. Hippocampal volumes did not correlate with any neuropsychological measure in the control group. 


\begin{tabular}{|c|c|c|}
\hline & $\begin{array}{c}\text { LHC } \\
r\end{array}$ & $\underset{r}{R H C}$ \\
\hline age at onset & -0.037 & -0.004 \\
\hline duration of epilepsy & -0.170 & -0.260 \\
\hline seizure frequency & -0.025 & 0.005 \\
\hline number of seizures & $0.344^{*}$ & 0.286 \\
\hline zmem & 0.299 & $0.352^{*}$ \\
\hline Rey figure delayed recall & 0.180 & $0.315^{*}$ \\
\hline RMT (faces) & 0.294 & $0.320^{*}$ \\
\hline LM delayed recall & 0.073 & 0.158 \\
\hline RMT (words) & 0.266 & 0.228 \\
\hline MAMI-s & 0.082 & 0.131 \\
\hline MAMI-e & -0.165 & 0.084 \\
\hline zALF & -0.178 & 0.036 \\
\hline \multicolumn{3}{|c|}{$\begin{array}{l}\text { * } p<0.05 \\
\text { LHC = left hippocampus; } L M=\text { logical memory; RHC = right hippocampus; } r \\
=\text { Pearson's correlation coefficient; MAMI-s = MAMI semantic memory } \\
\text { subscore; MAMI-e = MAMI episodic memory subscore; RMT = Recognition } \\
\text { Memory Test; } z A L F=\text { ALF summary score; } \text { zmem = summary anterograde } \\
\text { memory score. }\end{array}$} \\
\hline
\end{tabular}

Seizure variables: In the patient group, there was no significant correlation between hippocampal volumes and duration of epilepsy or seizure frequency. A positive correlation was found between left hippocampal volume and the lifetime number of seizures $(\mathrm{r}=0.344, \mathrm{p}=0.028)$.

Standard anterograde memory: The overall anterograde memory score (zmem) showed a significant positive correlation with right $(r=0.352, p=0.024)$, and a trend towards correlation with left $(r=0.299, \mathrm{p}=0.057)$ hippocampal volume. Delayed recall of the Rey figure correlated with right hippocampal volume $(r=0.315 ; \mathrm{p}=$ 0.045). Recognition memory for faces correlated with right hippocampal volume $(r=$ 
$0.320, \mathrm{p}=0.041)$ and tended towards correlation with left hippocampal volume $(\mathrm{r}=$ $0.294, p=0.062$ ). Neither recognition memory for words nor logical memory scores correlated significantly with hippocampal volumes. In subgroup 1, the overall anterograde memory score (zmem) again correlated with right hippocampal volume (r $=0.457, \mathrm{p}=0.032)$ but not left hippocampal volume $(\mathrm{r}=0.367, \mathrm{p}=0.093)$.

Autobiographical memory: The average semantic (MAMI-s) and episodic (MAMI-e) autobiographical memory scores did not correlate with volumes of either hippocampus or with any seizure variable.

$A L F$ : The average score for forgetting over three weeks (zALF) was unrelated to hippocampal volume. No significant relationships were found between ALF scores and seizure variables.

\subsubsection{Voxel-based morphometry}

The results of the VBM analyses are shown in Figure 6.4 and Table 6.5. Results are reported at an uncorrected threshold of $\mathrm{p}<0.001$ within the temporal lobes, and at $\mathrm{p}<$ 0.05 FDR corrected in other brain regions. However, even at the uncorrected threshold, extratemporal clusters were rare and, where present, small ( $<40$ voxels).

Group analysis: No regions were identified in which patients had reduced grey matter volume. On the contrary, patients appeared to have increased grey matter in inferior temporal and parahippocampal regions bilaterally.

Epilepsy variables: No clusters were identified that correlated with duration of epilepsy, seizure frequency or total number of seizures.

Standard anterograde memory: In the patient group, the summary score of standard anterograde memory measures (zmem) correlated with a cluster of voxels in the left MTL. Recognition memory for faces correlated with bilateral medial temporal regions. Delayed recall of the Rey figure correlated with a cluster in the right hippocampus. 


\section{Chapter 6}

Autobiographical memory: In the patient group, neither episodic nor semantic MAMI scores correlated with any MTL voxels.

$A L F$ : In subgroup 1 , no brain regions were found that correlated with the summary score (zALF) or any of the individual tests of long-term forgetting.
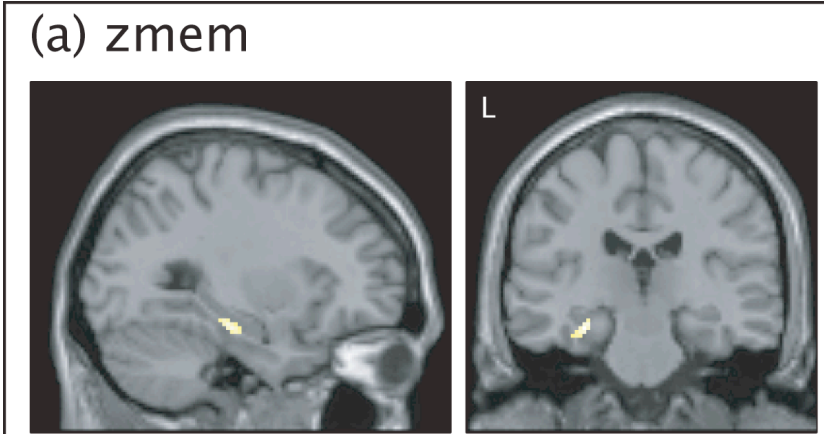

(b) RMT (faces)
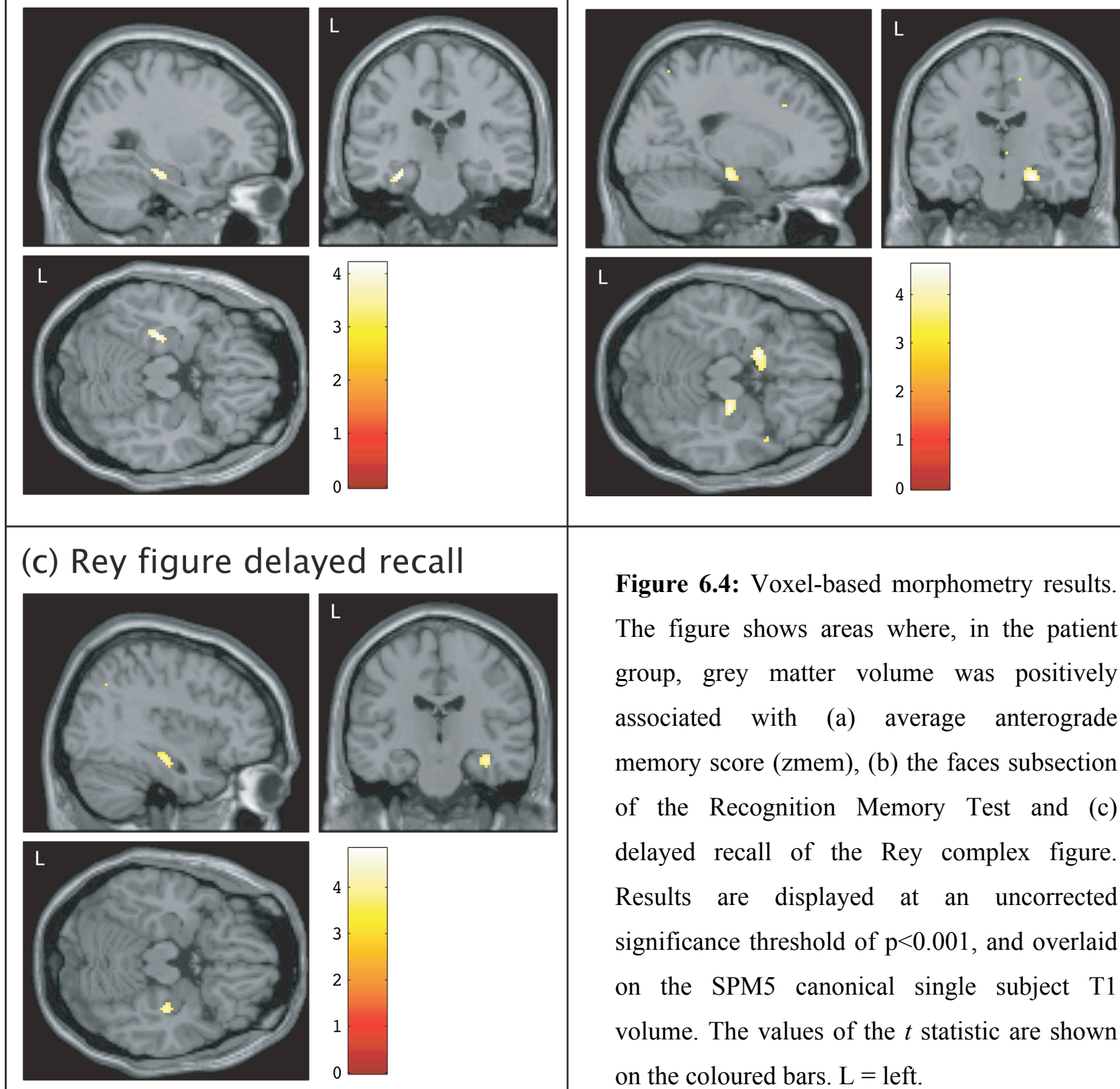

Figure 6.4: Voxel-based morphometry results. The figure shows areas where, in the patient group, grey matter volume was positively associated with (a) average anterograde memory score (zmem), (b) the faces subsection of the Recognition Memory Test and (c) delayed recall of the Rey complex figure. Results are displayed at an uncorrected significance threshold of $\mathrm{p}<0.001$, and overlaid on the SPM5 canonical single subject T1 volume. The values of the $t$ statistic are shown on the coloured bars. $\mathrm{L}=$ left. 
Table 6.5 Group and correlational VBM analyses. Results are reported at $p<0.001$ (uncorrected) for temporal lobe peaks and $p<0.05$ (FDR corrected) for extratemporal peaks.

$\begin{array}{lllllll}B A & x & y & \begin{array}{c}\text { cluster } \\ \text { size }\end{array} & p(F D R) & t & z\end{array}$

\section{controls $<$ patients}

$L$ inferior temporal gyrus

$37 \quad-38 \quad-42 \quad-14$

139

0.055

5.22

4.67

L parahippocampal gyrus

$20 \quad-42 \quad-28 \quad-20$

$0.055 \quad 4.86$

4.41

$R$ fusiform gyrus

37

$-22$

$-40$

0.055

4.74

4.31

$R$ inferior temporal gyrus

$\begin{array}{llll}37 & 38 & -40 & -16\end{array}$

109

0.074

$4.32 \quad 3.99$

$\mathrm{R}$ inferior temporal gyrus

20

$-12-24$

13

0.098

4.00

3.73

$\mathrm{R}$ parahippocampal gyrus

$36 \quad 36$

$0 \quad-36$

0.103

3.96

3.69

$30 \quad 26$

$-22 \quad-26$

17

$0.119 \quad 3.87$

3.62

zmem

L parahippocampal gyrus

$20-30$

$-24$

$-18$

59

0.968

4.19

3.75

RMT (faces)

R parahippocampal gyrus

$\begin{array}{lccccccc}20 & 24 & -16 & -18 & 73 & 0.265 & 4.61 & 4.05 \\ 34 & -18 & 4 & -18 & 85 & 0.265 & 4.59 & 4.04\end{array}$

L parahippocampal gyrus

$34-18$

$4 \quad-18$

85

0.265

4.04

Rey figure delayed recall

$\mathrm{R}$ hippocampus

$20 \quad 34$

$-20$

$-16$

51

0.383

4.23

3.78

$\mathrm{BA}=$ Brodmann area; FDR = false discovery rate corrected; RMT = Recognition Memory Test; $z$ mem = summary score for anterograde memory

\subsection{Discussion}

The work reported in this chapter was an investigation of the neuroradiological characteristics of TEA - in particular i) the presence of clinically apparent structural lesions potentially causing the seizures, ii) radiological signs of microvascular disease (WMH and EPVS) and iii) regional brain atrophy and its relationship with interictal memory scores. Clear structural lesions occur in a small minority of cases and, in this study, were located in the temporal lobes. Radiological markers of small-vessel disease were not clearly overrepresented in the patient group. TEA is, however, associated with subtle MTL atrophy, which correlates with performance on standard tests of anterograde memory, but not with ALF or with autobiographical amnesia. 
It has previously been hypothesised that the seizure focus in TEA may be due to cerebrovascular disease (Zeman et al., 1998). In Chapter 2, it was shown that risk factors for cerebrovascular disease are not disproportionately common in the patient group. In line with this, the MRI data reported in this chapter did not provide convincing evidence that macro or microvascular disease is more prevalent in TEA. $\mathrm{WMH}$ are the most widely studied radiological marker of small vessel disease in the brain, and were equally represented in both patient and control groups. There were subtle differences in the number of EPVS seen in the centrum semiovale and basal ganglia of TEA patients. However, the significance of this finding is far from clear since the clinical relevance of EPVS remains uncertain (Marnet et al., 2007). Recently, Jansen et al (Jansen et al., 2008) found no difference between WML load in a group of younger (age range 22 to 63 years) people with epilepsy and matched healthy controls. To my knowledge, no study has directly compared the incidence of WMH or EPVS in elderly people with epilepsy and carefully matched controls. It is important to note that the negative results reported in this chapter and in Chapter 2 do not rule out the possibility that subtle but strategically located cerebrovascular disease plays a role in the aetiology of TEA. Larger studies using more closely matched control populations and more sensitive imaging techniques are needed.

Volume reduction was demonstrated in the hippocampus of TEA patients using a manual delineation technique. Across the group, atrophy was detected in both left and right hippocampi. However, the overall degree of atrophy was subtle - about $8 \%$ of the total hippocampal volume. In this group of patients, it was not possible to distinguish electrophysiologically or radiologically between patients with a right or left-sided seizure focus. It may be, therefore, that averaging across the pathological and non-pathological hemispheres attenuated the observed volume differences. It cannot be assumed, however, that the pathology in TEA is as asymmetrical as that in other forms of TLE. Indeed, the semiology of TEA attacks, which is very similar to the classical amnesic syndrome, suggests that MTL dysfunction must be bilateral. It has been proposed that seizure activity in pure amnesic seizures might spread from one hippocampus to the other, without involving other brain regions, via the dorsal hippocampal commissure (Gloor et al., 1993). 
Using well-established anatomical landmarks to segment the hippocampus into head, body and tail regions, volume loss was found to be significant only in the hippocampal body. This contrasts with recent work employing similar methods in patients with medically intractable TLE, which found most atrophy in the hippocampal head (Bernasconi et al., 2003), although older findings include diffuse (Quigg et al., 1997; Van Paesschen et al., 1997) and focal hippocampal body (Bronen et al., 1995; Kuzniecky et al., 1996) atrophy. The connectivity of the hippocampus varies along its length, with the head receiving dense projections from the amygdala, entorhinal cortex and perirhinal cortex, whilst the major projections to the hippocampal body and tail arise from parahippocampal cortex (Witter and Wouterlood, 2002). Segmental atrophy in the hippocampus does not necessarily match the epileptogenic region (King et al., 1997), however, and the relation of our finding to the clinical phenotype of TEA is not yet clear.

No difference was detected in the size of the amygdala between TEA patients and control subjects. Although the anatomical boundaries of the amygdala are difficult to delineate on MR images (Pruessner et al., 2000), previous studies in medically refractory TLE have demonstrated amygdalar atrophy ipsilateral to the side of seizure onset (Bernasconi et al., 2003; Cendes et al., 1993; Watson et al., 1997). The atrophy is often, however, less marked than in the hippocampus.

Our VBM group analysis of patient versus control scans failed to confirm the manual finding of focal hippocampal atrophy. This is likely to be because the group differences are relatively subtle: the spatial normalization, tissue segmentation and smoothing components of image pre-processing in VBM inevitably lower the sensitivity of this method. A recent study of VBM in patients with Alzheimer's disease was able to detect approximately 20\% hippocampal volume reduction (as measured manually), at an uncorrected threshold of $\mathrm{p}<0.001$ (Acosta-Cabronero et al., 2008). The $8 \%$ reduction seen in TEA is probably below VBM resolution. Studies using VBM to investigate volume changes in TLE have usually focussed on patients with medically intractable epilepsy, often including those with hippocampal sclerosis. Such studies (Bernasconi et al., 2003; Bonilha et al., 2004; Bonilha et al., 2007b; Keller et al., 2002a; Mueller et al., 2006) have revealed grey matter loss in an extensive network including the ipsilateral and contralateral hippocampus, 
parahippocampal cortices, cingulate cortex, thalamus, insula, frontal lobes and cerebellum (for a review see Keller and Roberts (2008)), largely confirming the findings of manual volumetry. In patients with medically intractable seizures but no radiological evidence of hippocampal sclerosis, Mueller et al. (2006) recently failed to find evidence of grey matter volume loss using VBM, although a previous study with manual tracing revealed atrophy in the entorhinal cortex (Bernasconi et al., 2001).

Positive correlations were found between the volume of MTL structures and performance on standard anterograde memory tests using both manual volumetrics and VBM. Interestingly, right hippocampal volume was related specifically to performance on two tests of visuospatial memory - delayed recall of the Rey complex figure and face recognition memory. These results are consistent with the widely accepted notion that the hippocampus and surrounding cortices are crucially involved in the formation and at least initial retention of declarative memories, and that there is a degree of lateralisation between verbal and non-verbal memory (Kelley et al., 1998). Correlations of MTL atrophy with cognitive performance have previously been demonstrated in patients with TLE using manual volumetrics (Baxendale et al., 1998; Lencz et al., 1992; Reminger et al., 2004). VBM studies, however, have implicated much more extensive brain atrophy in the memory deficits of TLE (Bonilha et al., 2007a; Focke et al., 2008). Focke et al. (2008), for example, found global but no anatomically localised associations between grey matter volume and neuropsychological functions in 89 patients with unilateral hippocampal sclerosis. The findings of the present study indicate that TEA may involve more focal, if less marked, pathology.

No relation was found between the volume of medial temporal structures and ALF or autobiographical memory impairment. As discussed in Chapter 5, the pathophysiological mechanisms underlying ALF are not yet known, but there are several possibilities. Firstly, it might be simply a mild form of the amnesia typically associated with MTL damage. TEA patients did show slightly increased forgetting over 30 minutes although this was not predictive of very long-term recall for individual subjects (see Chapter 4 and Butler et al. (2007)). The failure to find structural correlates of ALF in this study may then be due to the use of insufficiently sensitive techniques. Secondly, ALF may be caused by intermittent neuronal 
dysfunction in the MTL. The close relationship between TEA attacks and waking from sleep raises the possibility that nocturnal, subclinical epileptic activity may disturb sleep-dependent memory consolidation processes (Stickgold, 2005). This possibility could be explored with prolonged EEG monitoring. Thirdly, ALF may be due to the effects of anticonvulsant medication. This is considered to be unlikely since patients i) are generally on low doses of anticonvulsants and ii) report that their memory improves on initiation of treatment. These explanations are not mutually exclusive and several factors may conjoin to produce ALF.

The cause of autobiographical memory loss in TEA is also unknown. Again, the results presented in this chapter indicate that it is not related to overt structural damage. A further possibility is that seizure activity leads to disruption of established memory traces (Manes et al., 2001). However, the mechanisms by which this might occur, and the brain regions involved are unclear. As discussed in Chapter 1, the neural substrate of remote memory is currently debated. The so-called 'standard theory of consolidation' holds that, following acquisition, all declarative memory traces gradually become independent of the hippocampus through a process of network reorganisation (Bayley et al., 2005; Squire and Alvarez, 1995). On the other hand, the 'multiple-trace theory' (Moscovitch and Nadel, 1998; Moscovitch et al., 2006; Nadel and Moscovitch, 1997) posits lifelong dependence of episodic memories upon the hippocampus. In TEA, the temporally extensive retrograde amnesia that occurs in the context of late-onset medial temporal lobe seizures lends some support to the multiple trace theory, though it is possible that seizure activity may propagate through the autobiographical memory network causing disruption outside the MTL.

Due to practical constraints, patients in this study underwent MR imaging in one of two centres. VBM has rarely been used to analyse data acquired from different scanners because of concerns about systematic bias arising from scanner and acquisition differences, particularly those affecting automated tissue segmentation. However, recent studies (Ewers et al., 2006; Stonnington et al., 2008) have suggested that variations from different scanners may have negligible effects on segmented grey matter images, particularly where the imaging platform is kept constant. In order to minimise systematic bias, the same model of scanner and acquisition protocol was 
used to image a mixture of patients and control subjects at both centres. Furthermore, the scanning centre was included as a covariate of no interest in the design matrices.

In the VBM group analysis, patients appeared to have increased grey matter volumes in the inferomedial temporal cortex - the inferior temporal gyrus, fusiform gyrus and parahippocampal gyrus - bilaterally. Similar findings have been reported in VBM studies of TLE patients (Bernasconi et al., 2004; Bonilha et al., 2004; Woermann et al., 1999), and are likely to be due to diminished grey-white matter demarcation in the temporal lobe of patients and subsequent tissue segmentation anomalies. In contrast, manual volumetric analysis of parahippocampal cortex in TLE has revealed grey matter volume reduction (Bernasconi et al., 2003). The findings in the present study require further exploration by manual delineation of the more lateral temporal cortices. This work is now underway.

No correlation was identified between regional brain atrophy and the duration of epilepsy or frequency of seizures using manual volumetrics or VBM. The weakly positive correlation seen between manually traced left hippocampal volumes and the lifetime number of seizures was not supported by VBM and is likely to be spurious, driven by a small number of outlying results for which the estimated number of seizures may be inaccurate. Similar investigations in medically refractory TLE have yielded mixed results, with some authors reporting positive (using manual volumetrics: (Kalviainen et al., 1998; Salmenpera et al., 2001; Seidenberg et al., 2005; Tasch et al., 1999; Theodore et al., 1999; Van Paesschen et al., 1997); using VBM: (Bonilha et al., 2006; Keller et al., 2002b)) and others negative (using manual volumetry: (Jutila et al., 2001; Spanaki et al., 2000); using VBM: (Bernasconi et al., 2004)) results. Longitudinal analyses have demonstrated progressive volume loss in the MTL of patients with TLE (Bernasconi et al., 2005; Liu et al., 2003; Salmenpera et al., 2005). The negative results of the present study are perhaps unsurprising for several reasons, including the inherent lack of sensitivity of cross-sectional analysis, the reliance upon retrospective patient reports, the relatively small sample size and the subtle degree of MTL atrophy. 


\subsection{Summary}

In this chapter, it has been demonstrated that patients with TEA rarely have clinically apparent brain lesions underlying their seizures. Furthermore, there is no convincing neuroradiological evidence of an increase in cerebrovascular disease compared with control subjects. However, patients do have subtle volume loss in the hippocampus and show a mild deficit in performance on standard tests of anterograde memory. Medial temporal lobe volumes correlate with several measures of anterograde memory. However, as reported in Chapter 4, the interictal memory deficits described by patients relate more closely to ALF and autobiographical amnesia than to standard memory test performance. These novel forms of memory impairment are not clearly related to regional brain atrophy. Further studies using metabolic and functional imaging as well as electrophysiological measures may help to determine their pathophysiological origin and how they relate to current models of human memory.

The hippocampal atrophy detected in this study adds weight to the hypothesis that the amnesic episodes of TEA are due to focal medial temporal lobe seizures. Additional support comes from a small number of cases, one of which is presented in the following chapter, where neuroimaging around the time of recurrent amnesic attacks has shown transient abnormalities in the hippocampus. 
Chapter 6 


\section{CHAPTER SEVEN}

\section{Transient epileptic amnesia: a detailed case report with radiological localisation}

\subsection{The Case}

NR, a 54 year old, right-handed American university professor, contacted the TIME Project after concluding from a search of the Internet that he was likely to be suffering from transient epileptic amnesia (TEA). He had a four-year history of recurrent episodes of transient amnesia and a progressive decline in memory function, but no other significant past medical or psychiatric history.

\subsubsection{Amnesic episodes}

At the age of 50, NR experienced an episode of transient amnesia which began abruptly as he emerged from the shower at ten o'clock one morning. He could not recall recent events. Alone in the house and curious as to why he should be showering so late, he went out to his car and felt that the bonnet was warm, implying that he had already been out. Over the next hour, as he attempted to reorientate himself by reading the newspaper and checking emails, his memories gradually returned. $\mathrm{He}$ recalled that, earlier that morning whilst out jogging with friends, he had briefly felt dizzy and had noticed a strong, unpleasant smell that was not detected by others. Later that day, he recounted the details of the episode to his doctor. Physical examination, a CT head and neuropsychological assessment were unremarkable. NR was given a diagnosis of transient global amnesia and assured that recurrence was unlikely.

Over the following three years NR suffered six further amnesic attacks. All occurred upon waking and were characterised by retrograde amnesia for events of the past days or weeks. In one journal entry he notes:

"Woke up at 3.30am - had no idea where I was. After stumbling around to find a light switch, found that I was in a small room in the P... Hotel in Milan (Italy). Initially 
had no idea why I was here. Found documents in room with my itinerary...Gradually began to recall that I was on a trip to attend meetings in Milan and Lugano."

A subsequent episode at home was witnessed by his wife whose questions revealed that he had no recollection of events from the past week, including watching his daughter play hockey, going to the movies and conducting heated negotiations with a car salesman. Another attack occurred on Christmas morning:

$25^{\text {th }}$ December: "I woke up at around 6am and realized I was having another attack and had no idea what time of year it was. I looked at my watch and saw that the date was the $25^{\text {th }}$. (My watch displays the day but not the month.) In trying to figure out what the month might be, it occurred to me that if it was December then this must be Christmas morning, but that seemed impossible at the time. I didn't think it was wintertime and I couldn't recall any activities relating to Christmas that had happened recently. It was still dark so I couldn't see whether there were any leaves on the trees. The episode lasted for well over half an hour. It was around 6.45 when things started to come back to me and I realized that it was Christmas morning."

On no occasion did NR fail to recognise his wife or his house. Anterograde memory was relatively preserved: NR did not repetitively question his wife, and was subsequently able to remember and record in his journal many details of the attacks. Other cognitive capacities including perception, attention, language and executive function were not noticeably impaired. There were no observed automatisms or periods of unresponsiveness. Over 30 to 60 minutes, his memories would gradually return, although the details of recent events sometimes remained hazy.

NR was assessed on several occasions by a neurologist and neuropsychologist. They reported that "no organic aetiology" could be identified and that "depression and anxiety are likely to be contributing". However, a psychiatrist found no evidence of psychiatric disorder.

Three and a half years after his first attack, NR experienced a more prolonged amnesic episode that only partially resolved over two hours, and was followed by several days of "anxiety, mental confusion and quasi-hallucinations - like 
'Groundhog Day"'. He was admitted to hospital for investigations. The neurological examination was normal. On mini mental state examination he scored 27/30, consistently failing to recall three words after a brief delay. Routine blood tests, chest $\mathrm{X}$ ray, electrocardiography and head CT with angiography were unremarkable. CSF biochemistry was normal and no cells were detected. Electroencephalography (EEG), magnetic resonance imaging (MRI) and FDG-PET were performed during the symptomatic period. The EEG showed an increase in slow wave activity over the left fronto-temporal region but no frankly epileptiform features. The MRI revealed high signal and swelling in the hippocampus, most prominently in the left hemisphere, on fluid attenuated inversion recovery (FLAIR) sequences (Figure 7.1a). The PET scan showed dramatic and circumscribed hypermetabolism in the left anterior hippocampus (Figure 7.1b). NR's condition improved gradually over his 5-day hospital admission. A seizure disorder was considered the most likely cause of the acute episode and levetiracetam therapy was started. One month later, with no intervening acute episodes, the PET abnormalities had resolved (Figure 7.1c). Further investigations were unremarkable, including serum antibodies against cardiolipin, voltage-gated potassium channels, $\mathrm{P} / \mathrm{Q}$ and N-type calcium channels, neuronal nuclei (ANNA 1,2 and 3), Purkinje cells (antibody types 1,2, TR and S), amphiphysin, CRMP-5, thyroglobulin, microsomes, striated muscle and glutamic acid decarboxylase.

Despite the anticonvulsant medication, NR had a number of further amnesic episodes associated with abnormal olfactory perception ("I seemed to be able to smell the last few meals I had eaten, on the skin of my hands and face"). He was admitted for EEG videotelemetry. Hyperventilation provoked a brief $(<1$ minute $)$ period of unresponsiveness, which was accompanied by spikes over the left temporal region. The EEG quickly normalised but, on subsequent questioning, NR proved unable to recognise the EEG technicians and gave the year as 1989. Anticonvulsant treatment was changed to lamotrigine and NR has since had no further acute episodes of memory loss or confusion. 
a)

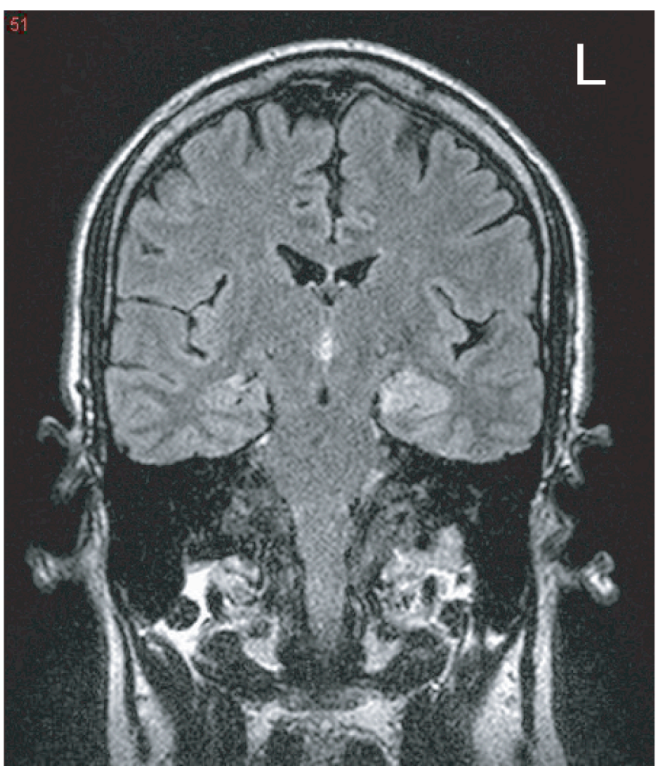

b)

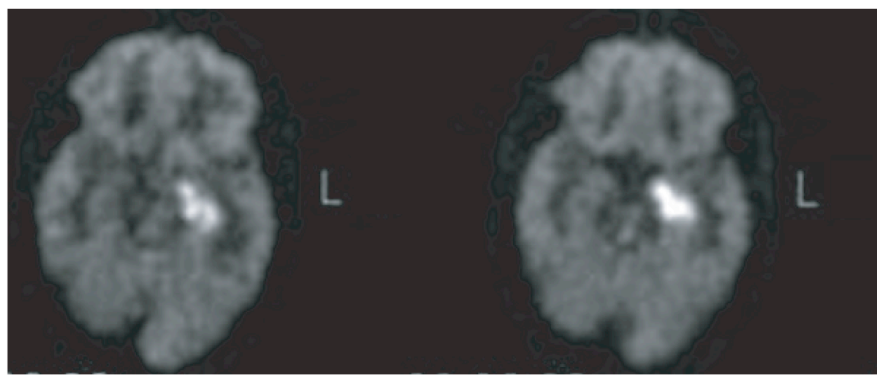

C)

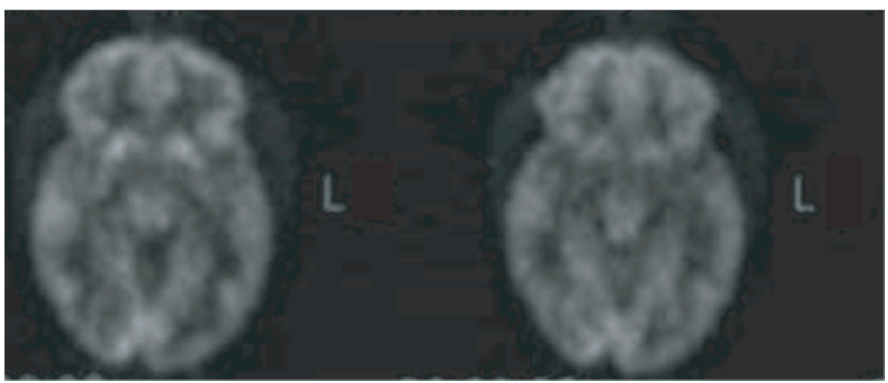

Figure 7.1: Brain imaging from NR. (a) Fluid-attenuated inversion-recovery (FLAIR) MRI scanning during a prolonged amnesic episode revealed hyperintensity in the left hippocampus. (b) 2-fluoro-2-[ $\left[{ }^{18} \mathrm{~F}\right]$-deoxy-D-glucose PET scanning during the same episode showed hypermetabolism localised to the left anterior hippocampus. (c) This region had returned to normal one month later.

\subsubsection{Persistent memory problems}

Coincident with the onset of the amnesic episodes, NR began to notice other more persistent memory problems. Firstly, he found that some newly acquired memories faded irredeemably over a period of days or weeks. For example, he went out to the cinema and the next day related his disappointment in the film to his daughter. However, one week later he was no longer able to recall anything of the evening's events. Three weeks after returning from the meeting in Milan during which he had an episode of transient amnesia, he notes:

"I remember almost nothing that went on at the meeting ... I don't remember any of the talks! I am also completely unable to remember anything about the conference banquet, even though I know there must have been a conference banquet somewhere 
at some point. I mentioned this to [his wife] and she told me that I had described a very elegant restaurant with grape vines as a roof over the outdoor terrace ... On the other hand, I seem to remember quite a lot about the meeting in Lugano which came afterward - lunches, dinners, poster sessions, etc."

One summer, he spent two weeks in Heidelberg and Munich. On his return, he gave a 2-hour presentation to his family of the photographs he had taken, accompanied by a detailed narrative. Six months later, however, he was unable to remember anything about the trip, to recognise the photographs, or to recall the slide presentation he had given. In his journal, NR described his memories “evaporating - it's as if random chunks of my memory have been erased". This loss of memories initially appeared limited to events post-dating the onset of the acute amnesic episodes. But NR gradually began to notice that his recollection of many remote, salient, personal events, such as family holidays and weddings from the past 20 to 30 years had become "very sketchy or completely absent". He wrote in his journal:

"I continue to notice "holes" in my memory which are scary but also becoming somewhat routine for me. I seem to have particular trouble remembering discrete events like movies and outings to restaurants and trips that I've taken, but meanwhile seem to be able to remember stable facts like my credit card number and my frequent flyer number."

"I remembered that I had some old pictures in a desk drawer. I pulled them out and looked through some of them - pictures of old camping trips, Christmas celebrations at home, etc. The scenes were familiar, but not as familiar as they should have been. Normally I would expect the sight of an old picture to awaken a specific memory of the event, tinged with emotion. This time the memories were very indistinct and the emotions very faint (except for a sense of loss!), as though I remembered the pictures but not the original events. It feels very strange when I write that I have lived here for 18 years-I don't seem to have anywhere near 18 years of memories at the moment."

NR also noticed early trouble with spatial memory. He complained that he was unable to visualise routes and navigate around previously familiar neighborhoods. For 
example, he retraced a favourite running route around local streets, but found that "hardly anything looked familiar". He frequently took wrong turns when driving alone and began to rely heavily upon internet-based map programs, even when heading to well known locations in the city.

Despite these difficulties, he continued with academic and teaching commitments, publishing eight papers as first author in the four years following the onset of his amnesic attacks and gaining a promotion from associate to full professor. Nevertheless, he commented that:

"My productivity is certainly affected by this, inasmuch as I also have trouble remembering of the contents of any papers I've read lately - or even papers that I have written! Reading my own work has become a lot like reading someone else's work, and I waste a good deal of time going back over the same ground."

The memory difficulties remained undetected by friends and colleagues, and even by a neuropsychologist who assessed him four times between 2002 and 2005 and found his performance to be exceptional in all cognitive domains despite the pronounced subjective complaints. 


\subsubsection{Neuropsychological assessment}

In September 2006, NR underwent a detailed neuropsychological assessment. His IQ was in the 'very superior' range. His performance on a variety of standard neuropsychological tests revealed well-preserved anterograde memory for both verbal and non-verbal material over a 30 minute delay, as well as normal language and executive function (see Figure 7.2).

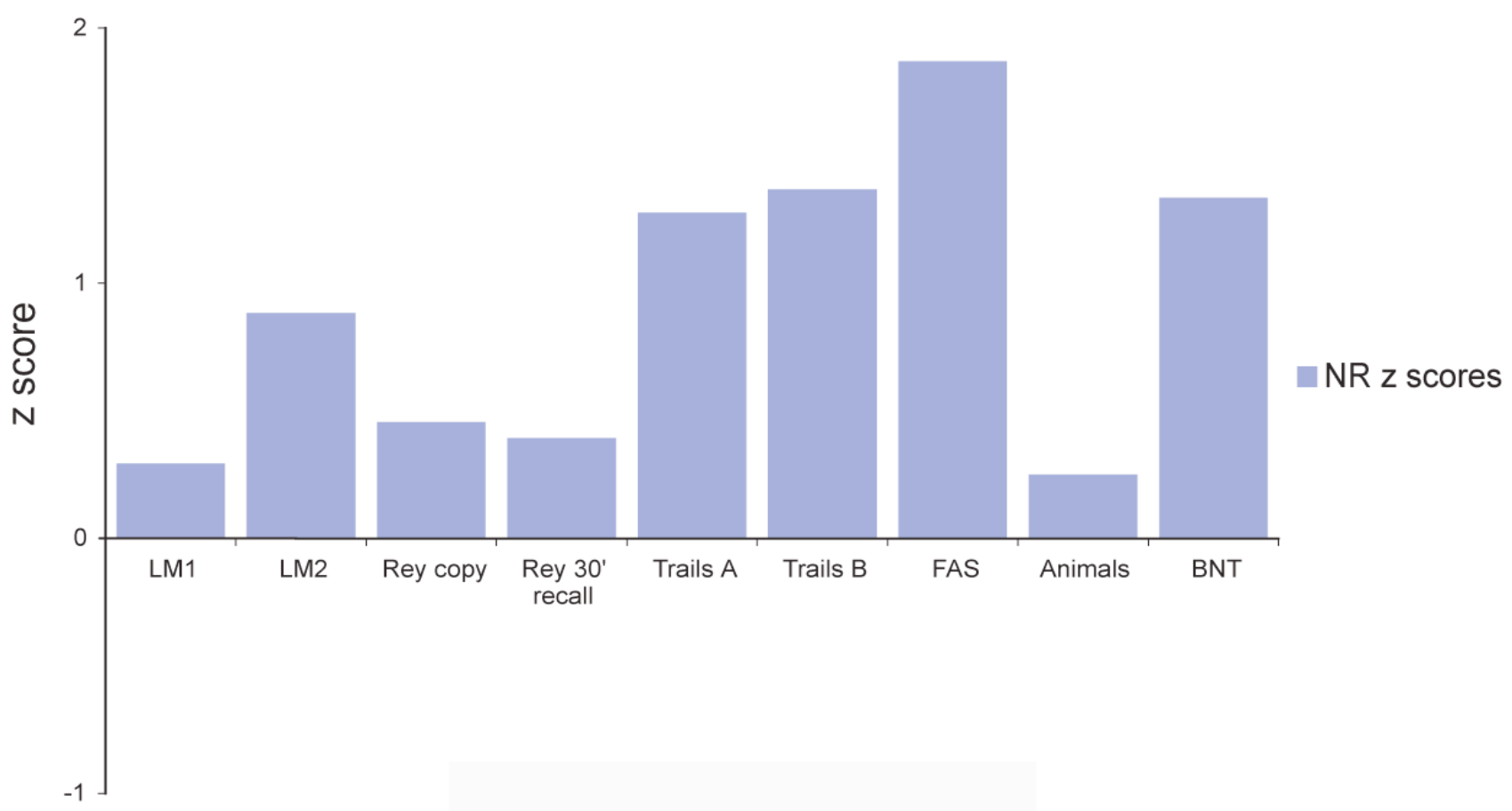

Figure 7.2: NR's performance on standard neuropsychological tests is shown as z scores against the control group from the TIME Project (mean age 67.7 years [SD 8.1]; mean IQ 120 [SD 14.4]). Memory tests: Logical Memory from the Wechsler Memory Scale III (LM1 and LM2) and copy and delayed recall of the Rey Figure. Executive function tests included the Trail-Making Test (Trails A and Trails B), and verbal fluency for letters (FAS) and categories (animals). The Boston Naming Test (BNT) was used to assess semantic memory. 
In order to investigate NR's complaint of memories “evaporating”, long-term verbal and non-verbal memory was tested over a period of three weeks using a short story and a set of seven simple designs (see Chapter 4 and Appendix G). The material was learned over repeated presentations until NR attained 90\% recall accuracy. Free recall was then tested at delays of 30 minutes, one week and three weeks. The results are shown in Figure 7.3, alongside those of a group of healthy controls (mean age $=67.7$ years $(\mathrm{SD}=8.1)$; mean $\mathrm{IQ}=120(\mathrm{SD}=14.4)$ ) (Butler et al., 2007). After 30 minutes, NR's recall was intact for both types of material. However, at one week and three weeks he recalled nothing of the story, and had even forgotten its having been administered. His recall of the designs was also significantly impaired.

(a)

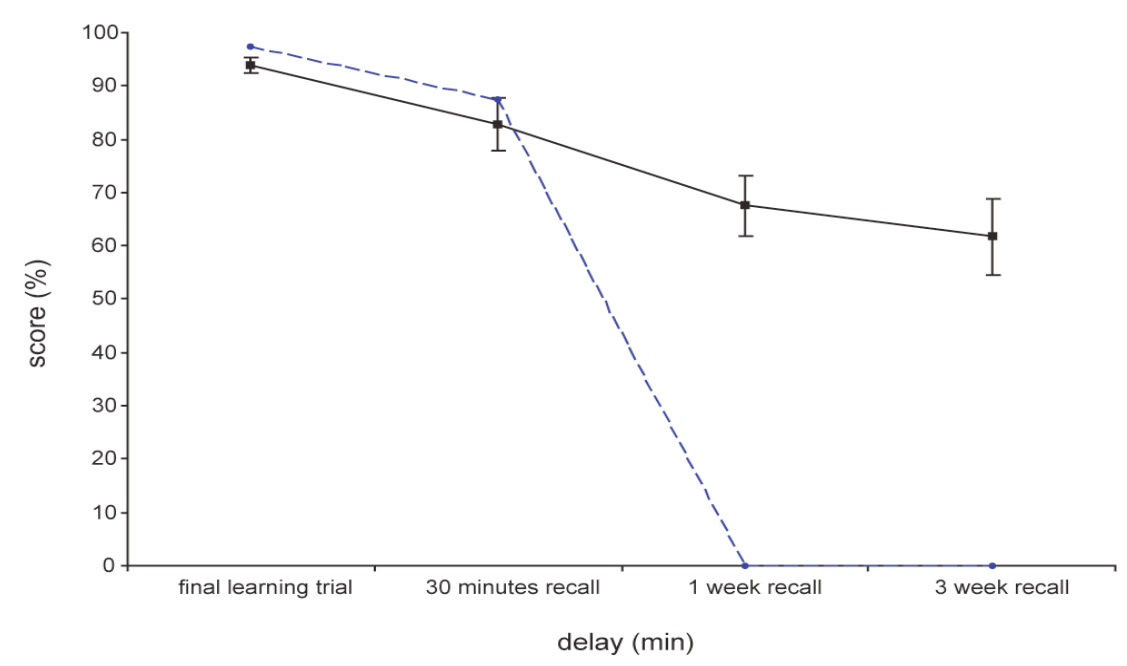

(b)

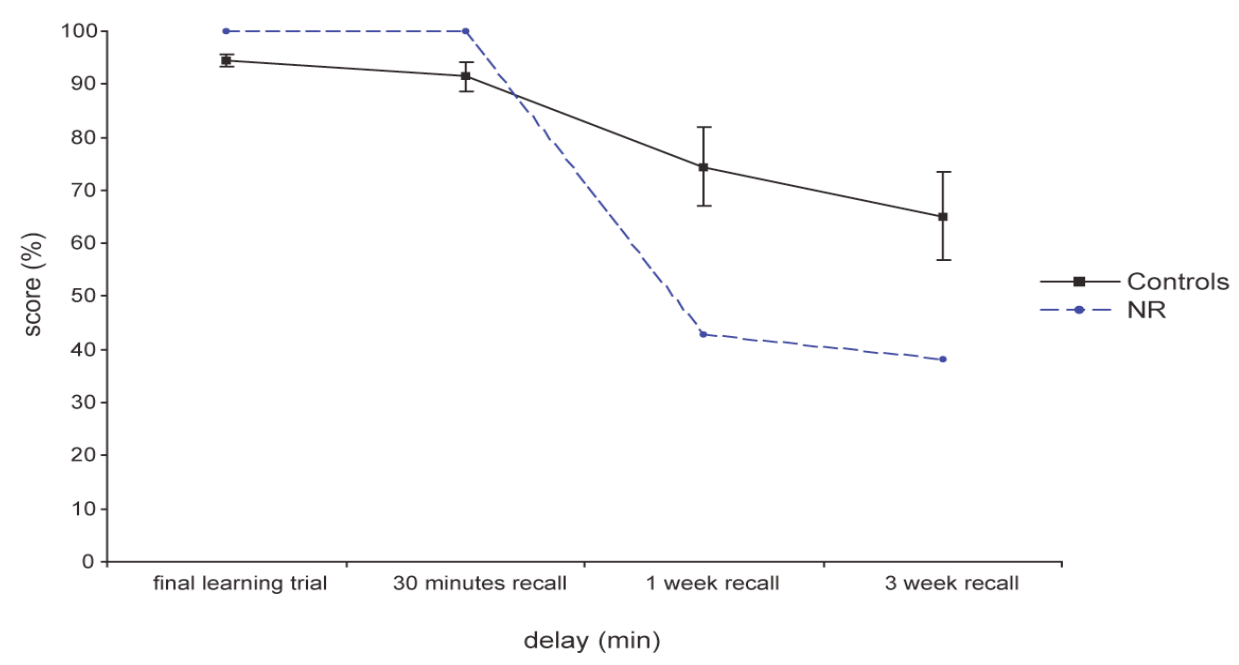

Figure 7.3: The patient's long-term recall of a learned (a) story and (b) set of designs was compared with performance of normal controls (error bars indicate 2 standard errors of the mean). Despite normal learning and 30-minute retention, he demonstrated accelerated forgetting over longer intervals. 
A semi-structured interview (the Modified Autobiographical Memory Interview (MAMI)) (see Chapter 4 and Appendix H) was used to assess memory for autobiographical events relating to two varying topics (e.g. 'holiday') for each decade of NR's life. For each topic, he was asked to produce one detailed episodic memory (e.g. "Can you recall any incident, even if small, that occurred during your holiday in San Diego?"). Figure 7.4 shows that, in comparison with the same control group, NR had a significant impairment of autobiographical memory, extending back over three decades but leaving older memories relatively intact.

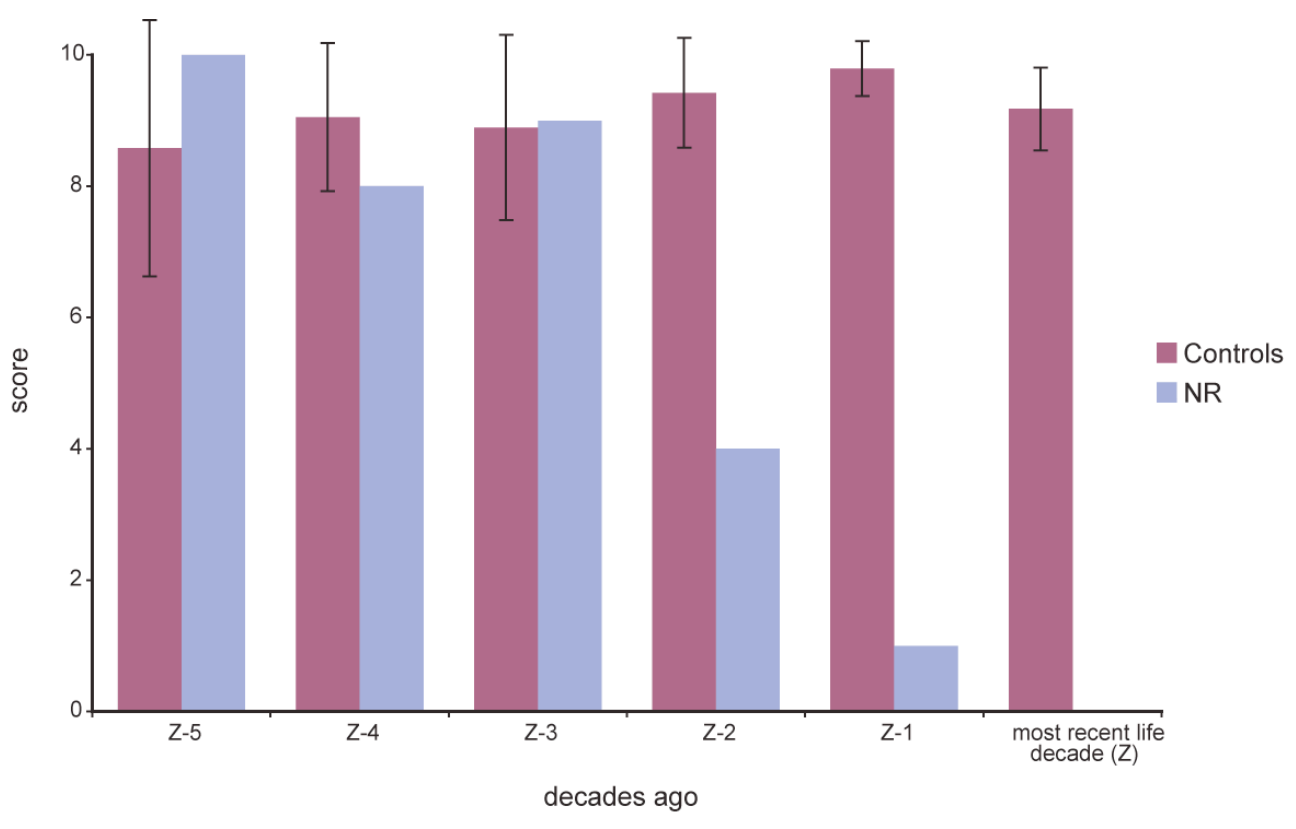

Figure 7.4: NR's performance on the episodic memory component of the MAMI (MAMI-e) compared with the TIME control group. His memory for events from the previous three decades was strikingly impaired. Error bars indicate two standard errors of the mean.

Despite these deficits, NR's knowledge of public facts and events was relatively spared. He was able to name all the US presidents of the past century and date the period that they held office. He was also able to name and date photographs of many of the most valuable baseball players of the past 50 years. 
NR underwent MR imaging of the brain at 4-tesla field strength. A single coronal image, alongside a 1.5-tesla scan from two years earlier, is shown in Figure 7.5. The later scan reveals new atrophy restricted to the left hippocampus.

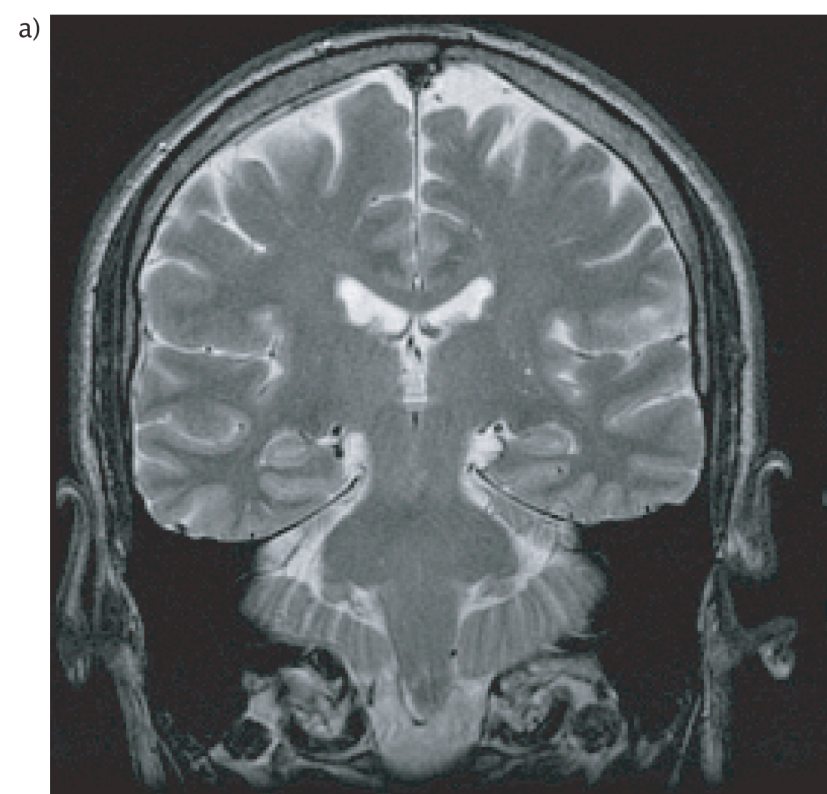

CA3

c)

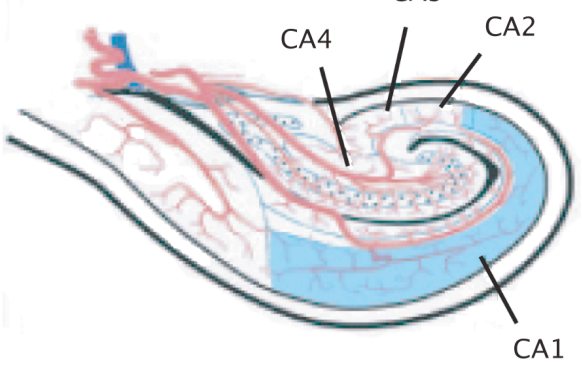

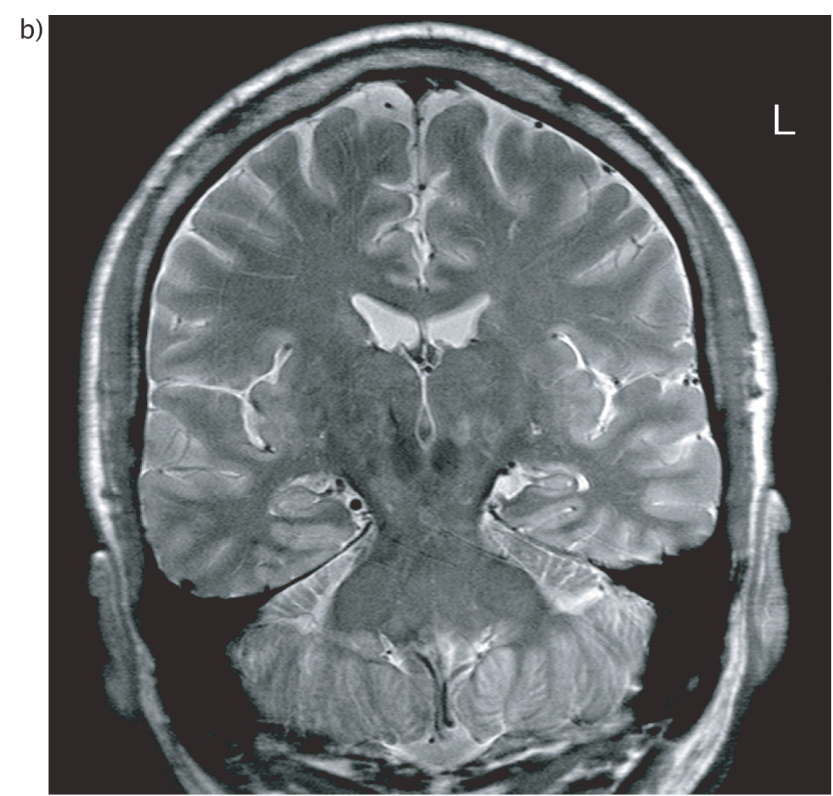

d)

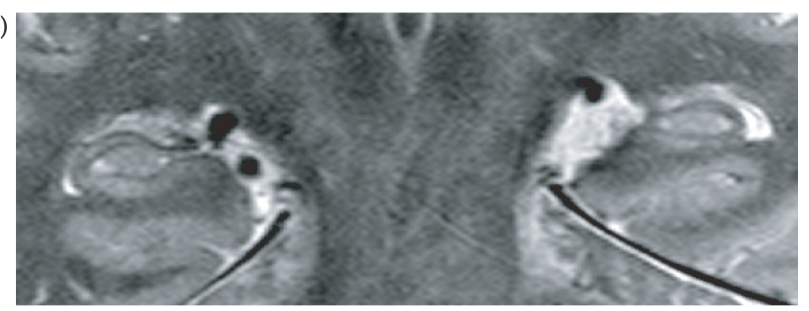

Figure 7.5: T2-weighted structural brain imaging from NR. a) 1.5-Tesla coronal section from 2004, two years after the onset of his amnesic attacks. The hippocampi are of normal appearance. b) 4-Tesla fast spin-echo MRI sequence in 2006, several months after his prolonged amnesic episode. The scan was acquired at the time of detailed neuropsychological testing. Focal atrophy of the left hippocampus is seen. c) Sketch of the left hippocampus illustrating subfields. d) Higher magnification view of the medial temporal lobes in 2006 . 


\subsection{Discussion}

This case illustrates many of the characteristic features of TEA and provides convincing radiological evidence of a seizure focus in the hippocampus, a brain region intimately involved with memory. It also highlights the increasing use of the Internet by patients with unusual disorders in pursuit of their diagnosis.

NR fulfils all the diagnostic criteria for TEA. He experienced recurrent episodes of isolated memory loss during which other cognitive functions - for example, his ability to converse, to attempt to orientate himself by checking emails and to write in his journal - were preserved. There is substantial evidence for a diagnosis of epilepsy, including the epileptiform discharges seen on video telemetry, the co-occurrence of olfactory hallucinations and the cessation of the amnesic attacks with anticonvulsant medication. Furthermore, NR exhibits many other typical features. His attacks began in middle age. They almost invariably occurred upon waking, generally lasted about 30 minutes and were characterised by a retrograde amnesia but a striking preservation of anterograde memory. He complained of marked persistent memory impairment including i) the 'evaporation' of new memories over days to weeks - accelerated long-term forgetting (ALF), ii) 'holes' in his memory for events of the past 20 to 30 years - remote autobiographical amnesia, and iii) new difficulty finding his way around previously familiar environments - topographical amnesia. Despite this, he performed at a high level on standard neuropsychological testing, and continued with his teaching and research commitments. His interictal memory impairment was demonstrated with the novel instruments designed for the TIME Project.

The case of NR illustrates the fact that TEA is under-recognised and often misdiagnosed by neurologists. Over three and a half years, he experienced seven typical episodes of TEA that were labelled as 'psychogenic'. Such delays in diagnosis are important for several reasons. Firstly, instigation of the correct treatment usually results in cessation of the amnesic episodes. Secondly, misdiagnosis and inappropriate medication may have significant negative consequences for the patient. Thirdly, as demonstrated by the seizure captured on videotelemetry, TEA episodes may be associated with periods of altered awareness and diminished responsiveness, a finding that has important implications for safety and, in particular, driving. Fourthly, as discussed below, recurrent seizure activity, especially status epilepticus, may have 
long-term consequences for neuronal integrity and memory function. This possibility is yet to be systematically explored in TEA. However, the journal entries of NR illustrate a sentiment often expressed by patients: that autobiographical memory gaps appear after periods of frequent seizures and that long-term memory retention improves when anticonvulsant medication is started. It is possible, therefore, that early treatment may limit the development of persistent memory deficits.

The amnesic episodes experienced by NR demonstrate that anterograde amnesia may be incomplete during TEA attacks: he was able subsequently to record considerable detail about them in his diary. Vuilleumier et al. (1996) report the case of a 41-year old woman who experienced an amnesic attack lasting several hours. During the episode, ongoing ictal activity was observed over bilateral frontotemporal regions on the EEG. Ictal neuropsychological evaluation revealed a mixed anterograde and retrograde amnesia that later resolved. After the attack, however, she was able to give detailed information about events that had occurred during it, including the neuropsychological testing. The authors interpret this as a transient, but dense, impairment of memory retrieval mechanisms in the context of preserved memory encoding. The partial preservation of anterograde memory often found during TEA attacks distinguishes the condition from TGA, following which patients always have a dense amnesic 'gap' for the episode itself (Hodges, 1991). The reasons for this difference are unclear. It may reflect a difference in the degree, anatomical distribution or underlying mechanism of neuronal dysfunction. Besides being a useful diagnostic clue, therefore, this phenomenon deserves future attention in the quest towards understanding how epilepsy disrupts memory networks.

NR first noticed persistent memory difficulties at around the same time as the onset of his amnesic episodes. These difficulties are typical of those found in TEA - ALF, autobiographical memory impairment and topographical amnesia. His subjective impression is that these difficulties were progressive and had already become profound before his prolonged amnesic attack. As with many TEA patients, NR's performance on standard neuropsychological measures, even taking into account his presumably very high premorbid baseline, appears to be intact. However, he showed marked accelerated forgetting of verbal and non-verbal material over extended delays. The fact that this problem also affected 'real life' memories was illustrated by the fact 
that, one week after I had spoken to him on the telephone for the first time, a conversation which had lasted for over a hour, he had no recollection of having talked with me before. Furthermore, his performance on the MAMI revealed a profound autobiographical amnesia for events of the three decades. There was an apparent preservation of memories formed during the first 30 years of his life. This 'Ribot gradient' for autobiographical memories is commonly observed in cases of partial hippocampal damage, and is directly predicted by both the standard theory of consolidation (STC) and the multiple trace theory (MTT) (see Chapter 1). However, the temporal extent of the gradient in this case is remarkable. If the neuronal dysfunction associated with TEA were restricted to the medial temporal lobes, then the STC would have to postulate a consolidation process, by which memory traces become independent of the hippocampus, that lasts for decades. A more plausible proposal might be that functional disruption in TEA extends into regions associated with permanent storage of episodic memory traces, such as the temporal neocortex. The MTT, on the other hand, would expect medial temporal lobe seizures to disrupt memories from across the lifespan, but might predict that older memories, supported by multiple hippocampal traces, would be relatively preserved. The reported patchiness of the autobiographical memory loss in TEA, which is yet to be clearly documented, is difficult to explain. Further work is needed to determine which, if either, of the currently available models best fits with the persistent memory deficits seen in TEA.

The nature of the amnesia during attacks of TEA, the frequency of olfactory hallucinosis and the location of interictal epileptiform discharges on EEG suggest that the seizure focus lies in the medial temporal lobes, a brain region intimately involved with declarative memory (Squire et al., 2004). The case of NR, in whom FDG-PET scanning during a prolonged period of amnesia showed focal hypermetabolism in the left anterior hippocampus, supports this hypothesis. Ictal PET is rarely performed in patients with epilepsy due to practical constraints, most notably the short half-life of positron emitting isotopes such as ${ }^{18} \mathrm{~F}$. In the majority of reported instances, ictal FDG-PET scanning has revealed increased isotope uptake, reflecting hypermetabolism, in the region of the seizure focus as identified by EEG (Chugani et al., 1993; Fong and Delgado-Escueta, 1999; Meltzer et al., 2000), although one case study found ictal hypometabolism (Chung et al., 2002). Ictal single photon emission 
computed tomography (SPECT) is a more practicable, and hence more widely used, functional imaging technique and provides a measure of regional brain perfusion using technetium-based tracers, which have a longer half-life than ${ }^{18} \mathrm{~F}$. As with FDGPET, there is increased tracer uptake at the seizure focus, as well as in regions to which seizure activity propagates (van Paesschen, 2004). In contrast, interictal FDGPET scanning often reveals hypometabolism in the region of, and beyond, the seizure focus (Newberg and Alavi, 2005). This technique is sometimes useful in the presurgical evaluation of patients with epilepsy, particularly those in whom structural neuroimaging is inconclusive (Carne et al., 2004; Uijl et al., 2007). It seems likely that the PET data from NR were collected during a period of frequent or continuous focal seizure activity - nonconvulsive status epilepticus - despite the absence of clear epileptiform abnormalities on the surface EEG. The full PET dataset from NR is unfortunately unavailable. It would otherwise have been interesting to quantify regional variations in metabolism, and thus identify any evidence of seizure propagation beyond the left hippocampus.

In addition to the PET findings, the MRI scan performed during NR's prolonged period of amnesia revealed high T2 signal predominantly in the left but also in the right hippocampus. Ictal T2 hyperintensities have been reported in nonconvulsive status epilepticus, and are thought to represent vasogenic and cytotoxic oedema at the seizure focus (Cox et al., 1995; Henry et al., 1994; Lee et al., 1992). Lee et al. (1992) describe a case of prolonged transient amnesia, similar to that of NR, in which the EEG revealed frequent bursts of left mesiotemporal ictal activity. Periictal MRI in this case also showed T2 hyperintensity bilaterally in the medial temporal lobes, an abnormality which later resolved. A similar radiological pattern was seen in a TIME patient (case 116), who had an additional clinical MRI scan two years after his research assessments, during a period of recurrent amnesic spells and interictal memory deterioration. The bilateral hippocampal high signal can be seen in Figure 7.6. 


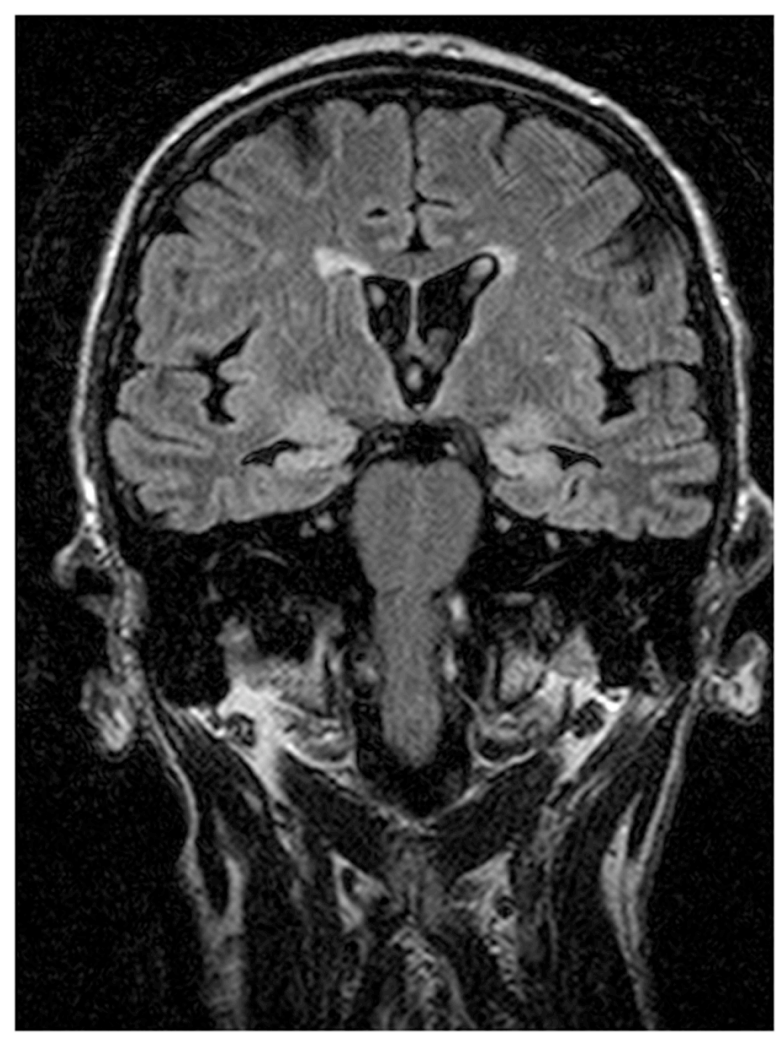

Figure 7.6: Coronal FLAIR image from TIME case 116 during a period of frequent amnesic episodes. High signal can be seen in the medial temporal lobes bilaterally.

The transient MRI abnormalities observed in these three cases are in keeping with the hypothesis that bilateral hippocampal dysfunction may underlie the amnesic episodes of TEA, although this needs to be investigated in a larger group of patients, perhaps using combined functional imaging and EEG (see Chapter 8).

As in the majority of TEA patients (Butler et al., 2007), NR's structural MR scans were initially normal. However, following the prolonged amnesic episode, volume loss became apparent in the left hippocampus. It is well recognised that nonconvulsive status epilepticus can cause permanent structural changes, including neuronal death and neurogenesis, in the hippocampus (Sutula et al., 2003; Walker et al., 2005). Most evidence comes from animal models (Krsek et al., 2004; Meldrum and Brierley, 1973) and suggests that excess glutamate release results in a chain of cytotoxic events that involves NMDA-receptor activation, raised intracellular calcium, mitochondrial dysfunction and subsequent oxidative damage. In humans, a few case studies have shown transient T2 and diffusion-weighted imaging changes with subsequent regional atrophy (Chu et al., 2001; Lansberg et al., 1999; Lazeyras et 
al., 2000), whilst DeGiorgio et al (1992) found hippocampal cell loss in pathological specimens from a group of five patients who died following status epilepticus compared with other patients with epilepsy and normal controls. The possibility that seizures outside the context of status epilepticus result in permanent neuronal damage is more controversial (Sutula et al., 2003). In the case of NR, longitudinal measurement of the volume of medial temporal and other brain regions was not possible due to inconsistencies in the scanning sequences used. In future, this sort of analysis will be important in identifying long-term structural changes associated with TEA and their relationship with neuropsychological measures.

\subsection{Summary}

This case illustrates many of the typical features of TEA as well as providing convincing radiological evidence that the syndrome results from seizure activity in the hippocampus. It also highlights important questions for future research. These questions, and a number of proposals for how they may be answered, are discussed in detail in the final chapter. 


\section{CHAPTER EIGHT}

\section{General Discussion}

\subsection{Introduction}

In this thesis, I have described the clinical, neuropsychological and radiological characteristics of transient epileptic amnesia (TEA), based on a detailed study of 50 patients from around the United Kingdom - the TIME (The Impairment of Memory in Epilepsy) Project.

Whilst it has been recognised for over a century that transient episodes of amnesia may be the principle manifestation of epilepsy (Hughlings-Jackson, 1888), the relevant scientific literature is sparse. The diagnosis is often missed by clinicians (Kosmidisand and Papanicolaou, 2006; Mendes, 2002). Discussions (Gallassi, 2006; Gallassi et al., 1986; Kapur, 1993b; Zeman et al., 1998) have suggested that patients with this form of epilepsy have many features in common including their age, the clinical characteristics of the amnesic attacks and associated complaints of unusual interictal memory difficulties - in particular, accelerated long-term forgetting (ALF) and autobiographical memory loss. However, to date there has been no large, systematic study of TEA. The aim of the work presented in this thesis was to perform such a study and provide a detailed, authoritative description of the condition. A further objective was to document the phenomena of ALF and autobiographical amnesia in TEA and explore their relation to seizure variables and brain structure. In this final chapter, I begin by summarising the principle findings of the study. I then highlight a number of outstanding questions and propose some directions for future study. 


\subsection{Principle findings of the study}

\subsubsection{Clinical features of TEA}

TEA is defined by recurrent, transient episodes of isolated amnesia for which there is convincing evidence of an epileptic origin. The results presented in Chapter 2 show that it is under-recognised by clinicians. The median delay from the first amnesic attack to diagnosis among patients in this study was 12 months. Only one quarter of patients were correctly diagnosed with temporal lobe epilepsy upon their initial presentation to a specialist physician. TEA typically begins in late middle age with a mean age of onset of 62 years and an age range of 44 to 77 years in this study. It appears to be about twice as common in men than women. The amnesic attacks often occur upon waking and occur exclusively in this context in approximately one quarter of patients. There is a variable combination of anterograde and retrograde amnesia, but the anterograde component is often incomplete so that about $50 \%$ of patients report 'remembering not having been able to remember'. During attacks, patients remain able to respond appropriately to conversation and act in a purposeful manner, testifying to their otherwise preserved cognitive abilities. Other features of epilepsy may co-occur with the amnesia - most commonly, hallucinations of smell or taste in up to $50 \%$ of patients. The attacks typically last around 30 minutes to one hour. However, much longer episodes, even persisting for several days, were reported, and these may reflect persistent seizure activity - nonconvulsive status epilepticus. Episodes of memory loss recur with an average frequency of around one per month. EEG reveals epileptiform abnormalities in approximately one third of cases, especially after sleep deprivation, and these are usually seen over temporal regions. In common with other forms of late-onset epilepsy, TEA generally responds well to a low dose of anticonvulsant medication. Previous work has suggested a link between TEA and cerebrovascular disease (Zeman et al., 1998). However, in this study, analysis of cerebrovascular disease risk factors and brain imaging data did not support this hypothesis. The comprehensive review presented in Chapter 3 revealed that the features demonstrated by the TIME cases are broadly consistent with those of other cases in the literature. 
There are a number of similarities between TEA and transient global amnesia (TGA) and the two can often be indistinguishable at first, even by experienced clinicians using strict diagnostic criteria (Hodges and Warlow, 1990a). Another common misdiagnosis, given in $18 \%$ of patients in the TIME Project, is 'psychogenic' or 'functional' amnesia. The principle clinical features that distinguish TEA, TGA and psychogenic amnesia are shown in Table 8.1.

\section{Table 8.1: Distinguishing clinical features of the transient amnesic syndromes}

\begin{tabular}{|c|c|c|c|}
\hline & Transient Global Amnesia & Transient Epileptic Amnesia & Psychogenic Amnesia \\
\hline typical age & 50 to 70 years & 50 to 70 years & all ages \\
\hline $\begin{array}{l}\text { past medical } \\
\text { history }\end{array}$ & migraine & nil & $\begin{array}{l}\text { 'organic' transient amnesia, } \\
\text { substance abuse, psychiatric } \\
\text { illness }\end{array}$ \\
\hline precipitants & $\begin{array}{l}\text { cold water, physical exertion, } \\
\text { psychological stress }\end{array}$ & waking & $\begin{array}{l}\text { minor head injury, stress, } \\
\text { depression }\end{array}$ \\
\hline $\begin{array}{l}\text { ictal memory } \\
\text { profile }\end{array}$ & $\begin{array}{l}\text { profound anterograde amnesia } \\
\text { with repetitive questioning; } \\
\text { variable retrograde amnesia; } \\
\text { non-declarative memory intact }\end{array}$ & $\begin{array}{l}\text { variable anterograde and } \\
\text { retrograde amnesia (may later } \\
\text { partially recall attack); non- } \\
\text { declarative recall apparently intact }\end{array}$ & $\begin{array}{l}\text { highly variable: often profound } \\
\text { retrograde amnesia with loss of } \\
\text { personal identity; relatively } \\
\text { preserved anterograde memory; } \\
\text { procedural memory may be } \\
\text { impaired }\end{array}$ \\
\hline other features & $\begin{array}{l}\text { headache/nausea may be } \\
\text { present }\end{array}$ & $\begin{array}{l}\text { olfactory hallucinations; } \\
\text { oroalimentary automatisms; brief } \\
\text { loss of responsiveness }\end{array}$ & $\begin{array}{l}\text { focal 'neurological' symptoms or } \\
\text { signs, e.g. hemiparesis, may be } \\
\text { present }\end{array}$ \\
\hline duration & typically 4-10 hours & $\begin{array}{l}\text { usually }<1 \text { hour but may last much } \\
\text { longer (days) }\end{array}$ & days or months \\
\hline recurrence & rare & mean frequency $=13 /$ year & rare \\
\hline $\begin{array}{l}\text { postictall } \\
\text { interictal } \\
\text { memory }\end{array}$ & $\begin{array}{l}\text { grossly intact, but subtle } \\
\text { deficits may persist for months }\end{array}$ & $\begin{array}{l}\text { accelerated forgetting, remote } \\
\text { autobiographical memory loss and } \\
\text { topographical amnesia }\end{array}$ & $\begin{array}{l}\text { variable: may 'relearn' the past } \\
\text { causing memories to lack } \\
\text { 'autonoetic consciousness' }\end{array}$ \\
\hline
\end{tabular}

\subsubsection{Neuropsychological features of TEA}

Even after complete cessation of the acute amnesic attacks with anticonvulsant medication, patients with TEA often complain of ongoing memory difficulties. Such difficulties were reported by three quarters of patients in the TIME Project. Seventy percent described a patchy loss of autobiographical memory, often for events that occurred several decades previously. Fourty-four percent reported the symptoms of 
ALF - the excessively rapid fading of newly acquired information over days to weeks. Furthermore, 36\% of patients described topographical amnesia. Subjective complaints of persistent memory problems were reflected in responses to two selfcompletion questionnaires - the Everyday Memory Questionnaire (EMQ) and the Very Long Term Memory Questionnaire (VTLMQ) - in which patients rated their memory for day-to-day facts and events as well as remote autobiographical events significantly worse than control subjects.

The data reported in Chapter 4 show that performance on standard neuropsychological tests is generally normal or only mildly impaired relative to control subjects. The patient group as a whole attained mean scores approximately one standard deviation below those of the control group on several tests of verbal and non-verbal anterograde memory including delayed story recall, delayed recall of the Rey complex figure, and the Recognition Memory Test for words and faces. However, these deficits did not correlate with subjective memory deficits as captured by the EMQ and VTLMQ. Patients were unimpaired on tests of language, semantic memory, visuospatial perception and executive function.

In a subgroup of 24 patients with no evidence of impairment on standard anterograde memory tests, accelerated forgetting of newly acquired information was demonstrated for both verbal and non-verbal material after a delay of three weeks. Patients showed no evidence of impairment in learning, reaching a difficult criterion of $90 \%$ correct in the same number of trials as control subjects. Excessive forgetting was demonstrable amongst patients after a 30-minute delay, but this was subtle. A much more dramatic difference in recall was found between the groups after the one-week and three-week delays. Recall performance after extended delays was consonant with patients' subjective impression of interictal memory impairment: it correlates with scores on the EMQ and was significantly different in the groups of patients who did and did not complain of ALF in everyday life.

The same subgroup of patients also demonstrated significant impairment on a new test of autobiographical memory - the Modified Autobiographical Memory Test (MAMI). Deficits were shown in memory for both personal facts and personally experienced events of the past 30 to 40 years. Performance on the MAMI reflected 
patients' subjective experience of remote memory deficits as detected by the VTLMQ. Moreover, in some patients the autobiographical memory impairment was present in the context of entirely normal anterograde memory, even when measured over extended delays. These patients therefore demonstrate the controversial phenomenon of 'focal retrograde amnesia' (Kapur, 1993a; Kapur, 2000; Kopelman, 2000).

As discussed in Chapter 5, ALF and remote memory impairment have also been shown to exist in patients with other forms of epilepsy. These phenomena may go some way towards explaining the widespread but poorly understood complaints of memory impairment amongst such patients. However, investigations of long-term forgetting and remote memory in epilepsy encounter a number of methodological complications, and no consensus has yet been reached on how best to overcome them. Patients with TEA, who have late-onset epilepsy in which seizures are relatively responsive to low doses of anticonvulsant medication, provide a useful context in which to investigate these phenomena.

\subsubsection{Neuroimaging features of TEA}

Structural brain imaging in TEA is usually unremarkable. Only two patients in this study had focal structural abnormalities potentially related to their epilepsy: one a petrous ridge meningioma, the other bilateral temporal neocortical atrophy. Nevertheless, manual volumetry revealed bilateral hippocampal atrophy in the patient group. This atrophy was subtle, representing a decrease of approximately eight percent in total volume, and was present only in the hippocampal body. No volume difference was detected in the amygdala. It is unclear whether the hippocampal atrophy reflects the pathology underlying the epilepsy or whether it is a consequence of seizures.

Using both manual volumetrics and voxel-based morphometry (VBM), the hippocampal atrophy was shown to be related to a decline in performance on standard tests of anterograde memory. In comparison with other studies investigating the neuroanatomical correlates of cognitive dysfunction in epilepsy, the VBM findings in 
TEA suggest a more focal pathology centred on the hippocampus and parahippocampal cortices. In contrast, no structural correlates of ALF and RMI, the most striking interictal memory deficits in TEA, were found. The lack of any evident correlation of these unusual memory deficits to brain structure, or to performance on standard neuropsychological tests, is consistent with the possibility that they are cognitively and pathophysiologically distinct. However, this issue requires further exploration as discussed below.

\subsubsection{The syndrome of TEA}

A medical syndrome is a 'distinct group of symptoms and signs which, when associated together, form a characteristic clinical picture or entity' (Panayiotopoulos, 2002). In the light of the findings presented in this thesis, I propose that TEA represents a distinctive but under-recognised syndrome characterised by the features described above. These features provide preliminary grounds for regarding TEA as an 'epilepsy syndrome' as defined by the International League Against Epilepsy (Engel, 2001). Syndromes are heuristic devices and, as such, map imperfectly onto clinical reality. A degree of blurring around the edges is therefore to be expected. During the course of patient recruitment for the TIME Project, I encountered a number of cases in which precise classification was impossible. Three such patients are described in Appendix D. The dilemma is illustrated by the conceptual proximity to TEA of the 'epileptic amnesic syndrome' (Gallassi, 2006) and 'pure amnestic seizures' (Palmini et al., 1992), both of which were discussed in Chapter 1. Whereas these ideas arose directly from the study of temporal lobe epilepsy, the concept of TEA evolved from work on TGA and emphasises the similarities and distinctiveness of TEA from that syndrome.

\subsection{Outstanding questions and suggestions for future work}

The results of the TIME Project define the general characteristics of TEA. However, there are many outstanding questions both with regard to the clinical features of the syndrome and its theoretical implications for the neuroscience of human memory. These questions, together with some proposals for how they may be addressed in future work, are discussed in the following section. 


\subsubsection{Clinical features of TEA}

\section{Aetiology}

The aetiology of TEA remains unclear. Whilst previous work suggested that cerebrovascular disease might be responsible (Zeman et al., 1998), this hypothesis was not supported by analysis of vascular risk factors and neuroimaging in the TIME Project. It is possible, however, that our sample size was too small, the control group insufficiently well matched and the imaging techniques too insensitive to detect the relevant differences. Cerebrovascular disease is a plausible trigger for TEA given the age range of patients and the location of the hippocampus at a vascular 'watershed'. Alternatives include a form of mild limbic encephalitis or a primary neurodegenerative process. It is possible that the syndrome can accompany a range of brain pathologies. Long-term follow up of a large cohort of patients, more sensitive neuroimaging techniques and post-mortem study of brain tissue should help to identify the cause of TEA.

\section{Epidemiology}

TEA is generally believed to be a rare condition. Given that the incidence of TGA is believed to be about 10 per 100,000 (Hodges, 1991), and that Hodges and Warlow (1990b) identified eight patients amongst their series of 114 TGA cases who later developed epilepsy, a minimum estimation can be placed at around one per 100,000. However, it is expected that patients meeting the diagnostic criteria for TEA are much more common than this. The high average IQ of the cohort is likely to reflect ascertainment bias. The amnesic episodes may often, at least initially, be attributed to 'old age' by patients, and a clear history is sometimes difficult to elicit, especially when the clinician is unfamiliar with the features of TEA. These facts help explain the diagnostic delay experienced by many patients and suggest that many cases remain undiagnosed.

\section{Amnesic episodes}

All of the data regarding the amnesic episodes in this study, and the vast majority in the literature, are based on accounts of patients and eyewitnesses. The overall 
impression of a typically mixed anterograde and retrograde amnesia, in the context of otherwise preserved cognitive functioning, is likely to be just an approximation to the truth. There are obviously considerable practical difficulties in performing detailed neuropsychological testing during episodes of transient amnesia, particularly in a study such as this in which most patients were already on anticonvulsant medication. Nevertheless, it will be important to design studies in which such testing is possible if the pathophysiological basis of the syndrome is to be understood. It may be, for example, that subtypes of TEA emerge, classifiable by the relative density of the anterograde and retrograde amnesia or by whether the amnesia is ictal or post-ictal. Video-telemetry of patients with frequent amnesic episodes will be a useful tool in this regard.

\section{Source localisation}

Given the apparent similarity between TEA attacks and the amnesia observed in TGA and other forms of medial temporal lobe amnesia, it is expected that TEA results from disruption of function in the hippocampus and related structures. Certain clinical features, including the frequent co-occurrence of olfactory hallucinations and the predominance of temporal lobe epileptiform abnormalities on the EEG, are consistent with this hypothesis. The focal medial temporal hypermetabolism seen on peri-ictal PET imaging of the patient described in Chapter 7, and the hippocampal oedema seen on MR scanning of this and other patients (see Chapter 7 discussion), add further support. The existence of a medial temporal seizure focus cannot be verified with surface EEG technology alone. However, concurrent capture of EEG and fMRI data from patients with frequent interictal discharges can provide information about the precise source of these discharges (Laufs and Duncan, 2007). This technique may also be used to investigate the possibility that propagation of the seizure discharge to the contralateral hippocampus underlies the amnesic episodes of TEA (Hamandi et al., 2007).

\section{Prognosis}

The long-term prognosis of TEA for cognitive function is uncertain. The clinical impression gained from studying a small number of patients several years after the 
onset and treatment of their seizures is that memory function does not decline dramatically, at least once treatment is established. However, long-term follow up studies, with longitudinal neuropsychological testing and brain imaging, will be necessary to investigate prognosis in more detail.

\section{Syndrome classification}

The delineation of TEA as a distinct epilepsy syndrome requires further investigation of its relation to other forms of late-onset temporal lobe epilepsy (TLE). It will be important to establish, for example, what proportion of patients newly diagnosed with TLE in later life i) also experiences episodes of isolated amnesia upon direct questioning, ii) commonly experiences seizures upon waking, iii) has olfactory hallucinations and iv) typically has ictal and post-ictal dysfunction of a similar duration, 30 minutes and longer, to the amnesic attacks of TEA. Furthermore, the neuropsychological profile of such patients needs to be directly compared with that of TEA, including measures of long-term forgetting and remote memory.

\subsubsection{Interictal memory impairment in TEA}

The pervasive and unusual forms of interictal memory impairment associated with TEA may be important in the wider population of people with epilepsy (Butler and Zeman, 2008b). As discussed in Chapter 5, the investigation of ALF and RMI is not straightforward, especially in the presence of other factors known to impair memory, such as gross structural brain pathology, anticonvulsant polytherapy and psychosocial disadvantage. Nevertheless, the standardisation of experimental designs and careful selection of control populations should permit the investigation of these phenomena in a broader clinical setting. Besides their clinical relevance, ALF and RMI raise a number of important theoretical questions.

\section{Accelerated long-term forgetting}

The phenomenon of ALF demonstrates that, even when encoded successfully, new memories remain vulnerable for an extended period. As discussed in Chapter 1, memory consolidation is widely held to proceed in two stages: early, or 'cellular' 
consolidation, which involves protein synthesis and synaptic modification in the medial temporal lobes, and late, or 'systems', consolidation, which requires interaction between the medial temporal lobes and other cortical regions and the establishment of memory traces independent of the hippocampus. ALF, interpreted as a disturbance of systems consolidation, is compatible with this theoretical distinction. In humans, the existence of long-term consolidation processes has previously been inferred from patients with retrograde amnesia following brain damage. It has therefore been difficult to explore the underlying psychological and neural mechanisms, which remain unclear. Patients exhibiting ALF offer a unique opportunity to investigate human memory consolidation under well-controlled experimental conditions.

The pathophysiological basis of ALF, and in particular its relation to brain pathology and seizure discharges, remains unclear. Further analysis of the MRI data acquired in this study, including manual tracing of the parahippocampal cortices (Pruessner et al., 2002) and automated anatomical brain segmentation (Hammers et al., 2003; Heckemann et al., 2006), is already underway to examine areas beyond the hippocampus. In addition, alternative neuroimaging techniques, such as T2 relaxometry, spectroscopy, diffusion tensor imaging and functional MRI, may reveal correlated regional abnormalities that were not apparent in this study. If ALF is, in fact, a mild form of the amnesic syndrome, then it may be detectable in other populations, such as people with early Alzheimer's disease or mild cognitive impairment. If, instead, it is primarily a result of epileptic discharges in the temporal lobes, then correlations might be observed between EEG measures and the severity of forgetting. Furthermore, a reduction in ALF may be predicted to occur upon treatment with anticonvulsant medication, perhaps even once overt seizures have been controlled. The effect of subclinical epileptiform discharges on cognitive function is, however, a controversial topic (Aldenkamp and Arends, 2004; Binnie, 2003).

Since non-declarative memory systems are thought to be independent of the medial temporal lobes (Squire and Zola-Morgen, 1996), it is expected that ALF will not affect procedural memory or perceptual priming. However, this hypothesis is yet to be explored. 
The frequent occurrence of amnesic attacks upon waking is particularly intriguing given the hypothesised role of sleep in memory consolidation (Stickgold, 2005). The work reported in this thesis demonstrates that, in TEA, excessive forgetting occurs between 30 minutes and one week. More precise investigation of the time course of forgetting, together with extended EEG monitoring of TEA patients with frequent interictal discharges, will be important in elucidating the relationship between ALF and sleep.

ALF also raises questions for cognitive neuropsychology. The phenomenon may be primarily a deficit at the stage of memory encoding, detectable with more sensitive measures than those employed in this study. It has been suggested that deficits in the binding of distinct perceptual features of a stimulus, such as its shape and colour, may lead to impairments in long-term relational memory in older people (Chalfonte and Johnson, 1996). Alternatively, the problem may be one of memory storage: patients with ALF may be overly susceptible to retroactive interference from cognitive activity interposed between learning and memory retrieval (Cowan et al., 2004; Della Sala et al., 2005). Finally, ALF might reflect an inability to use contextual cues to assist in memory retrieval, particularly when the retrieval context is different from that at encoding (Becker et al., 1993; Smith et al., 1978).

\section{Remote memory impairment}

The temporally extensive but patchy autobiographical amnesia associated with TEA is not easily explained by current theoretical models of memory (Butler et al., 2007; Manes et al., 2001). If the pathophysiological processes underlying TEA are localised within the medial temporal lobes, standard consolidation theory would predict a relatively steep gradient of retrograde memory impairment. Multiple trace theory, on the other hand, might be able to account more naturally for the extensiveness of the deficit but would still have difficulty explaining its patchy nature and the impression that salient, often rehearsed memories seem to be most prominently affected. It has recently been shown that autobiographical memory networks, involving not only medial temporal structures, but also medial parietal and frontal cortex, temporal polar regions and the temporoparietal junction, are functionally disrupted in patients with TLE (Addis et al., 2007). It is possible that the striking autobiographical memory 
deficits seen in TEA are similarly caused by widespread network disturbance rather than focal pathology.

Further work is needed to investigate the unusual pattern of impairment seen in TEA. Longitudinal assessment may reveal progressive loss of patients' remote autobiographical memories. This finding would help to refute the suggestion (Kopelman, 2000) that, for decades prior to the first clinical manifestations of TEA, subclinical seizure activity may be disrupting the encoding and consolidation of autobiographical memories. Neuroimaging techniques such as those mentioned in the previous section will enable exploration of the possibility that network dysfunction in TEA extends beyond the hippocampal complex to regions implicated in the ultimate storage of memory traces.

The neuropsychological characterisation of remote memory impairment in TEA is incomplete. The Modified Autobiographical Memory Interview used in this study was designed to cover each decade of the subject's life yet not take too long to administer. The reported 'patchiness' of remote autobiographical memory loss in TEA patients may have led to an underestimation of the extent of the deficit. Future investigations may benefit from selecting a small number of patients with significant remote memory complaints and applying a comprehensive battery of sensitive (Levine et al., 2002) and perhaps tailor-made (Manes et al., 2001) autobiographical tests, alongside other measures to examine memory for public events, famous faces and places, and general knowledge. Such work would be very relevant to the ongoing debate about the neural substrates and dissociability of these forms of remote memory.

\section{Topographical memory}

Thirty-six percent of patients in this study reported new difficulties finding their way around previously familiar environments (Butler et al., 2007). The phenomenon was not explored further here and needs to be addressed in future work. The crucial role of the hippocampus in spatial memory is well established (Burgess et al., 2002; O'Keefe and Nadel, 1978), and spatial memory impairment has previously been demonstrated in TLE (Abrahams et al., 1997; Astur et al., 2002; Spiers et al., 2001). Several areas have received particular attention in the neuroscientific literature of human spatial 
memory. Firstly, the nature of the spatial framework supported varies across brain regions: the hippocampus appears to encode allocentric spatial representations, the posterior parietal cortex supports egocentric processing and retrosplenial regions may enable the interaction of the two (Burgess, 2008). Secondly, interest has also focussed upon the degree to which hippocampal involvement in spatial memory reflects a more general function in the processing of relational memory (Eichenbaum et al., 1999; Kumaran and Maguire, 2005). Thirdly, the temporal extent of remote spatial memory impairment in patients with medial temporal lobe damage has been a source of debate: very remote spatial memory does appear to be intact in patients with hippocampal damage (Teng and Squire, 1999), but the degree of preserved detail has been questioned (Rosenbaum et al., 2000). TEA offers a new and potentially informative arena for investigation of these issues: later in life, in the absence of gross structural pathology, intermittent, focal hippocampal dysfunction appears to disrupt topographical memory alongside remote autobiographical memory.

\subsection{Conclusions}

In this thesis, I have reported the results of by far the largest study to date of transient epileptic amnesia - a form of epilepsy that is characterised by recurrent episodes of isolated memory loss, and which often goes unrecognised by physicians. Nevertheless, it has many distinctive features that enable it to be reliably differentiated from other causes of transient amnesia. Among these, its recurrent nature, the brevity of attacks, the often-incomplete anterograde amnesia, the association with waking and the frequent co-occurrence of olfactory hallucinations are particularly helpful clues. Many patients have prominent complaints of interictal memory dysfunction. Whilst some show subtle deficits on standard neuropsychological tests of memory, performance is usually within the normal range. However, this study has demonstrated two relatively novel forms of memory problem amongst these patients - ALF and autobiographical memory loss - which help to explain their subjective impression of memory failures. These two phenomena may be present in the wider population of patients with epilepsy, amongst whom subjective memory difficulties are common. 
TEA is associated with subtle hippocampal atrophy. Furthermore, a few single cases have shown acute hippocampal oedema and hypermetabolism on neuroimaging. These findings add weight to the hypothesis, suggested by the clinical features, that TEA is due to focal medial temporal lobe seizures. The hippocampal atrophy correlates with performance on standard tests of anterograde memory, in keeping with traditional understanding of the function of this brain region. However, the neural substrates of ALF and autobiographical memory loss remain unclear. It is possible that they are caused, at least in part, by recurrent, possibly subclinical, seizure activity rather than overt structural damage.

Besides their clinical importance, these unusual deficits have the potential to inform memory theory. The autobiographical memory loss that accompanies TEA will provide insight into the nature of remote memory and the controversial phenomenon of focal retrograde amnesia. ALF will shed new light upon processes of long-term memory consolidation. TEA therefore offers a 'natural laboratory' (Snyder, 1997) in which to test and modify theoretical models of memory.

I have argued that TEA deserves to be considered a distinct syndrome of epilepsy. This proposal is driven both by the observed clinical consistency amongst cases and by the importance of increasing recognition of the condition by physicians. The boundaries of the syndrome, together with its aetiology, pathophysiology and prognosis require further exploration. Nevertheless, its central ground has been mapped and promises to yield substantial clinical and theoretical insights in the future. 


\section{References}

Abrahams S, Pickering A, Polkey CE, Morris RG. Spatial memory deficits in patients with unilateral damage to the right hippocampal formation. Neuropsychologia 1997; 35: $11-24$.

Acharya V, Acharya J, Luders H. Olfactory epileptic auras. Neurology 1998; 51: 5661.

Acosta-Cabronero J, Williams GB, Pereira JMS, Pengas G, Nestor PJ. The impact of skull-stripping and radio-frequency bias correction on grey-matter segmentation for voxel-based morphometry. NeuroImage 2008; 39: 1654-1665.

Addis DR, Moscovitch M, McAndrews MP. Consequences of hippocampal damage across the autobiographical memory network in left temporal lobe epilepsy. Brain. 2007; 130: 2327-42.

Aldenkamp AP, Arends J. Effects of epileptiform EEG discharges on cognitive function: is the concept of "transient cognitive impairment" still valid? Epilepsy Behav 2004; 5: S25-34.

Alessio A, Bonilha L, Rorden C, Kobayashi E, Min LL, Damasceno BP, et al. Memory and language impairments and their relationships to hippocampal and perirhinal cortex damage in patients with medial temporal lobe epilepsy. Epilepsy \& Behavior 2006; 8: 593-600.

Andy OJ, Jurko MF, Hughes JR. The amygdala in relation to olfaction. Confin Neurol 1975; 37: 215-22.

Arndt J, Passannante A, Hirshman E. The effect of midazolam on implicit and explicit memory in category exemplar production and category cued recall. Memory 2004; 12: $158-173$.

Ashburner J, Friston KJ. Voxel-based morphometry - the methods. Neuroimage 2000; 11: 805-21.

Ashburner J, Friston KJ. Unified segmentation. NeuroImage 2005; 26: 839-851.

Astur RS, Taylor LB, Mamelak AN, Philpott L, Sutherland RJ. Humans with hippocampus damage display severe spatial memory impairments in a virtual Morris water task. Behavioural Brain Research 2002; 132: 77-84. 
Avis HH, Carlton PL. Retrograde amnesia produced by hippocampal spreading depression. Science 1968; 161: 73-75.

Bancaud J, Brunet-Bourgin F, Chauvel P, Halgren E. Anatomical origin of deja vu and vivid 'memories' in human temporal lobe epilepsy. Brain 1994; 117: 71-90.

Banos JH, LaGory J, Sawrie S, Faught E, Knowlton R, Prasad A, et al. Self-report of cognitive abilities in temporal lobe epilepsy: cognitive, psychosocial, and emotional factors. Epilepsy \& Behavior 2004; 5: 575-579.

Barr WB, Goldberg E, Wasserstein J, Novelly RA. Retrograde amnesia following unilateral temporal lobectomy. Neuropsychologia 1990; 28: 243-55.

Bartolomei F, Barbeau E, Gavaret M, Guye M, McGonigal A, Regis J, et al. Cortical stimulation study of the role of rhinal cortex in deja vu and reminiscence of memories. Neurology 2004; 63: 858-864.

Bartsch T, Alfke K, Stingele R, Rohr A, Freitag-Wolf S, Jansen O, et al. Selective affection of hippocampal CA-1 neurons in patients with transient global amnesia without long-term sequelae. Brain 2006; 129: 2874-84.

Baxendale SA, Paesschen W, Thompson PJ, Connelly A, Duncan JS, Harkness WF, et al. The relationship between quantitative MRI and neuropsychological functioning in temporal lobe epilepsy. Epilepsia 1998; 39: 158-166.

Bayley PJ, Gold JJ, Hopkins RO, Squire LR. The neuroanatomy of remote memory. Neuron 2005; 46: 799-810.

Becker JT, Wess J, Hunkin NM, Parkin AJ. Use of temporal context information in Alzheimer's disease. Neuropsychologia 1993; 31: 137-43.

Bell BD. WMS-III Logical Memory performance after a two-week delay in temporal lobe epilepsy and control groups. J Clin Exp Neuropsychol 2006; 28: 1435-43.

Bell BD, Fine J, Dow C, Seidenberg M, Hermann BP. Temporal lobe epilepsy and the selective reminding test: The conventional 30-minute delay suffices. Psychological Assessment 2005; 17: 103-109.

Bell BD, Giovagnoli AR. Recent innovative studies of memory in temporal lobe epilepsy. Neuropsychol Rev 2007; 17: 455-476.

Bender MB. Single episode of confusion with amnesia. Bull NY Acad Med 1960; 36: 197-207.

Benson DF, Marsden CD, Meadows JC. The amnesic syndrome of posterior cerebral artery occlusion. Acta Neurol Scand 1974; 50: 133-45. 
Bergin PS, Thompson PJ, Baxendale SA, Fish DR, Shorvon SD. Remote memory in epilepsy. Epilepsia 2000; 41: 231-239.

Bergin PS, Thompson PJ, Fish DR, Shorvon SD. The effect of seizures on memory for recently learned material. Neurology 1995; 45: 236-240.

Bernasconi N, Bernasconi A, Caramanos Z, Antel SB, Andermann F, Arnold DL. Mesial temporal damage in temporal lobe epilepsy: a volumetric MRI study of the hippocampus, amygdala and parahippocampal region. Brain 2003; 126: 462-469.

Bernasconi N, Bernasconi A, Caramanos Z, Dubeau F, Richardson J, Andermann F, et al. Entorhinal cortex atrophy in epilepsy patients exhibiting normal hippocampal volumes. Neurology 2001; 56: 1335-1339.

Bernasconi N, Duchesne S, Janke A, Lerch J, Collins DL, Bernasconi A. Whole-brain voxel-based statistical analysis of gray matter and white matter in temporal lobe epilepsy. NeuroImage 2004; 23: 717-723.

Bernasconi N, Natsume J, Bernasconi A. Progression in temporal lobe epilepsy: Differential atrophy in mesial temporal structures. Neurology 2005; 65: 223-228.

Berrington WP, Liddell DW, Foulds GA. A re-evaluation of the fugue. J Ment Sci 1956; 102: 280-6.

Binnie CD. Cognitive impairment during epileptiform discharges: is it ever justifiable to treat the EEG? Lancet Neurol 2003; 2: 725-30.

Blake RV, Wroe SJ, Breen EK, McCarthy RA. Accelerated forgetting in patients with epilepsy: evidence for an impairment in memory consolidation. Brain 2000; 123: 472483.

Blumenfeld H, Taylor J. Why do Seizures Cause Loss of Consciousness? Neuroscientist 2003; 9: 301-310.

Bonilha L, Alessio A, Rorden C, Baylis G, Damasceno BP, Min L, et al. Extrahippocampal gray matter atrophy and memory impairment in patients with medial temporal lobe epilepsy. Human Brain Mapping 2007a; 28: 1376-1390.

Bonilha L, Rorden C, Appenzeller S, Carolina Coan A, Cendes F, Min Li L. Gray matter atrophy associated with duration of temporal lobe epilepsy. NeuroImage 2006; 32: 1070-1079.

Bonilha L, Rorden C, Castellano G, Pereira P, Rio PA, Cendes F, et al. Voxel-based morphometry reveals gray matter network atrophy in refractory medial temporal lobe epilepsy. Arch Neurol 2004; 61: 1379-1384. 
Bonilha L, Rorden C, Halford JJ, Eckert M, Appenzeller S, Cendes F, et al. Asymmetrical extra-hippocampal grey matter loss related to hippocampal atrophy in patients with medial temporal lobe epilepsy. J Neurol Neurosurg Psychiatry 2007b; 78: 286-294.

Bradley WG, Daroff RB, Fenichel G, Jankovic J. Neurology in Clinical Practice. Philadelphia: Butterworth-Heineman, 2008.

Braffman BH, Zimmerman RA, Trojanowski JQ, Gonatas NK, Hickey WF, Schlaepfer WW. Brain MR: pathologic correlation with gross and histopathology. 1. Lacunar infarction and Virchow-Robin spaces. Am. J. Roentgenol. 1988; 151: 551558.

Breteler MMB, van Swieten JC, Bots ML, Grobbee DE, Claus JJ, van den Hout JHW, et al. Cerebral white matter lesions, vascular risk factors, and cognitive function in a population-based study: The Rotterdam Study. Neurology 1994; 44: 1246-52.

Bronen RA, Fulbright RK, Kim JH, Spencer SS, Spencer DD, al-Rodhan NR. Regional distribution of MR findings in hippocampal sclerosis. AJNR Am J Neuroradiol 1995; 16: 1193-1200.

Brown AS. Transient global amnesia. Psychonomic bulletin \& review 1998; 5: 401427.

Brown AS. A review of the déjà vu experience. Psychological Bulletin 2003; 129: $394-413$.

Burgess N. Spatial cognition and the brain. Ann NY Acad Sci 2008; 1124: 77-97.

Burgess N, Maguire EA, O'Keefe J. The human hippocampus and spatial and episodic memory. Neuron 2002; 35: 625-641.

Burnham WH. Retroactive amnesia: illustrative cases and a tentative explanation. Am. J. Psychol 1903; 14: 382-396.

Busch RM, Lineweaver TT, Naugle RI, Kim KH, Gong Y, Tilelli CQ, et al. ApoE-4 is associated with reduced memory in long-standing intractable temporal lobe epilepsy. Neurology 2007; 68: 409-414.

Butler CR, Graham KS, Hodges JR, Kapur N, Wardlaw JM, Zeman AZ. The syndrome of transient epileptic amnesia. Ann Neurol 2007; 61: 587-598.

Butler CR, Zeman A. A case of transient epileptic amnesia with radiological localization. Nat Clin Prac Neurol 2008a; 4: 516-521. 
Butler CR, Zeman AZ. Recent insights into the impairment of memory in epilepsy: transient epileptic amnesia, accelerated long-term forgetting and remote memory impairment. Brain 2008b; 131: 2243-2263.

Butters N. Alcoholic Korsakoff's syndrome: Some unresolved issues concerning etiology, neuropathology, and cognitive deficits. Journal of Clinical and Experimental Neuropsychology 1985; 7: 181-210.

Caine D, Watson JDG. Neuropsychological and neuropathological sequelae of cerebral anoxia: A critical review. Journal of the International Neuropsychological Society 2000; 6: 86-99.

Calabrese P, Markowitsch HJ, Durwen HF, Widlitzek H, Haupts M, Holinka B, et al. Right temporofrontal cortex as critical locus for the ecphory of old episodic memories. J Neurol Neurosurg Psychiatry 1996; 61: 304-10.

Cantor FC. Transient global amnesia and temporal lobe seizures. AAN presentation, 1971.

Cammalleri R, Gangitano M, D'Amelio M, Raieli V, Raimondo D, Camarda R. Transient topographical amnesia and cingulate cortex damage: a case report. Neuropsychologia 1996; 34: 321-326.

Caplan L. Transient global amnesia and migraine. Neurology 1981; 31: 1167-1170.

Carne RP, O'Brien TJ, Kilpatrick CJ, MacGregor LR, Hicks RJ, Murphy MA, et al. MRI-negative PET-positive temporal lobe epilepsy: a distinct surgically remediable syndrome. Brain 2004; 127: 2276-2285.

Cave CB, Squire LB. Intact verbal and nonverbal short-term memory following damage to the human hippocampus. Hippocampus 1992; 2: 151-163.

Cendes F, Andermann F, Gloor P, Evans A, Jones-Gotman M, Watson C, et al. MRI volumetric measurement of amygdala and hippocampus in temporal lobe epilepsy. Neurology 1993; 43: 719-725.

Chalfonte BL, Johnson MK. Feature memory and binding in young and older adults. Memory \& Cognition 1996; 24: 403-416.

Chan D, Henley SM, Rossor MN, Warrington EK. Extensive and temporally ungraded retrograde amnesia in encephalitis associated with antibodies to voltagegated potassium channels. Archives of Neurology 2007; 64: 404-410.

Chen C, Shih Y-H, Yen D-J, Lirng J-F, Guo Y-C, Yu H-Y, et al. Olfactory auras in patients with temporal lobe epilepsy. Epilepsia 2003; 44: 257-260. 
Chu K, Kang D-W, Kim J-Y, Chang K-H, Lee SK. Diffusion-weighted magnetic resonance imaging in nonconvulsive status epilepticus. Arch Neurol 2001; 58: 993 998.

Chugani HT, Shewmon DA, Khanna S, Phelps ME. Interictal and postictal focal hypermetabolism on positron emission tomography. Pediatr Neurol 1993; 9: 10-15.

Chung PW, Seo DW, Kwon JC, Kim H, Na DL. Nonconvulsive status epilepticus presenting as a subacute progressive aphasia. Seizure 2002; 11: 449-454.

Clayton NS, Dickinson A. Episodic-like memory during cache recovery by scrub jays. Nature 1998; 395: 272-4.

Clayton NS, Griffiths DP, Emery NJ, Dickinson A. Elements of episodic-like memory in animals. Philosophical Transactions: Biological Sciences 2001; 356: 1483-1491.

Cloyd J, Hauser W, Towne A, Ramsay R, Mattson R, Gilliam F, et al. Epidemiological and medical aspects of epilepsy in the elderly. Epilepsy Research 2006; 68: 39-48.

Conway MA, Pleydell-Pearce CW. The construction of autobiographical memories in the self-memory system. Psychol Rev 2000; 107: 261-88.

Corcoran R, Thompson P. Memory failure in epilepsy: retrospective reports and prospective recordings. Seizure 1992; 1: 37-42.

Corridan BJ, Leung SN, Jenkins IH. A case of sleeping and forgetting. Lancet 2001; 357: 524 .

Coste S, Ryvlin P, Hermier M, Ostrowsky K, Adeleine P, Froment JC, et al. Temporopolar changes in temporal lobe epilepsy: A quantitative MRI-based study. Neurology 2002; 59: 855-861.

Costello A, Fletcher PC, Dolan RJ, Frith CD, Shallice T. The origins of forgetting in a case of isolated retrograde amnesia following a haemorrhage: Evidence from functional imaging. Neurocase 1998; 4: 437-446.

Cowan N, Beschin N, Della Sala S. Verbal recall in amnesiacs under conditions of diminished retroactive interference. Brain 2004; 127: 825-834.

Cox JE, Mathews VP, Santos CC, Elster AD. Seizure-induced transient hippocampal abnormalities on MR: correlation with positron emission tomography and electroencephalography. AJNR Am J Neuroradiol 1995; 16: 1736-1738.

Crespel A, Baldy-Moulinier M, Coubes P. The relationship between sleep and epilepsy in frontal and temporal lobe epilepsies: practical and physiopathologic considerations. Epilepsia 1998; 39: 150-157. 
Croft PB, Heathfield KWG, Swash M. Differential diagnosis of transient amnesia. British Medical Journal 1973: 593-596.

Cronel-Ohayon S, Zesiger P, Davidoff V, Boni A, Roulet E, Deonna T. Deficit in memory consolidation (abnormal forgetting rate) in childhood temporal lobe epilepsy. pre and postoperative long-term observation. Neuropediatrics 2006; 37: 317-324.

Crovitz HF. Frequency of episodic memories as a function of their age. Bulletin of the Psychonomic Society 1974; 4: 517-518.

Curran HV, Birch B. Differentiating the sedative, psychomotor and amnesic effects of benzodiazepines: a study with midazolam and the benzodiazepine antagonist, flumazenil. Psychopharmacology 1991; 103: 519-523.

D'Esposito M, Alexander MP, Fisher R, McGlinchey-Berroth R, O'Connor M. Recovery of memory and executive function following anterior communicating artery aneurysm rupture. J Int Neuropsychol Soc 1996; 2: 565-70.

Dasheiff RM, Hawley RJ, Cuenco N, Lastinger L, Vuilleumier P. Pure transient amnesia during nonconvulsive status epilepticus. Neurology 1997; 48: 1736-1737.

Davidson M, Dorris L, O'Regan M, Zuberi SM. Memory consolidation and accelerated forgetting in children with idiopathic generalized epilepsy. Epilepsy \& Behavior 2007; 11: 394-400.

Davis HP, Small SA, Stern Y, Mayeux R, Feldstein SN, Keller FR. Acquisition, recall, and forgetting of verbal information in long-term memory by young, middleaged, and elderly individuals. Cortex 2003; 39: 1063-91.

De Groot JC, De Leeuw FE, Oudkerk M, Van Gijn J, Hofman A, Jolles J, et al. Cerebral white matter lesions and cognitive function: The Rotterdam scan study. Annals of Neurology 2000; 47: 145-151.

De Renzi E, Lucchelli F. Dense retrograde amnesia, intact learning capability and abnormal forgetting rate: a consolidation deficit? Cortex 1993; 29: 449-466.

De Renzi E, Lucchelli F, Muggia S, Spinnler H. Persistent retrograde amnesia following a minor trauma. Cortex 1995; 31: 531-542.

DeGiorgio CM, Tomiyasu U, Gott PS, Treiman DM. Hippocampal pyramidal cell loss in human status epilepticus. Epilepsia 1992; 33: 23-27.

Deisenhammer E. Transient global amnesia as an epileptic manifestation. Journal of Neurology 1981; 225: 289-292. 
Della Sala S, Cowan N, Beschin N, Perini M. Just lying there, remembering: improving recall of prose in amnesic patients with mild cognitive impairment by minimising interference. Memory 2005; 13: 435-40.

Dewar MT, Cowan N, Sala SD. Forgetting Due to Retroactive Interference: A Fusion of Muller and Pilzecker's (1900) Early Insights into Everyday Forgetting and Recent Research on Anterograde Amnesia. Cortex 2007; 43: 616-634.

Diehl B, LaPresto E, Najm I, Raja S, Rona S, Babb T, et al. Neocortical temporal FDG-PET hypometabolism correlates with temporal lobe atrophy in hippocampal sclerosis associated with microscopic cortical dysplasia. Epilepsia 2003; 44: 559-564. Dodrill CB. The relationship of neuropsychological abilities to seizure factors and to surgery for epilepsy. Acta Neurol Scand Suppl 1992; 140: 106-10.

Dudai Y. The neurobiology of consolidations, or, how stable is the engram? Annual Review of Psychology 2004; 55: 51-86.

Dufouil C, de Kersaint-Gilly A, Besancon V, Levy C, Auffray E, Brunnereau L, et al. Longitudinal study of blood pressure and white matter hyperintensities: The EVA MRI Cohort. Neurology 2001; 56: 921-926.

Dugan TM, Nordgren RE, O'Leary P. Transient global amnesia associated with bradycardia and temporal lobe spikes. Cortex 1981; 17: 633-637.

Ebbinghaus H. Über das Gedächtnis: Untersuchungen zur experimentellen Psychologie: Duncker \& Humblot, 1885.

Ebner A, Kerder MS. Electrical stimulation of the olfactory and gustatory cortex. In: Luders HO and Noachtar S, editors. Epileptic seizures: pathophysiology and clinical semiology. Vol Churchill Livingston. New York, 2000: 237-42.

Eichenbaum H, Dudchenko P, Wood E, Shapiro M, Tanila H. The hippocampus, memory, and place cells: is it spatial memory or a memory space? Neuron 1999; 23: 209-226.

Elixhauser A, Leidy NK, Meador K, Means E, Willian MK. The relationship between memory performance, perceived cognitive function, and mood in patients with epilepsy. Epilepsy Research 1999; 37: 13-24.

Ellenbogen JM, Hulbert JC, Stickgold R, Dinges DF, Thompson-Schill SL. Interfering with theories of sleep and memory: sleep, declarative memory, and associative interference. Current Biology 2006; 16: 1290-1294. 
Engel J. A Proposed diagnostic scheme for people with epileptic seizures and with epilepsy: report of the ILAE task force on classification and terminology. Epilepsia 2001; 42: 796-803.

Engel J, Aicardi J, Pedley TA, Moshe S, Dichter MA. Epilepsy: A comprehensive textbook: Lippincott Williams \& Wilkins, 2007.

Engmann B, Reuter M. A case history of sudden memory dysfunction - caused by transient epileptic amnesia. Aktuelle Neurologie 2003; 30: 350-353.

Eritaia J, Wood SJ, Stuart GW, Bridle N, Dudgeon P, Maruff P, et al. An optimized method for estimating intracranial volume from magnetic resonance images. Magnetic Resonance in Medicine 2000; 44: 973-977.

Evans JH. Transient loss of memory, an organic mental syndrome. Brain 1966; 89: 539-48.

Evans JJ, Breen EK, Antoun N, Hodges JR. Focal retrograde amnesia for autobiographical events following cerebral vasculitis: A connectionist account. Neurocase 1996; 2: 1-12.

Evans JJ, Graham KS, Pratt KH, Hodges JR. The impact of disrupted cortico-cortico connectivity: a long-term follow-up of a case of focal retrograde amnesia. Cortex 2003; 39: 767-790.

Ewers M, Teipel SJ, Dietrich O, Schonberg SO, Jessen F, Heun R, et al. Multicenter assessment of reliability of cranial MRI. Neurobiology of Aging 2006; 27: 10511059.

Fazekas F, Chawluk JB, Alavi A, Hurtig HI, Zimmerman RA. MR signal abnormalities at $1.5 \mathrm{~T}$ in Alzheimer's dementia and normal aging. Am.J.Roentgenol. 1987; 149: 351-356.

Fazekas F, Kleinert R, Offenbacher H, Schmidt R, Kleinert G, Payer F, et al. Pathologic correlates of incidental MRI white matter signal hyperintensities. Neurology 1993; 43: 1683-9.

Fergusson SM, Rayport M, Gardner E, Kass W, Weiner H, Reiser MF. Similarities in mental content of psychotic states, spontaneous seizures, dreams, and responses to electrical brain stimulation in patients with temporal lobe epilepsy. Psychosom Med 1969; 31: 479-498.

Fink GR, Markowitsch HJ, Reinkemeier M, Bruckbauer T, Kessler J, Heiss WD. Cerebral representation of one's own past: neural networks involved in autobiographical memory. Journal of Neuroscience 1996; 16: 4275-4282. 
First MB, Spitzer RL, Gibbon M, Williams JBW. Structured Clinical Interview for DSM-IV Axis I Disorders - Research Version, Non-patient Edition (SCID-I/NP). New York: Biometrics Research, New York State Psychiatric Institute 2002.

Fisher CM, Adams RD. Transient global amnesia. Acta Neurologica Scandinavica 1964; 40: 7-82.

Fisher RS, Vickrey BG, Gibson P, Hermann B, Penovich P, Scherer A, et al. The impact of epilepsy from the patient's perspective I. Descriptions and subjective perceptions. Epilepsy Res. 2000; 41: 39-51.

Focke NK, Thompson PJ, Duncan JS. Correlation of cognitive functions with voxelbased morphometry in patients with hippocampal sclerosis. Epilepsy \& Behavior 2008; 12: 472-476.

Fong CY, Delgado-Escueta AV. Ictal PET in temporal lobe epilepsy. J Neurol Neurosurg Psychiatry 1999; 67: 409.

Freed DM, Corkin S, Cohen NJ. Forgetting in H.M.: a second look. Neuropsychologia 1987; 25: 461-71.

Fried I, Spencer DD, Spencer SS. The anatomy of epileptic auras: focal pathology and surgical outcome. J Neurosurg 1995; 83: 60-66.

Fujii T, Moscovitch M, Nadel L. Memory consolidation, retrograde amnesia, and the temporal lobe. In: Boller F and Grafman J, editors. Handbook of neuropsychology. Amsterdam: Elsevier, 2000: 223-250.

Fujii T, Yamadori A, Endo K, Suzuki K, Fukatsu R. Disproportionate retrograde amnesia in a patient with herpes simplex encephalitis. Cortex 1999; 35: 599-614.

Gaffan D. What is a memory system? Horel's critique revisited. Behav Brain Res 2001; 127: 5-11.

Gallassi R. Epileptic amnesic syndrome: an update and further considerations. Epilepsia 2006; 47 Suppl 2: 103-5.

Gallassi R, Morreale A, Di Sarro R. Epileptic amnesic syndrome: Further observations and considerations. Bollettino - Lega Italiana Contro L'Epilessia 1990a; Issue 70-71.

Gallassi R, Morreale A, Di Sarro R, Lugaresi E. Epileptic amnesic syndrome. Epilepsia 1992; 33 Suppl 6: S21-S25.

Gallassi R, Morreale A, Lorusso S, Pazzaglia P, Lugaresi E. Epilepsy presenting as memory disturbances. Epilepsia 1988a; 29: 624-629. 
Gallassi R, Morreale A, Lorusso S, Pazzaglia P, Lugaresi E. Epileptic transient amnesia. Italian Journal of Neurological Sciences 1988b; Suppl 9: 37-39.

Gallassi R, Morreale A, Markowitsch HJ. Transient global amnesia and epilepsy. Transient global amnesia and related disorders. Toronto: Hogrefe and Huber, 1990b.

Gallassi R, Pazzaglia P, Lorusso S, Morreale A. Neuropsychological findings in epileptic amnesic attacks. European Neurology 1986; 25: 299-303.

Gil-Nagel A, Risinger MW. Ictal semiology in hippocampal versus extrahippocampal temporal lobe epilepsy. Brain 1997; 120: 183-92.

Gilbert GJ. Transient global amnesia: manifestation of medial temporal lobe epilepsy. Clin Electroencephalogr 1978; 9: 147-52.

Gilbert JJ, Benson DF. Transient global amnesia: report of two cases with definite etiologies. J Nerv Ment Dis 1972; 154: 461-4.

Giovagnoli AR, Avanzini G. Learning and memory impairment in patients with temporal lobe epilepsy: relation to the presence, type, and location of brain lesion. Epilepsia 1999; 40: 904-911.

Giovagnoli AR, Casazza M, Avanzini G. Visual learning on a selective reminding procedure and delayed recall in patients with temporal lobe epilepsy. Epilepsia 1995; 36: 704-11.

Giovagnoli AR, Mascheroni S, Avanzini G. Self-reporting of everyday memory in patients with epilepsy: relation to neuropsychological, clinical, pathological and treatment factors. Epilepsy Res 1997; 28: 119-28.

Gleissner U, Helmstaedter C, Elger CE. Right hippocampal contribution to visual memory: a presurgical and postsurgical study in patients with temporal lobe epilepsy. J Neurol Neurosurg Psychiatry 1998; 65: 665-669.

Gloor P, Olivier A, Quesney LF, Andermann F, Horowitz S. The role of the limbic system in experiential phenomena of temporal lobe epilepsy. Annals of Neurology 1982; 12: 129-144.

Gloor P, Salanova V, Olivier A, Quesney LF. The human dorsal hippocampal commissure: An anatomically identifiable and functional pathway. Brain 1993; 116 : 1249-1273.

Goddard E. General household survey, 2005: smoking and drinking among adults. London: Office for National Statistics 2006.

Godlewski S. Amnesic episodes (transient global amnesia).(Clinical study based on 33 unpublished cases). Sem Hop 1968; 44: 553-77. 
Goncalves Pereira PM, Insausti R, Artacho-Perula E, Salmenpera T, Kalviainen R, Pitkanen A. MR volumetric analysis of the piriform cortex and cortical amygdala in drug-refractory temporal lobe epilepsy. AJNR Am J Neuroradiol 2005; 26: 319-32.

Graff-Radford NR, Tranel D, Van Hoesen GW, Brandt JP. Diencephalic amnesia. Brain 1990; 113: 1-25.

Graham FK, Kendall BS. Memory-for-designs test: Revised general manual. Perceptual and Motor Skills 1968; 11: 218-220.

Graham KS, Hodges JR. Differentiating the roles of the hippocampal complex and the neocortex in long-term memory storage: evidence from the study of semantic dementia and Alzheimer's disease. Neuropsychology. 1997; 11: 77-89.

Greenberg DL, Rubin DC. The neuropsychology of autobiographical memory. Cortex 2003; 39: 687-728.

Guillery-Girard B, Desgranges B, Urban C, Piolino P, de la Sayette V, Eustache F. The dynamic time course of memory recovery in transient global amnesia. J Neurol Neurosurg Psychiatry 2004; 75: 1532-40.

Guyotat J, Courjon J. Les ictus amnésiques. J Med Lyon 1956; 37: 697-701.

Haas DC, Ross GS. Transient global amnesia triggered by mild head trauma. Brain 1986; 109: 251-257.

Halgren E, Wilson CL, Stapleton JM. Human medial temporal-lobe stimulation disrupts both formation and retrieval of recent memories. Brain \& Cognition 1985; 4: 287-295.

Hamandi K, Powell HWR, Laufs H, Symms MR, Barker GJ, Parker GJM, et al. Combined EEG-fMRI and tractography to visualise propagation of epileptic activity. J Neurol Neurosurg Psychiatry 2007; 79: 594-7 .

Hammers A, Allom R, Koepp MJ, Free SL, Myers R, Lemieux L, et al. Threedimensional maximum probability atlas of the human brain, with particular reference to the temporal lobe. Hum Brain Mapp 2003; 19: 224-47.

Harding A, Halliday G, Caine D, Kril J. Degeneration of anterior thalamic nuclei differentiates alcoholics with amnesia. Brain 2000; 123: 141-154.

Hauser WA, Annegers JF, Kurland LT. Incidence of epilepsy and unprovoked seizures in Rochester, Minnesota: 1935-1984. Epilepsia 1993; 34: 453-468.

Hawley J. Pure transient amnesia during nonconvulsive status epilepticus. Neurology 1997; 48: 1736-1737. 
Heckemann RA, Hajnal JV, Aljabar P, Rueckert D, Hammers A. Automatic anatomical brain MRI segmentation combining label propagation and decision fusion. Neuroimage 2006; 33: 115-26.

Helmstaedter C, Hauff M, Elger CE. Ecological validity of list-learning tests and selfreported memory in healthy individuals and those with temporal lobe epilepsy. J Clin Exp Neuropsych 1998; 20: 365-375.

Hendriks MPH, Aldenkamp AP, Alpherts WCJ, Ellis J, Vermeulen J, van der Vlugt H. Relationships between epilepsy-related factors and memory impairment. Acta Neurologica Scandinavica 2004; 110: 291-300.

Henry TR, Drury I, Brunberg JA, Pennell PB, P.E. M, Beydoun A. Focal cerebral magnetic resonance changes associated with partial status epilepticus. Epilepsia 1994; 35: $35-41$.

Hermann BP, Seidenberg M, Schoenfeld J, Davies K. Neuropsychological characteristics of the syndrome of mesial temporal lobe epilepsy. Archives of Neurology 1997; 54: 369-376.

Hodges JR. Transient amnesia. London WB Saunders, 1991.

Hodges JR, McCarthy RA. Autobiographical amnesia resulting from bilateral paramedian thalamic infarction. A case study in cognitive neurobiology. Brain 1993; 116: 921-940.

Hodges JR, Ward CD. Observations during transient global amnesia. A behavioural and neuropsychological study of five cases. Brain 1989; 112: 595-620.

Hodges JR, Warlow CP. Syndromes of transient amnesia: towards a classification. A study of 153 cases. Journal of Neurology, Neurosurgery \& Psychiatry 1990a; 53: 834843.

Hodges JR, Warlow CP. The aetiology of transient global amnesia. A case-control study of 114 cases with prospective follow-up. Brain 1990b; 113: 639-57.

Hughlings-Jackson J. On a particular variety of epilepsy (intellectual aura), one case with symptoms of organic brain disease. Brain 1888; 11 179-207.

Hughlings-Jackson J, Beevor CE. Case of tumour of the right temporo-sphenoidal lobe bearing on the localization of the sense of smell and on the interpretation of a particular variety of epilepsy. Brain 1890; 12: 346-57.

Hughlings-Jackson J, Colman WS. Case of epilepsy with tasting movements and "dreamy state": very small patch of softening in the left uncinate gyrus. Brain 1898; 21: $580-590$. 
Hughlings-Jackson J, Stewart JP. Epileptic attacks with a warning of a crude sensation of smell and with the intellectual aura (dreamy state) in a patient who had symptoms pointing to gross organic disease of the right temporo-sphenoidal lobe. Brain 1899; 22: 534-549.

Hunkin NM, Parkin AJ, Bradley VA, Burrows EH, Aldrich FK, Jansari A, et al. Focal retrograde amnesia following closed head injury: a case study and theoretical account. Neuropsychologia 1995; 33: 509-523.

Huppert FA, Kopelman MD. Rates of forgetting in normal ageing: a comparison with dementia. Neuropsychologia 1989; 27: 849-60.

Huppert FA, Piercy M. Dissociation between learning and remembering in organic amnesia. Nature 1978; 275: 317-8.

Huppert FA, Piercy M. Normal and abnormal forgetting in organic amnesia: effect of locus of lesion. Cortex 1979; 15: 385-90.

Inoue Y, Mihara T. Awareness and Responsiveness During Partial Seizures. Epilepsia 1998; 39: 7-10.

Inzitari D, Pantoni L, Lamassa M, Pallanti S, Pracucci G, Marini P. Emotional arousal and phobia in transient global amnesia. Arch Neurol 1997; 54: 866-73.

Isaac CL, Mayes AR. Rate of forgetting in amnesia: I. Recall and recognition of prose. Journal of Experimental Psychology: Learning, Memory, \& Cognition 1999a; 25: $942-962$.

Isaac CL, Mayes AR. Rate of forgetting in amnesia: II. Recall and recognition of word lists at different levels of organization. Journal of Experimental Psychology: Learning, Memory, \& Cognition 1999b; 25: 963-977.

Jacome DE. EEG features in transient global amnesia. Clin Electroencephalogr 1989; 20: 183-92.

Jansen JFA, Vlooswijk MCG, Majoie HJM, de Krom MC, Aldenkamp AP, Hofman PAM, et al. White matter lesions in patients with localization-related epilepsy. Investigative Radiology 2008; 43: 552.

Janz D. The grand mal epilepsies and the sleeping-waking cycle. Epilepsia 1962; 3: 69-109.

Jasper H, Rasmussen H. Studies of clinical and electrical responses to deep temporal stimulation in man with some consideration of functional anatomy. Res Publ Assoc Re Nerv Ment Dis 1958; 36: 316-334. 
Jokeit H, Daamen M, Zang H, Janszky J, Ebner A. Seizures accelerate forgetting in patients with left-sided temporal lobe epilepsy. Neurology 2001; 57: 125-126.

Jokeit H, Kramer G, Ebner A. Do antiepileptic drugs accelerate forgetting? Epilepsy Behav. 2005; 6: 430-432.

Jutila L, Ylinen A, Partanen K, Alafuzoff I, Mervaala E, Partanen J, et al. MR volumetry of the entorhinal, perirhinal, and temporopolar cortices in drug-refractory temporal lobe epilepsy. AJNR Am J Neuroradiol 2001; 22: 1490-1501.

Kalviainen R, Salmenpera T, Partanen K, Vainio P, Riekkinen PSP, Pitkanen A. Recurrent seizures may cause hippocampal damage in temporal lobe epilepsy. Neurology 1998; 50: 1377-1382.

Kanwisher N, McDermott J, Chun MM. The fusiform face area: a module in human extrastriate cortex specialized for face perception. J Neurosci 1997; 17: 4302-4311.

Kapur N. Transient epileptic amnesia: a clinically distinct form of neurological memory disorder. In: Markowitsch HJ, editor. Transient global amnesia and related disorders. New York: Hogrefe and Huber, 1990: 140-151.

Kapur N. Focal retrograde amnesia in neurological disease: a critical review. Cortex 1993a; 29: 217-234.

Kapur N. Transient epileptic amnesia - a clinical update and a reformulation. Journal of Neurology, Neurosurgery \& Psychiatry 1993b; 56: 1184-1190.

Kapur N. Focal retrograde amnesia and the attribution of causality: An exceptionally benign commentary. Cognitive Neuropsychology 2000; 17: 623-637.

Kapur N, Abbott P, Footitt D, Millar J. Long-term perceptual priming in transient global amnesia. Brain Cogn 1996a; 31: 63-74.

Kapur N, Ellison D, Smith MP, McLellan DL, Burrows EH. Focal retrograde amnesia following bilateral temporal lobe pathology. A neuropsychological and magnetic resonance study. Brain 1992; 115: 73-85.

Kapur N, Heath P, Meudell P, Kennedy P. Amnesia can facilitate memory performance: Evidence from a patient with dissociated retrograde amnesia. Neuropsychologia 1986; 24: 215-221.

Kapur N, Millar J, Abbott P, Carter M. Recovery of function processes in human amnesia: evidence from transient global amnesia. Neuropsychologia 1998; 36: 99107. 
Kapur N, Millar J, Colbourn C, Abbott P, Kennedy P, Docherty T. Very long-term amnesia in association with temporal lobe epilepsy: evidence for multiple-stage consolidation processes. Brain \& Cognition 1997; 35: 58-70.

Kapur N, Scholey K, Moore E, Barker S, Brice J, Thompson S. Long-term retention deficits in two cases of disproportionate retrograde amnesia. J Cog Neurosci 1996b; 8: 416-434.

Kapur N, Thompson P, Kartsounis LD, Abbott P. Retrograde amnesia: clinical and methodological caveats. Neuropsychologia 1999; 37: 27-30.

Kapur N, Young A, Bateman D, Kennedy P. Focal retrograde amnesia: a long term clinical and neuropsychological follow-up. Cortex 1989; 25: 387-402.

Keller SS, Mackay CE, Barrick TR, Wieshmann UC, Howard MA, Roberts N. Voxelbased morphometric comparison of hippocampal and extrahippocampal abnormalities in patients with left and right hippocampal atrophy. NeuroImage 2002a; 16: 23-31.

Keller SS, Roberts N. Voxel-based morphometry of temporal lobe epilepsy: An introduction and review of the literature. Epilepsia 2008; 49: 741-757.

Keller SS, Wieshmann UC, Mackay CE, Denby CE, Webb J, Roberts N. Voxel based morphometry of grey matter abnormalities in patients with medically intractable temporal lobe epilepsy: effects of side of seizure onset and epilepsy duration. J Neurol Neurosurg Psychiatry 2002b; 73: 648-655.

Kelley WM, Miezin FM, McDermott KB, Buckner RL, Raichle ME, Cohen NJ, et al. Hemispheric specialization in human dorsal frontal cortex and medial temporal lobe for verbal and nonverbal memory encoding. Neuron 1998; 20: 927-936.

Kilpatrick C, Murrie V, Cook M, Andrewes D, Desmond P, Hopper J. Degree of left hippocampal atrophy correlates with severity of neuropsychological deficits. Seizure 1997; 6: 213-8.

King D, Bronen RA, Spencer DD, Spencer SS. Topographic distribution of seizure onset and hippocampal atrophy: Relationship between MRI and depth EEG. Electroencephalography and Clinical Neurophysiology 1997; 103: 692-697.

Kongs SK, Thompson LL, Iverson GL, Heaton RK. Wisconsin Card Sorting Test - 64 Card Version (WCST-64) Professional Manual. Odessa Psychological Assessment Resources Inc., 2000.

Kopelman MD. Rates of forgetting in Alzheimer-type dementia and Korsakoff's syndrome. Neuropsychologia 1985; 23: 623-38. 
Kopelman MD. Focal retrograde amnesia and the attribution of causality: An exceptionally critical review. Cognitive Neuropsychology 2000; 17: 585-621.

Kopelman MD. Disorders of memory. Brain 2002a; 125: 2152-90.

Kopelman MD. Psychogenic amnesia. In: Baddeley AD, Kopelman MD and Wilson B, editors. The handbook of memory disorders. Chichester: John Wiley \& Sons Ltd, 2002b: 451-471.

Kopelman MD. Retrograde amnesia. In: Baddeley AD, Kopelman MD and Wilson BA, editors. The handbook of memory disorders. Chichester: John Wiley \& Sons Ltd, 2002c: $189-207$.

Kopelman MD, Panayiotopoulos CP, Lewis P. Transient epileptic amnesia differentiated from psychogenic "fugue": neuropsychological, EEG, and PET findings. Journal of Neurology, Neurosurgery \& Psychiatry 1994; 57: 1002-1004.

Kopelman MD, Stanhope N. Recall and recognition memory in patients with focal frontal, temporal lobe and diencephalic lesions. Neuropsychologia 1998; 36: 785-796. Kopelman MD, Wilson BA, Baddeley AD. The autobiographical memory interview: a new assessment of autobiographical and personal semantic memory in amnesic patients. J Clin Exp Neuropsychol 1989; 11: 724-44.

Kosmidisand M, Papanicolaou AC. Transient epileptic amnesia. In: Papanicolaou AC, editor. The amnesias: a clinical textbook of memory disorders. New York: Oxford University Press, 2006.

Kritchevsky M. Transient global amnesia and functional retrograde amnesia: contrasting examples of episodic memory loss. Philosophical Transactions of the Royal Society B: Biological Sciences 1997; 352: 1747-1754.

Kritchevsky M, Chang J, Squire LR. Functional amnesia: clinical description and neuropsychological profile of 10 cases. Learn Mem 2004; 11: 213-26.

Kroll NE, Markowitsch HJ, Knight RT, von Cramon DY. Retrieval of old memories: the temporofrontal hypothesis. Brain 1997; 120: 1377-99.

Krsek P, Mikuleck A, Druga R, Kubov H, Hlink Z, Suchomelov L, et al. Long-term behavioral and morphological consequences of nonconvulsive status epilepticus in rats. Epilepsy \& Behavior 2004; 5: 180-191.

Kumaran D, Maguire EA. The human hippocampus: cognitive maps or relational memory? J Neurosci 2005; 25: 7254-9. 
Kuzniecky RI, Burgard S, Bilir E, Morawetz R, Gilliam F, Faught E, et al. Qualitative MRI segmentation in mesial temporal sclerosis: clinical correlations. Epilepsia 1996; 37: 433-439.

Kwan P, Brodie MJ. Neuropsychological effects of epilepsy and antiepileptic drugs. Lancet 2001; 357: 216-222.

Lah S, Grayson S, Lee T, Miller L. Memory for the past after temporal lobectomy: impact of epilepsy and cognitive variables. Neuropsychologia 2004; 42: 1666-79.

Lah S, Lee T, Grayson S, Miller L. Effects of temporal lobe epilepsy on retrograde memory. Epilepsia 2006; 47: 615-625.

Lansberg MG, O'Brien MW, Norbash AM, Moseley ME, Morrell M, Albers GW. MRI abnormalities associated with partial status epilepticus. Neurology 1999; 52: 1021-7.

Laplane D, Truelle JL. The mechanism of transient global amnesia. Apropos of some unusual cases. Nouv Presse Med 1974; 3: 721-5.

Laufs Hab, Duncan JSab. Electroencephalography/functional MRI in human epilepsy: what it currently can and cannot do. Cur Op Neurol 2007; 20: 417-423.

Lazeyras F, Blanke O, Zimine I, Delavelle J, Perrig SH, Seeck M. MRI, 1H-MRS, and functional MRI during and after prolonged nonconvulsive seizure activity. Neurology 2000; 55: 1677-1682.

Lee AC, Bussey TJ, Murray EA, Saksida LM, Epstein RA, Kapur N, et al. Perceptual deficits in amnesia: challenging the medial temporal lobe 'mnemonic' view. Neuropsychologia 2005; 43: 1-11.

Lee BI, Lee BC, Hwang YM, Sohn YH, Jung JW, Park SC, et al. Prolonged ictal amnesia with transient focal abnormalities on magnetic resonance imaging. Epilepsia 1992; 33: 1042-1046.

Lencz T, McCarthy G, Bronen RA, Scott TM, Inserni JA, Sass KJ, et al. Quantitative magnetic resonance imaging in temporal lobe epilepsy: relationship to neuropathology and neuropsychological function. Ann Neurol 1992; 31: 629-37.

Lespinet V, Bresson C, N'Kaoua B, Rougier A, Claverie B. Effect of age of onset of temporal lobe epilepsy on the severity and the nature of preoperative memory deficits. Neuropsychologia 2002; 40: 1591-1600.

Levin HS, High WM, Jr., Eisenberg HM. Learning and forgetting during posttraumatic amnesia in head injured patients. J Neurol Neurosurg Psychiatry 1988; 51: 14-20. 
Levin HS, O'Donnell VM, Grossman RG. The Galveston Orientation and Amnesia Test. A practical scale to assess cognition after head injury. J Nerv Ment Dis 1979; 167: 675-84.

Levine B, Black SE, Cabeza R, Sinden M, McIntosh AR, Toth JP, et al. Episodic memory and the self in a case of isolated retrograde amnesia. Brain 1998; 121: 19511973.

Levine B, Svoboda E, Hay JF, Winocur G, Moscovitch M. Aging and autobiographical memory: dissociating episodic from semantic retrieval. Psychol Aging 2002; 17: 677-89.

Levine B, Turner GR, Tisserand D, Hevenor SJ, Graham SJ, McIntosh AR. The functional neuroanatomy of episodic and semantic autobiographical remembering: a prospective functional MRI study. J Cogn Neurosci 2004; 16: 1633-46.

Lewis P, Kopelman MD. Forgetting rates in neuropsychiatric disorders. Journal of Neurology, Neurosurgery \& Psychiatry 1998; 65: 890-898.

Lewis SL. Aetiology of transient global amnesia. Lancet 1998; 352: 397-399.

Li X, Breteler MMB, Bruyne MC, Meinardi H, Hauser WA, Hofman A. Vascular Determinants of Epilepsy: The Rotterdam Study. Epilepsia 1997; 38: 1216-1220.

Lieb JP, Engel J, Jr., Babb TL. Interhemispheric propagation time of human hippocampal seizures. I. Relationship to surgical outcome. Epilepsia 1986; 27: 28693.

Liu RSN, Lemieux L, Bell GS, Hammers A, Sisodiya SM, Bartlett PA, et al. Progressive neocortical damage in epilepsy. Ann Neurol 2003; 53: 312-324.

Lou H. Repeated episodes of transient global amnesia. Acta Neurologica Scandinavica 1968; 44: 612-618.

Lucchelli F, Spinnler H. Ephemeral new traces and evaporated remote engrams: a form of neocortical temporal lobe amnesia? A preliminary case report. Neurocase 1998; 4 447-459.

Lux S, Kurthen M, Helmstaedter C, Hartje W, Reuber M, Elger CE. The localizing value of ictal consciousness and its constituent functions. Brain 2002; 125: 26912698.

MacLullich AMJ, Wardlaw JM, Ferguson KJ, Starr JM, Seckl JR, Deary IJ. Enlarged perivascular spaces are associated with cognitive function in healthy elderly men. $J$ Neurol Neurosurg Psychiatry 2004; 75: 1519-1523. 
Maguire EA. Neuroimaging studies of autobiographical event memory. Philos Trans R Soc Lond B Biol Sci 2001; 356: 1441-51.

Maheu G, Adam C, Hazemann P, Baulac M, Samson S. A case of postictal transient anterograde and retrograde amnesia. Epilepsia 2004; 45: 1459-1460.

Malykhin NV, Bouchard TP, Ogilvie CJ, Coupland NJ, Seres P, Camicioli R. Threedimensional volumetric analysis and reconstruction of amygdala and hippocampal head, body and tail. Psychiatry Research: Neuroimaging 2007; 155: 155-165.

Mameniskiene R, Jatuzis D, Kaubrys G, Budrys V. The decay of memory between delayed and long-term recall in patients with temporal lobe epilepsy. Epilepsy Behav. 2006; 8: 278-288.

Manes F, Graham KS, Zeman A, de Lujan Calcagno M, Hodges JR. Autobiographical amnesia and accelerated forgetting in transient epileptic amnesia. J Neurol Neurosurg Psychiatry 2005; 76: 1387-91.

Manes F, Hodges JR, Graham KS, Zeman A. Focal autobiographical amnesia in association with transient epileptic amnesia. Brain 2001; 124: 499-509.

Manford M, Fish DR, Shorvon SD. An analysis of clinical seizure patterns and their localizing value in frontal and temporal lobe epilepsies. Brain 1996; 119: 17-40.

Manning L. Focal retrograde amnesia documented with matching anterograde and retrograde procedures. Neuropsychologia 2002; 40: 28-38.

Manning L, Chassagnon S, Hirsch E, Kehrli P, Maitrot D. Selective memory impairment for public events in a patient with left temporal lobe epilepsy. Epilepsy Research 2005; 64: 45-47.

Manning L, Voltzenlogel V, Chassagnon S, Hirsch E, Kehrli P, Maitrot D. Selective memory impairment for public events associated with accelerated forgetting in a patient with left temporal lobe epilepsy. Rev Neurol (Paris) 2006; 162: 222-228.

Maravita A, Spadoni M, Mazzucchi A, Parma M. A new case of retrograde amnesia with abnormal forgetting rate. Cortex 1995; 31: 653-667.

Markowitsch HJ. Functional neuroimaging correlates of functional amnesia. Memory 1999; 7: 561-584.

Markowitsch HJ. Psychogenic amnesia. Neuroimage 2003; 20: S132-8.

Markowitsch HJ, Calabrese P, Liess J, Haupts M, Durwen HF, Gehlen W. Retrograde amnesia after traumatic injury of the fronto-temporal cortex. Journal of Neurology, Neurosurgery \& Psychiatry 1993; 56: 988-992. 
Marnet D, Noudel R, Peruzzi P, Bazin A, Bernard MH, Scherpereel B, et al. Dilatation of Virchow-Robin perivascular spaces (types III cerebral lacunae): radioclinical correlations. Rev Neurol (Paris) 2007; 163: 561-71.

Martin RC, Loring DW, Meador KJ, Lee GP. Differential forgetting in patients with temporal lobe dysfunction. Arch Clin Neuropsychol 1988; 3: 351-8.

Martin RC, Loring DW, Meador KJ, Lee GP, Thrash N, Arena JG. Impaired longterm retention despite normal verbal learning in patients with temporal lobe dysfunction. Neuropsychology 1991; 5: 3-12.

Martin RC, Sawrie SM, Roth DL, Gilliam FG, Faught E, Morawetz RB, et al. Individual memory change after anterior temporal lobectomy: a base rate analysis using regression-based outcome methodology. Epilepsia 1998; 39: 1075-1082.

Mayes AR, Isaac CL, Holdstock JS, Cariga P, Gummer A, Roberts N. Long-term amnesia: a review and detailed illustrative case study. Cortex 2003; 39: 567-603.

Maylor EA. Aging and forgetting in prospective and retrospective memory tasks. Psychol Aging 1993; 8: 420-8.

McKee RD, Squire LR. Equivalent forgetting rates in long-term memory for diencephalic and medial temporal lobe amnesia. J Neurosci 1992; 12: 3765-72.

McKenna P, Warrington EK. Testing for nominal dysphasia. J Neurol Neurosurg Psychiatry 1980; 43: 781-788.

Meador KJ, Adams RJ, Flanigin HF. Transient global amnesia and meningioma. Neurology 1985; 35: 769-771.

Meldrum BS, Brierley JB. Prolonged epileptic seizures in primates. Ischemic cell change and its relation to ictal physiological events. Arch Neurol 1973; 28: 10-7.

Meltzer CC, Adelson PD, Brenner RP, Crumrine PK, Cott A, Schiff DP, et al. Planned ictal FDG PET imaging for localization of extratemporal epileptic foci. Epilepsia 2000; 41: 193-200.

Mendes MH. Transient epileptic amnesia: an under-diagnosed phenomenon? Three more cases. Seizure 2002; 11:238-242.

Meo R, Bilo L, Striano S, Ruosi P, Estraneo A, Nocerino C. Transient global amnesia of epileptic origin accompanied by fever. Seizure 1995; 4: 311-317.

Metcalf K. Models of confabulation: A critical review and a new framework. Cognitive Neuropsychology 2007; 24: 23-47. 
Miller JW, Yanagihara T, Petersen RC, Klass DW. Transient global amnesia and epilepsy. Electroencephalographic distinction. Archives of Neurology 1987; 44: 629633.

Miller LA, Caine D, Harding A, Thompson EJ, Large M, Watson JD. Right medial thalamic lesion causes isolated retrograde amnesia. Neuropsychologia 2001; 39: 1037-1046.

Morrell F. Memory loss as a Todd's paralysis. Epilepsia 1980; 21: 185.

Moscovitch M, Nadel L. Consolidation and the hippocampal complex revisited: in defense of the multiple-trace model. Curr Opin Neurobiol 1998; 8: 297-300.

Moscovitch M, Nadel L, Winocur G, Gilboa A, Rosenbaum RS. The cognitive neuroscience of remote episodic, semantic and spatial memory. Current Opinion in Neurobiology 2006; 16: 179-190.

Moscovitch M, Rosenbaum RS, Gilboa A, Addis DR, Westmacott R, Grady C, et al. Functional neuroanatomy of remote episodic, semantic and spatial memory: a unified account based on multiple trace theory. J Anat 2005; 207: 35-66.

Motamedi G, Meador K. Epilepsy and cognition. Epilepsy \& Behavior 2003; 4: 25 38.

Motamedi GK, Meador KJ. Antiepileptic drugs and memory. Epilepsy \& Behavior 2004; 5: 435-439.

Mueller SG, Laxer KD, Cashdollar N, Buckley S, Paul C, Weiner MW. Voxel-based optimized morphometry (VBM) of gray and white matter in temporal lobe epilepsy (TLE) with and without mesial temporal sclerosis. Epilepsia 2006; 47: 900-907.

Müller GE, Pilzecker A. Experimentelle Beiträge zur Lehre vom Gedächtniss. Zeitschrift für Psychologie 1900; 1: 1-300.

Nadel L, Moscovitch M. Memory consolidation, retrograde amnesia and the hippocampal complex. Curr Opin Neurobiol 1997; 7: 217-27.

Newberg AB, Alavi A. PET in seizure disorders. Radiol Clin North Am 2005; 43: 7992.

Ng SKC, Hauser WA, Brust JCM, Susser M. Hypertension and the risk of new-onset unprovoked seizures. Neurology 1993; 43: 425-8.

Nielsen JM. Memory and amnesia. Los Angeles: San Lucas Press, 1958.

Noulhiane M, Piolino P, Hasboun D, Clemenceau S, Baulac M, Samson S. Autobiographical memory after temporal lobe resection: neuropsychological and MRI volumetric findings. Brain 2007; 130: 3184-3199. 
Novelly RA, Augustine EA, Mattson RH, Glaser GH, Williamson PD, Spencer DD, et al. Selective memory improvement and impairment in temporal lobectomy for epilepsy. Ann Neurol 1984; 15: 64-67.

O'Connor M, Butters N, Miliotis P, Eslinger P, Cermak LS. The dissociation of anterograde and retrograde amnesia in a patient with herpes encephalitis. J Clin Exp Neuropsychol 1992; 14: 159-78.

O'Connor M, Morin M, Verfaellie M, Greenblatt D, Doherty R, Cahn G, et al. Performance of temporal lobectomy patients on tests of remote memory. J Int Neuropsychol Soc 1999; 5: 117.

O'Connor M, Sieggreen MA, Ahern G, Schomer D, Mesulam M. Accelerated forgetting in association with temporal lobe epilepsy and paraneoplastic encephalitis. Brain \& Cognition 1997; 35: 71-84.

O'Connor M, Verfaellie M. The amnesic syndrome: overview and subtypes. In: Baddeley AD, Kopelman MD and Wilson B, editors. The handbook of memory disorders. Chichester: John Wiley and Sons, 2002.

O'Keefe J, Nadel L. The Hippocampus as a Cognitive Map: Oxford University Press, USA, 1978.

Ogden JA. Visual object agnosia, prosopagnosia, achromatopsia, loss of visual imagery, and autobiographical amnesia following recovery from cortical blindness: case M.H. Neuropsychologia 1993; 31: 571-589.

Olafsson E, Ludvigsson P, Gudmundsson G, Hesdorffer D, Kjartansson O, Hauser WA. Incidence of unprovoked seizures and epilepsy in Iceland and assessment of the epilepsy syndrome classification: a prospective study. Lancet Neurology 2005; 4: 627-634.

Olesen J, Jorgensen MB. Leao's spreading depression in the hippocampus explains transient global amnesia. A hypothesis. Acta Neurologica Scandinavica 1986; 73: 219-220.

Olivarius BD, Jensen TS. Transient global amnesia in migraine. Headache 1979; 19: 335-8.

Osterreith P, Rey A. Le test de copie d'une figure complexe. Arch Psychol 1944; 30 205-220.

Oxbury JM, Oxbury SM. Neuropsychology, memory and hippocampal pathology. In: Reynolds EH and Trimble MR, editors. The bridge between neurology and psychiatry. New York: Churchill Livingstone Inc, 1989. 
Oyegbile TO, Dow C, Jones J, Bell B, Rutecki P, Sheth R, et al. The nature and course of neuropsychological morbidity in chronic temporal lobe epilepsy. Neurology 2004; 62: 1736-1742.

Palmini AL, Gloor P, Jones-Gotman M. Pure amnestic seizures in temporal lobe epilepsy. Definition, clinical symptomatology and functional anatomical considerations. Brain 1992; 115: 749-769.

Panayiotopoulos CP. A clinical guide to epilepsy syndromes and their treatment. Chipping Norton: Bladon Medical Publishing, 2002.

Pantoni L, Bertini E, Lamassa M, Pracucci G, Inzitari D. Clinical features, risk factors, and prognosis in transient global amnesia: a follow-up study. Eur J Neurol 2005; $12: 350-6$.

Pantoni L, Lamassa M, Inzitari D. Transient global amnesia: a review emphasizing pathogenic aspects. Acta Neurol Scand 2000; 102: 275-83.

Parkin AJ, Leng NRC. Neuropsychology of the Amnesic Syndrome: Psychology Press, 1993.

Pauli E, Hildebrandt M, Romstock J, Stefan H, Blumcke I. Deficient memory acquisition in temporal lobe epilepsy is predicted by hippocampal granule cell loss. Neurology 2006; 67: 1383-1389.

Penfield W, Jasper HH. Epilepsy and the Functional Anatomy of the Human Brain: Little, Brown, 1954.

Penfield W, Perot P. The brain's record of auditory and visual experience: a final summary and discussion. Brain 1963; 86: 595-696.

Pengas G, Pereira JMS, Williams GB, Nestor PJ. Comparative reliability of total intracranial volume estimation methods and the influence of atrophy in a longitudinal semantic dementia cohort. J Neuroimaging 2008; in press.

Petersen RC, Smith G, Kokmen E, Ivnik RJ, Tangalos EG. Memory function in normal aging. Neurology 1992; 42: 396-.

Piazzini A, Canevini MP, Maggiori G, Canger R. The perception of memory failures in patients with epilepsy. European Journal of Neurology 2001; 8: 613-620.

Poser CM, Ziegler DK. Temporary amnesia as a manifestation of cerebrovascular insufficiency. Trans Am Neurol Assoc 1960; 85: 221-3.

Pritchard PB, III, Holmstrom VL, Roitzsch JC, Giacinto J. Epileptic amnesic attacks: benefit from antiepileptic drugs. Neurology 1985; 35: 1188-1189. 
Pruessner JC, Kohler S, Crane J, Pruessner M, Lord C, Byrne A, et al. Volumetry of temporopolar, perirhinal, entorhinal and parahippocampal cortex from high-resolution MR images: considering the variability of the collateral sulcus. Cereb Cortex 2002; 12: $1342-53$.

Pruessner JC, Li LM, Serles W, Pruessner M, Collins DL, Kabani N, et al. Volumetry of hippocampus and amygdala with high-resolution MRI and three-dimensional analysis software: minimizing the discrepancies between laboratories. Cereb Cortex 2000; 10: 433-42.

Pyszora NM, Barker AF, Kopelman MD. Amnesia for criminal offences: a study of life sentence prisoners. Journal of Forensic Psychiatry and Psychology 2003; 14: 475490.

Quigg M, Bertram EH, Jackson T. Longitudinal distribution of hippocampal atrophy in mesial temporal lobe epilepsy. Epilepsy Research 1997; 27: 101-110.

Quinette P, Guillery B, Desgranges B, de la Sayette V, Viader F, Eustache F. Working memory and executive functions in transient global amnesia. Brain 2003; 126: $1917-34$.

Quinette P, Guillery-Girard B, Dayan J, de la Sayette V, Marquis S, Viader F, et al. What does transient global amnesia really mean? Review of the literature and thorough study of 142 cases. Brain 2006; 129: 1640-58.

Ramsay RE, Rowan AJ, Pryor FM. Special considerations in treating the elderly patient with epilepsy. Neurology 2004; 62: 24S-29.

Ratti M, Galimberti C, Manni R, Tantara A. Remote memory impairment in temporal-lobe epilepsy. Seizure 1992; 1: 11.

Rausch R, Babb TL. Hippocampal neuron loss and memory scores before and after temporal lobe surgery for epilepsy. Archives of Neurology 1993; 50: 812-817.

Reminger SL, Kaszniak AW, Labiner DM, Littrell LD, David BT, Ryan L, et al. Bilateral hippocampal volume predicts verbal memory function in temporal lobe epilepsy. Epilepsy \& Behavior 2004; 5: 687-695.

Rempel-Clower NL, Zola-Morgen S, Squire LR, Amaral DG. Three cases of enduring memory impairment after bilateral damage limited to the hippocampal formation. Journal of Neuroscience 1996; 16: 5233-5255.

Ribot T. Diseases of memory. New York: Appleton-Century-Crofts, 1881. 
Rosenbaum RS, McKinnon MC, Levine B, Moscovitch M. Visual imagery deficits, impaired strategic retrieval, or memory loss: disentangling the nature of an amnesic person's autobiographical memory deficit. Neuropsychologia 2004; 42: 1619-35.

Rosenbaum RS, Priselac S, Black SE, Gao F, Nadel L, Moscovitch M. Remote spatial memory in an amnesic person with extensive bilateral hippocampal lesions. Nat Neurosci 2000; 3: 1044-1048.

Rowan AJ. Epilepsy and the Elderly. Epilepsy Behav. 2000; 1: S12-S14.

Rowan AJ, Protass LM. Transient global amnesia: clinical and electroencephalographic findings in 10 cases. Neurology 1979; 29: 869-872.

Rowan AJ, Rosenbaum DH. Ictal amnesia and fugue states. Adv Neurol 1991; 55: 357-367.

Rubin DC, Greenberg DL. Visual memory-deficit amnesia: a distinct amnesic presentation and etiology. Proceedings of the National Academy of Sciences of the United States of America 1998; 95: 5413-5416.

Rubin DC, Schulkind MD. Distribution of important and word-cued autobiographical memories in 20-, 35-, and 70-year-old adults. Psychol Aging 1997; 12: 524-35.

Rubin DC, Wenzel AE. One hundred years of forgetting: a quantitative description of retention. Psychological review 1996; 103: 734-760.

Salmenpera T, Kalviainen R, Partanen K, Pitkanen A. Hippocampal and amygdaloid damage in partial epilepsy: A cross-sectional MRI study of 241 patients. Epilepsy Research 2001; 46: 69-82.

Salmenpera T, Kononen M, Roberts N, Vanninen R, Pitkanen A, Kalviainen R. Hippocampal damage in newly diagnosed focal epilepsy: A prospective MRI study. Neurology 2005; 64: 62-68.

Sammaritano M, Gigli GL, Gotman J. Interictal spiking during wakefulness and sleep and the localization of foci in temporal lobe epilepsy. Neurology 1991; 41: 290-7.

Sander JW, Hart YM, Johnson AL, Shorvon SD. National General Practice Study of Epilepsy: newly diagnosed epileptic seizures in a general population. Lancet 1990; 336: 1267-71.

Sander K, Sander D. New insights into transient global amnesia: recent imaging and clinical findings. Lancet Neurol. 2005; 4: 437-444.

Schacter DL, Kihlstrom JF. Functional amnesia. Handbook of neuropsychology 1989; 3: 209-231.

Schacter DL, Tulving E. Memory systems 1994: MIT Press Cambridge, Mass, 1994. 
Schmahmann JD. Vascular syndromes of the thalamus. Stroke 2003; 34: 2264-2278.

Schmidt M. Rey auditory verbal learning test: a handbook. Los Angeles Western Psychological Services, 1996.

Schmidt R, Fazekas F, Kleinert G, Offenbacher H, Gindl K, Payer F, et al. Magnetic resonance imaging signal hyperintensities in the deep and subcortical white matter. A comparative study between stroke patients and normal volunteers. Arch Neurol 1992; 49: 825-827.

Scoville WB, Milner B. Loss of recent memory after bilateral hippocampal lesions. Journal of Neurology, Neurosurgery \& Psychiatry 1957; 20: 11-21.

Ségonne F, Dale AM, Busa E, Glessner M, Salat D, Hahn HK, et al. A hybrid approach to the skull stripping problem in MRI. Neuroimage 2004; 22: 1060-1075.

Seidenberg M, Kelly KG, Parrish J, Geary E, Dow C, Rutecki P, et al. Ipsilateral and contralateral MRI volumetric abnormalities in chronic unilateral temporal lobe epilepsy and their clinical correlates. Epilepsia 2005; 46: 420-430.

Shimamura AP, Janowsky JS, Squire LR. Memory for the temporal order of events in patients with frontal lobe lesions and amnesic patients. Neuropsychologia 1990; 28: 803-13.

Shuping JR, Toole JF, Alexander E, Jr. Transient global amnesia due to glioma in the dominant hemisphere. Neurology 1980; 30: 88-90.

Simons JS, Spiers HJ. Prefrontal and medial temporal lobe interactions in long-term memory. Nat Rev Neurosci 2003; 4: 637-48.

Slamecka NJ, McElree B. Normal forgetting of verbal lists as a function of their degree of learning. Journal of Experimental Psychology: Learning, Memory, and Cognition 1983; 9: 384-97.

Sled JG, Zijdenbos AP, Evans AC. A nonparametric method for automatic correction of intensity nonuniformity in MRI data. Medical Imaging, IEEE Transactions on 1998; 17: 87-97.

Smith SM. Fast robust automated brain extraction. Human Brain Mapping 2002; 17: 143-155.

Smith SM, Glenberg A, Bjork RA. Environmental context and human memory. Memory \& Cognition 1978; 6: 342-353.

Snyder PJ. Epilepsy as a "Natural Laboratory" for the study of human memory. Brain and Cognition 1997; 35 1-4. 
Spanaki MV, Kopylev L, Liow K, DeCarli C, Fazilat S, Gaillard WD, et al. Relationship of seizure frequency to hippocampus volume and metabolism in temporal lobe epilepsy. Epilepsia 2000; 41: 1227-1229.

Spencer SS, WIlliamson PD, Spencer DD, Mattson RH. Human hippocampal seizure spread studied by depth and subdural recording: the hippocampal commissure. Epilepsia 1987; 28: 479-89.

Spiers HJ, Burgess N, Maguire EA, Baxendale SA, Hartley T, Thompson PJ, et al. Unilateral temporal lobectomy patients show lateralized topographical and episodic memory deficits in a virtual town. Brain 2001; 124: 2476-89.

Squire LR. Two forms of human amnesia: an analysis of forgetting. Journal of Neuroscience 1981; 1: 635-640.

Squire LR. Memory and the hippocampus: a synthesis from findings with rats, monkeys, and humans. Psychol Rev. 1992; 99: 195-231.

Squire LR, Alvarez P. Retrograde amnesia and memory consolidation: a neurobiological perspective. Current Opinion in Neurobiology 1995; 5: 169-177.

Squire LR, Cohen N, Nadel L. The medial temporal lobe and memory consolidation: a new hypothesis. In: Eingertner E and Parker E, editors. Memory consolidation: psychobiology of cognition. Hillsdale, NJ: Lawrence Erlbaum, 1984: 185-210.

Squire LR, Knowlton B, Musen G. The structure and organization of memory. Annual Review of Psychology 1993; 44: 453-495.

Squire LR, Stark CE, Clark RE. The medial temporal lobe. Annu Rev Neurosci 2004; 27: 279-306.

Squire LR, Zola-Morgen S. Structure and function of declarative and nondeclarative memory systems. Proceedings of the National Academy of Sciences of the United States of America 1996; 93: 13515-13522.

Stephen LJ, Brodie MJ. Epilepsy in elderly people. Lancet 2000; 355: 1441-1446.

Stickgold R. Sleep-dependent memory consolidation. Nature 2005; 437: 1272-8.

Stonnington CM, Tan G, Kloppel S, Chu C, Draganski B, Jack Jr CR, et al. Interpreting scan data acquired from multiple scanners: A study with Alzheimer's disease. NeuroImage 2008; 39: 1180-1185.

Stracciari A, Ciucci G, Bianchedi G, Rebucci GG. Epileptic transient amnesia. European Neurology 1990; 30: 176-179. 
Stracciari A, Ghidoni E, Guarino M, Poletti M, Pazzaglia P. Post-traumatic retrograde amnesia with selective impairment of autobiographical memory. Cortex 1994; 30: 459-468.

Sutula TPa, Hagen Ja, Pitkanen Ab. Do epileptic seizures damage the brain? Current Opinion in Neurology 2003; 16: 189-195.

Svoboda E, McKinnon MC, Levine B. The functional neuroanatomy of autobiographical memory: a meta-analysis. Neuropsychologia 2006; 44: 2189-208.

Tanaka Y, Miyazawa Y, Hashimoto R, Nakano I, Obayashi T. Postencephalitic focal retrograde amnesia after bilateral anterior temporal lobe damage. Neurology 1999; 53: 344-350.

Tasch E, Cendes F, Li LM, Dubeau F, Andermann F, Arnold DL. Neuroimaging evidence of progressive neuronal loss and dysfunction in temporal lobe epilepsy. 1999.

Tassinari CA, Ciarmatori C, Alesi C, Cardinaletti L, Salvi F, Rubboli G, et al. Transient global amnesia as a postictal state from recurrent partial seizures. Epilepsia 1991; 32: 882-885.

Tellez-Zenteno JF, Dhar R, Hernandez-Ronquillo L, Wiebe S. Long-term outcomes in epilepsy surgery: antiepileptic drugs, mortality, cognitive and psychosocial aspects. Brain 2007; 130: 334-345.

Teng E, Squire LR. Memory for places learned long ago is intact after hippocampal damage. Nature 1999; 400: 675-7.

Tharp BR. The electroencephalogram in transient global amnesia. Electroencephalogr Clin Neurophysiol 1969; 26: 96-9.

Theodore WH, Bhatia S, Hatta J, Fazilat S, DeCarli C, Bookheimer SY, et al. Hippocampal atrophy, epilepsy duration, and febrile seizures in patients with partial seizures. Neurology 1999; 52: 132-.

Thomas-Antérion C, Koenig O, Navez M, Laurent B. Midazolam effects on implicit and explicit memory processes in healthy subjects. Psychopharmacology 1999; 145: 139-143.

Thompson PJ, Corcoran R. Everyday memory failures in people with epilepsy. Epilepsia 1992; 33 Suppl 6: S18-S20.

Thompson SA, Graham KS, Patterson K, Sahakian BJ, Hodges JR. Is knowledge of famous people disproportionately impaired in patients with early and questionable Alzheimer's disease? Neuropsychology. 2002; 16: 344-358. 
Tranel D, Jones R. Knowing what and knowing when. J Clin Exp Neuropsych 2006; 28: 43-66.

Tulving E. Episodic and semantic memory. Organization of memory 1972: 381-403.

Tulving E. Elements of episodic memory: Oxford University Press New York, 1983.

Tulving E. Episodic memory: From mind to brain. Annual Review of Psychology 2002; 53: 1-25.

Uijl SG, Leijten FSS, Arends JBAM, Parra J, van Huffelen AC, Moons KGM. The added value of [18F]-fluoro-D-deoxyglucose positron emission tomography in screening for temporal lobe epilepsy surgery. Epilepsia 2007; 48: 2121-2129.

Underwood BJ. Speed of learning and amount retained: a consideration of methodology. Psychol Bull 1954; 51: 276-82.

Upton D, Corcoran R, Fowler A, Thompson P. Autobiographical memory in epilepsy. Seizure 1992; 1: 14.

Van der Werf YD, Witter MP, Uylings HBM, Jolles J. Neuropsychology of infarctions in the thalamus: a review. Neuropsychologia 2000; 38: 613-627.

Van Paesschen W. Ictal SPECT. Epilepsia 2004; 45: 35-40.

Van Paesschen W, Connelly A, King MD, Jackson GD, Duncan JS. The spectrum of hippocampal sclerosis: A quantitative magnetic resonance imaging study. Ann Neurol 1997; 41: 41-51.

Vargha-Khadem F, Gadian DG, Watkins KE, Connelly A, Van Paesschen W, Mishkin M. Differential Effects of Early Hippocampal Pathology on Episodic and Semantic Memory. Science 1997; 277: 376.

Verfaellie M, Cermak LS. Neuropsychological issues in amnesia. In: Mertinez $\mathrm{J}$ and Kesner R, editors. Learning and memory: a biological view San Diego: Academic Press, 1991: 467-497.

Vermathen P, Laxer KD, Schuff N, Matson GB, Weiner MW. Evidence of neuronal injury outside the medial temporal lobe in temporal lobe epilepsy: $\mathrm{N}$-acetylaspartate concentration reductions detected with multisection proton MR spectroscopic imaging - initial experience. Radiology 2002; 226: 195-203.

Vermeulen J, Aldenkamp AP, Alpherts WCJ. Memory complaints in epilepsy: correlations with cognitive performance and neuroticism. Epilepsy Research 1993; 15: $157-170$. 
Vincent A, Buckley C, Schott JM, Baker I, Dewar B-K, Detert N, et al. Potassium channel antibody-associated encephalopathy: a potentially immunotherapy-responsive form of limbic encephalitis. Brain 2004; 127: 701-712.

Viskontas IV, McAndrews MP, Moscovitch M. Remote episodic memory deficits in patients with unilateral temporal lobe epilepsy and excisions. Journal of Neuroscience 2000; 20: 5853-5857.

Viskontas IV, McAndrews MP, Moscovitch M. Memory for famous people in patients with unilateral temporal lobe epilepsy and excisions. Neuropsychology 2002; 16: $472-80$.

Volpe BT, Herscovitch P, Raichle ME. Positron emission tomography defines metabolic abnormality in medial temporal lobes of two patients with amnesia after rupture and repair of anterior communicating artery aneurysm. Neurology 1984; 34: 188.

Voltzenlogel V, Despres O, Vignal J-P, Kehrli P, Manning L. One-year postoperative autobiographical memory following unilateral temporal lobectomy for control of intractable epilepsy. Epilepsia 2007; 48: 605-608.

Voltzenlogel V, Despres O, Vignal JP, Steinhoff BJ, Kehrli P, Manning L. Remote memory in temporal lobe epilepsy. Epilepsia 2006; 47: 1329-36.

Vuilleumier P, Despland PA, Regli F. Failure to recall (but not to remember): pure transient amnesia during nonconvulsive status epilepticus. Neurology 1996; 46: 10361039.

Walker M, Cross H, Smith S, Young C, Aicardi J, Appleton R, et al. Nonconvulsive status epilepticus: Epilepsy Research Foundation Workshop Reports. Epileptic Disord 2005; $7:$ :253-296.

Walker MP. A refined model of sleep and the time course of memory formation. Behavioral and Brain Sciences 2005; 28: 51-64.

Walker MP, Stickgold R. Sleep, memory, and plasticity. Annu Rev Psychol 2006; 57: 139-166.

Warren Z. Overgeneral memory for public and autobiographical events in depression and schizophrenia. Cognitive Neuropsychiatry 2007; 12: 301-321.

Warren-Gash C, Zeman A. Deja vu. Prac Neurol 2003; 3: 106-109.

Warrington EK. The recognition memory test. Windsor NFER, 1984.

Warrington EK, Weiskrantz L. Further analysis of the prior learning effect in amnesic patients. Neuropsychologia 1978; 16: 169-77. 
Watson C, Jack CR, Jr., Cendes F. Volumetric magnetic resonance imaging. Clinical applications and contributions to the understanding of temporal lobe epilepsy. Arch Neurol 1997; 54: 1521-1531.

Wechsler D. Wechsler Memory Scale (3rd ed.) New York: The Psychological Corporation, 1997.

Wechsler D. Wechsler Abbreviated Scale of Intelligence. San Antonio: The Psychological Corporation, 1999.

Wheeler MA, Stuss DT, Tulving E. Toward a theory of episodic memory: the frontal lobes and autonoetic consciousness. Psychol Bull 1997; 121: 331-54.

Wilson BA, Cockburn J, Baddeley AD. The Rivermead Behavioural Memory Test: Thames Valley Test Company, 1991.

Winbeck K, Etgen T, von Einsiedel HG, Rottinger M, Sander D. DWI in transient global amnesia and TIA: proposal for an ischaemic origin of TGA. Journal of Neurology, Neurosurgery \& Psychiatry 2005; 76: 438-441.

Witter M, Wouterlood F. The parahippocampal region: organisation and role in cognitive function. Oxford: Oxford University Press, 2002.

Woermann FG, Free SL, Koepp MJ, Ashburner J, Duncan JS. Voxel-by-voxel comparison of automatically segmented cerebral gray matter - a rater-independent comparison of structural MRI in patients with epilepsy. NeuroImage 1999; 10: 373384.

Zeman AZJ, Boniface SJ, Hodges JR. Transient epileptic amnesia: A description of the clinical and neuropsychological features in 10 cases and a review of the literature. Journal of Neurology, Neurosurgery \& Psychiatry 1998; 64: 435-443.

Zola-Morgan S, Squire LR, Ramus SJ. Severity of memory impairment in monkeys as a function of locus and extent of damage within the medial temporal lobe memory system. Hippocampus 1994; 4: 483-495. 


\section{APPENDIX A}

\section{TEA patient history pro forma}

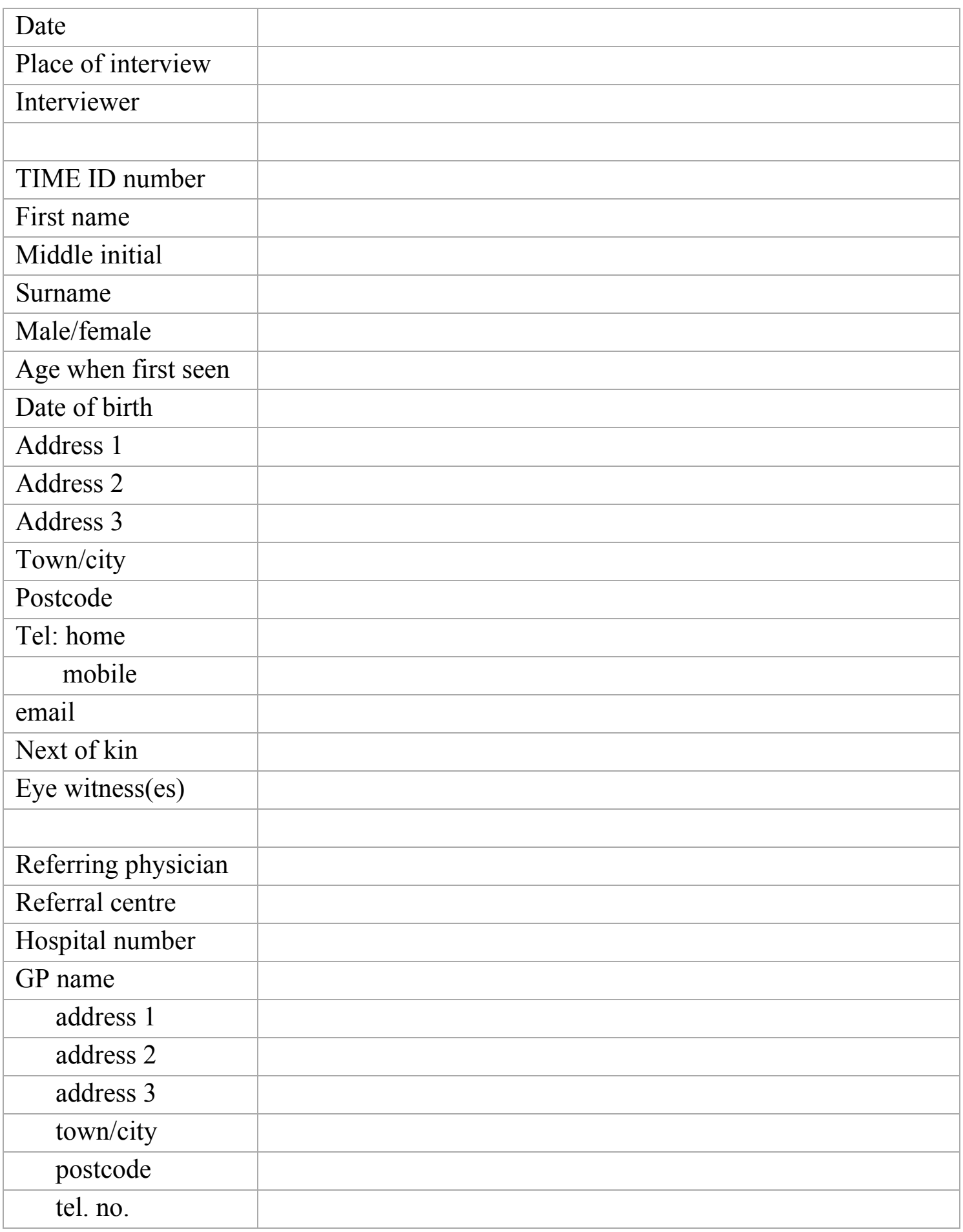




\begin{tabular}{|l|l|}
\hline Handedness & \\
\hline Place of Birth & \\
\hline Age left school & \\
\hline Further education & \\
\hline Total years of edn & \\
\hline Qualifications & \\
\hline Work history & \\
\hline
\end{tabular}

\section{History of presenting complaint}

First attack date

Last attack date

Frequency

Total number

Duration

Characteristic features (time of onset, triggers, aura, during attack (subjective), 'coming round', post-attack)

Extent of anterograde amnesia during attack

\section{Epilepsy Risk}

\section{Factors}

Birth Hx

Automatisms

Repetitive questioning

Extent of retrograde amnesia during attack 
Appendix A

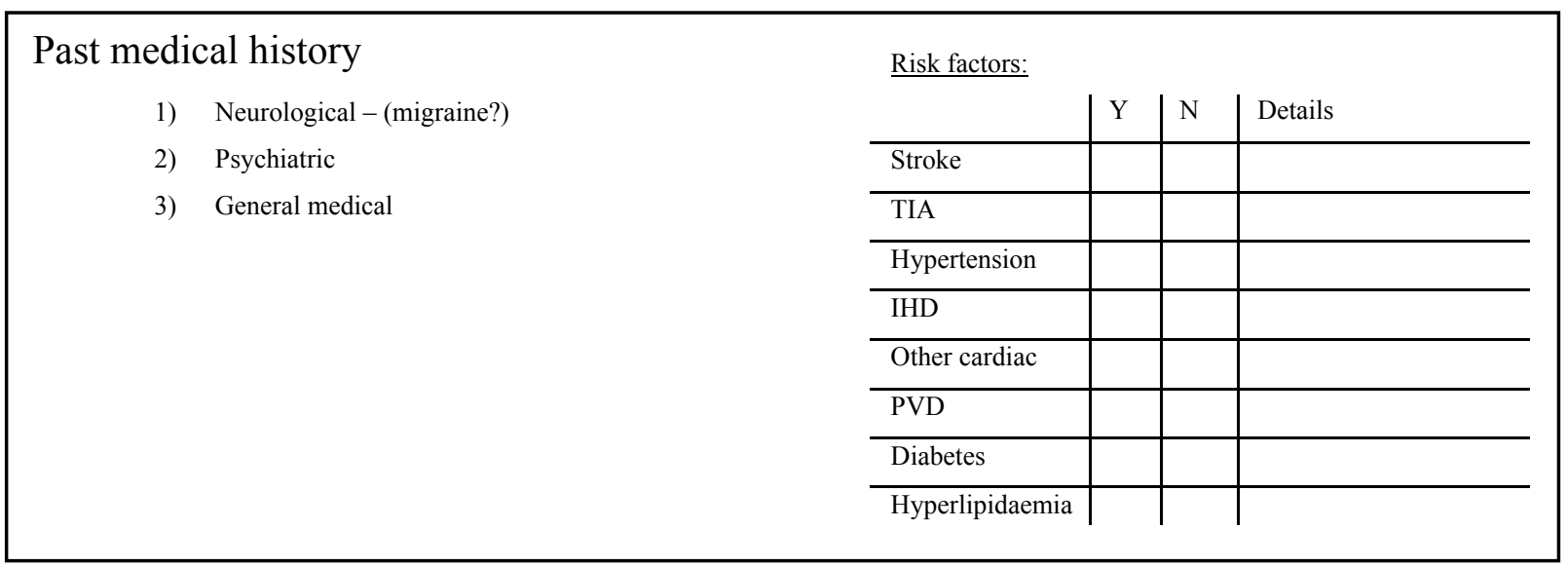

\section{Drug history}

1) anticonvulsants - current and past: dates, doses and frequency

Social history (inc. whether driving)

Family history

Systemic enquiry

Subjective memory assessment

1) Autobiographical events

i) anterograde (?AF)

ii) $\quad$ retrograde (?temporal gradient/patchy)

2) Public events - recent (Iraq war, 9/11, Rugby World cup)

- remote (Diana P of Wales, Falklands war, miners' strike, WWII) 
Appendix A 


\section{APPENDIX B}

\section{MRI scan visual assessment pro forma}

MRI ID number

Name of rater

Date of rating

\section{White Matter Hyperintensities (Fazekas et al. AJNR 1987;8:421-426)}

\begin{tabular}{|l|l|l|l|l|}
\hline Lesion & Score & Side & Comments & Rating Method \\
\hline Periventricular & & & & $0=$ absent \\
Hyperintensities & & & & $1=$ caps or pencil-thin lining around ventricles \\
(PVH) & & & & $2=$ smooth halo around ventricles \\
& & & $3=$ irregular PVH extending into the DWM \\
\hline Deep White & & & & $0=$ absent \\
Matter & & & & $1=$ punctate focal lesions only \\
Hyperintensities & & & & $2=$ beginning of confluence of foci \\
(DWMH) & & & & $3=$ large confluent areas \\
\hline
\end{tabular}

Enlarged Perivascular Spaces (MacClullich et al. JNNP 2004;75:1519-1523)

\begin{tabular}{|l|l|l|l|l|}
\hline Site & Score & Side & Comments & Rating Method \\
\hline Centrum Semi Ovale & & & & $0=$ absent \\
& & & & $1=$ mild (1-9 lesions) \\
& & & & \\
Basal Ganglia & & & & $3=$ moderate (10-19 lesions) \\
\hline Hippocampus & & & & \\
& & &
\end{tabular}

\section{Other Features}

\begin{tabular}{|l|l|}
\hline eg. focal atrophy, infarcts, \\
hippocampal \\
abnormalities, cortical \\
hyperintensity, basal \\
ganglia lesions, space- \\
occupying lesions
\end{tabular}


Appendix C 


\section{APPENDIX C}

\section{Illustrative Case Reports of Transient Epileptic Amnesia}

\section{Case A1 (ID number 32)}

TR is a 74-year old retired sales manager. He experienced 19 episodes of transient amnesia over a six-month period, each lasting between 15 and 30 minutes. They could occur at any time of day and were not preceded by any aura. On coming round, he would simply realise that he had no recollection of the last few minutes - "a bit like dozing off in front of the television and realising that you've missed the news". His wife's account is more detailed. She would notice her husband become "perplexed, anxious and slightly pale". He then began to ask questions indicating a loss of memory, such as "'What day is it? What am I doing here?", often in a repetitive manner. He would sometimes smack his lips, but was able to continue with cognitively demanding tasks.

One such attack happened whilst TR was driving towards home with his wife. He suddenly remarked "I don't know where I am? Where are we going?" and, when questioned, had no recollection of the day's events. He refused to let his wife take the wheel, but required instructions in order to follow the well-known route back to their house. His driving skills were unaffected and the memory loss resolved over about half an hour. A dramatic example of transient amnesia with otherwise preserved cognitive functioning occurred one evening at a bridge competition. TR and his wife are enthusiastic bridge players, competing several times a week. He was due to bid, but appeared confused and began to smack his lips. His wife said "It's your bid". He replied "Must I?" and then did so verbally, "two hearts", without using the required bidding cards. However, when reminded, he selected the correct bidding card and his wife raised him to game in four hearts, which he played and made without any apparent difficulty. The opponents did not notice anything untoward about TR, but later he remembered nothing of the episode.

In the course of investigating the amnesic spells, it was discovered that TR had intermittent complete heart block and a permanent pacemaker was inserted. This did not, however, alter the frequency of the attacks. A CT head revealed a small 
arachnoid cyst in the left temporal lobe. EEG was not performed. Treatment with Sodium Valproate abolished the attacks. However, TR has noticed ongoing memory difficulties of two types. Firstly, he finds that he forgets events more rapidly that before. This is particularly noticeable with his work for the local Conservative Party, of which he is the chairman. He frequently forgets conversations, having carried out certain administrative tasks, or meetings that he has attended. The second problem is a profound, but patchy loss of autobiographical memories for events of the past 40 to 50 years. This includes many episodes that occurred during four years spent living in Cologne and several business trips to North America and around Scandinavia. He is completely unable to remember his children's weddings, his brother's death or the many voyages he and his wife have made along the British canals on their narrow boat. He built the kitchen in their present house 11 years ago but does not remember doing so. These memories are not prompted by looking at photographs.

\section{Case A2 (ID number 124)}

FS is a 63-year old retired cleaner. She has very little recollection of the numerous amnestic attacks which have occurred over the past 12 years, except for the first one. She was sitting on the sofa in the living room, watching a workman install a new gas fire. The first symptom, as in the vast majority of her attacks, was a slight sense of nausea and "distance". She recalls that she then "suddenly didn't know who the man was". She remembers feeling puzzled but not frightened, and going through to the kitchen to ask her mother, who lived with her, what was happening. She recognised her mother and the house, but could not recall recent events. She lay down on the sofa and, although she has been told that she recovered soon thereafter, cannot recall anything further about that day. Some months prior to this episode, she had been knocked down by a car and sustained a head injury. She was not knocked out, did not sustain a skull fracture and did not experience any post-traumatic amnesia, but was quite shocked by the event. The first amnesic episode was, therefore, attributed both by her family and her general practitioner, to stress. A similar event occurred about 3 months later. This time a doctor told them it might have been caused by a "blood clot" and that, if the attacks recurred, she should lie down with her feet up and take an aspirin. This, then, is what she did for the next 12 years, at approximately monthly intervals, every time she felt the onset of an attack. The nature of the episodes 
remained the same, with unprovoked onset at any time of day, duration of about 20 minutes, and consisting of a retrograde amnesia for events of the recent past (occasionally up to a year or two) together with a complete or near-complete anterograde amnesia. Questioning was not typically repetitive and there were no associated automatisms, olfactory hallucinations or déjà vu. Other cognitive functions appeared entirely intact throughout.

A particularly dramatic episode occurred some 18 months after the death of FS's mother. Together with her daughter and sister, FS was at home sorting through a box of her mother's possessions. She went to make a cup of tea, returned and then said suddenly “Oh, I've such a sore stomach. What's in that box?" When her daughter explained, FS asked "Where's ma then?" She did not remember that her mother had died and was very distressed to hear it: "Where's she buried? Has she got a gravestone? Do we go and lay flowers?" The amnesia persisted for the usual 20 minutes before she said suddenly "Did I drink that tea?" and her memories returned. MRI brain was normal. Routine EEG revealed bitemporal epileptiform abnormalities. The attacks have ceased on Carbamazepine.

FS and her family also report ongoing difficulties with her memory. In particular, she rapidly forgets things that she has been told or that have happened to her. Last year, FS went to Southampton for a family wedding which was "great fun and very memorable". Upon returning, she gave her daughter a "blow-by-blow account" of all the amusing events that took place. However, one month later it was discovered that she had almost no recollection of the trip. Certain memories for autobiographical events prior to the onset of her seizures have also been affected. For example, FS is now unable to recall anything about a significant family holiday to Germany in 1975 , 18 years before her first amnestic attack, or about a cousin's wedding in 1980.

\section{Case A3 (ID number 95)}

EW is a 76-year old woman who experienced 6 amnestic attacks, the first of which occurred in 1980. She awoke in the night and said to her husband 'I'm frightened. I can't remember anything." They remained awake for some time during which it transpired that she had no recollection of recent events, including a children's birthday party they had hosted on the previous day. When she awoke in the morning, 
her memory had recovered and she was able to remember, if somewhat vaguely, the nocturnal attack. Approximately 18 months later there was another episode, the details of which are unclear as it was only witnessed in the later stages by her daughter. EW was gardening at home one afternoon. She does not remember what happened but was told afterwards that she had asked her daughter "How did those cabbages get there? I can't remember. Ask me some questions." Her daughter then asked her where her son was. She was able to remember that he was in Egypt, but not why he was there. She recovered quite rapidly.

There were no further episodes until September 1991. She rang her husband at work at $1 \mathrm{pm}$ one day and told him "I feel funny. I don't remember anything." She sounded distressed on the telephone and, when her husband arrived home shortly afterwards, she continued to question him repetitively about what was happening and complained of an occipital headache. She was taken to hospital with a suspected diagnosis of subarachnoid haemorrhage. However, her headache settled and her memory returned to normal over about six hours. She realised subsequently that her recollection of the day's events was rather vague. When she unpacked in hospital she congratulated her husband for having put all the right things in her bag. He then explained that she had packed it herself! He told her that she had joked with the ambulance men and she said that they must have thought she was a fraud. "Not true", he replied, "You repeated the same jokes over and over again!" She also described a "dream-like" memory of having gone out for coffee with a friend earlier in the morning. This was in fact true.

Another episode occurred one year later. Again, EW rang her husband at work in the middle of the day saying, "There are two strange men in the house and I don't know who they are." They were in fact two unemployed men whom she knew through a friend at church, and whom she had invited to do some gardening. After her husband's return, they noticed that a carriage clock and some silver ashtrays had gone missing. He telephoned for an ambulance and the police consecutively. It later emerged that EW had hidden them herself in the sideboard. She was back to normal by the evening and again had a "dream-like" recollection of the two gardeners arriving in the morning, but complete amnesia for subsequent events. A routine EEG was normal, but a sleep-deprived examination revealed epileptiform abnormalities over the left mid-temporal region. An MRI brain scan was unremarkable. 
Carbamazepine has abolished the attacks. There are no significant complaints of ongoing memory difficulties.

\section{Case A4 (ID number 83)}

GH is a 74-year old retired bank manager. The first amnesic episode occurred on his $72^{\text {nd }}$ birthday. He had been well during the day, helping his wife prepare for a family dinner that evening. At about 6pm, whilst watching television, he dozed off in his armchair. Upon waking 10 minutes later, he felt "a bit strange" and wandered into the kitchen. He remembers nothing more of that day's events. GH's wife confirms that, on waking from his nap, he entered the kitchen looking slightly dazed and, seeing all his children and grandchildren around, asked, "What are they all doing here? What's going on?" He did not remember that it was his birthday and repeatedly questioned his wife, apparently unable to take in new information. Mrs $\mathrm{H}$ insists that, apart from his memory loss, her husband was completely "with it", to the extent that, on the way to the GP's surgery, he "insisted on back-seat driving in his usual way". The amnesia persisted for several hours. GH believed that he was only 60 years old and was very distressed when, on asking about his family, he was told that his mother, father and sister had all died several years beforehand. By the next morning he was back to normal, but had no recollection of the events of the previous evening. A CT head, routine EEG and blood tests were all unremarkable and the attack was attributed to anxiety.

One month later, GH had another "memory blip". He describes waking in the morning feeling "a bit confused" and coming to about 15 minutes later, with only the vaguest recollection of what had just happened. He had no other symptoms. Again, his wife was able to give a much fuller description. At about $2 \mathrm{am}, \mathrm{GH}$ had sat up in bed, looking very anxious, and said "Oh, oh, the smell ... the taste". He did not respond to her and seemed to be having a nightmare. After a minute or so, he lay down and fell asleep again. At 7am, shortly after they had awoken, he came back from the bathroom saying, "I don't know what's going on. What's happening?" He was unable to recall recent events and facts, including that they were having their kitchen refurbished, but could remember all the names and dates of birth of their children and grandchildren. Mrs H says that, besides the memory loss, he was 
perfectly rational in conversation, and able to perform tasks such as dressing himself and making a cup of tea. There was nothing unusual in his appearance. After 15 minutes, the amnesia resolved.

There were about 18 further, stereotyped attacks over the next year and a half. The preceding brief, nocturnal panic-like episode was a consistent feature and was often associated with a vigorous swallowing automatism. The amnesia on wakening had a retrograde component extending back several years and a dense, but rarely complete anterograde component. During the episode, GH frequently complained of a strange, metallic taste or smell, a symptom that could also arise at other times. There were no clear triggers and the amnesia never lasted more than half an hour. A sleep-deprived EEG revealed bilateral epileptiform activity over the temporal lobes. MRI brain was normal. The attacks ceased on Carbamazepine. GH has no complaints of ongoing memory difficulties.

\section{Case A5 (ID number 55)}

SL is a 61-year old businessman who experienced at least seven amnestic attacks between February and August 2002. He has little recollection of his 'memory gaps' except for detecting an unusual smell beforehand. He would then come round, frequently in a different situation, feeling confused, disorientated and anxious because he didn't know what had happened. One early example occurred when he was alone in the car driving to a meeting in a nearby town. He says "I suddenly found myself on the borders of the neighboring county" whereupon he pulled over, extremely concerned, but recovered over about five minutes. The account from SL's wife is more revealing. She says that the first sign of an attack was that he would become quite "agitated", wringing his hands, biting his lip and grimacing. He then began to repeat the same questions or statements over and over again, often relating to the current date or his location, and did not seem to hold onto what was said to him. However, he was always responsive and appropriate, and could continue with whatever task he was currently engaged in. The episodes lasted between 30 and 60 minutes. One occurred whilst SL was eating with friends at Henley Royal Regatta. Despite the clear loss of memory for recent events, he was able to continue conversing and enjoying the races. He ordered the same bottle of wine five times 
during the 1 hour episode. There were no obvious triggers for the attacks and none occurred on waking. MRI brain and routine EEG were normal. The episodes ceased on Carbamazepine.

SL reports that since the onset of his amnestic attacks "tranches of my memory for the distant past have just disappeared". This loss appears to be patchy and extends back over 30 to 40 years. For example, he is unable to remember his first wedding, the first day at school of his eldest child, his father's funeral, a holiday on the French canals 20 years ago or a wedding in Madrid 3 years ago. In all of these cases, he knows that he used to be able to remember the events very well and, to some degree, is still able to recall the facts about them. However, he volunteers that he doesn't "have the visual picture", and cannot remember the "experience". Since beginning anticonvulsant therapy, his memory is better and he remembers clearly a holiday to Italy last year. $\mathrm{He}$ does not describe accelerated forgetting of newly learned material but complains of "appalling" daily difficulty with topographical memory - specifically for places that he hasn't visited recently.

\section{Case A6 (ID number 40)}

$\mathrm{DN}$ is a 68-year old man who recalls nothing of his amnesic attacks apart from a preceding "warm, tingly" feeling in both arms. His wife describes four episodes, the first of which occurred one morning in September 2000 whilst they were out shopping. DN suddenly remarked "I don't feel well" and became very pale and nauseated. He was unable to remember what they had been doing that morning, including their having taken the car to a garage for repairs. He repetitively questioned his wife about recent events. The episode lasted a number of hours and had completely resolved by the following day, although he had no recollection of it later. A diagnosis of transient global amnesia was made. At around the same time, he had been experiencing a number of migrainous headaches, associated with visual disturbance and tingling down his left side.

However, five months later he suffered a further prolonged episode of amnesia. He had been on a three-day course in York, which he greatly enjoyed and about which he told his wife in detail upon his return. The following morning, he awoke and asked his 
wife "Where am I?" and "What have I been doing?" He had no recollection of having attended the course, and repeatedly asked his wife the same questions. He got up and spent the day quietly at home. It is unclear exactly when he recovered, but the following day there was again a period at the dinner table during which he became pale and asked inappropriate questions. Certainly within a couple of days he had returned to normal, but his memory for the course never returned, even with the help of notes he had taken during it. Another amnesic episode occurred the day after making a speech at a local society of which he was president. He had felt fine and the speech had been enthusiastically received. He had not consumed excessive alcohol. He awoke the following morning completely unable to remember the events of the night before and, again, questioned his wife repetitively. He returned to normal over a few hours but the memory for the previous evening never returned.

A routine EEG showed slow wave abnormalities over the left temporal lobe. MRI scanning revealed a small $(1.5 \mathrm{~cm}$ diameter) meningioma underlying the right medial temporal lobe. Sodium Valproate abolished the transient amnestic attacks. However, DN complains of persistent anterograde and remote memory difficulties. He finds it difficult to learn new information and relies heavily upon his notebook. He says that he forgets events over a period of weeks including, for example, recent trips to visit his daughter. There has also been a pronounced loss of previously well-formed autobiographical memories from the past 15 to 20 years, long before the clinical onset of his epilepsy. This is particularly noticeable with regard to holidays, for example a visit to France last year and a long-planned trip to Italy upon his wife's retirement 6 years ago, neither of which he can remember at all. He is also unable to remember his daughter's wedding in France 11 years ago, which had previously been a frequent topic of reminiscence. There are, however, islands of preserved memory in between and his memory for more distant events is intact. Public event memory is less affected. He remembers the Falkland's war, the funeral of Diana Princess of Wales and the atrocities of September $11^{\text {th }} 2001$, although interestingly he does not recall being on holiday in Spain when they heard the news of it. DN also describes marked topographical memory difficulties, particularly with regard to picturing routes to places in his home town and finding his way around his daughters' houses. 


\section{APPENDIX D}

\section{Illustrative cases of recurrent transient amnesia excluded from the present study}

\section{Case B1}

A 62-year old retired secretary was witnessed to have three episodes of transient amnesia in 18 months. Each lasted between 30 minutes and two hours, and two occurred upon waking. There was retrograde amnesia for recent events, for example a visit of her grandchildren, accompanied by repetitive questioning and a vague recollection of the attack after its resolution. Following each attack, the patient felt "exhausted" and slept for several hours. There were no additional features suggestive of epilepsy, and no persistent memory difficulties. A routine EEG showed nonspecific slowing over the left temporal region but no frankly epileptiform features. Her neurologist diagnosed Transient Global Amnesia and prescribed no treatment. She has had no further recurrence over three years. Although the clinical features in this case are consistent with a diagnosis of Transient Epileptic Amnesia, it was not included in the series as it does not satisfy any of the three criteria used in the study for a diagnosis of epilepsy.

\section{Case B2}

An 80-year old widow described three episodes of transient amnesia, each lasting several hours. One occurred during a bridge competition, in which she participated successfully but was later unable to recall, and another on holiday in Tenerife, when she became disoriented and repeatedly questioned her companions. She gave a precise and detailed report of the episodes, but no witness account was available. She did not describe accelerated forgetting or remote autobiographical amnesia but reported problems with day-to-day, prospective memory. The patient had been diagnosed with temporal lobe epilepsy twenty years earlier after a series of attacks characterized by epigastric discomfort, anxiety and transient dysphasia. These resolved on Sodium Valproate, which she continued to take at a dose of 200mg three times daily. Routine EEG showed right temporal slowing, but no epileptiform features. She had a history 
of frequent migraine and current symptoms of major depression. Once again, these episodes may have been due to epilepsy. However, the case was not included as no eye-witness description of the episodes was available to confirm the occurrence of amnesia in the absence of other cognitive impairment during the attack.

\section{Case B3}

A 60-year old businessman first began to notice problems with his memory 10 years ago. Failure to remember tasks he had been assigned led to his demotion at work. He also noticed difficulties with route finding. Two years later he began to experience frequent, brief episodes of altered behavior and unresponsiveness of which he afterwards had no recollection. These lasted a few seconds, were characterized by repeated, stereotyped comments such as "yeah, that's right", and were occasionally preceded by a sense of déjà vu. He complained of gradually forgetting information over a period of days, as well as 'holes' in his autobiographical memory for the past decade. Standard memory testing was normal, as was a routine EEG and MRI brain scan. He was told that the problems were due to 'stress', until his general practitioner witnessed him have a complex partial seizure. Carbamazepine abolished the attacks, but his memory problems persisted. There were no witnessed episodes of pure, transient amnesia typical of TEA, so he was excluded from the study. The case, however, illustrates that complaints of accelerated forgetting and autobiographical amnesia can be associated with, and perhaps even predate, more typical temporal lobe seizures. 


\section{APPENDIX E}

\section{Everyday Memory Questionnaire (Thompson and Corcoran (1992))}

In the below questions, please circle your answer according to the following scheme:

$\mathbf{A}=$ not at all; $\mathbf{B}=$ about once in last 3 months $; \mathbf{C}=$ about once a month; $\mathbf{D}=$ about once

a week; $\mathbf{E}=$ about once a day; $\mathbf{F}=$ more than once a day

\section{How often do you:}

\begin{tabular}{|c|c|}
\hline 1) forget where you put something, eg losing something around the house? & A $B \quad C \quad D \quad E \quad F$ \\
\hline 2) fail to recognise places that you are told you have been to often before? & A $B \quad C \quad D \quad E \quad F$ \\
\hline $\begin{array}{l}\text { 3) fail to remember a change in your daily routine such as a change in the place } \\
\text { where something is kept or a change in the time something happens? }\end{array}$ & 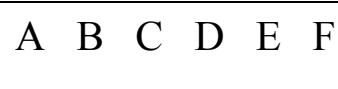 \\
\hline 4) have to go back to check whether you had done something you meant to do? & 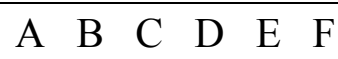 \\
\hline $\begin{array}{l}\text { 5) forget that you were told something yesterday, or a few days ago, and maybe } \\
\text { had to be reminded of it }\end{array}$ & A $\quad$ B $\quad$ C $\quad D \quad E$ F \\
\hline 6) let yourself ramble on to speak about unimportant or irrelevant things? & 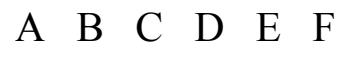 \\
\hline $\begin{array}{l}\text { 7) have difficulty picking up a new skill, eg finding it hard to learn a new game or } \\
\text { to work some gadget after you had practised it once or twice? }\end{array}$ & 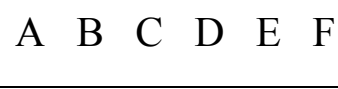 \\
\hline $\begin{array}{l}\text { 8) find that a word is 'on the tip of your tongue', you know what it is but cannot } \\
\text { find it? }\end{array}$ & 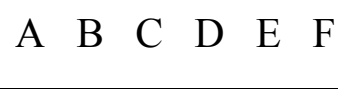 \\
\hline $\begin{array}{l}\text { 9) forget important details of what you did or forget what happened to you the } \\
\text { day before? }\end{array}$ & A $\quad$ B $\quad C \quad D \quad E \quad F$ \\
\hline 10) forget important details about yourself, eg date of birth, where you live? & 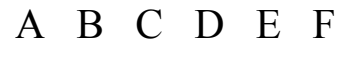 \\
\hline $\begin{array}{l}\text { 11) when talking to someone, forget what you had just said, maybe saying "what } \\
\text { was I talking about?" }\end{array}$ & 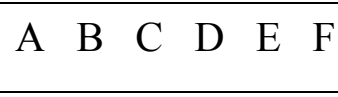 \\
\hline $\begin{array}{l}\text { 12) when reading a newspaper or magazine, being unable to follow the thread of } \\
\text { the story, lose track of what it was about? }\end{array}$ & 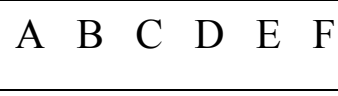 \\
\hline $\begin{array}{l}\text { 13) forget to tell somebody something important. Perhaps forget to pass on a } \\
\text { message or remind someone of something? }\end{array}$ & A $\quad$ B $\quad$ C $\quad D \quad E$ F \\
\hline 14) get the details of what somebody has told you mixed up or confused? & A $\quad$ B $\quad C \quad D \quad E \quad F$ \\
\hline 15) forget people's names? & 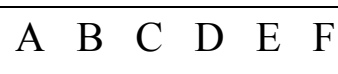 \\
\hline $\begin{array}{l}\text { 16) get lost or turn in the wrong direction on a journey, on a walk, or in a building } \\
\text { you have only been to once or twice before? }\end{array}$ & 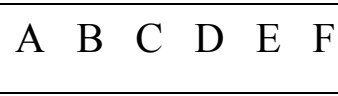 \\
\hline 17) repeat to someone what you have told them or ask the same question twice? & A $\quad$ B $\quad$ C $\quad D \quad E \quad F$ \\
\hline 18) fail to recognise by sight a close relative? & A $\mathrm{B} \quad \mathrm{C} \quad \mathrm{D} \quad \mathrm{E}$ \\
\hline
\end{tabular}


Appendix F 


\section{APPENDIX F}

\section{Very Long Term Memory Questionnaire (VTLMQ)}

1) Have you ever completely forgotten a holiday that you went on a few months or years ago?

Never Once Several Times Many Times $\quad$ Doesn't Apply

2) Have you ever completely forgotten that you went to a particular address a few months or years before?

Never Once Several Times Many Times Doesn't Apply

3) Have you ever completely forgotten that someone close to you died a few months or years before?

Never Once Several Times Many Times Doesn't Apply

4) Have you ever completely forgotten that someone close to you had hospital treatment a few months or years before?

Never Once Several Times Many Times Doesn't Apply

5) Have you ever completely forgotten being in hospital for something a few months or years before?

Never Once Several Times Many Times Doesn't Apply 
6) Have you ever completely forgotten that a close friend or relative was married?
Never OnCE
SEVERAL TIMES
MANY TIMES
DOESN'T APPLY

7) Have you ever completely forgotten that someone you know well had a baby?
NEVER
ONCE
SEveral Times
MANY TIMES
DOESN'T APPLY

8) Have you ever completely forgotten having attended a wedding a few months or years before?
NeVer ONCE
Several Times
MANY TIMES
DOESN'T APPLY

9) Have you ever completely forgotten having lived at a particular address a few months or years before?

Never Once Several Times Many Times $\quad$ Doesn't Apply

10) Have you ever met someone that you used to know very well a few months or years before, but treated them as a complete stranger?
Never OnCE
SEVERAL TIMES
MANY TIMES
DOESN'T APPLY

11) Have you ever completely forgotten being in a particular job a few months or years before?
NeVer
ONCE
SEVERAL Times
MANy TIMES
DOESN'T APPLY 
12) Have you ever completely forgotten doing a major piece of work or project a few months or years before?
NEVER ONCE
SEVERAL TIMES
MANY TIMES
DOESN'T APPLY

13) Have you ever completely forgotten the directions for how to get somewhere that was once very familiar to you several months or years before?

Never Once Several Times Many Times Doesn't Apply


Appendix F 


\section{APPENDIX G}

\section{Long-term forgetting task 1: Word list from Rey Auditory verbal learning task (RAVLT)}

Subject Name:

Date:

Start time: End time:

Criterion $=14 / 15$

\begin{tabular}{|c|c|c|c|c|c|c|c|c|c|c|c|c|c|c|c|c|c|c|}
\hline Word & \multicolumn{15}{|c|}{ Training } & \multirow[t]{2}{*}{ 30' } & \multirow[t]{2}{*}{1 wk } & \multirow[t]{2}{*}{$3 \mathrm{wk}$} \\
\hline & 1 & 2 & 3 & 4 & 5 & 6 & 7 & 8 & 9 & 10 & 11 & 12 & 13 & 14 & 15 & & & \\
\hline & & & & & & & & & & & & & & & & & & \\
\hline \multicolumn{19}{|l|}{ DRUM } \\
\hline \multicolumn{19}{|l|}{ CURTAIN } \\
\hline \multicolumn{19}{|l|}{ BELL } \\
\hline \multicolumn{19}{|l|}{ COFFEE } \\
\hline \multicolumn{19}{|l|}{ SCHOOL } \\
\hline \multicolumn{19}{|l|}{ PARENT } \\
\hline \multicolumn{19}{|l|}{$\begin{array}{l}\text { MOON } \\
\end{array}$} \\
\hline \multicolumn{19}{|l|}{ GARDEN } \\
\hline \multicolumn{19}{|l|}{$\begin{array}{ll}\text { HAT } \\
\end{array}$} \\
\hline \multicolumn{19}{|l|}{ FARMER } \\
\hline \multicolumn{19}{|l|}{ NOSE } \\
\hline \multicolumn{19}{|l|}{ TURKEY } \\
\hline \multicolumn{19}{|l|}{ COLOUR } \\
\hline \multicolumn{19}{|l|}{ HOUSE } \\
\hline \multicolumn{19}{|l|}{ RIVER } \\
\hline Total & & & & & & & & & & & & & & & & & & \\
\hline
\end{tabular}




\section{RAVLT Delayed Recognition Trial for Form A}

Instructions: The subject must respond Yes/No to each word that is read out according to whether they think the word was on the original list.

\begin{tabular}{|l|l|l|}
\hline \multicolumn{3}{|c|}{ Answers } \\
\hline BELL & MOON & TURKEY \\
\hline WINDOW (sa) & DOOR (pa) & TEACHER (sa) \\
\hline HAT & COFFEE & GARDEN \\
\hline BARN & RIVER & TREE \\
\hline HOME (sa) & HOT (pa) & PARENT \\
\hline NOSE & CURTAIN & DRUM \\
\hline SCHOOL & MOUSE (pa) & TOFFEE (pa) \\
\hline BALLOON (pa) & COLOUR & HOUSE \\
\hline ROSE (pa) & WATER (sa) & FARMER \\
\hline CHILDREN & HAND & BICYCLE \\
\hline
\end{tabular}

$\underline{\text { Key: }}$

List A word $=$ BOLD

Word with a semantic association to a word on list $\mathrm{a}=\mathrm{sa}$

Word phonemically similar to word on list $\mathrm{a}=\mathrm{pa}$ 


\section{Long-term forgetting task 2: Memory for Designs (MFD)}

$\begin{array}{lll}\text { MFD-A EXTENDED RECALL } & \text { Name: } & \text { Date: } \\ \text { Criterion }=19 / 21(90 \%) & \text { Start time : } & \text { End time }\end{array}$

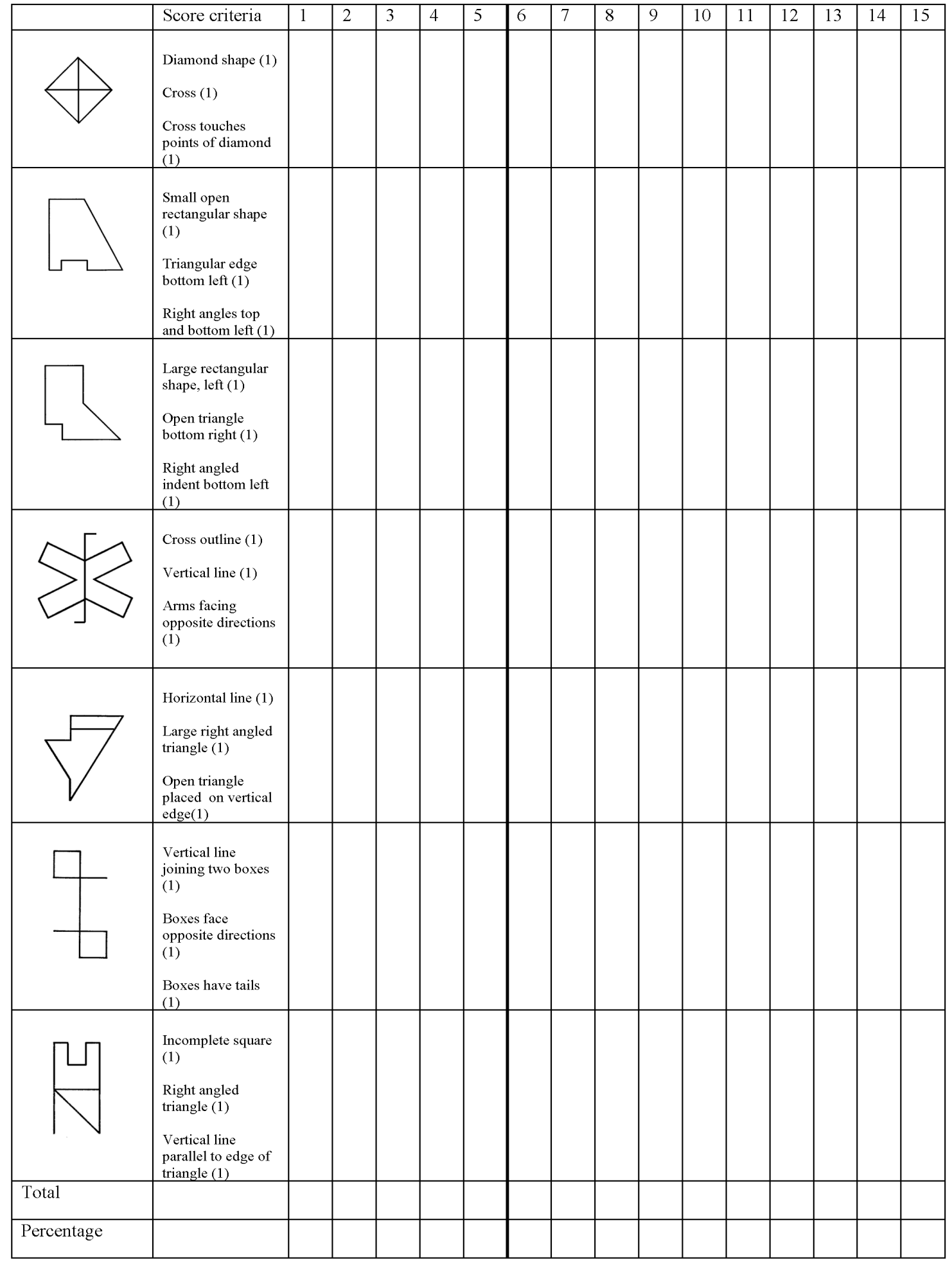


MFD-A EXTENDED RECALL

Criterion $=19 / 21(90 \%)$
Name:

Date:

Start time :

End time:

\begin{tabular}{|c|c|c|c|c|}
\hline & Score criteria & $30 \mathrm{mins}$ & 1 week & 3 weeks \\
\hline & $\begin{array}{l}\text { Diamond shape (1) } \\
\text { Cross (1) } \\
\text { Cross touches } \\
\text { points of diamond } \\
\text { (1) }\end{array}$ & & & \\
\hline & $\begin{array}{l}\text { Small open } \\
\text { rectangular shape } \\
\text { (1) } \\
\text { Triangular edge } \\
\text { bottom left (1) } \\
\text { Right angles top } \\
\text { and bottom left (1) }\end{array}$ & & & \\
\hline & $\begin{array}{l}\text { Large rectangular } \\
\text { shape, left (1) } \\
\text { Open triangle } \\
\text { bottom right (1) } \\
\text { Right angled } \\
\text { indent bottom left } \\
\text { (1) }\end{array}$ & & & \\
\hline & $\begin{array}{l}\text { Cross outline (1) } \\
\text { Vertical line (1) } \\
\text { Arms facing } \\
\text { opposite directions } \\
\text { (1) }\end{array}$ & & & \\
\hline & $\begin{array}{l}\text { Horizontal line (1) } \\
\text { Large right angled } \\
\text { triangle (1) } \\
\text { Open triangle } \\
\text { placed on vertical } \\
\text { edge(1) }\end{array}$ & & & \\
\hline & $\begin{array}{l}\text { Vertical line } \\
\text { joining two boxes } \\
\text { (1) } \\
\text { Boxes face } \\
\text { opposite directions } \\
\text { (1) } \\
\text { Boxes have tails } \\
\text { (1) }\end{array}$ & & & \\
\hline & $\begin{array}{l}\text { Incomplete square } \\
\text { (1) } \\
\text { Right angled } \\
\text { triangle (1) } \\
\text { Vertical line } \\
\text { parallel to edge of } \\
\text { triangle (1) }\end{array}$ & & & \\
\hline Total & & & & \\
\hline Percentage & & & & \\
\hline
\end{tabular}




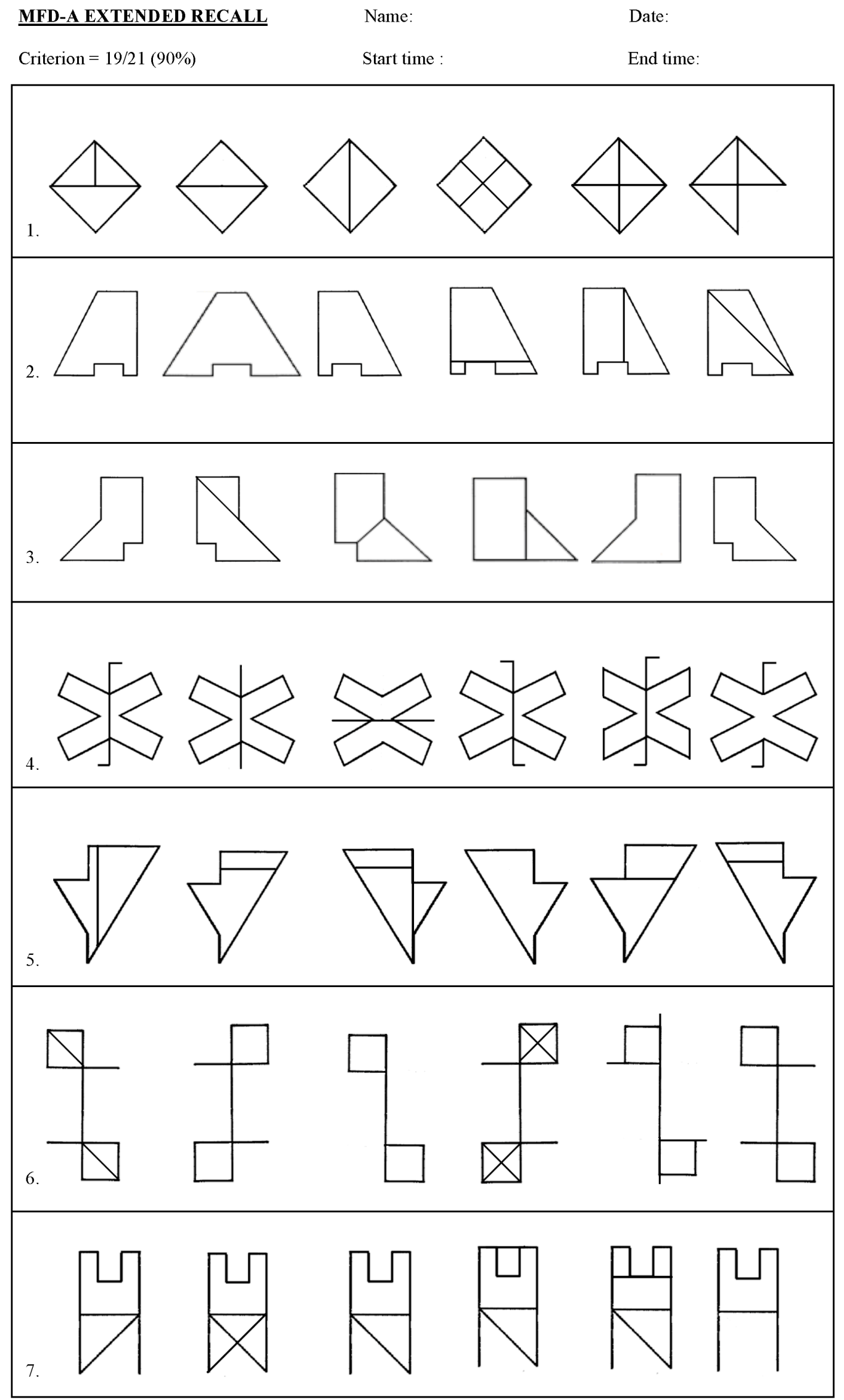


Long-term forgetting task 3: story recall from the Rivermead Behavioural Memory Test (RBMT)

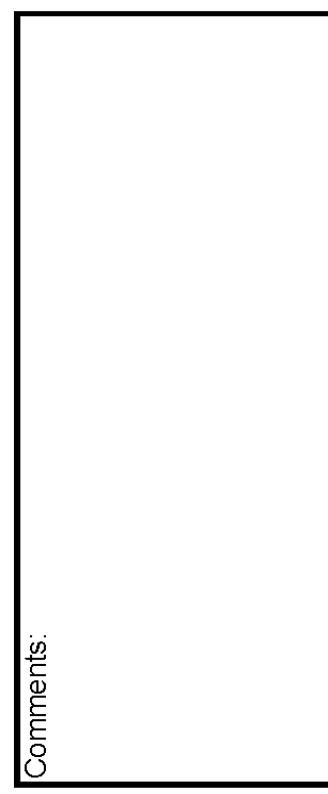

\begin{tabular}{l}
\hline \\
\hline \\
\hline \\
\hline \\
\hline
\end{tabular}

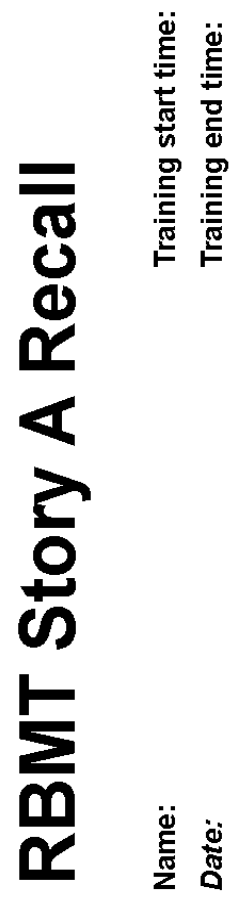

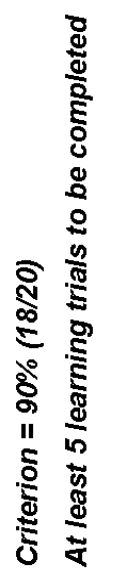

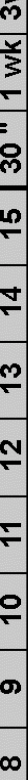

$\wedge$

is

$\nabla$

$\infty$

N

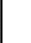

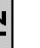

$=$

(1)
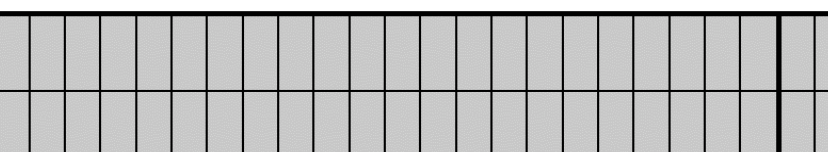

西

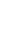

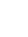

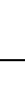

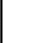

$-$

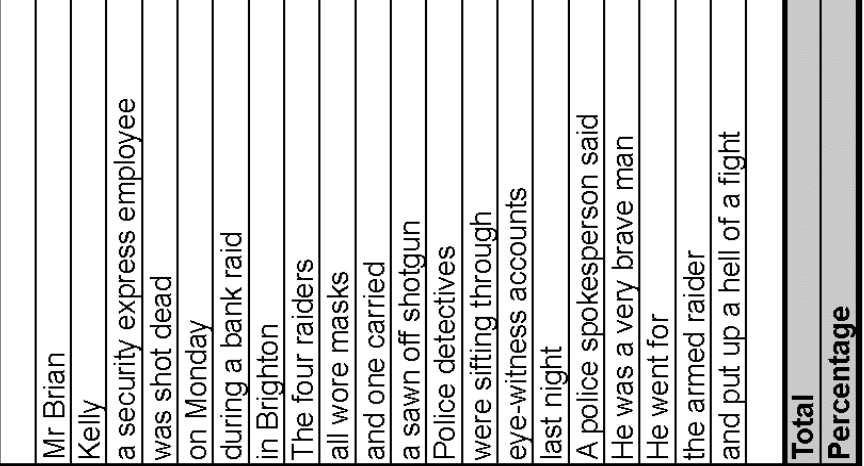




\section{RBMT A Recognition}

\section{To be read aloud to subject}

1. The story was about a:

hijack

mugging

terrorist attack

armed raid

accident

2. What was the name of the main character in the story?

Bill Keely

Brian Jones Bill Kelly

Brian Kelly

Martin Taylor

3. Who employed him?

Security Express

Group Four

Express Delivery

National Express

Home Security

4. Events occurred in a:

post office

jeweller's shop

bank

petrol station

DIY store

5. Where was the story set?

Hove

Bromley

Eastbourne

Leeds

Brighton

6. What happened to the man?

he was shot in the leg he was assaulted

he was shot dead

he was arrested

he had an accident

7. When did this event occur?

morning

early afternoon

Monday

8. How many raiders were there?

Two Four

Five

Three

One

9. What were they wearing?

crash helmets

masks

leather jackets

10. One raider was carrying a:

rifle

revolver

baseball bat

knife

11. Who were 'sifting through' evidence?

police detectives police constables

security guards

criminologists

bank staff

12. What sort of evidence were they looking at? criminal records

eye-witness accounts

forensics

financial accounts

video footage

13. The police described the man as

very tough

very scared

very foolish

very brave

very relieved

14. This was because he: rang the police

15. The man put up

a hell of a fight

a barricade

with tough conditions

a good defence

a battle 
Appendix H 


\section{APPENDIX H}

\section{Modified Autobiographical Memory Interview (MAMI)}

\section{Part I}

\section{First school (5-11 years)}

1. What was the name of your first school?

2. What was your address whilst at this school $($ Road/town only $=1)$ ?

3. Name two teachers or friends from this school

Can you recall any incident, even if small, that occurred to you at this school?

\section{Main secondary school (11-18 years)}

1. What was the name of your main secondary school?

2. How many examinations did you pass and at what level (eg ' $O$ ' or ' $A$ ' level)?

3. Name three teachers or friends from this school

Can you recall any incident, even if small, that occurred to you at this school?

\section{Part II}

Give one question from section A and one from section B for each decade of the subject's life starting at 20 years old.

\section{$\underline{\operatorname{Section}(\mathrm{A})}$}

\section{Career}

Did you change/get promoted in your job/career in your $* *$ s?

(If the answer is confirmed as no move to next topic) 
1. What was the name of the company?

2. Where was it based? (Town?)

3. How did you travel to work?

4. Name two co-workers/colleagues

Can you recall any incident, even if small, that occurred in the workplace in your ${ }^{* *}$ s?

\section{Holidays}

Did you go on holiday during your **s?

(If the answer is confirmed as no move to next topic)

1. Choosing your most memorable holiday in you $* * s$, where exactly did you go? ( $1=$ town/city, $1=$ country $)$

2. What month and year did you go there? $(1=$ month, $1=$ year $)$

3. How long did you stay?

Can you recall any incident, even if small, that happened on holiday in your **s?

\section{Weddings}

Did you attend a wedding in your **s?

1. What was the date of the wedding?

2. Where was the service held? $(1=$ town/city, $1=$ other detail $)$

3. What was the name of the bridegroom?

4. Name one guest not immediately related to you

Can you recall any incident, even if small, from the wedding day? 


\section{$\underline{\text { Section (B) }}$}

\section{Car}

Did you change your car in your **s?

(If the answer is confirmed as no move to next topic)

1. What type of car did you have in your **s? $(1=$ make, $1=$ model $)$

2. What colour was it?

3. Where did you obtain it from?

4. Who else could drive your car?

Can you recall one time when you broke down or took it to get repaired/serviced when you were in your $* * s ?$

\section{Births}

Do you recall anybody other than your self/spouse having a child while you were in your **s? (If the answer is confirmed as no move to next topic)

1. Can you name the parents?

2. What was the child's full name? ( $1=$ first name, $1=$ other names)

3. How old was the child when you first met them?

Can you recall your first meeting with the child?

\section{House}

Did you move house in your **s?

(If the answer is confirmed as no move to next topic)

1. What was the address of your new place? $(1=$ street, $1=$ town/city $)$

2. How many bedrooms?

3. What type of house was it?

4. Name one of your neighbours from this time 
Can you recall any incident, even if small, doing decorating/DIY during your ${ }^{*}$ s?

\section{Hobbies/Interests}

1. Name a hobby/interest you had in your **s?

2. How much time per week did you dedicate to your main one?

3. How did you become interested in this?

4. Name two other people who also have this interest

Can you recall any incident related to your hobby, even if small, that occurred in your **s?

\section{Scoring the episodic autobiographical memories}

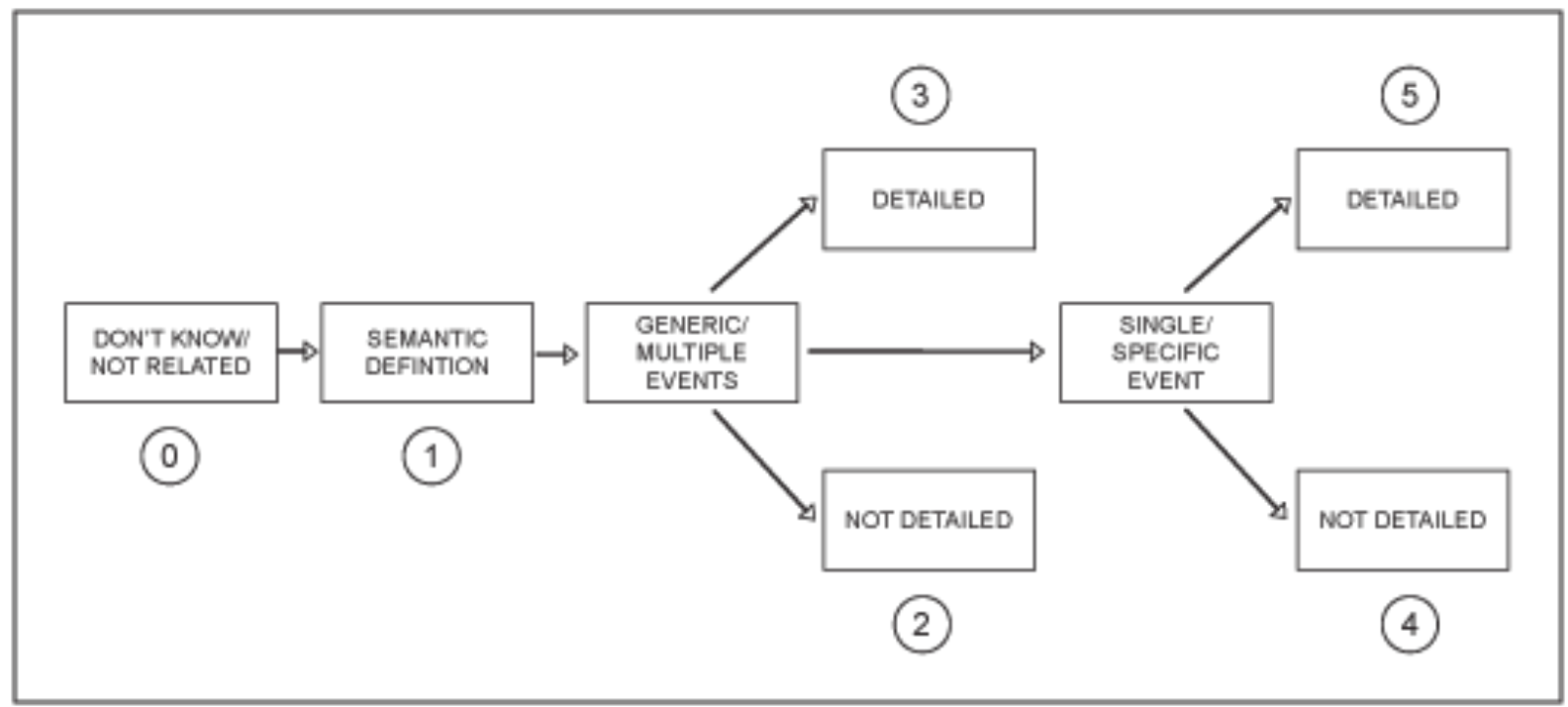




\section{APPENDIX I}

\section{TIME MRI Scanning Protocol}

NAME:

DATE:

STUDY : TIME
CN:

TIME OUT:

IMAGING TIME:
TIME IN:

EXAMINATION NUMBER:

\begin{tabular}{|c|c|c|c|c|c|c|}
\hline COIL & HEAD & & & & & \\
\hline \multicolumn{7}{|l|}{ SEQ. NUMBER } \\
\hline SEQUENCE & T1 SE & $\begin{array}{c}\text { Fast GRE } \\
\text { 3DIR PREP }\end{array}$ & T2 FSE & FLAIR & T2 GRE & DWI \\
\hline $\begin{array}{l}\text { ORIENTATION/ } \\
\text { ANGLE }\end{array}$ & Sagittal & $\begin{array}{l}\text { Coronal } \\
\text { Angle } 90^{\circ} \\
\text { to } \mathrm{AC} / \mathrm{PC} \\
\text { line }\end{array}$ & $\begin{array}{l}\text { Axial } \\
\text { Angle } \\
\text { parallel to } \\
\text { AC/PC line }\end{array}$ & $\begin{array}{l}\text { Axial } \\
\text { Angle } \\
\text { parallel to } \\
\text { AC/PC } \\
\text { line }\end{array}$ & $\begin{array}{l}\text { Axial } \\
\text { Angle } \\
\text { parallel to } \\
\text { AC/PC line }\end{array}$ & $\begin{array}{l}\text { Axial } \\
\text { Angle } \\
\text { parallel } \\
\text { to } \\
\text { AC/PC } \\
\text { line }\end{array}$ \\
\hline TE & MIN & MIN & 102 & 140 & 15 & MIN \\
\hline TR & 450 & & 6300 & 9000 & 625 & 10000 \\
\hline TI & & & & 2200 & & \\
\hline PREP TIME & & 600 & & & & \\
\hline FLIP ANGLE & & 15 & & & 20 & \\
\hline SHOTS & & & & & & 1 \\
\hline b VALUE & & & & & & $\begin{array}{l}1000 \\
\text { (all } \\
\text { directions) }\end{array}$ \\
\hline ETL & & & 24 & & & \\
\hline BAND WIDTH & 15.63 & 31.25 & 15.63 & 15.63 & 12.50 & \\
\hline FOV & 24 & 22 & 24 & 24 & 24 & 24 \\
\hline SLICE THICK. & 5 & 1.7 & 5 & 5 & 5 & 5 \\
\hline SLICE GAP & 1.5 & 0 & 0 & 0 & 0 & 0 \\
\hline MATRIX & $256 \times 224$ & $256 \times 192$ & $256 \times 256$ & $256 \times 224$ & $256 \times 192$ & $128 \times 128$ \\
\hline NEX & 2 & 1 & 2 & 1 & 2 & 1 \\
\hline PHASE FOV & 1 & 1 & 0.75 & & 0.75 & 1 \\
\hline FREQ. DIR & $\mathrm{S} / \mathrm{I}$ & $\mathrm{S} / \mathrm{I}$ & $\mathrm{A} / \mathrm{P}$ & $\mathrm{A} / \mathrm{P}$ & $\mathrm{A} / \mathrm{P}$ & $\mathrm{R} / \mathrm{L}$ \\
\hline NO. SLICES & 19 & 128 & & & & \\
\hline \multicolumn{7}{|l|}{ SAT BANDS } \\
\hline OPTIONS & None & IrP, Fast & $\begin{array}{l}\text { FC,VBW, } \\
\text { FAST, } \\
\text { TRF,Z512 }\end{array}$ & $\begin{array}{l}\text { FC,VBW, } \\
\text { TRF,Fast }\end{array}$ & $\begin{array}{l}\mathrm{FC}, \mathrm{VbW}, \\
\text { EDR }\end{array}$ & $\begin{array}{l}\text { EPI, } \\
\text { DIFF }\end{array}$ \\
\hline
\end{tabular}




\section{Publications, presentations and prizes resulting from the work presented in this thesis}

\section{Scientific Papers}

Butler CR, Bhaduri A, Acosta-Cabronero J, Nestor PJ, Kapur N, Graham KS, Hodges JR, Zeman AZ. Transient epileptic amnesia: regional brain atrophy and its relationship to memory deficits. Brain 2009; 132: 357-68

Butler CR, Zeman AZ. Recent insights into the impairment of memory in epilepsy. Brain 2008; 131: 2243-63.

Butler CR, Zeman AZ. A case of transient epileptic amnesia with radiological localisation. Nat Clin Prac Neurol 2008; 4: 516-21.

Butler CR, Graham KS, Hodges JR, Kapur N, Wardlaw JM, Zeman AZ. The syndrome of transient epileptic amnesia. Ann Neurol, 2007; 61(6): 587-98

Butler, CR. Transient epileptic amnesia. Practical Neurology 2006; 6: 368-71

Butler, CR, Zeman, AZ. Transient amnesic syndromes. Advances in Clinical Neuroscience and Rehabilitation 2006; 6(4): 13-14

\section{Book chapter}

Butler, CR, Zeman AZ. Syndromes of transient amnesia. In Laureys, S, Tonini, G, eds. The Neurology of Consciousness. Elsevier, San Diego (in press) 


\section{Scientific platform presentations}

Butler CR, Bhaduri A, Acosta-Cabronero J, Nestor PJ, Kapur N, Graham KS, Hodges JR, Zeman AZ. Transient epileptic amnesia: regional brain atrophy and its relationship to memory deficits. Association of British Neurologists scientific meeting Sept 2008

Butler CR, Graham KS, Hodges JR, Kapur N, Wardlaw JM, Zeman AZ. Transient epileptic amnesia. British Neuropsychiatry Association annual meeting Mar 2007.

Butler CR, Graham KS, Hodges JR, Kapur N, Wardlaw JM, Zeman AZ. The syndrome of transient epileptic amnesia. Association of British Neurologists scientific meeting Aug 2006.

\section{Invited lectures}

Transient epileptic amnesic: implications for clinical practice and cognitive neuroscience.

Edinburgh Neuroscience Day 2007

The syndrome of transient epileptic amnesia. Memory Disorders Research Society Toronto 2006

\section{Prizes}

Charles Symonds Prize, Association of British Neurologists, Aviemore 2008

Epilepsy Action, Research Prize 2007

Best Platform Presentation, British Neuropsychiatry Association, London 2007

Charles Symonds Prize, Association of British Neurologists, London 2006 